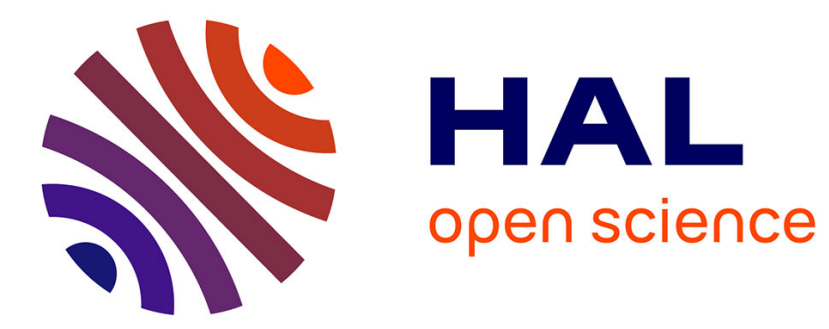

\title{
CONCENTRATION ET RANDOMISATION UNIVERSELLE DE SOUS-ESPACES PROPRES
}

Rafik Imekraz

\section{To cite this version:}

Rafik Imekraz. CONCENTRATION ET RANDOMISATION UNIVERSELLE DE SOUS-ESPACES PROPRES. Analysis \& PDE, 2018, 11 (2), pp.263-350. hal-01564960

\section{HAL Id: hal-01564960 \\ https://hal.science/hal-01564960}

Submitted on 24 Jul 2017

HAL is a multi-disciplinary open access archive for the deposit and dissemination of scientific research documents, whether they are published or not. The documents may come from teaching and research institutions in France or abroad, or from public or private research centers.
L'archive ouverte pluridisciplinaire HAL, est destinée au dépôt et à la diffusion de documents scientifiques de niveau recherche, publiés ou non, émanant des établissements d'enseignement et de recherche français ou étrangers, des laboratoires publics ou privés. 


\title{
CONCENTRATION ET RANDOMISATION UNIVERSELLE DE SOUS-ESPACES PROPRES
}

\author{
par
}

Rafik Imekraz

\begin{abstract}
Résumé. - Nous étudions des conditions nécessaires et suffisantes de convergence pour des séries aléatoires de fonctions propres dans $L^{p}$, avec $p$ fini. De façon précise, nous montrons des résultats optimaux pour les harmoniques sphériques sur $\mathbb{S}^{d}$ et l'oscillateur harmonique sur $\mathbb{R}^{d}$ (cela améliore des résultats de Ayache-Tzvetkov, Grivaux et Imekraz-Robert-Thomann). Dans le cas multidimensionnel, nous utiliserons des séries aléatoires faisant intervenir des matrices aléatoires. Cela nous permettra de donner un éclairage sur la construction d'une famille de mesures construites par Burq-Lebeau sur l'espace de Hilbert d'une variété riemannienne compacte. En fait, nous montrons que c'est précisément parce que $L^{p}$ est de cotype fini que cette construction est possible (il s'agit d'une version multidimensionnelle du théorème de Maurey-Pisier).

Abstract. - We study necessary and sufficient conditions of convergence for random series of eigenfunctions in $L^{p}$, for finite $p$. More precisely, we get optimal results for the spherical harmonics on $\mathbb{S}^{d}$ and for the harmonic oscillator on $\mathbb{R}^{d}$ (this improves results by Ayache-Tzvetkov, Grivaux and Imekraz-Robert-Thomann). In the multidimensional framework, our random series involve random matrices. This enlightens a construction, made by Burq-Lebeau, of a family of specific measures on the Hilbert space of a Riemanniann boundaryless compact manifold. Actually, we show that the latter construction is possible because $L^{p}$ has finite cotype (this is nothing else than a multidimensional version of the Maurey-Pisier theorem).
\end{abstract}

Classification mathématique par sujets (2000). — 60G50, 15B52, 46B09.

Mots clefs. - Randomisation, fonctions propres, matrices aléatoires, injections de Sobolev. 
Table des matières

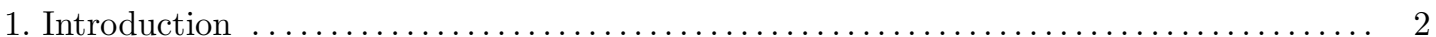

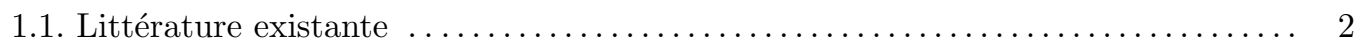

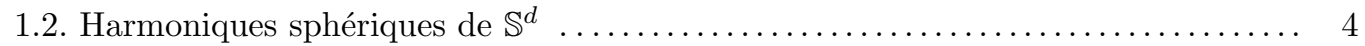

1.3. Oscillateur harmonique $-\Delta+|x|^{2}$ sur $\mathbb{R}^{d} \ldots \ldots \ldots \ldots \ldots \ldots \ldots \ldots \ldots \ldots \ldots$

1.4. Version multidimensionnelle $\mathrm{du}$ théorème de Maurey-Pisier $\ldots \ldots \ldots \ldots \ldots \ldots .9$

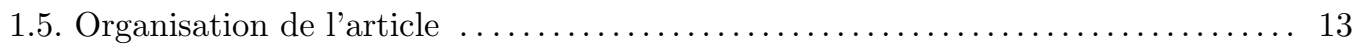

2. Espaces de Lebesgue probabilistes $\ldots \ldots \ldots \ldots \ldots \ldots \ldots \ldots \ldots \ldots \ldots \ldots \ldots \ldots \ldots \ldots$

2.1. Universalité de la randomisation multidimensionnelle dans $L^{p} \ldots \ldots \ldots \ldots \ldots \ldots 14$

2.2. Interpolation et dualité des espaces de Lebesgue probabilistes $\mathbf{P L}^{p} \ldots \ldots \ldots \ldots 16$

2.3. Preuve du théorème 2.1 , randomisation avec des matrices aléatoires $\ldots \ldots \ldots \ldots 18$

2.4. Randomisation dans un treillis de Banach de cotype fini et preuve du théorème

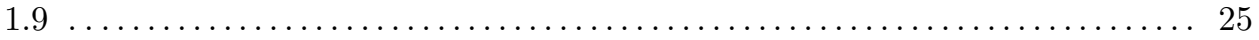

2.5. Preuves des théorèmes 2.5 et 2.6, partie I : rétracte d'un espace de Banach . . . 28

2.6. Preuves des théorèmes 2.5 et 2.6, partie II : espaces de Lorentz . . . . . . . . . . 34

2.7. Preuves des théorèmes 2.5 et 2.6 , partie III : $R$-bornitude $\ldots \ldots \ldots \ldots \ldots \ldots \ldots 35$

2.8. Preuve du théorème 2.6 : bornitude de $S_{p} R_{p} \ldots \ldots \ldots \ldots \ldots \ldots \ldots \ldots \ldots \ldots$

2.9. Preuve du théorème 2.5 : défaut d'interpolation $\ldots \ldots \ldots \ldots \ldots \ldots \ldots \ldots \ldots \ldots 40$

2.10. Preuve du théorème 2.5 : bornitude de $S_{p} R_{p, \psi} \ldots \ldots \ldots \ldots \ldots \ldots \ldots \ldots \ldots 43$

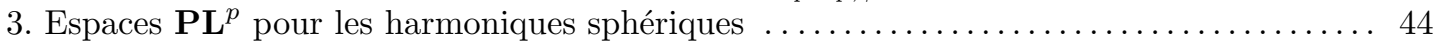

3.1. Reformulation des énoncés $\ldots \ldots \ldots \ldots \ldots \ldots \ldots \ldots \ldots \ldots \ldots \ldots \ldots \ldots \ldots . \ldots \ldots 4$

3.2. Preuve de la proposition 3.2, randomisation des fonctions $Y_{n} \ldots \ldots \ldots \ldots 45$

3.3. Preuve du corollaire 3.3, injections de Sobolev probabilistes des fonctions $Y_{n} \ldots 49$

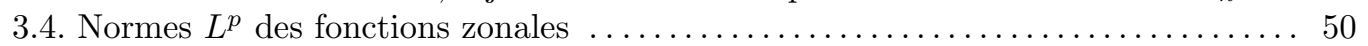

3.5. Preuve de la proposition 3.1, randomisation des fonctions zonales $Z_{n} \ldots \ldots$. . 50

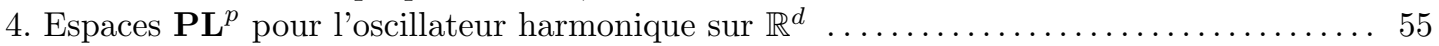

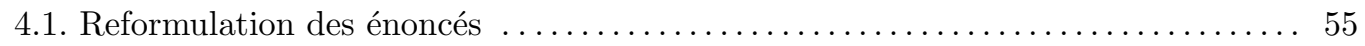

4.2. Rappels sur les fonctions de Hermite $\ldots \ldots \ldots \ldots \ldots \ldots \ldots \ldots \ldots \ldots \ldots \ldots \ldots \ldots . \ldots 9$

4.3. Preuve de la proposition 4.1, majoration (104) de la fonction $e_{d, n} \ldots \ldots \ldots \ldots 60$

4.4. Preuve de la proposition 4.1, minoration (105) de la fonction $e_{d, n} \ldots \ldots \ldots \ldots 61$

4.5. Preuve du théorème 4.3, randomisation des fonctions de Hermite . . . . . . . . 63

4.6. Preuve du théorème 4.4, injections de Sobolev probabilistes hermitiennes . . . . 64

Appendice A. Optimalité de l'exposant $\max (2, p)$ dans le théorème $2.1 \ldots \ldots \ldots \ldots \ldots$

Appendice B. Preuve de la proposition 1.10, inégalité de Latała précisée $(26) \ldots \ldots 6$

Appendice C. Preuve de la proposition 1.10, minoration de la plus petite valeur singulière

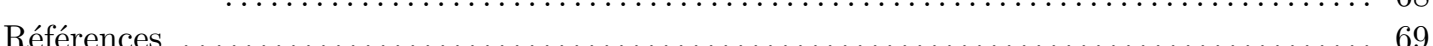

\section{Introduction}

1.1. Littérature existante. — Cet article étudie de manière unifiée les questions suivantes :

i) fixons $p \in[1,+\infty$ [, peut-on trouver des conditions déterministes (nécessaires et suffisantes) qui assurent la convergence dans $L^{p}\left(\mathbb{S}^{d}\right)$ des séries aléatoires constituées par des harmoniques sphériques ?

ii) qu'en est-il pour pour l'oscillateur harmonique $-\Delta+|x|^{2}$ sur la variété non compacte $X=\mathbb{R}^{d}$ ?

iii) quelles séries aléatoires permettent de résoudre les questions précédentes ?

Dans le cas où les fonctions propres considérées ont des propriétés de concentration, nous nous proposons de démontrer des résultats optimaux qui complètent ceux obtenus séparément par Ayache-Tzvetkov [1], Grivaux [27] ainsi qu'un résultat obtenu conjointement par Robert, Thomann et l'auteur dans [30]. En ce qui concerne la randomisation multidimensionnelle, nous obtenons une extension de deux résultats de Maurey et Maurey-Pisier. Cela donnera un éclairage sur la construction d'une famille de mesures sur $L^{2}(X)$ (où $X$ est une variété riemannienne compacte sans bord) construites par Burq-Lebeau [9]. 
Avant d'écrire des énoncés précis, il convient de citer les principaux travaux existants concernant cette problématique. La source de toute cette étude réside dans le théorème de Paley-Zygmund $[\mathbf{4 4 , ~ 4 5 ] ~ : ~ s i ~ l ' o n ~}$ considère une suite $\left(a_{n}\right)_{n \in \mathbb{Z}}$ appartenant à $\ell^{2}(\mathbb{Z})$ et une suite $\left(\varepsilon_{n}\right)_{n \in \mathbb{Z}}$ de variables aléatoires indépendantes suivant une loi $\frac{1}{2}$-Bernoulli à valeurs dans $\{-1,+1\}$ alors la série de Fourier aléatoire $\sum_{n \in \mathbb{Z}} \varepsilon_{n} a_{n} e^{i n x}$ définit presque sûrement un élément de $L^{p}(\mathbb{T})$ pour tout réel $p \in[2,+\infty[$. La randomisation permet ainsi un gain d'intégrabilité alors qu'il n'y a évidemment aucun gain de régularité dans l'échelle des espaces de Sobolev $H^{s}(\mathbb{T})$. Le théorème de Paley-Zygmund peut être considéré comme une amélioration probabiliste de l'injection de Sobolev $H^{\frac{1}{2}-\frac{1}{p}}(\mathbb{T}) \subset L^{p}(\mathbb{T})$ valide pour tout réel $p \in[2,+\infty[$. Les démonstrations du théorème de Paley-Zygmund utilisent généralement l'inégalité de Khintchine. L'ouvrage [32] de Kahane contient de nombreux résultats importants dans ce thème et introduit notamment des versions banachiques de l'inégalité de Khintchine, à savoir les inégalités de Kahane-Khintchine (voir plus loin (39)). Citons maintenant trois résultats connus concernant la randomisation dans les espaces de Lebesgue.

Le premier résultat est le théorème de Maurey et énonce ceci : pour toute suite $\left(u_{n}\right)_{n \in \mathbb{N}}$ de $L^{p}(X)$ où $X$ est un espace mesuré $\sigma$-fini, on a l'équivalence

$$
\sum \varepsilon_{n} u_{n} \quad \text { converge presque sûrement dans } L^{p}(X) \Leftrightarrow \sqrt{\sum_{n \in \mathbb{N}}\left|u_{n}\right|^{2}} \in L^{p}(X) .
$$

En fait, le théorème de Maurey permet de remplacer $L^{p}(X)$ par n’importe quel treillis de Banach qui dispose de la propriété de cotype fini ([40, pages 21-22] et [37, Theorem 1.d.6, Corollary 1.f.9]).

Le deuxième résultat est le théorème de Maurey-Pisier [41, corollaire 1.3] (voir le théorème 1.6 ci-dessous). Ce dernier assure, avec les mêmes notations, que les convergences des séries aléatoires $\sum \varepsilon_{n} u_{n}$ et $\sum g_{n} u_{n}$ dans $L^{p}(X)$ sont équivalentes où $\left(g_{n}\right)_{n \in \mathbb{N}}$ désigne une suite i.i.d. de gaussiennes suivant une loi $\mathcal{N}_{\mathbb{C}}(0,1)$. En fait, le théorème de Maurey-Pisier permet de remplacer $L^{p}(X)$ par un espace de Banach complexe de cotype fini et les gaussiennes $g_{n}$ par des variables aléatoires centrées plus générales.

Le troisième résultat a été obtenu par Figà-Talamanca et Rider qui ont pu remplacer le tore $\mathbb{T}$ par un groupe compact quelconque $G$ dans le théorème de Paley-Zygmund ([23, Theorem 4] et $[\mathbf{2 4}$, Corollary 4]). On pourra aussi consulter [22]. Dans [39], Marcus et Pisier ont résolu le problème de la convergence presque sûre des séries de Fourier dans $L^{\infty}(G)$ et leur analyse permet de retrouver les résultats dans l'échelle des espaces $L^{p}(G)$ avec $p \in[1,+\infty$ [ à l'aide d'une version multidimensionnelle des inégalités de Kahane-Khintchine. Ces dernières inégalités, que nous choisissons de nommer les inégalités de Kahane-Khintchine-Marcus-Pisier (voir plus loin (40)), vont jouer un rôle important dans notre article.

Récemment, ces problèmes ont ressurgi en considérant les fonctions trigonométriques $x \mapsto e^{\text {inx }}$ non pas comme les caractères du groupe abélien compact $\mathbb{T}$ mais plutôt comme les modes propres de l'opérateur de Laplace-Beltrami $\frac{d^{2}}{d x^{2}}$. Deux types de résultat ont notamment motivé cette résurgence :

i) les bases hilbertiennes aléatoires de modes propres ont des propriétés non triviales comme l'ergodicité quantique $[\mathbf{6 3}, \mathbf{4 8}]$ ou des estimations de normes $L^{p}$ bien meilleures que celles des bases hilbertiennes "canoniques" $[4 \mathbf{4 7}, \mathbf{9}]$.

ii) l'étude des équations non-linéaires de type Schrödinger ou ondes sur une variété riemannienne compacte $X$ avec des conditions initiales à faible régularité dans les espaces de Sobolev $H^{s}(X)$ (on parle de régime sur-critique) est un problème difficile en toute dimension et tout spécialement en dimension $\operatorname{dim}(X) \geq 3$. La randomisation donne un gain d'intégrabilité qui permet de construire des solutions qui sont hors d'atteinte avec les méthodes déterministes actuelles. Ces travaux concernent les constructions de mesures de Gibbs (voir les articles $[\mathbf{3}, \mathbf{4}, \mathbf{5}, \mathbf{6 ,} \mathbf{7}, \mathbf{1 0}, \mathbf{1 9}, \mathbf{5 8}]$ et leurs références) ou des conditions initiales aléatoires plus générales $[\mathbf{1 1}, \mathbf{1 2}, \mathbf{1 3}, \mathbf{1 4}, \mathbf{4 6}]$. Concernant ces derniers articles, on pourra consulter le séminaire Bourbaki $[\mathbf{1 7}]$.

Les deux points précédents ont même été combinés dans [18] : de Suzzoni a étudié l'équation cubique des ondes sur la sphère $\mathbb{S}^{3}$ à l'aide d'une base hilbertienne aléatoire de $L^{2}\left(\mathbb{S}^{3}\right)$.

Il est donc légitime d'étudier la randomisation non plus sur un groupe compact mais sur une variété riemannienne compacte $X$ (que l'on supposera toujours lisse, sans bord et de dimension $d \geq 2$ ). Étant données une suite $\left(a_{n}\right) \in \ell^{2}(\mathbb{N})$, une famille orthonormée $\left(\phi_{n}\right)_{n \geq 0}$ de $L^{2}(X)$ constituée de fonctions propres de l'opérateur de Laplace-Beltrami $\Delta$ sur $X$ et la fonction

$$
\sum_{n \geq 0} a_{n} \phi_{n} \in L^{2}(X)
$$


on s'intéresse à la convergence presque sûre dans $L^{p}(X)$ de la série aléatoire $\sum \varepsilon_{n} a_{n} \phi_{n}$. Bien entendu, le critère (1) répond à la question de façon théorique mais il ne paraît pas évident de le traduire en un comportement asymptotique sur la suite des coefficients $\left(a_{n}\right)_{n \in \mathbb{N}}$. Sans surprise, cette étude est intimement liée à la suite des normes $\left\|\phi_{n}\right\|_{L^{p}(X)}$ en vertu de l'inégalité triangulaire :

$$
\forall p \in\left[2,+\infty\left[\quad\left\|\sqrt{\sum_{n \in \mathbb{N}}\left|a_{n}\right|^{2}\left|\phi_{n}\right|^{2}}\right\|_{L^{p}(X)} \leq\left(\sum_{n \in \mathbb{N}}\left|a_{n}\right|^{2}\left\|\phi_{n}\right\|_{L^{p}(X)}^{2}\right)^{1 / 2} .\right.\right.
$$

En fait, nous verrons que c'est l'éventuelle concentration des fonctions $\phi_{n}$ qui rentre en jeu. Rappelons les résultats connus. Tzvetkov montre ce que l'on appellera par la suite une injection de Sobolev probabiliste (selon la terminologie introduite par Burq et Lebeau dans [9]) : pour tout réel $p \in] 2,+\infty[$ il existe un réel explicite $\delta(d, p)<d\left(\frac{1}{2}-\frac{1}{p}\right)$ tel que

$$
\sum_{n \geq 0} a_{n} \phi_{n} \in H^{\delta(d, p)}(X) \Rightarrow \sum_{n \geq 0} \varepsilon_{n} a_{n} \phi_{n} \quad \text { converge p.s. dans } L^{p}(X) \text {. }
$$

Avant de donner l'expression de $\delta(d, p)$, signalons que (3) améliore l'injection de Sobolev déterministe

$$
\sum_{n \geq 0} a_{n} \phi_{n} \in H^{d\left(\frac{1}{2}-\frac{1}{p}\right)}(X) \Rightarrow \sum_{n \geq 0} a_{n} \phi_{n} \in L^{p}(X) .
$$

Par construction (voir [59, Theorem 4]), le nombre $\delta(d, p)$ vient des inégalités de Sogge [51] que nous rappelons : si l'on a $\Delta \phi_{n}=-\lambda_{n}^{2} \phi_{n}$, avec $\lambda_{n}>0$ alors on a

$$
\left\|\phi_{n}\right\|_{L^{p}(X)} \leq C(X, p) \lambda_{n}^{\delta(d, p)}, \quad \delta(d, p):=\left\{\begin{array}{lll}
\frac{d-1}{2}\left(\frac{1}{2}-\frac{1}{p}\right) & \text { si } \quad 2 \leq p \leq \frac{2(d+1)}{d-1} \\
\frac{d-1}{2}-\frac{d}{p} & \text { si } \quad \frac{2(d+1)}{d-1} \leq p \leq+\infty
\end{array}\right.
$$

où $C(X, p) \geq 1$ ne dépend que de $X$ et $p$. Le cas $p=\infty$ est dû à Avakumovič, Levitan et Hörmander [29] et les inégalités (4) sont optimales pour $X=\mathbb{S}^{d}$. Notons au passage que le critère de Maurey (1) et l'inégalité triangulaire (2) permettent de retrouver immédiatement l'implication (3).

De façon indépendante, des travaux font intervenir la notion de randomisation multidimensionnelle sur une variété riemannienne compacte $X$ en tenant compte de la décomposition spectrale de l'opérateur de LaplaceBeltrami sur $L^{2}(X)$. Cette notion apparaît sous des formes en apparence différente, dans les travaux de Shiffman et Zelditch [50, 63], dans celui de Burq et Lebeau [9] ainsi que dans celui de Poiret, Robert et Thomann [47] (pour l'oscillateur harmonique sur $\mathbb{R}^{d}$ ) avec des arguments de "concentration de la mesure" et de "grandes déviations". C'est dans l'article [9] que le terme "injection de Sobolev probabiliste" apparaît pour la première fois pour exprimer rigoureusement le gain d'intégrabilité obtenu par la randomisation. Même si cela n'est pas explicitement écrit, il nous semble qu'un des intérêts du papier [9] est précisément de s'émanciper de l'astuce de considérer des modes propres invariants par symétrie (ce qui permet usuellement de réduire un problème multidimensionnel à un problème unidimensionnel). C'est ainsi que Burq et Lebeau obtiennent un résultat d'existence locale pour l'équation semi-linéaire des ondes sur une variété riemannienne compacte en régime sur-critique et leurs solutions ne sont pas spectralement supportées par des sous-suites particulières de fonctions propres.

Dans notre article, nous étudions les propriétés de dualité et interpolation de nouveaux espaces de Banach associés à une suite de sous-espaces propres $\left(E_{n}\right)_{n \geq 1}$, dénommés plus loin espaces de Lebesgue probabilistes et notés $\mathbf{P} \mathbf{L}^{p}\left(X, \oplus E_{n}\right)$. Afin de motiver cette étude dans le cadre de la randomisation des fonctions propres, nous écrivons dans les trois prochaines parties les applications que nous obtenons.

1.2. Harmoniques sphériques de $\mathbb{S}^{d}$. - Dans [1, Theorem 1], Ayache et Tzvetkov obtiennent un éclairage gaussien du théorème de Paley-Zygmund, à savoir l'équivalence pour tout $p \in[2,+\infty[$ des deux assertions suivantes :

i) les fonctions propres $\phi_{n}$ sont uniformément bornées dans $L^{p}(X)$,

ii) pour toute suite complexe $\left(a_{n}\right)_{n \in \mathbb{N}}$, la fonction gaussienne aléatoire $\sum_{n \geq 0} g_{n} a_{n} \phi_{n}$ appartient presque sûrement à $L^{p}(X)$ si et seulement si $\left(a_{n}\right)_{n \in \mathbb{N}}$ appartient à $\ell^{2}(\mathbb{N})$. 
Alors que les preuves usuelles du théorème de Paley-Zygmund utilisent l'égalité $\left|e^{i n x}\right|=1$, l'équivalence i) $\Leftrightarrow$ ii) montre que c'est plutôt l'inégalité $\sup _{n \in \mathbb{N}}\left\|e^{i n x}\right\|_{L_{x}^{p}(-\pi, \pi)}<+\infty$ qui est déterminante. En d'autres termes, seule l'explosion des normes $\left\|\phi_{n}\right\|_{L^{p}}$ peut contredire la conclusion du théorème de Paley-Zygmund. Dans ce cas, Ayache et Tzvetkov posent la question de calculer en fonction des coefficients $a_{n}$, la borne supérieure des réels $p \in\left[2,+\infty\right.$ [ tels que la série aléatoire $\sum g_{n} a_{n} \phi_{n}$ converge presque sûrement dans $L^{p}(X)$. Sans aucune information sur les fonctions $\phi_{n}$, cette question est trop générale et Ayache et Tzvetkov examinent les modes propres radiaux $\psi_{n}$ de l'opérateur Laplacien $\Delta$ avec condition de Dirichlet au bord sur la boule fermée unité $\overline{\mathbb{B}_{d}(0,1)}$ de $\mathbb{R}^{d}$. Il s'avère que les fonctions $\psi_{n}$ ne sont pas uniformément bornées dans $L^{p}\left(\mathbb{B}_{d}(0,1)\right)$ pour $p \gg 1$, et l'on en déduit l'existence de suites $\left(a_{n}\right)_{n \in \mathbb{N}} \in \ell^{2}(\mathbb{N})$ telles que la série aléatoire gaussienne $\sum g_{n} a_{n} \psi_{n}$ diverge presque sûrement dans $L^{p}\left(\mathbb{B}_{d}(0,1)\right)$. Il s'agit là d'une différence majeure avec le théorème de Paley-Zygmund sur le tore $\mathbb{T}^{d}$. Dans le cas des fonctions $\psi_{n}$, Ayache et Tzvetkov obtiennent une réponse partielle [1, Theorem 4]. Dans [27] apparaissent deux idées qui vont jouer un rôle important dans notre travail :

i) d'une part, Grivaux remarque que le théorème de Maurey-Pisier assure l'équivalence des convergences des séries aléatoires $\sum \varepsilon_{n} a_{n} \psi_{n}$ et $\sum g_{n} a_{n} \psi_{n}$.

ii) d'autre part, Grivaux répond à la question de Ayache et Tzvetkov en utilisant la concentration des fonctions $\psi_{n}$ en l'origine $0 \in \mathbb{R}^{d}$.

Comme le remarquent Ayache et Tzvetkov [1, Theorem 4, remark (d)], l'analyse précédente se transfère sans problème aux fonctions propres zonales de l'opérateur de Laplace-Beltrami sur la sphère

$$
\mathbb{S}^{d}:=\left\{\left(x_{1}, \ldots, x_{d+1}\right) \in \mathbb{R}^{d+1}, \quad x_{1}^{2}+\cdots+x_{d+1}^{2}=1\right\}, \quad d \geq 2 .
$$

On notera $\mu_{d}$ la mesure volume de $\mathbb{S}^{d}$ et l'on rappelle que le spectre de l'opérateur de Laplace-Beltrami $\Delta$ est donné par la suite $(-n(n+d-1))_{n \in \mathbb{N}}$. Convenons aussi qu'une fonction sur $\mathbb{S}^{d}$ est zonale si elle ne dépend que de la première coordonnée $x_{1}$ d'un point $x \in \mathbb{S}^{d}$. D'après $[\mathbf{5 3}$, Chapter IV.2, Theorem 2.14 , page 149], on sait que l'opérateur de Laplace-Beltrami $\Delta$ admet une suite de fonctions propres zonales $\left(Z_{n}\right)_{n \geq 1}$ vérifiant :

$$
Z_{n}(x)=n^{\frac{1}{2}} P_{n}^{\left(\frac{d-2}{2}, \frac{d-2}{2}\right)}\left(x_{1}\right), \quad \Delta Z_{n}=-n(n+d-1) Z_{n}, \quad\left\|Z_{n}\right\|_{L^{2}\left(\mathbb{S}^{d}\right)} \simeq_{d} 1,
$$

où $P_{n}^{\left(\frac{d-2}{2}, \frac{d-2}{2}\right)}$ est le $n$-ième polynôme de Jacobi, c'est-à-dire le $n$-ième polynôme orthogonal pour le poids $w \in[-1,1] \mapsto\left(1-w^{2}\right)^{\frac{d-2}{2}}$ et normalisé de sorte que

$$
\forall n \in \mathbb{N}^{\star} \quad P_{n}^{\left(\frac{d-2}{2}, \frac{d-2}{2}\right)}(1)=\left(\begin{array}{c}
n+\frac{d-2}{2} \\
n
\end{array}\right) \simeq_{d} n^{\frac{d-2}{2}} .
$$

On peut montrer que l'on a les estimations (voir partie 3.4) :

$$
\begin{aligned}
& 1 \leq p<\frac{2 d}{d-1} \quad \Rightarrow \quad\left\|Z_{n}\right\|_{L^{p}\left(\mathbb{S}^{d}\right)} \quad \simeq_{d, p} \quad 1, \\
& p=\frac{2 d}{d-1} \quad \Rightarrow \quad\left\|Z_{n}\right\|_{L^{p}\left(\mathbb{S}^{d}\right)} \quad \simeq_{d, p} \quad \sqrt[p]{\ln (n+1)}, \\
& \frac{2 d}{d-1}<p<\infty \Rightarrow\left\|Z_{n}\right\|_{L^{p}\left(\mathbb{S}^{d}\right)} \simeq_{d, p} n^{\frac{d-1}{2}-\frac{d}{p}} .
\end{aligned}
$$

D'après [1] , on sait que pour toute suite $\left(a_{n}\right) \in \ell^{2}\left(\mathbb{N}^{\star}\right)$ et tout $p \in\left[2, \frac{2 d}{d-1}\left[\right.\right.$, la série aléatoire $\sum g_{n} a_{n} Z_{n}$ converge presque sûrement dans $L^{p}\left(\mathbb{S}^{d}\right)$. Pour comprendre ce qu'il en est pour $p>\frac{2 d}{d-1}$, on pourrait appliquer la méthode de Grivaux [27]. Cette méthode nécessite de rappeler que les fonctions zonales $Z_{n}$ se concentrent autour de deux boules centrées aux pôles $( \pm 1,0, \ldots, 0)$ de rayon d'ordre $\frac{1}{n}$ avec une amplitude d'ordre $n^{\frac{d-1}{2}}$. Remarquons au passage que les formules (6) expliquent que cette concentration polaire est significative dans $L^{p}\left(\mathbb{S}^{d}\right)$ pour $p>\frac{2 d}{d-1}$. Notons maintenant

$$
p_{c}:=\sup \left\{p>\frac{2 d}{d-1}, \quad \frac{1}{n^{d+1}}\left(\sum_{k=1}^{n} k^{d-1}\left|a_{k}\right|^{2}\right)^{\frac{p}{2}}=\mathcal{O}\left(\frac{1}{n}\right)\right\} .
$$

Alors pour tout réel $p \in[2,+\infty$ [, la méthode de Grivaux donne les implications suivantes

$$
\begin{aligned}
& p<p_{c} \Rightarrow \text { la série aléatoire } \sum g_{n} a_{n} Z_{n} \text { converge p.s. dans } L^{p}\left(\mathbb{S}^{d}\right) \\
& p>p_{c} \Rightarrow \text { la série aléatoire } \sum g_{n} a_{n} Z_{n} \text { diverge p.s. dans } L^{p}\left(\mathbb{S}^{d}\right)
\end{aligned}
$$




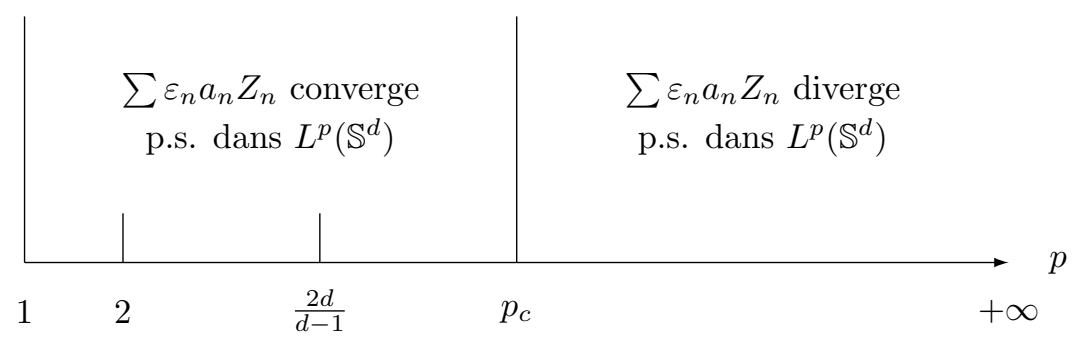

Les arguments connus ne permettent pas de décider ce qu'il en est en $p=p_{c}$ (question soulevée dans [30, remark pages 2776-7] qui utilise notamment la méthode de Grivaux pour les fonctions propres radiales de l'opérateur $\left.-\Delta+|x|^{2}\right)$. Le premier résultat que nous énonçons élucide ce point.

Théorème 1.1. - On considère une suite complexe $\left(a_{n}\right)_{n \geq 1}$ à croissance polynomiale et un réel $p \in$ ]$\frac{2 d}{d-1},+\infty\left[\right.$. La série aléatoire $\sum g_{n} a_{n} Z_{n}$ converge presque sûrement dans $L^{p}\left(\mathbb{S}^{d}\right)$ si et seulement si

$$
\sum_{n \geq 1} \frac{1}{n^{d+1}}\left(\sum_{k=1}^{n} k^{d-1}\left|a_{k}\right|^{2}\right)^{\frac{p}{2}}<+\infty
$$

Le théorème précédent nous permet de préciser la formule (7) par comparaison logarithmique et rend légitime la mise en évidence de son terme $\mathcal{O}\left(\frac{1}{n}\right)$. Examinons par exemple des coefficients de la forme

$$
\alpha_{n}=\frac{1}{n^{d\left(\frac{1}{2}-\frac{1}{p}\right)} \ln ^{\frac{\beta}{p}}(n)}, \quad \beta \geq 0, \quad p>\frac{2 d}{d-1}, \quad n \geq 2 .
$$

La suite $\left(\alpha_{n}\right)_{n \geq 2}$ appartient à $\ell^{2}(\mathbb{N} \backslash\{0,1\})$ et l'on calcule facilement l'équivalent

$$
\frac{1}{n^{d+1}}\left(\sum_{k=2}^{n} k^{d-1}\left|\alpha_{k}\right|^{2}\right)^{\frac{p}{2}} \sim\left(\frac{p}{2 d}\right)^{\frac{p}{2}} \frac{1}{n \ln ^{\beta}(n)}, \quad n \rightarrow+\infty .
$$

La borne supérieure (7) vaut donc $p$ pour toute valeur de $\beta$, mais le théorème 1.1 permet d'affirmer que la série aléatoire $\sum_{n>2} g_{n} \alpha_{n} Z_{n}$ converge presque sûrement dans $L^{p}\left(\mathbb{S}^{d}\right)$ si et seulement si $\beta>1$.

Donnons une idée de la preuve du théorème 1.1. Nous justifierons rigoureusement que l'on peut assimiler $\left|Z_{n}\right|$ à sa restriction $\widetilde{Z}_{n}$ sur une boule de rayon d'ordre $\frac{1}{n}$ et centrée en l'un des pôles de symétrie. Cela nous permettra d'obtenir l'encadrement suivant pour tout $p>\frac{2 d}{d-1}$ :

$$
\left\|\sqrt{\sum_{n \geq 1}\left|a_{n} \widetilde{Z}_{n}\right|^{2}}\right\|_{L^{p}\left(\mathbb{S}^{d}\right)} \leq\left\|\sqrt{\sum_{n \geq 1}\left|a_{n} Z_{n}\right|^{2}}\right\|_{L^{p}\left(\mathbb{S}^{d}\right)} \leq C(d, p)\left\|\sqrt{\sum_{n \geq 1}\left|a_{n} \widetilde{Z}_{n}\right|^{2}}\right\|_{L^{p}\left(\mathbb{S}^{d}\right)},
$$

où $C(d, p) \geq 1$ ne dépend que de $d$ et $p$. Comme les normes dans $L^{\frac{p}{2}}\left(\mathbb{S}^{d}\right)$ des fonctions $\sum_{n \geq 1}\left|a_{n} \widetilde{Z}_{n}\right|^{2}$ sont calculables explicitement, on pourra finir en invoquant le critère (1). La majoration (8) est délicate et utilisera de nouveaux résultats abstraits d'interpolation développés dans la partie 2.2. Signalons dès maintenant que l'interpolation (réelle ou complexe) des normes qui apparaissent dans (8) n'est pas gratuite et découlera des propriétés de concentration des fonctions zonales $Z_{n}$. Par exemple, il découlera de (6) et de la proposition 2.4 que l'interpolation n'est pas réalisée si l'on autorise $p$ à parcourir un intervalle ouvert contenant $\frac{2 d}{d-1}$.

Nos arguments permettent de traiter un autre cas important de fonctions propres, à savoir la suite $\left(Y_{n}\right)_{n \geq 1}$ des fonctions propres "de plus haut poids" qui se concentrent sur une géodésique. Ces dernières sont définies par les formules

$$
\forall n \in \mathbb{N}^{\star} \quad Y_{n}(x):=c_{d, n}\left(x_{1}+i x_{2}\right)^{n}, \quad\left\|Y_{n}\right\|_{L^{2}\left(\mathbb{S}^{d}\right)}=1,
$$

où $c_{d, n}>0$ est une constante de normalisation. On vérifie que l'on a $\Delta Y_{n}=-n(n+d-1) Y_{n}$ et $c_{d, n} \simeq_{d}$ $n^{\frac{d-1}{4}}$. Les fonctions $Y_{n}$ sont connues pour avoir des estimations de normes dans $L^{p}\left(\mathbb{S}^{d}\right)$ maximales pour $2<p \leq \frac{2(d+1)}{d-1}$ et minimales pour $p \in\left[1,2\left[\right.\right.$ parmi les modes propres $L^{2}\left(\mathbb{S}^{d}\right)$-normalisés associés à la valeur 
propre $-n(n+d-1)$ (et cela est même optimal d'après les inégalités de Sogge (4) et [52, Proposition 2]). De manière précise, il est connu et facile à vérifier que (9) implique les estimations suivantes

$$
\forall p \in\left[1,+\infty\left[\cup\{+\infty\} \quad \forall n \in \mathbb{N}^{\star} \quad\left\|Y_{n}\right\|_{L^{p}\left(\mathbb{S}^{d}\right)} \simeq_{d, p} n^{\frac{d-1}{2}\left(\frac{1}{2}-\frac{1}{p}\right)} .\right.\right.
$$

On montrera le théorème suivant.

Théorème 1.2. - On considère une suite complexe $\left(a_{n}\right)_{n \geq 1}$ à croissance polynomiale. Pour tout réel $p \in] 1,+\infty\left[\right.$, la série aléatoire $\sum g_{n} a_{n} Y_{n}$ converge presque sûrement dans $L^{p}\left(\mathbb{S}^{d}\right)$ si et seulement si

$$
\sum_{n \geq 1} \frac{1}{n^{\frac{d+1}{2}}}\left(\sum_{k=1}^{n} k^{\frac{d-1}{2}}\left|a_{k}\right|^{2}\right)^{\frac{p}{2}}<+\infty .
$$

Comme application immédiate, on obtient le fait suivant qui contraste avec le théorème de Paley-Zygmund sur $\mathbb{T}$ : la fonction $\sum_{n \geq 2} \frac{1}{\sqrt{n} \ln (n)} Y_{n}$ appartient à $L^{2}\left(\mathbb{S}^{d}\right)$ mais la série aléatoire $\sum \frac{g_{n}}{\sqrt{n} \ln (n)} Y_{n}$ diverge presque sûrement dans $L^{p}\left(\mathbb{S}^{d}\right)$ pour tout réel $p>2$. La preuve du théorème 1.2 consistera à estimer les normes

$$
\left\|\sqrt{\sum_{n \geq 1}\left|a_{n} Y_{n}\right|^{2}}\right\|_{L^{p}\left(\mathbb{S}^{d}\right)}
$$

Cela sera très facile pour $p \in 2 \mathbb{N}$ en utilisant des estimées optimales vérifiées par les intégrales

$$
\int_{\mathbb{S}^{d}}\left|Y_{n_{(1)}}(x) \ldots Y_{n_{(p / 2)}}(x)\right|^{2} d \mu_{d}(x), \quad\left(n_{(1)}, \ldots, n_{(p / 2)}\right) \in\left(\mathbb{N}^{\star}\right)^{\frac{p}{2}} .
$$

Pour traiter le cas général $p \in] 1,+\infty[$, on raisonnera par interpolation et dualité. Mais comme précédemment, cela n'est pas gratuit. De façon précise, la concentration gaussienne de $Y_{n}$ autour de la géodésique $\left\{x_{1}^{2}+x_{2}^{2}=\right.$ 1\} $\subset \mathbb{S}^{d}$ nous permettra de valider les hypothèses de nos nouveaux théorèmes d'interpolation et de dualité (théorèmes 2.5 et 2.6).

Remarque 1.3. - La condition (11) est exactement celle que l'on obtient avec le critère (1) en remplaçant $Y_{n}$ par la fonction $x \mapsto n^{\frac{d-1}{4}} \mathbf{1}_{V_{n}}(x)$ où $V_{n} \subset \mathbb{S}^{d}$ est une bande de largeur $\frac{1}{\sqrt{n}}$ autour de la géodésique d'équation $x_{1}^{2}+x_{2}^{2}=1$. Par souci de simplification, nous n'avons traité que le cas des géodésiques sur $\mathbb{S}^{d}$ mais notre démarche est en fait plus générale. En effet, on devrait pouvoir estimer les intégrales (12) en utilisant seulement la concentration gaussienne de $Y_{n}$ autour de la géodésique $\left\{x_{1}^{2}+x_{2}^{2}=1\right\}$. Or ce type de concentration se réalise dans un autre cas important, à savoir celui d'une surface $X$ qui admet une géodésique $\Gamma \subset X$ fermée elliptique et non-dégénérée. Il est alors connu, mais assez délicat à rédiger, que l'on peut construire des quasi-modes qui se concentrent autour de la géodésique $\Gamma$ avec des estimations gaussiennes (ce sont les travaux de Ralston et Babich).

En résumé, les théorèmes 1.1 et 1.2 affirment que la concentration sur une zone déterminée (voisinage d'une géodésique ou d'un pôle) est la seule information pertinente pour étudier les séries aléatoires de fonctions propres. Malgré la ressemblance de leurs énoncés, les preuves des théorèmes 1.1 et 1.2 vont être différentes car la concentration des fonctions $Y_{n}$ autour de la géodésique $\left\{x_{1}^{2}+x_{2}^{2}=1\right\} \subset \mathbb{S}^{d}$ est bien meilleure que la concentration des fonctions zonales $Z_{n}$ autour des pôles $( \pm 1,0, \ldots, 0)$ (voir la discussion concernant les estimées multilinéaires au début de la preuve de la proposition 3.1).

Les deux exemples de fonctions propres $Y_{n}$ et $Z_{n}$ nous permettront facilement de justifier l'optimalité de l'injection de Sobolev probabiliste (3) obtenue par Tzvetkov dans [59]. Cela est parfaitement cohérent car les preuves de [59] sont obtenues grâce aux inégalités de Sogge (4) qui sont précisément saturées pour les fonctions $Y_{n}$ et $Z_{n}$.

1.3. Oscillateur harmonique $-\Delta+|x|^{2}$ sur $\mathbb{R}^{d}$. - Dans cette partie, nous expliquons notre principale application à l'oscillateur harmonique $-\Delta+|x|^{2} \operatorname{sur} L^{2}\left(\mathbb{R}^{d}\right)$ avec $d \geq 2$. Nous prolongeons l'étude entamée dans [30]. Pour tout entier $n \in \mathbb{N}$, on note $H_{n} \in \mathbb{R}[X]$ le $n$-ième polynôme de Hermite ainsi que $h_{n}$ la $n$-ième fonction de Hermite :

$$
H_{n}(x):=(-1)^{n} e^{x^{2}} \frac{d^{n}}{d x^{n}} e^{-x^{2}} \quad \text { et } \quad h_{n}(x):=\frac{H_{n}(x)}{\sqrt{n ! 2^{n} \sqrt{\pi}}} e^{-\frac{1}{2} x^{2}} .
$$


On vérifie que l'on a $\left\|h_{n}\right\|_{L^{2}(\mathbb{R})}=1$. On définit aussi le sous-espace suivant de $L^{2}\left(\mathbb{R}^{d}\right)$

$$
E_{d, n}:=\operatorname{Vect}\left\{h_{i_{1}} \otimes \cdots \otimes h_{i_{d}} ; \quad\left(i_{1}, \ldots, i_{d}\right) \in \mathbb{N}^{d}, \quad i_{1}+\cdots+i_{d}=n\right\},
$$

où l'on note classiquement $\left(h_{i_{1}} \otimes \cdots \otimes h_{i_{d}}\right)(x)=h_{i_{1}}\left(x_{1}\right) \ldots h_{i_{d}}\left(x_{d}\right)$ pour tout $x \in \mathbb{R}^{d}$. Les fonctions $h_{i_{1}} \otimes \cdots \otimes h_{i_{d}}$ forment une base hilbertienne de $E_{d, n}$ et l'on vérifie aussi que l'on a

$$
d_{n}:=\operatorname{dim}\left(E_{d, n}\right)=\frac{(n+1) \ldots(n+d-1)}{(d-1) !} \sim \frac{n^{d-1}}{(d-1) !}, \quad n \rightarrow+\infty .
$$

Il faut savoir de plus que $E_{d, n}$ est le sous-espace propre de $-\Delta+|x|^{2}$ associé à la valeur propre $d+2 n$ et que l'on a la somme directe orthogonale

$$
L^{2}\left(\mathbb{R}^{d}\right)=\bigoplus_{n \in \mathbb{N}} E_{d, n}
$$

On veut associer à cette somme directe orthogonale des séries aléatoires et étudier leur convergence en probabilité dans $L^{p}\left(\mathbb{R}^{d}\right)$. Expliquons d'abord la démarche employée dans [30, line (1.9)]. Les séries aléatoires précédemment utilisées sont de la forme

$$
\omega \in \Omega \mapsto \sum_{n \in \mathbb{N}} \sum_{k=1}^{d_{n}} V_{n, k}(\omega) c_{n, k} \phi_{n, k},
$$

où $\Omega$ est un univers probabilisé de référence, $\left(\phi_{n, k}\right)_{1 \leq k \leq d_{n}}$ est une base hilbertienne de $E_{d, n},\left(V_{n, k}\right)_{n, k}$ est une famille de variables aléatoires, i.i.d., centrées, non nulles et dont tous les moments sont finis, et $\left(c_{n, k}\right)_{n, k}$ est une suite de coefficients complexes. D'après le critère de Maurey (1) et le théorème de Maurey-Pisier, la convergence de ces séries aléatoires dans $L^{p}\left(\mathbb{R}^{d}\right)$ revient à étudier les normes

$$
\left\|\sum_{n \in \mathbb{N}} \sum_{k=1}^{d_{n}}\left|c_{n, k}\right|^{2}\left|\phi_{n, k}(x)\right|^{2}\right\|_{L_{x}^{p / 2}\left(\mathbb{R}^{d}\right)} .
$$

L'expression précédente dépend a priori de chaque base hilbertienne $\left(\phi_{n, k}\right)$ de $E_{d, n}$. Afin d'obtenir des résultats indépendants de ces bases, une condition de contrôle (nommée squeezing condition) sur les coefficients $\left(c_{n, k}\right)$ a été imposée dans $[\mathbf{3 0}]$ (et même dans $[\mathbf{4 7}, \mathbf{4 6}, \mathbf{4 8}]$ ). Cette condition technique demande que les nombres $\left|c_{n, k}\right|^{2}$, d'un même paquet, soient du même ordre de grandeur, c'est-à-dire comparable à leur moyenne $\frac{1}{d_{n}}\left(\left|c_{n, 1}\right|^{2}+\cdots+\left|c_{n, d_{n}}\right|^{2}\right)$. Ainsi, (14) se réduit à

$$
\left\|\sum_{n \in \mathbb{N}} \frac{1}{\operatorname{dim}\left(E_{d, n}\right)}\left(\sum_{k=1}^{d_{n}}\left|c_{n, k}\right|^{2}\right)\left(\sum_{k=1}^{d_{n}}\left|\phi_{n, k}(x)\right|^{2}\right)\right\|_{L_{x}^{p / 2}\left(\mathbb{R}^{d}\right)},
$$

où la fonction $\left|\phi_{n, 1}\right|^{2}+\cdots+\left|\phi_{n, d_{n}}\right|^{2}$ s'avère indépendante de la base hilbertienne choisie. Il est naturel de vouloir s'émanciper de la squeezing condition (cette dernière a été légèrement relaxée dans [30, Part 1.1.2] par comparaison aux travaux $[\mathbf{4 7}, \mathbf{4 6}, \mathbf{4 8}])$. Dans notre article, nous allons employer une autre méthode de randomisation qui ne dépend que de la suite $\left(E_{d, n}\right)_{n>0}$ et qui ne nécessite aucune condition de contrôle des coefficients. De façon précise, on va randomiser une fonction propre appartenant à $E_{d, n}$ en la faisant tourner uniformément et aléatoirement autour de l'origine. Nous noterons désormais $\left(W_{n}\right)_{n \geq 0}$ une suite de matrices aléatoires indépendantes et supposons que chaque matrice aléatoire $W_{n}$ suit une loi uniforme dans le groupe unitaire $U_{d_{n}}(\mathbb{C})$. Notre résultat s'énonce comme suit :

Théorème 1.4. - Supposons $d \geq 2$ et considérons une suite $\left(u_{n}\right)_{\in \mathbb{N}}$ d'éléments de $L^{2}\left(\mathbb{R}^{d}\right)$ vérifiant $u_{n} \in$ $E_{d, n}$ pour tout $n \in \mathbb{N}$. Pour tout réel $p \in[1,+\infty[$, les conditions suivantes sont équivalentes :

i) la série aléatoire $\sum_{n}\left(\sum_{i, j=1}^{d_{n}} W_{n, i, j}\left\langle u_{n}, \phi_{n, j}\right\rangle \phi_{n, i}\right)$ converge presque sûrement dans $L^{p}\left(\mathbb{R}^{d}\right)$,

ii) la série numérique $\sum_{n \geq 1} n^{\frac{d}{2}-1}\left(\sum_{k \geq n} \frac{\left\|u_{k}\right\|_{L^{2}\left(\mathbb{R}^{d}\right)}^{2}}{k^{\frac{d}{2}}}\right)^{\frac{p}{2}}$ est convergente. 
Pour tout $p \in[2,+\infty[$ on a l'injection de Sobolev probabiliste

(16) $\sum_{n \geq 1} n^{-d\left(\frac{1}{2}-\frac{1}{p}\right)}\left\|u_{n}\right\|_{L^{2}\left(\mathbb{R}^{d}\right)}^{2}<+\infty \Rightarrow \sum_{n}\left(\sum_{i, j=1}^{d_{n}} W_{n, i, j}\left\langle u_{n}, \phi_{n, j}\right\rangle \phi_{n, i}\right)$ converge p.s. dans $L^{p}\left(\mathbb{R}^{d}\right)$.

Le théorème 1.4 donne donc une caractérisation explicite des séries aléatoires qui convergent presque sûrement dans $L^{p}\left(\mathbb{R}^{d}\right)$. Au passage, on obtient un résultat de type Paley-Zygmund :

$$
\sum_{n \geq 1}\left\|u_{n}\right\|_{L^{2}\left(\mathbb{R}^{d}\right)}^{2}<+\infty \Rightarrow \forall p \in\left[2,+\infty\left[\sum_{n}\left(\sum_{i, j=1}^{d_{n}} W_{n, i, j}\left\langle u_{n}, \phi_{n, j}\right\rangle \phi_{n, i}\right) \text { converge p.s. dans } L^{p}\left(\mathbb{R}^{d}\right)\right. \text {. }\right.
$$

Dans la partie 4, nous verrons que l'implication (16) est optimale par rapport à l'exposant $-d\left(\frac{1}{2}-\frac{1}{p}\right)$ et doit être vue comme une amélioration probabiliste de l'injection de Sobolev déterministe :

$$
\sum_{n \geq 1} n^{d\left(\frac{1}{2}-\frac{1}{p}\right)}\left\|u_{n}\right\|_{L^{2}\left(\mathbb{R}^{d}\right)}^{2}<+\infty \Rightarrow \sum_{n \geq 0} u_{n} \in L^{p}\left(\mathbb{R}^{d}\right)
$$

Dans [30, Theorem 2.2], un cas particulier de (16) a été obtenu avec une squeezing condition. Nous écrirons aussi une implication duale à celle de (16), avec $p \in] 1,2]$, grâce à un nouveau théorème de dualité.

$\mathrm{Au}$ niveau probabiliste, la preuve du théorème 1.4 reposera sur une extension multidimensionnelle du critère de Maurey (1) qui fera apparaître l'expression (15). An niveau de l'analyse, nous utiliserons une nouvelle estimée de la fonction spectrale de l'oscillateur harmonique tronquée au $n$-ième niveau d'énergie (voir la proposition 4.1) :

$$
\sum_{\substack{\left(i_{1}, \ldots, i_{d}\right) \in \mathbb{N}^{d} \\ i_{1}+\cdots+i_{d}=n}} h_{i_{1}}\left(x_{1}\right)^{2} \ldots h_{i_{d}}\left(x_{d}\right)^{2} .
$$

Didier Robert nous a signalé après la rédaction de notre article que des informations complémentaires sur la concentration de (18) ont été précédemment obtenues dans [28].

1.4. Version multidimensionnelle du théorème de Maurey-Pisier. - Le théorème de MaureyPisier permet de remplacer les variables gaussiennes par des variables de Bernoulli dans les théorèmes 1.1 et 1.2. Il y a en réalité un phénomène d'universalité plus important : on peut remplacer les variables gaussiennes par toute une famille de variables aléatoires ayant des moments finis de tout ordre. Pour énoncer précisément ce résultat, rappelons la définition du cotype.

Définition 1.5. - Considérons un réel $q \in[2,+\infty[$, un espace de Banach complexe $B$ est de cotype $q$ s'il existe $c>0$ tel que pour tout $N \in \mathbb{N}^{\star}$ et tout $\left(u_{n}\right)_{1 \leq n \leq N} \in B^{N}$ on a

$$
\left(\sum_{n=1}^{N}\left\|u_{n}\right\|_{B}^{q}\right)^{1 / q} \leq c \mathbf{E}\left[\left\|\sum_{n=1}^{N} \varepsilon_{n} u_{n}\right\|_{B}\right] .
$$

S'il existe un réel $q \in[2,+\infty[$ tel que l'inégalité précédente se réalise, alors on dit que $B$ est de cotype fini.

Il faut considérer la propriété de cotype fini comme une propriété géométrique d'un espace de Banach. On vérifie que $L^{p}(X)$ est un espace de Banach de cotype $\max (2, p)$. La preuve du théorème de Maurey-Pisier [41, corollaire 1.3] donne en fait le résultat suivant.

Théorème 1.6 (Maurey-Pisier). — Soit B un espace de Banach complexe. Alors les deux propriétés suivantes sont équivalentes :

a) $B$ est de cotype fini,

b) pour toute suite $\left(u_{n}\right)$ de $B$ et toute suite $\left(X_{n}\right)_{n \in \mathbb{N}}$ de variables aléatoires réelles, centrées, indépendantes et vérifiant

les deux propriétés suivantes sont équivalentes

$$
\inf _{n \in \mathbb{N}} \mathbf{E}\left[\left|X_{n}\right|\right]>0 \quad \text { et } \quad \forall q \in\left[2,+\infty\left[\sup _{n \in \mathbb{N}} \mathbf{E}\left[\left|X_{n}\right|^{q}\right]<+\infty,\right.\right.
$$

i) la série aléatoire $\sum X_{n} u_{n}$ converge presque sûrement dans $B$,

ii) la série aléatoire $\sum \varepsilon_{n} u_{n}$ converge presque sûrement dans $B$. 
En d'autres termes, l'universalité de la randomisation unidimensionnelle dans $L^{p}(X)$ se produit précisément parce que $L^{p}(X)$ dispose de la propriété de cotype fini. On veut naturellement généraliser ce phénomène pour les séries aléatoires qui apparaissent dans l'hypothèse i) du théorème 1.4, c'est-à-dire trouver d'autres séries aléatoires multidimensionnelles pour lesquelles la conclusion du théorème 1.4 est encore valide.

Ce problème est lié à un fait remarqué par Burq-Lebeau dans $[\mathbf{9}$, Appendice $\mathrm{C}]$ : si l'on considère $X$ une variété riemannienne compacte sans bord alors on peut construire beaucoup de mesures $\nu$ sur $L^{2}(X)$ (même étrangères deux à deux) telles que $\nu\left(L^{p}(X)\right)=1$ pour tout $p \in[2,+\infty[$. Rappelons simplement comment sont construites les mesures de Burq-Lebeau, disons dans le cas $X=\mathbb{S}^{d}$ (voir [9, Appendice C.1]). Notons $\left(E_{n}\right)_{n \geq 0}$ la suite des sous-espaces propres de l'opérateur de Laplace-Beltrami $\Delta$ sur $L^{2}\left(\mathbb{S}^{d}\right)$ et considérons une suite $\left(V_{n}\right)_{n \geq 1}$ de variables aléatoires indépendantes à valeurs dans ]0, $+\infty$ [ vérifiant les estimations de grandes déviations

$$
\exists \gamma>0 \quad \exists C>0 \quad \exists c>0 \quad \forall \rho>0 \quad \sup _{n \in \mathbb{N}} \mathbf{P}\left(V_{n} \geq \rho\right) \leq C e^{-c \rho^{\gamma}} .
$$

On supposera que les variables aléatoires $V_{n}$ et les matrices unitaires aléatoires $W_{n}$ sont indépendantes dans leur ensemble. Considérons maintenant une suite $\left(u_{n}\right)_{n \geq 1}$ de $L^{2}\left(\mathbb{S}^{d}\right)$ vérifiant $u_{n} \in E_{n}$ pour tout $n \in \mathbb{N}$. Les mesures de Burq-Lebeau sont tout simplement les mesures images (c'est-à-dire les lois) des fonctions aléatoires

$$
\begin{aligned}
\Omega & \rightarrow L^{2}\left(\mathbb{S}^{d}\right) \\
\omega & \mapsto \sum_{n \in \mathbb{N}}\left(\sum_{i, j=1}^{d_{n}} V_{n}(\omega) W_{n, i, j}(\omega)\left\langle u_{n}, \phi_{n, j}\right\rangle \phi_{n, i}\right),
\end{aligned}
$$

où $\left(\phi_{n, 1}, \ldots, \phi_{n, d_{n}}\right)$ est une base orthonormée du sous-espace propre $E_{n}$ et $\Omega$ un univers probabilisé de référence. Par indépendance des variables aléatoires en jeu, cette construction équivaut à définir une mesure de probabilité sur $L^{2}\left(\mathbb{S}^{d}\right)$ comme un produit tensoriel infini de certaines mesures de probabilités respectivement supportées dans les sous-espaces $E_{n}$ (voir $[\mathbf{9}$, pages 955$]$ ). En termes de séries aléatoires, le résultat de Burq-Lebeau se reformule donc ainsi : il existe beaucoup de séries aléatoires de la forme (21) qui convergent presque sûrement dans $L^{p}\left(\mathbb{S}^{d}\right)$. Ce résultat n'est pas purement théorique, il fait partie d'un programme de recherche consistant à étudier le transfert de mesures par le flot d'équations aux dérivées partielles non linéaires (par exemple des mesures de Gibbs).

On va écrire une version multidimensionnelle du théorème 1.6 qui va expliquer pourquoi l'on peut bien construire des mesures de Burq-Lebeau (et au passage en construire des nouvelles). Afin de recouvrir les variables aléatoires $X_{n}$ du théorème 1.6, nous allons considérer des matrices aléatoires plus générales. Introduisons la notion suivante qui généralise la notion de symétrie pour les variables aléatoires scalaires [39, page 82 , line $(2.3)]$.

Définition 1.7. - Une matrice aléatoire $M: \Omega \rightarrow \mathcal{M}_{d}(\mathbb{R})$ est orthogonalement invariante si pour toute matrice orthogonale $P \in O_{d}(\mathbb{R})$ les matrices aléatoires $M$ et $P M$ ont la même loi. On définit de même l'invariance unitaire pour une matrice aléatoire $M: \Omega \rightarrow \mathcal{M}_{d}(\mathbb{C})$ en remplaçant le groupe orthogonal $O_{d}(\mathbb{R})$ par le groupe unitaire $U_{d}(\mathbb{C})$.

Fixons maintenant quelques notations usuelles d'algèbre linéaire :

$$
\begin{aligned}
\forall y \in \mathcal{M}_{d, 1}(\mathbb{C})=\mathbb{C}^{d}|y| & :=\sqrt{\left|y_{1}\right|^{2}+\cdots+\left|y_{d}\right|^{2}}, \\
\forall A \in \mathcal{M}_{d}(\mathbb{C}) \quad\|A\|_{o p} & :=\sup _{y \in \mathbb{C}^{d} \backslash\{0\}} \frac{|A y|}{|y|}, \\
|A| & :=\sqrt{{ }^{t} \bar{A} A} \in \mathcal{M}_{d}(\mathbb{C}), \\
\sigma(A) & :=\inf _{y \neq 0} \frac{|A y|}{|y|} .
\end{aligned}
$$

La matrice $|A|$ est une matrice hermitienne positive issue d'une décomposition polaire de $A=P|A|$ avec $P \in U_{d}(\mathbb{C})$ (une telle décomposition est unique dès lors que $\operatorname{det}(A) \neq 0$ ). Enfin, $\sigma(A)$ est la plus petite valeur singulière de $A$, il s'agit aussi de la plus petite valeur propre de $|A|$. Notre première version multidimensionnelle du théorème de Maurey-Pisier prend la forme suivante : 
Théorème 1.8. - Fixons $X$ un espace mesuré $\sigma$-fini et un réel $p \in[1,+\infty[$. Considérons une suite de sous-espaces non nuls $\left(E_{n}\right)_{n \geq 0}$ de $L^{2}(X) \cap L^{p}(X)$ de dimension finie. On notera $d_{n}=\operatorname{dim}\left(E_{n}\right)$ et $\left(\phi_{n, 1}, \ldots, \phi_{n, d_{n}}\right)$ une base hilbertienne de $E_{n}$. Considérons aussi une suite de matrices aléatoires $M_{n}: \Omega \rightarrow$ $\mathcal{M}_{d_{n}}(\mathbb{R})$ indépendantes et orthogonalement invariantes telles que

$$
\inf _{n \in \mathbb{N}} \sigma\left(\mathbf{E}\left[\left|M_{n}\right|\right]\right)>0 \quad \text { et } \sup _{n \in \mathbb{N}} \mathbf{E}\left[\left\|M_{n}\right\|_{o p}^{\max (2, p)}\right]<+\infty .
$$

Alors, pour toute suite $\left(u_{n}\right)_{\in \mathbb{N}}$, avec $u_{n} \in E_{n}$ pour tout $n \in \mathbb{N}$, les assertions suivantes sont équivalentes :

i) la série aléatoire $\sum_{n}\left(\sum_{i, j=1}^{d_{n}} M_{n, i, j}\left\langle u_{n}, \phi_{n, j}\right\rangle \phi_{n, i}\right)$ converge presque sûrement dans $L^{p}\left(\mathbb{R}^{d}\right)$,

ii) la série aléatoire $\sum_{n}\left(\sum_{i, j=1}^{d_{n}} W_{n, i, j}\left\langle u_{n}, \phi_{n, j}\right\rangle \phi_{n, i}\right)$ converge presque sûrement dans $L^{p}\left(\mathbb{R}^{d}\right)$.

Dans ce qui précède, on convient que $W_{n}: \Omega \rightarrow U_{d_{n}}(\mathbb{C})$ suit une loi uniforme dans le groupe unitaire et que les matrices aléatoires $\left(W_{n}\right)_{n \in \mathbb{N}}$ sont indépendantes.

La même conclusion est valide en remplaçant "orthogonalement invariante" par "unitairement invariante" au sens de la définition 1.7 .

Faisons quelques remarques :

a) L'assertion (20) est beaucoup plus restrictive que la condition $\sup \mathbf{E}\left[\left|V_{n}\right|^{\max (2, p)}\right]<+\infty$. Le théorème 1.8 permet donc de construire des mesures de Burq-Lebeau sur $L^{2}\left(\mathbb{S}^{d}\right)$ qui ne sont pas accessibles par les arguments de grandes déviations utilisés dans $[\mathbf{9}$, appendice $\mathrm{C}]$.

b) Le théorème 1.8 est indépendant d'une quelconque géométrie riemannienne sur $X$. En un certain sens, on peut dire que la philosophie globale est que c'est la géométrie de l'espace de Banach $L^{p}(X)$ qui est déterminante et non la géométrie de l'espace $X$. Cela explique au passage pourquoi les mesures de Burq-Lebeau [9] peuvent être construites sur toute variété riemannienne compacte sans bord.

c) La série aléatoire de l'assertion i) du théorème 1.8 traduit l'idée que l'on applique un endomorphisme aléatoire à l'élément $u_{n} \in E_{n}$. Il s'agit d'une généralisation naturelle des séries aléatoires $\sum X_{n} u_{n}$ où $X_{n}: \Omega \rightarrow \mathbb{R}$ sont des variables aléatoires réelles.

d) Les conditions (23) apparaissent sous une forme analogue dans l'étude des séries de Fourier aléatoires dans l'espace fonctionnel $L^{\infty}(G)$ sur un groupe compact $G$ mais $\left\|M_{n}\right\|_{o p}^{\max (2, p)}$ est remplacé par $\left\|M_{n}\right\|_{o p}^{2}$ (voir [39, page 97, Theorem 3.5]). Dans le cas où le groupe $G$ est abélien et localement compact, Marcus et Pisier expliquent que l'exposant 2 est dû au fait que l'espace des fonctions "presque sûrement continues" sur $G$ est curieusement de cotype 2 (alors que $L^{\infty}(G)$ n'est même pas de cotype fini dès que $G$ est infini).

Notre preuve du théorème 1.8 n'est pas un prolongement de celle du théorème de Maurey-Pisier [41, corollaire 1.3]. La preuve originelle utilise le théorème de factorisation de Pietsch tandis que notre preuve est plus simple et repose essentiellement sur le théorème de Fubini-Tonelli et les inégalités de Kahane-KhintchineMarcus-Pisier. En fait, l'auteur ignore s'il est possible de démontrer le théorème 1.8 avec un théorème de factorisation. L'obstacle est le suivant : on veut démontrer un résultat de nature multidimensionnelle alors que la définition même d'un espace de Banach de cotype fini fait intervenir la randomisation unidimensionnelle (voir le membre droit de (19)). Pour autant, l'exposant $\max (2, p)$, qui apparaît dans (23), est le cotype de l'espace $L^{p}(X)$. Il est donc normal d'interpréter la preuve du théorème 1.8 en termes de cotype. Il se trouve que l'on peut contourner l'obstacle mentionné en considérant la structure de treillis de $L^{p}(X)$ (c'est-à-dire l'existence d'une relation d'ordre compatible avec la structure d'espace de Banach). Dans ce cas, il est connu que la propriété de "cotype fini" (19) peut être définie de manière déterministe sans variables de Bernoulli (qui sont par nature unidimensionnelle). Voici notre version multidimensionnelle du théorème 1.6 qui donne un éclairage sur les raisons géométriques de l'universalité de la randomisation multidimensionnelle dans les espaces de Banach $L^{p}(X)$, avec $p \in[1,+\infty[$.

Théorème 1.9. - Soit B un treillis de Banach complexe, alors se valent

a) l'espace de Banach $B$ est de cotype fini, 
b) pour toute suite d'entiers non nuls $\left(d_{n}\right)_{n \geq 0}$, pour toute suite de matrices aléatoires $M_{n}: \Omega \rightarrow \mathcal{M}_{d_{n}}(\mathbb{R})$ indépendantes, orthogonalement invariantes et vérifiant

$$
\inf _{n \in \mathbb{N}} \sigma\left(\mathbf{E}\left[\left|M_{n}\right|\right]\right)>0 \quad \text { et } \quad \forall q \in\left[2,+\infty\left[\sup _{n \in \mathbb{N}} \mathbf{E}\left[\left\|M_{n}\right\|_{o p}^{q}\right]<+\infty\right.\right.
$$

et pour toute suite de matrices $b_{n} \in \mathcal{M}_{d_{n}}(B)$, les deux propriétés suivantes sont équivalentes

i) la série aléatoire $\sum \operatorname{tr}\left(M_{n} b_{n}\right)$ converge presque sûrement dans $B$,

ii) la série aléatoire $\sum \operatorname{tr}\left(W_{n} b_{n}\right)$ converge presque sûrement dans $B$.

La même conclusion est valide en remplaçant "orthogonalement invariante" par "unitairement invariante" au sens de la définition $1 . \%$.

Le théorème 1.6 fournit évidemment le sens b) $\Rightarrow$ a) en choisissant $d_{n}=1$ pour tout $n \in \mathbb{N}$. C'est donc le sens a $\Rightarrow \mathrm{b}$ ) qui est nouveau (et c'est en fait le sens pratique). On retrouve les séries aléatoires du théorème 1.8 avec $b_{n}=\left[\left\langle u_{n}, \phi_{n, i}\right\rangle \phi_{n, j}\right] \in \mathcal{M}_{d_{n}}\left(L^{p}(X)\right)$. La forme du théorème 1.9 nous a été inspirée par l'étude des séries aléatoires sur un groupe compact dans le livre de Marcus-Pisier [39].

Signalons que certains espaces de Lorentz fournissent des exemples non triviaux de treillis de Banach de cotype fini [16]. À l'instar du théorème 1.8, le théorème 2.21 donnera une version quantitative de l'hypothèse (24) afin de montrer que le bon exposant $q$ à choisir est lié à la $q$-concavité du treillis $B$ et que l'on peut effectivement choisir $q=\max (2, p)$ dans le cas de $B=L^{p}(X)$.

Le résultat suivant, prouvé dans les appendices $\mathrm{B}$ et $\mathrm{C}$, donne des exemples de matrices aléatoires vérifiant les hypothèses (23) et (24).

Proposition 1.10. - Considérons des variables aléatoires $X_{i j}: \Omega \rightarrow \mathbb{R}$ centrées, i.i.d. et où $(i, j)$ parcourt $\mathbb{N}^{\star 2}$. Pour tout $n \in \mathbb{N}^{\star}$, on note la matrice aléatoire $M_{n}=\frac{1}{\sqrt{n}}\left[X_{i j}\right]$. Il existe une constante universelle $C \geq 1$ telle que si l'on a $0<\mathbf{E}\left[\left|X_{1,1}\right|^{4}\right]<+\infty$ alors

$$
\sigma\left(\mathbf{E}\left[\left|M_{n}\right|\right]\right) \geq \frac{\mathbf{E}\left[\left|X_{11}\right|\right]^{2}}{C \mathbf{E}\left[\left|X_{11}\right|^{4}\right]^{\frac{1}{4}}} .
$$

En outre, si $X_{1,1}$ admet un moment d'ordre $p \geq 4$ alors nous avons l'inégalité de Latata précisée

$$
\mathbf{E}\left[\left\|M_{n}\right\|_{o p}^{p}\right] \leq C(p) \mathbf{E}\left[\left|X_{1,1}\right|^{p}\right] .
$$

Par conséquent, on a

$$
\inf _{n \geq 1} \sigma\left(\mathbf{E}\left[\left|M_{n}\right|\right]\right)>0 \quad \text { et } \sup _{n \geq 1} \mathbf{E}\left[\left\|M_{n}\right\|_{o p}^{p}\right]<+\infty
$$

Remarque 1.11. - Si les variables aléatoires $X_{i j}$ sont à valeurs complexes et d'espérance nulle, alors on peut aussi montrer (26) en considérant partie réelle et partie imaginaire des variables aléatoires $X_{i j}$. Quant à (25), une preuve similaire est valide mais l'on doit remplacer l'hypothèse "centrée" par "symétrique au sens complexe" : pour tout réel $\alpha \in \mathbb{R}$ les variables $e^{i \alpha} X_{i j}$ et $X_{i j}$ ont la même loi.

Remarque 1.12. - Dans le cadre des théorèmes 1.8 et 1.9, nous avons besoin de matrices aléatoires orthogonalement invariantes. Or les matrices $\frac{1}{\sqrt{n}}\left[X_{i j}\right]$ de la proposition 1.10 ne sont pas orthogonalement invariantes en général (le cas gaussien est une exception notable). Il suffit alors d'examiner des matrices aléatoires de la forme $\frac{1}{\sqrt{n}} \mathcal{E}_{n}\left[X_{i j}\right]$ où les matrices aléatoires $\mathcal{E}_{n}: \Omega \rightarrow O_{d_{n}}(\mathbb{R})$ et $\left[X_{i j}\right]$ sont indépendantes et où $\mathcal{E}_{n}$ suit une loi uniforme pour chaque $n \in \mathbb{N}^{\star}$. On remarque évidemment que $\frac{1}{\sqrt{n}} \mathcal{E}_{n}\left[X_{i j}\right]$ et $\frac{1}{\sqrt{n}}\left[X_{i j}\right]$ ont la même norme d'opérateur et que l'on a $\left|\mathcal{E}_{n}\left[X_{i j}\right]\right|=\left|\left[X_{i j}\right]\right|$ (au sens de (22)).

Nous n'avons pas trouvé les estimations de la proposition 1.10 dans des références déjà publiées mais elles doivent être connues des spécialistes. Par exemple, nous pensons qu'il doit être possible de prouver (26) avec la méthode des moments (par exemple expliquée dans [55, Part 2.3]). Cependant, cette méthode est assez technique à mettre en place. Un cas particulier très bien compris dans la littérature des matrices aléatoires est le cas sous-gaussien. Parmi plusieurs définitions équivalentes, $X_{1,1}$ est sous-gaussienne si l'on a $\mathbf{E}\left[X_{1,1}\right]=0$ et

$$
\exists K>0 \quad \forall p \in \mathbb{N}^{\star} \quad \mathbf{E}\left[\left|X_{1,1}\right|^{p}\right]^{\frac{1}{p}} \leq K \sqrt{p} .
$$


Cela équivaut par exemple à la condition

$$
\exists K^{\prime}>0 \quad \forall t \in \mathbb{R} \quad \mathbf{E}\left[\exp \left(t X_{11}\right)\right] \leq \exp \left(K^{\prime} t^{2}\right) .
$$

D'après [49, Proposition 2.3] (voir aussi la preuve de [38, Fact 2.4]), on sait que l'on a

$$
\forall p \in \mathbb{N}^{\star} \quad \exists C(p, K)>0 \quad \forall n \in \mathbb{N}^{\star} \quad \mathbf{E}\left[\left\|M_{n}\right\|_{o p}^{p}\right] \leq C(p, K) .
$$

L’inégalité (26) est plus générale. Dans notre article, nous montrons que (26) est en réalité une conséquence de la preuve de l'inégalité de Latała

$$
\mathbf{E}\left[\left\|M_{n}\right\|_{o p}\right] \leq C \mathbf{E}\left[\left|X_{1,1}\right|^{4}\right]^{\frac{1}{4}}
$$

et des inégalités de Kahane-Khintchine dans l'espace de Banach $\left(\mathcal{M}_{n}(\mathbb{R}),\|\cdot\|_{o p}\right)$ (voir (39)). Cela explique pourquoi $(26)$ implique $(27)$ avec $p=4$. La renormalisation de $\left[X_{i j}\right]$ par le facteur $\frac{1}{\sqrt{n}}$ et le fait que $(27)$ nécessite au moins un moment d'ordre 4 peuvent surprendre a priori mais il s'agit de faits bien connus dans la théorie des matrices aléatoires (voir [2] et [62, Theorem 3.1]). Enfin, l'inégalité triviale $\left|X_{1,1}\right| \leq \sqrt{n}\left\|M_{n}\right\|_{o p}$ nous fait comprendre que l'inégalité (26) est optimale par rapport à la condition de moment d'ordre $p$.

Quant à l'inégalité (25), elle découlera d'un argument d'interpolation simplifiant celui du cas gaussien [39, page 78, Proposition 1.5] et de l'inégalité de Latała (27).

1.5. Organisation de l'article. — La partie 2 contient les preuves des théorèmes 1.8 et 1.9. Mais elle est plus généralement dévolue à l'étude abstraite des espaces de Lebesgue probabilistes, notés $\mathbf{P} \mathbf{L}^{p}\left(X, \oplus E_{n}\right)$ où $X$ est un espace mesuré $\sigma$-fini et une suite $\left(E_{n}\right)_{n \in \mathbb{N}}$ de sous-espaces non nuls de dimension finie de $L^{2}(X)$. Expliquons brièvement la définition de ces nouveaux espaces de Banach. Notons $\left(\phi_{n, 1}, \ldots, \phi_{n, d_{n}}\right)$ une base orthonormée de $E_{n}$ pour tout $n \in \mathbb{N}$. Pour tout $p \in\left[1,+\infty\left[\right.\right.$, l'espace $\mathbf{P L}^{p}\left(X, \oplus E_{n}\right)$ sera défini comme l'espace vectoriel des suites $\left(u_{n}\right)_{n \in \mathbb{N}}$, avec $u_{n} \in E_{n}$ pour tout $n \in \mathbb{N}$, telles que la série aléatoire

$$
\sum_{n}\left(\sum_{i, j=1}^{d_{n}} W_{n, i, j}\left\langle u_{n}, \phi_{n, j}\right\rangle \phi_{n, i}\right)
$$

converge presque sûrement dans $L^{p}(X)$. Le cas $\operatorname{dim}\left(E_{n}\right)=1$, pour tout $n \in \mathbb{N}$, est le plus simple à comprendre grâce au théorème de Maurey-Pisier et au critère de Maurey (1). Pour analyser les séries aléatoires $(28)$ dans le cas $\operatorname{dim}\left(E_{n}\right) \neq 1$, nous allons utiliser une version multidimensionnelle des inégalités de Kahane-Khintchine, à savoir les inégalités de Kahane-Khintchine-Marcus-Pisier que nous avons mentionnées plus haut. L'idée maîtresse de notre article est que ces inégalités sont précisément celles qui permettent d'aborder de façon satisfaisante la randomisation multidimensionnelle avec des hypothèses optimales de moments. Le théorème 2.2 nous donnera notamment une extension multidimensionnelle du critère de Maurey avec la caractérisation suivante des espaces de Lebesgue probabilistes :

$$
\left(u_{n}\right)_{n \in \mathbb{N}} \in \mathbf{P L}^{p}\left(X, \oplus E_{n}\right) \Leftrightarrow\left\|\sqrt{\sum_{n \in \mathbb{N}}\left\|u_{n}\right\|_{L^{2}(X)}^{2} \frac{e_{n}(x)}{\operatorname{dim}\left(E_{n}\right)}}\right\|_{L_{x}^{p}(X)}<+\infty
$$

où la fonction $e_{n}:=\left|\phi_{n, 1}\right|^{2}+\cdots+\left|\phi_{n, d_{n}}\right|^{2}$ est indépendante de la base hilbertienne $\left(\phi_{n, 1}, \ldots, \phi_{n, d_{n}}\right)$ (voir $(33))$.

A priori, on s'attend à ce que le dual de $\mathbf{P} \mathbf{L}^{p}\left(X, \oplus E_{n}\right)$ soit $\mathbf{P} \mathbf{L}^{\frac{p}{p-1}}\left(X, \oplus E_{n}\right)$ et l'on espère que les espaces $\mathbf{P L}^{p}\left(X, \oplus E_{n}\right)$ soient stables par interpolation complexe et réelle. En fait, cela s'avérera faux pour la randomisation des fonctions zonales sur la sphère $\mathbb{S}^{d}$ (voir la partie 3) : on n'a pas de dualité et l'interpolation se réalise seulement si $p$ parcourt certains intervalles. Nous avons cependant des résultats positifs : les théorèmes 2.5 et 2.6 donnent des hypothèses pratiques sur les fonctions $e_{n}$ qui assurent les propriétés attendues de dualité et d'interpolation. La stratégie consistera à montrer $\mathbf{P L}^{p}\left(X, \oplus E_{n}\right)$ est un rétracte convenable de l'espace de Bochner-Lebesgue $L^{p}\left(X, \ell^{2}(\mathbb{N})\right)$. Cela nous permettra de faire hériter les propriétés de dualité/interpolation des espaces $L^{p}\left(X, \ell^{2}(\mathbb{N})\right)$ aux espaces $\mathbf{P L}^{p}\left(X, \oplus E_{n}\right)$. Mais pour y arriver, nous serons obligés de justifier la continuité de certains projecteurs de $L^{p}\left(X, \ell^{2}(\mathbb{N})\right)$ à l'aide d'un nouveau critère de $\mathcal{R}$-bornitude sur $L^{p}(X)$. Les notions de rétracte et de $\mathcal{R}$-bornitude, que nous rappellerons plus loin, peuvent 
paraître abstraites mais elles sont incontournables sous une forme ou une autre (cela est formalisé par la proposition 2.31). C'est à ce moment qu'interviendra l'hypothèse abstraite

$$
\sup _{n \in \mathbb{N}} \frac{\sqrt{e_{n}}}{\left\|\sqrt{e_{n}}\right\|_{L^{p}(X)}} \in L^{p, \infty}(X)
$$

où $L_{x}^{p, \infty}(X)$ est l'usuel espace $L^{p}$ faible. Curieusement, il se trouve que la propriété (29) est vérifiée dans des exemples importants issus de la physique mathématique où les fonctions $e_{n}$ se concentrent sur une même région de $X$.

La partie 3 contient les preuves des théorèmes 1.1 et 1.2 grâce aux nouveaux arguments d'interpolation. Expliquons la principale idée dans le cas particulier $\operatorname{dim}\left(E_{n}\right)=1$, c'est-à-dire si $E_{n}=\mathbb{C} \phi_{n}$. Supposons en outre que $\left|\phi_{n}\right|$ a tendance à se concentrer, en un sens à préciser, sur une partie $A_{n} \subset X$ avec une amplitude $c_{n}>0$ quand $n$ tend vers $+\infty$. Si l'on fixe une suite de coefficients $\left(a_{n}\right) \in \ell^{2}(\mathbb{N})$, alors il est légitime d'espérer l'équivalence suivante pour toute suite complexe $\left(a_{n}\right)_{n \in \mathbb{N}}$

$$
\sqrt{\sum_{n \in \mathbb{N}}\left|a_{n} \phi_{n}\right|^{2}} \in L^{p}(X) \Leftrightarrow \sqrt{\sum_{n \in \mathbb{N}}\left|a_{n} c_{n} \mathbf{1}_{A_{n}}\right|^{2}} \in L^{p}(X) .
$$

En partitionnant les parties $A_{n}$ en sous-parties disjointes, la condition du membre droit est explicite et résoudrait le problème de l'étude de la série aléatoire $\sum \varepsilon_{n} a_{n} \phi_{n}$. Si l'approximation de $\left|\phi_{n}\right| \operatorname{par} c_{n} \mathbf{1}_{A_{n}}$ n'est pas bonne, alors l'équivalence précédente n'est pas facile à prouver. L'hypothèse (29), avec $e_{n}=\left|\phi_{n}\right|^{2}$, a vocation à mesurer le reste de cette approximation et les arguments de dualité-interpolation permettent de simplifier les calculs.

La partie 4 est consacrée à la preuve du théorème 1.4 concernant l'oscillateur harmonique multidimensionnel.

Nous ajoutons trois appendices. Le premier est consacré à l'optimalité de l'exposant max $(2, p)$ dans les différents théorèmes concernant $L^{p}(X)$ (ce fait est sans doute bien connu). Les deux derniers appendices contiennent la preuve des estimations matricielles de la proposition 1.10 .

Conventions. Dans cet article, on fera les conventions suivantes

- sauf exception, la lettre $d$ sera globalement réservée pour désigner la dimension des espaces en jeu $\left(\mathbb{R}^{d}\right.$ ou $\left.\mathbb{S}^{d}\right)$ et sera supérieure ou égale à 2 .

- le triplet $(\Omega, \mathcal{F}, \mathbf{P})$ désignera un espace probabilisé de référence (par exemple $\Omega=[0,1]$ muni de la mesure de Lebesgue sur la tribu borélienne), on notera $\omega$ les éléments de $\Omega$ et $\mathbf{E}$ l'opérateur d'espérance.

- on notera $C$ une constante universelle supérieure ou égale à 1 qui peut changer d'une ligne à l'autre.

- on notera $C(d, p)$ une constante supérieure ou égale à 1 qui ne dépend que des paramètres $d$ et $p$ et qui peut changer d'une ligne à l'autre.

- si $A$ et $B$ sont deux nombres réels, la formule $A \lesssim B$ signifiera qu'il existe une constante universelle $C \geq 1$ telle que $A \leq C B$. De même, l'assertion $A \gtrsim B$ signifie $B \lesssim A$.

- on écrira $A \simeq B$ si l'on a $A \lesssim B$ et $B \lesssim A$.

- si l'on fait intervenir des paramètres $d$ et $p$, alors on notera $A \lesssim d, p \quad B$ pour signifier qu'il existe une constante $C(d, p) \geq 1$ qui ne dépend que de $d$ et $p$ telle que $A \leq C(d, p) B$. On définit de même les symboles $\gtrsim_{d, p}$ et $A \simeq_{d, p}$.

Par exemple, nous avons l'équivalence

$$
\forall n \in \mathbb{N} \quad A_{n} \simeq_{d, p} B_{n} \quad \Leftrightarrow \quad \exists C(d, p) \geq 1 \quad \forall n \in \mathbb{N} \quad \frac{A_{n}}{C(d, p)} \leq B_{n} \leq C(d, p) A_{n} .
$$

\section{Espaces de Lebesgue probabilistes}

2.1. Universalité de la randomisation multidimensionnelle dans $L^{p}$ - — On énonce les résultats qui vont impliquer le théorème 1.8 et nous seront utiles pour la suite. On utilisera la notation suivante pour la norme matricielle de Hilbert-Schmidt :

$$
\forall A \in \mathcal{M}_{N}(\mathbb{C}) \quad\|A\|_{H S}:=\sqrt{\sum_{i=1}^{N} \sum_{j=1}^{N}\left|a_{i j}\right|^{2}}
$$


Désormais $X$ désignera un espace mesuré $\sigma$-fini. Nous allons démontrer le théorème suivant.

Théorème 2.1. - Fixons un réel $p \in\left[1,+\infty\left[\right.\right.$, une suite d'entiers non nuls $\left(d_{n}\right)_{n \in \mathbb{N}}$, une suite de de matrices $b_{n} \in \mathcal{M}_{d_{n}}\left(L^{p}(X)\right)$ et une suite de matrices aléatoires $M_{n}: \Omega \rightarrow \mathcal{M}_{d_{n}}(\mathbb{R})$ indépendantes, orthogonalement invariantes et vérifiant

$$
\inf _{n \in \mathbb{N}} \sigma\left(\mathbf{E}\left[\left|M_{n}\right|\right]\right)>0 \quad \text { et } \quad \sup _{n \in \mathbb{N}} \mathbf{E}\left[\left\|M_{n}\right\|_{o p}^{\max (2, p)}\right]<+\infty .
$$

Alors les propriétés suivantes sont équivalentes :

i) la fonction $x \mapsto \sqrt{\sum_{n \geq 0}\left\|b_{n}(x)\right\|_{H S}^{2}}$ appartient à $L^{p}(X)$,

ii) la série aléatoire $\sum \sqrt{d_{n}} \operatorname{tr}\left(M_{n} b_{n}\right)$ converge presque sûrement dans $L^{p}(X)$,

iii) la série aléatoire $\sum \sqrt{d_{n}} \operatorname{tr}\left(M_{n} b_{n}\right)$ converge dans $L^{\max (2, p)}\left(\Omega, L^{p}(X)\right)$,

iv) la série aléatoire $\sum \sqrt{d_{n}} \operatorname{tr}\left(M_{n} b_{n}\right)$ est bornée en probabilité dans $L^{p}(X)$ (voir définition (46) plus loin). La même conclusion est valide en supposant que chaque matrice aléatoire $M_{n}: \Omega \rightarrow \mathcal{M}_{d_{n}}(\mathbb{C})$ est unitairement invariante.

Le théorème 2.1 implique le suivant.

Théorème 2.2. - Fixons un réel $p \in\left[1,+\infty\left[\right.\right.$ et une suite de sous-espaces non nuls $\left(E_{n}\right)_{n \geq 0}$ de $L^{2}(X) \cap$ $L^{p}(X)$ de dimension finie. On notera $d_{n}=\operatorname{dim}\left(E_{n}\right)$ et $\left(\phi_{n, 1}, \ldots, \phi_{n, d_{n}}\right)$ une base hilbertienne de $E_{n}$ pour tout $n \in \mathbb{N}$. On définit de plus

$$
\forall x \in X \quad e_{n}(x)=\left|\phi_{n, 1}(x)\right|^{2}+\cdots+\left|\phi_{n, d_{n}}(x)\right|^{2} .
$$

Fixons de plus une suite de matrices aléatoires $M_{n}: \Omega \rightarrow \mathcal{M}_{d_{n}}(\mathbb{R})$ indépendantes, orthogonalement invariantes et vérifiant

$$
\inf _{n \in \mathbb{N}} \sigma\left(\mathbf{E}\left[\left|M_{n}\right|\right]\right)>0 \quad \text { et } \quad \sup _{n \in \mathbb{N}} \mathbf{E}\left[\left\|M_{n}\right\|_{o p}^{\max (2, p)}\right]<+\infty .
$$

Pour toute suite $\left(u_{n}\right)_{n \geq 0}$, avec $u_{n} \in E_{n}$, on définit la série aléatoire

$$
\sum_{n}\left(\sum_{i, j=1}^{d_{n}} M_{n, i, j}\left\langle u_{n}, \phi_{n, j}\right\rangle \phi_{n, i}\right) \text {. }
$$

Les propriétés suivantes sont alors équivalentes :

i) la fonction $x \mapsto \sqrt{\sum_{n \geq 0}\left\|u_{n}\right\|_{L^{2}(X)}^{2} \frac{e_{n}(x)}{d_{n}}}$ appartient à $L^{p}(X)$,

ii) la série aléatoire (32) converge presque sûrement dans $L^{p}(X)$,

iii) la série aléatoire (32) converge dans l'espace de Bochner-Lebesgue $L^{\max (2, p)}\left(\Omega, L^{p}(X)\right)$,

iv) la série aléatoire (32) est bornée en probabilité dans $L^{p}(X)$.

Le même énoncé est valide si les matrices aléatoires $M_{n}: \Omega \rightarrow \mathcal{M}_{d_{n}}(\mathbb{C})$ sont supposées unitairement invariantes.

Preuve. Il s'agit d'appliquer convenablement le théorème 2.1. Pour tout entier $n \in \mathbb{N}$, on écrit

$$
\sum_{i=1}^{d_{n}} \sum_{j=1}^{d_{n}} M_{n, i, j}\left\langle u_{n}, \phi_{n, j}\right\rangle \phi_{n, i}=\sqrt{d_{n}} \operatorname{tr}\left(M_{n} b_{n}\right),
$$

avec

Cela nous donne

$$
\forall x \in X \quad b_{n}(x):=\frac{1}{\sqrt{d_{n}}}\left[\left\langle u_{n}, \phi_{n, i}\right\rangle \phi_{n, j}(x)\right]_{i, j} \in \mathcal{M}_{d_{n}}\left(L_{x}^{p}(X)\right) .
$$

$$
\left\|b_{n}(x)\right\|_{H S}^{2}=\sum_{i, j=1}^{d_{n}} \frac{1}{d_{n}}\left|\left\langle u_{n}, \phi_{n, i}\right\rangle \phi_{n, j}(x)\right|^{2}=\frac{1}{d_{n}}\left\|u_{n}\right\|_{L^{2}(X)}^{2} \sum_{j=1}^{d_{n}}\left|\phi_{n, j}(x)\right|^{2}=\left\|u_{n}\right\|_{L^{2}(X)}^{2} \frac{e_{n}(x)}{d_{n}} .
$$


La condition i) du théorème 2.2 doit être vue comme une extension multidimensionnelle du critère de Maurey (1) et éclaire la discussion sur la squeezing condition (voir (14) et (15)). Remarquons aussi que la fonction (31) ne dépend que du sous-espace $E_{n}$ en vertu des formules

$$
\begin{aligned}
e_{n}(x) & =\sup _{\left|a_{1}\right|^{2}+\cdots+\left|a_{d_{n}}\right|^{2}=1}\left|a_{1} \phi_{n, 1}(x)+\cdots+a_{d_{n}} \phi_{n, d_{n}}(x)\right|^{2} \\
& =\sup \left\{\left|u_{n}(x)\right|^{2}, \quad u_{n} \in E_{n} \quad\left\|u_{n}\right\|_{L^{2}(X)}=1\right\} .
\end{aligned}
$$

Par conséquent la condition i) est indépendante de la suite des lois des matrices aléatoires $M_{n}$ et des bases hilbertiennes $\phi_{n, 1}, \ldots, \phi_{n, d_{n}}$ de $E_{n}$. Cela traduit précisément un phénomène d'universalité pour la randomisation dans $L^{p}(X)$ et implique le théorème 1.8. L'interprétation avec la notion de cotype sera faite dans la partie 2.4 .

2.2. Interpolation et dualité des espaces de Lebesgue probabilistes $\mathbf{P L}^{p}$ - — Le théorème 2.2 amène à poser la définition suivante.

Définition 2.3. - Fixons un réel $p \in\left[1,+\infty\left[\right.\right.$ et une suite de sous-espaces non nuls $\left(E_{n}\right)_{n \geq 0}$ de $L^{2}(X) \cap$ $L^{p}(X)$ de dimension finie. On notera $d_{n}=\operatorname{dim}\left(E_{n}\right)$ et $\left(\phi_{n, 1}, \ldots, \phi_{n, d_{n}}\right)$ une base hilbertienne de $E_{n}$ pour tout $n \in \mathbb{N}$. On notera aussi

$$
\forall x \in X \quad e_{n}(x)=\left|\phi_{n, 1}(x)\right|^{2}+\cdots+\left|\phi_{n, d_{n}}(x)\right|^{2} .
$$

On note $\mathbf{P L}^{p}\left(X, \oplus E_{n}\right)$ l'espace vectoriel des suites $\left(u_{n}\right)_{n \geq 0}$, avec $u_{n} \in E_{n}$ pour tout $n \geq 0$, qui satisfont les assertions équivalentes $i)$, ii), iii), iv) du théorème 2.2. On appelle $\mathbf{P L}^{p}\left(X, \oplus E_{n}\right)$ l'espace de Lebesgue probabiliste associé à la suite de sous-espaces $\left(E_{n}\right)_{n \geq 0}$ et on le munit de la norme

$$
\left\|\left(u_{n}\right)\right\|_{\mathbf{P L}^{p}\left(X, \oplus E_{n}\right)}:=\left\|\sqrt{\sum_{n \geq 0}\left\|u_{n}\right\|_{L^{2}(X)}^{2} \frac{e_{n}(x)}{\operatorname{dim}\left(E_{n}\right)}}\right\|_{L_{x}^{p}(X)} .
$$

Faisons quelques remarques sur cette définition :

- répétons que la fonction $e_{n}$ est indépendante de la base $\left(\phi_{n, 1}, \ldots, \phi_{n, d_{n}}\right)$.

- on vérifie facilement que $\mathbf{P L}^{p}\left(X, \oplus E_{n}\right)$ est complet (voir (57)).

- bien que défini abstraitement, nous verrons que dans les cas qui nous intéressent, on pourra considérer $\mathbf{P L}^{p}\left(X, \oplus E_{n}\right)$ comme un espace de distributions (par exemple si $X=\mathbb{S}^{d}$ ou $X=\mathbb{R}^{d}$ ).

- si une suite $\left(u_{n}\right)$, vérifiant $u_{n} \in E_{n}$ pour tout $n \in \mathbb{N}$, n'appartient pas à $\mathbf{P} \mathbf{L}^{p}\left(X, \oplus E_{n}\right)$ alors les séries aléatoires $(32)$ du théorème 2.2 divergent dans $L^{p}(X)$ avec une probabilité strictement positive et donc divergent presque sûrement d'après la loi du tout ou rien.

- puisque les fonctions $\frac{1}{d_{n}} e_{n}$ sont des densités de probabilité sur $X$, on a l'égalité

$$
\mathbf{P L}^{2}\left(X, \oplus E_{n}\right)=\left\{\left(u_{n}\right)_{n \in \mathbb{N}}, \quad u_{n} \in E_{n}, \quad \sum_{n \in \mathbb{N}}\left\|u_{n}\right\|_{L^{2}(X)}^{2}<+\infty\right\} .
$$

Si $p$ est différent de 2, alors il semble nécessaire d'exploiter des propriétés des fonctions $e_{n}$ afin de mieux comprendre l'espace $\mathbf{P} \mathbf{L}^{p}\left(X, \oplus E_{n}\right)$. Nous proposons une approche par dualité et interpolation. Le prochain résultat montre que l'interpolation des espaces $\mathbf{P} \mathbf{L}^{p}\left(X, \oplus E_{n}\right)$ n'est pas gratuite et implique des propriétés sur les fonctions $e_{n}$. Rappelons maintenant que les espaces interpolés des espaces $\mathbf{P} \mathbf{L}^{p}\left(X, \oplus E_{n}\right)$ sont définis de façon abstraite mais peuvent être vus comme des sous-espaces vectoriels du même espace ambiant, à savoir $\prod_{n \in \mathbb{N}} E_{n}$. Dans la suite, on notera $[\cdot, \cdot]_{\theta}$ et $[\cdot, \cdot]_{\theta, p}$ les méthodes d'interpolation complexe et réelle.

Proposition 2.4. - Fixons des réels $p_{1}<p<p_{2}$ appartenant à $[1,+\infty[$ et soit $\theta \in] 0,1[$ le nombre défini par la relation $\frac{1}{p}=\frac{1-\theta}{p_{1}}+\frac{\theta}{p_{2}}$. Considérons une suite de sous-espaces non nuls $\left(E_{n}\right)_{n \geq 0}$ de $L^{2}(X) \cap L^{p_{1}}(X) \cap$ $L^{p_{2}}(X)$ de dimension finie et supposons que l'on a l'égalité d'espaces vectoriels

$$
\mathbf{P L}^{p}\left(X, \oplus E_{n}\right)=\left[\mathbf{P L}^{p_{1}}\left(X, \oplus E_{n}\right), \mathbf{P L}^{p_{2}}\left(X, \oplus E_{n}\right)\right]_{\theta}
$$


et que les normes des deux précédents espaces sont équivalentes. Alors

$$
\sup _{n \in \mathbb{N}} \frac{\left\|\sqrt{e_{n}}\right\|_{L^{p_{1}(X)}}^{1-\theta}\left\|\sqrt{e_{n}}\right\|_{L^{p_{2}(X)}}^{\theta}}{\left\|\sqrt{e_{n}}\right\|_{L^{p}(X)}}<+\infty .
$$

La même conclusion est valide en remplaçant la méthode d'interpolation complexe $[\cdot, \cdot]_{\theta}$ par la méthode d'interpolation réelle $[\cdot, \cdot]_{\theta, p}$.

Preuve. On considère pour tout entier $k \in \mathbb{N}$ le "projecteur sur $E_{k}$ " défini par

$$
\begin{aligned}
\Lambda_{k}: \prod_{n \in \mathbb{N}} E_{n} & \rightarrow L^{2}(X) \\
\left(u_{n}\right)_{n \in \mathbb{N}} & \mapsto u_{k} .
\end{aligned}
$$

Le calcul de la norme d'opérateur de $\Lambda_{k}: \mathbf{P L}^{p}\left(X, \oplus E_{n}\right) \rightarrow L^{2}(X)$ est immédiat

$$
\left\|\Lambda_{k}\right\|_{\mathbf{P L}^{p}\left(X, \oplus E_{n}\right) \rightarrow L^{2}(X)}=\sup _{\left(u_{n}\right) \neq 0} \frac{\left\|u_{k}\right\|_{L^{2}(X)}}{\left\|\sqrt{\sum_{n \in \mathbb{N}}\left\|u_{n}\right\|_{L^{2}(X)}^{2} \frac{e_{n}}{d_{n}}}\right\|_{L^{p}(X)}}=\frac{\sqrt{d_{k}}}{\|\sqrt{e(k, \cdot)}\|_{L^{p}(X)}} .
$$

Par interpolation, il existe une constante $K>0$ telle que pour tout $k \in \mathbb{N}$ on a

$$
\left\|\Lambda_{k}\right\|_{\mathbf{P L}^{p}\left(X, \oplus E_{n}\right) \rightarrow L^{2}(X)} \leq K\left\|\Lambda_{k}\right\|_{\mathbf{P L}^{p_{1}}\left(X, \oplus E_{n}\right) \rightarrow L^{2}(X)}^{1-\theta}\left\|\Lambda_{k}\right\|_{\mathbf{P L}^{p_{2}}\left(X, \oplus E_{n}\right) \rightarrow L^{2}(X)}^{\theta} .
$$

L'inégalité (34) renverse l'inégalité usuelle $\left\|\sqrt{e_{n}}\right\|_{L^{p}(X)} \leq\left\|\sqrt{e_{n}}\right\|_{L^{p_{1}(X)}}^{1-\theta}\left\|\sqrt{e_{n}}\right\|_{L^{p_{2}(X)}}^{\theta}$. L'auteur ignore si (34) suffit pour interpoler les espaces $\mathbf{P L}^{p}\left(X, \oplus E_{n}\right)$. Afin d'obtenir des résultats positifs d'interpolation (et même de dualité), nous allons ajouter une hypothèse supplémentaire qui utilise l'espace de Lorentz $L^{1, \infty}(X)$. Il s'agit de l'espace vectoriel des fonctions mesurables $f: X \rightarrow \mathbb{C} \cup\{\infty\}$ telles que

$$
\forall t>0 \quad \mu\{x \in X, \quad|f(x)|>t\}=\mathcal{O}\left(t^{-1}\right),
$$

où $\mu$ est la mesure de l'espace mesuré $X$. L'inclusion $L^{1}(X) \subset L^{1, \infty}(X)$ est toujours vraie et est généralement stricte. Le contre-exemple typique est $|x|^{-d} \in L_{x}^{1, \infty}\left(\mathbb{R}^{d}\right) \backslash L_{x}^{1}\left(\mathbb{R}^{d}\right)$.

Théorème 2.5. - Considérons $p_{1}<p_{2}$ deux nombres appartenant à $] 1,+\infty[$ et vérifiant la condition $\frac{1}{p_{1}}+\frac{1}{p_{2}} \leq 1$. Considérons une suite de sous-espaces non nuls $\left(E_{n}\right)_{n \geq 0}$ de $L^{2}(X) \cap L^{p_{1}}(X) \cap L^{p_{2}}(X)$ de dimension finie. On suppose que les fonctions $e_{n}$ satisfont les propriétés suivantes

$$
\begin{gathered}
\sup _{n \in \mathbb{N}}\left(\frac{\sqrt{e_{n}}}{\left\|\sqrt{e_{n}}\right\|_{L^{p_{1}(X)}}}\right)^{p_{1}}+\sup _{n \in \mathbb{N}}\left(\frac{\sqrt{e_{n}}}{\left\|\sqrt{e_{n}}\right\|_{L^{p_{2}(X)}}}\right)^{p_{2}} \in L^{1, \infty}(X) \\
\left.\exists p_{0} \in\right] p_{1}, p_{2}\left[\sup _{n \in \mathbb{N}} \frac{\left\|\sqrt{e_{n}}\right\|_{L^{p_{1}(X)}}^{1-\theta_{0}}\left\|\sqrt{e_{n}}\right\|_{L^{p_{2}(X)}}^{\theta_{0}}}{\left\|\sqrt{e_{n}}\right\|_{L^{p_{0}(X)}}}<+\infty,\right.
\end{gathered}
$$

où $\left.\theta_{0} \in\right] 0,1\left[\right.$ est déterminé par la relation $\frac{1}{p_{0}}=\frac{1-\theta_{0}}{p_{1}}+\frac{\theta_{0}}{p_{2}}$.

Alors les espaces $\mathbf{P L}^{p}\left(X, \oplus E_{n}\right)$, pour p parcourant $] p_{1}, p_{2}[$, sont stables par interpolation complexe : en d'autres termes, si l'on a

$$
\left.p_{1}<p_{1}^{\prime}<p<p_{2}^{\prime}<p_{2}, \quad \theta \in\right] 0,1\left[, \quad \frac{1}{p}=\frac{1-\theta}{p_{1}^{\prime}}+\frac{\theta}{p_{2}^{\prime}},\right.
$$

alors on a l'égalité $\mathbf{P L}^{p}\left(X, \oplus E_{n}\right)=\left[\mathbf{P L}^{p_{1}^{\prime}}\left(X, \oplus E_{n}\right), \mathbf{P L}^{p_{2}^{\prime}}\left(X, \oplus E_{n}\right)\right]_{\theta}$ avec équivalence des normes.

De même, les espaces $\mathbf{P L}^{p}\left(X, \oplus E_{n}\right)$, pour p parcourant $] p_{1}, p_{2}[$, sont stables par d'interpolation réelle : on a l'égalité $\mathbf{P} \mathbf{L}^{p}\left(X, \oplus E_{n}\right)=\left[\mathbf{P L}^{p_{1}^{\prime}}\left(X, \oplus E_{n}\right), \mathbf{P L}^{p_{2}^{\prime}}\left(X, \oplus E_{n}\right)\right]_{\theta, p}$ avec équivalences des normes. 
La condition $\frac{1}{p_{1}}+\frac{1}{p_{2}} \leq 1$ est de nature technique et est peut-être inutile en toute généralité. Elle est toujours vérifiée si l'on a $2 \leq p_{1}<p_{2}$. Une autre situation intéressante se produit si $p_{1}$ et $p_{2}$ sont deux exposants conjugués, i.e. vérifient $\frac{1}{p_{1}}+\frac{1}{p_{2}}=1$. Dans ce cas, il sera commode de considérer $(36)$ avec $p_{0}=2$ et $\theta=\frac{1}{2}$ pour avoir l'assertion suivante qui est en apparence plus faible (mais équivalente comme le lemme 2.36 le montrera plus loin) :

$$
\sup _{n \in \mathbb{N}} \frac{\left\|\sqrt{e_{n}}\right\|_{L^{p_{1}(X)}}\left\|\sqrt{e_{n}}\right\|_{L^{p_{2}(X)}}}{\operatorname{dim}\left(E_{n}\right)}<+\infty .
$$

En prime de l'interpolation, le théorème suivant donne une propriété de dualité.

Théorème 2.6. - Fixons deux réels $p_{1}<p_{2}$ appartenant à $] 1,+\infty\left[\right.$ et vérifiant l'égalité $\frac{1}{p_{1}}+\frac{1}{p_{2}}=1$. Considérons une suite de sous-espaces non nuls $\left(E_{n}\right)_{n \geq 0}$ de $L^{p_{1}}(X) \cap L^{p_{2}}(X)$ de dimension finie. Pour tous $p \in] p_{1}, p_{2}\left[\right.$ et $(u, w) \in \mathbf{P L}^{p}\left(X, \oplus E_{n}\right) \times \mathbf{P L}^{q}\left(X, \oplus E_{n}\right)$, avec $q:=\frac{p}{p-1}$, on a

$$
\sum_{n \geq 0}\left|\left\langle u_{n}, w_{n}\right\rangle_{L^{2}(X)}\right| \leq\|u\|_{\mathbf{P L}^{p}\left(X, \oplus E_{n}\right)}\|w\|_{\mathbf{P L}^{q}\left(X, \oplus E_{n}\right)}
$$

En d'autres termes, tout élément de $\mathbf{P L}^{q}\left(X, \oplus E_{n}\right)$ induit canoniquement une forme linéaire bornée sur $\mathbf{P L}^{p}\left(X, \oplus E_{n}\right)$.

En outre, si l'on suppose (35) et (37) alors les espaces $\mathbf{P L}^{p}\left(X, \oplus E_{n}\right)$, pour $p$ parcourant $] p_{1}, p_{2}[$, sont stables par dualité au sens suivant : l'injection canonique $\Lambda_{p}: \mathbf{P L}^{q}\left(X, \oplus E_{n}\right) \rightarrow \mathbf{P L}^{p}\left(X, \oplus E_{n}\right)^{\prime} q u i$ à un élément $w \in \mathbf{P L}^{q}\left(X, \oplus E_{n}\right)$ associe la forme linéaire

$$
\begin{aligned}
\mathbf{P L}^{p}\left(X, \oplus E_{n}\right) & \mapsto \mathbb{C} \\
u & \mapsto \sum_{n \geq 0}\left\langle u_{n}, w_{n}\right\rangle_{L^{2}(X)}
\end{aligned}
$$

est un isomorphisme d'espaces de Banach.

En d'autres termes, (35) et (37) impliquent que le dual de $\mathbf{P} \mathbf{L}^{p}\left(X, \oplus E_{n}\right)$ est canoniquement isomorphe à $\mathbf{P L}^{q}\left(X, \oplus E_{n}\right)$ pour tout $\left.p \in\right] p_{1}, p_{2}[$. Les deux théorèmes précédents appellent à quelques remarques :

a) L'inégalité (38) implique l'estimation $\left\|\Lambda_{p}\right\| \leq 1$ mais nous verrons que l'injection canonique $\Lambda_{p}$ n'est généralement pas une isométrie de $\mathbf{P} \mathbf{L}^{p}\left(X, \oplus E_{n}\right)$ sur $\mathbf{P} \mathbf{L}^{q}\left(X, \oplus E_{n}\right)$ (voir $(62)$ ). Cela contraste avec la dualité des espaces de Lebesgue.

b) En pratique, les hypothèses $(36)$ et $(37)$ se réalisent si les densités $\frac{1}{d_{n}} e_{n}$ ont tendance à se concentrer uniformément sur un borélien $A_{n} \subset X$ de mesure finie et strictement positive, c'est-à-dire si l'on peut assimiler la densité de probabilité $\frac{1}{\operatorname{dim}\left(E_{n}\right)} e_{n}$ à une fonction de la forme $x \mapsto \frac{1}{\mu\left(A_{n}\right)} \mathbf{1}_{A_{n}}(x)$. Dans les exemples issus de la physique mathématique qui font intervenir des polynômes orthogonaux, on a très souvent des propriétés de concentration qui forcent $\left\|\sqrt{e_{n}}\right\|_{L^{p}(X)}$ à être équivalent à $n^{\left(a+\frac{b}{p}\right)}$, pour un certain couple $(a, b) \in \mathbb{R}^{2}$, si $p$ parcourt un intervalle donné $] p_{1}, p_{2}[$. Ainsi, (36) sera vérifié. De même (37) sera vérifié si $\left\|\sqrt{e_{n}}\right\|_{L^{p}(X)}$ est équivalent à $n^{a\left(\frac{1}{2}-\frac{1}{p}\right)} \sqrt{\operatorname{dim}\left(E_{n}\right)}$ pour un certain réel $a$.

c) l'hypothèse (35) est très importante dans nos preuves. Bien qu'il ne semble pas facile de l'interpréter, elle sera toujours réalisée dans les cas qui nous concernent. Il est sans doute possible de relaxer cette hypothèse (et peut-être même de la supprimer), mais permettons-nous d'expliquer l'intervention des espaces de Lorentz. D'une part, nos démonstrations utilisent l'interpolation réelle, théorie dans laquelle les espaces de Lorentz jouent un rôle clé. D'autre part, nous verrons des exemples bien concrets, à savoir les fonctions propres qui se concentrent sur une géodésique de la sphère $\mathbb{S}^{d} \subset \mathbb{R}^{d+1}$, où l'hypothèse (35) ne se réalise pas si l'on remplace l'espace de Lorentz $L^{1, \infty}\left(\mathbb{S}^{d}\right)$ par l'espace de Lebesgue $L^{1}\left(\mathbb{S}^{d}\right)$ (voir la remarque 3.7$)$.

2.3. Preuve du théorème 2.1, randomisation avec des matrices aléatoires. - Dans la théorie unidimensionnelle, les inégalités de Kahane-Khintchine, que nous rappelons, sont très importantes : pour tous réels $p>q \geq 1$, il existe une constante $K_{p, q} \geq 1$ telle que, pour tout espace de Banach $B$ et tous éléments $u_{0}, \ldots, u_{N}$ de $B$, les moments des variables aléatoires $\left\|\sum_{n=0}^{N} \varepsilon_{n} u_{n}\right\|_{B}$ sont du même ordre de grandeur au 
sens suivant

$$
\mathbf{E}\left[\left\|\sum_{n=0}^{N} \varepsilon_{n} u_{n}\right\|_{B}^{q}\right]^{\frac{1}{q}} \leq \mathbf{E}\left[\left\|\sum_{n=0}^{N} \varepsilon_{n} u_{n}\right\|_{B}^{p}\right]^{\frac{1}{p}} \leq K_{p, q} \mathbf{E}\left[\left\|\sum_{n=0}^{N} \varepsilon_{n} u_{n}\right\|_{B}^{q}\right]^{\frac{1}{q}} .
$$

D'après Kwapień, il existe une constante universelle $K>0$ telle que l'on a $K_{p, q} \leq K_{p, 1} \leq K \sqrt{p}$ ([36, Part 3.III]).

Fixons maintenant une suite d'entiers non nuls $\left(d_{n}\right)_{n \in \mathbb{N}}$ et notons $\left(\mathcal{E}_{n}\right)_{n}$ une suite de matrices aléatoires indépendantes. On supposera que la matrice aléatoire $\mathcal{E}_{n}$ suit une loi uniforme dans le groupe orthogonal $O_{d_{n}}(\mathbb{R})$ pour tout $n \in \mathbb{N}$. On définit de même $W_{n}$ en remplaçant les groupes orthogonaux $O_{d_{n}}(\mathbb{R})$ par les groupes unitaires $U_{d_{n}}(\mathbb{C})$. Comme nous manipulerons des matrices de tailles différentes, il sera commode de faire le raccourci suivant : à la place de "une suite $\left(b_{n}\right)_{n \in \mathbb{N}}$ de matrices carrées telles que pour tout $n \in \mathbb{N}$ la matrice $b_{n}$ soit de taille $d_{n} \times d_{n}$ à coefficients dans $B$ ", nous écrirons "une suite de matrices carrées $b_{n} \in \mathcal{M}_{d_{n}}(B)$ ". Dans ce contexte, les inégalités de Kahane-Khintchine démontrées par Marcus et Pisier s'énoncent comme suit (voir [39, Page 81, line (2.1) and Page 91, Corollary 2.12]).

Proposition 2.7. - Pour tous réels $p>q \geq 1$, il existe une constante $K_{p, q} \geq 1$ telle que, pour tout espace de Banach $B$, pour tout entier $N \in \mathbb{N}$ et toute suite de matrices carrées $b_{n} \in \mathcal{M}_{d_{n}}(B)$, nous avons

$$
\mathbf{E}\left[\left\|\sum_{n=0}^{N} \operatorname{tr}\left(\mathcal{E}_{n} b_{n}\right)\right\|_{B}^{q}\right]^{\frac{1}{q}} \leq \mathbf{E}\left[\left\|\sum_{n=0}^{N} \operatorname{tr}\left(\mathcal{E}_{n} b_{n}\right)\right\|_{B}^{p}\right]^{\frac{1}{p}} \leq K_{p, q} \mathbf{E}\left[\left\|\sum_{n=0}^{N} \operatorname{tr}\left(\mathcal{E}_{n} b_{n}\right)\right\|_{B}^{q}\right]^{\frac{1}{q}} .
$$

De même que pour les inégalités de Kahane-Khintchine (39), il existe une constante numérique K $\geq 1$ telle que l'on a $K_{p, q} \leq K_{p, 1} \leq K \sqrt{p}$. Des inégalités similaires sont valides pour les matrices aléatoires $W_{n}$ à la place de $\mathcal{E}_{n}$.

La version originale des inégalités (40) utilise les séries aléatoires $\sum d_{n} \operatorname{tr}\left(\mathcal{E}_{n} b_{n}\right)$ qui sont plus adaptées à la théorie des séries de Fourier sur un groupe compact, mais l'on peut bien entendu englober l'entier $d_{n}$ dans la matrice $b_{n}$ (il est d'ailleurs remarquable que ces inégalités ne nécessitent aucune condition de croissance sur les dimensions $d_{n}$ ). Comme les inégalités (40) impliquent les inégalités (39), on se permet d'utiliser la même notation $K_{p, q}$. Les inégalités (40) jouent un rôle clé pour montrer le théorème suivant [39, page 92, Corollary 2.14] où nous forçons l'apparition du terme multiplicatif $\sqrt{d_{n}}$ par cohérence avec la suite.

Théorème 2.8. - Considérons un espace de Banach complexe $B$ et une suite de matrices carrées $b_{n} \in$ $\mathcal{M}_{d_{n}}(B)$. Les propriétés suivantes sont équivalentes

i) la série $\sum_{n} \sqrt{d_{n}} \operatorname{tr}\left(\mathcal{E}_{n} b_{n}\right)$ converge dans $L^{p}(\Omega, B)$ pour un réel $p \in[1,+\infty[$,

ii) la série $\sum_{n} \sqrt{d_{n}} \operatorname{tr}\left(\mathcal{E}_{n} b_{n}\right)$ converge dans $L^{p}(\Omega, B)$ pour tout réel $p \in[1,+\infty[$,

iii) la série $\sum_{n} \sqrt{d_{n}} \operatorname{tr}\left(\mathcal{E}_{n} b_{n}\right)$ converge presque sûrement dans $B$,

iv) la série $\sum_{n} \sqrt{d_{n}} \operatorname{tr}\left(W_{n} b_{n}\right)$ converge dans $L^{p}(\Omega, B)$ pour un réel $p \in[1,+\infty[$,

v) la série $\sum_{n} \sqrt{d_{n}} \operatorname{tr}\left(W_{n} b_{n}\right)$ converge dans $L^{p}(\Omega, B)$ pour tout réel $p \in[1,+\infty[$,

vi) la série $\sum_{n} \sqrt{d_{n}} \operatorname{tr}\left(W_{n} b_{n}\right)$ converge presque sûrement dans $B$.

D'après le théorème de Maurey-Pisier (Théorème 1.6), il faut nécessairement faire une hypothèse supplémentaire si l'on veut ajouter d'autres lois matricielles en plus des matrices aléatoires $\left(\mathcal{E}_{n}\right)$ et $\left(W_{n}\right)$. Le théorème 2.1 explique que l'on peut considérablement préciser le théorème 2.8 dans le cas particulier $B=L^{p}(X)$, avec $p \in[1,+\infty[$. Nous allons exploiter la preuve du théorème 2.8, c'est-à-dire l'utilisation systématique des inégalités (40) et d'un principe de contraction (Théorème 2.16). La partie 2.4 donnera un éclairage sur les propriétés géométriques de l'espace de Banach $L^{p}(X)$ utilisées dans notre argumentation.

Dans le cas $B=\mathbb{C}$, les inégalités (40) donnent un résultat simple et bien connu dans le cas unidimensionnel. Le cas multidimensionnel fait intervenir les normes de Hilbert-Schmidt (30).

Lemme 2.9. - Pour toute suite de matrices $a_{n} \in \mathcal{M}_{d_{n}}(\mathbb{C})$ et pour tout $N \in \mathbb{N}$, on a

$$
\mathbf{E}\left[\left|\sum_{n=0}^{N} \sqrt{d_{n}} \operatorname{tr}\left(\mathcal{E}_{n} a_{n}\right)\right|^{2}\right]=\sum_{n=0}^{N}\left\|a_{n}\right\|_{H S}^{2} .
$$

La même conclusion est valide en remplaçant $\left(\mathcal{E}_{n}\right)_{n \geq 0} \operatorname{par}\left(W_{n}\right)_{n \geq 0}$. 
Preuve. En notant $d P$ la mesure de Haar normalisée du groupe compact $O_{d}(\mathbb{R})$ et en utilisant l'indépendance des matrices aléatoires $\mathcal{E}_{n}$, on a

$$
\begin{aligned}
\mathbf{E}\left[\left|\sum_{n=0}^{N} \sqrt{d_{n}} \operatorname{tr}\left(\mathcal{E}_{n} a_{n}\right)\right|^{2}\right] \\
=\sum_{n_{1}=0}^{N} \sum_{n_{2}=0}^{N} \sqrt{d_{n_{1}}} \sqrt{d_{n_{2}}} \mathbf{E}\left[\operatorname{tr}\left(\mathcal{E}_{n} a_{n}\right) \overline{\operatorname{tr}\left(\mathcal{E}_{n} a_{n}\right)}\right] \\
=\sum_{\substack{0 \leq n_{1} \leq N \\
0 \leq n_{2} \leq N}} \sqrt{d_{n_{1}} d_{n_{2}}}\left(\int_{O_{d_{n_{1}}}(\mathbb{R})} \operatorname{tr}\left(P a_{n_{1}}\right) d P\right)\left(\int_{O_{d_{n_{2}}}(\mathbb{R})} \overline{\operatorname{tr}\left(P a_{n_{2}}\right)} d P\right)+\sum_{n=0}^{N} d_{n} \int_{O_{d_{n}}(\mathbb{R})}\left|\operatorname{tr}\left(P a_{n}\right)\right|^{2} d P .
\end{aligned}
$$

Il nous suffit de prouver pour tout entier $d \in \mathbb{N}^{\star}$ et pour toute matrice $A \in \mathcal{M}_{d}(\mathbb{C})$ :

$$
\int_{O_{d}(\mathbb{R})} \operatorname{tr}(P A) d P=0 \text { et } \int_{O_{d}(\mathbb{R})}|\operatorname{tr}(P A)|^{2} d P=\frac{\operatorname{tr}\left({ }^{t} \bar{A} A\right)}{d} .
$$

Le cas $d=1$ étant trivial, on suppose $d \geq 2$. La nullité de la première intégrale est claire par le changement de variables $P \mapsto-P$. Pour la seconde intégrale, il suffit de traiter le cas où tous les coefficients de $A$ sont réels. En effet, le cas complexe découle du cas réel en écrivant $A=A_{x}+i A_{y}$ avec $A_{x}, A_{y} \in \mathcal{M}_{d}(\mathbb{R})$. En notant la matrice symétrique $|A|=\sqrt{{ }^{t} A A}$, on peut factoriser $A=P|A|=P Q D Q^{-1}$ avec $(P, Q) \in O_{d}(\mathbb{R})^{2}$ et $D$ matrice diagonale dont les valeurs propres $\mu_{1}, \ldots, \mu_{d}$ sont les valeurs singulières de $A$. Les propriétés d'invariance de la trace et de la mesure de Haar $d P$ simplifient l'intégrale

$$
\begin{aligned}
\int_{O_{d}(\mathbb{R})} \operatorname{tr}(P A)^{2} d P & =\int_{O_{d}(\mathbb{R})} \operatorname{tr}(P D)^{2} d P \\
& =\int_{O_{d}(\mathbb{R})}\left(p_{11} \mu_{1}+\cdots+p_{d d} \mu_{d}\right)^{2} d P \\
& =\sum_{i, j=1}^{d} \mu_{i} \mu_{j} \int_{O_{d}(\mathbb{R})} p_{i i} p_{j j} d P .
\end{aligned}
$$

En effectuant les changements de coordonnées $P \mapsto E_{i j} P$ et $P \mapsto P E_{i j}$ où $E_{i j}$ est la matrice orthogonale associée à la permutation qui transpose $i$ et $j$ ainsi que les $d$ changements de coordonnées $P \mapsto \Delta_{k} P$ où $\Delta_{k}$ est la matrice diagonale $\operatorname{Diag}(1, \ldots, 1, \underbrace{-1}_{k}, 1, \ldots, 1)$, on obtient

$$
\begin{array}{rlrl}
\forall i \neq j & \int_{O_{d}(\mathbb{R})} p_{i i} p_{j j} d P & =0 \\
\forall i, j \quad \int_{O_{d}(\mathbb{R})} p_{i j}^{2} d P & =\int_{O_{d}(\mathbb{R})} p_{11}^{2} d P .
\end{array}
$$

Par moyenne, la dernière intégrale vaut

$$
\frac{1}{d^{2}} \int_{O_{d}(\mathbb{R})} \sum_{i=1}^{d} \sum_{j=1}^{d} p_{i j}^{2} d P=\frac{1}{d^{2}} \int_{O_{d}(\mathbb{R})} \operatorname{tr}\left({ }^{t} P P\right) d P=\frac{1}{d^{2}} \int_{O_{d}(\mathbb{R})} \operatorname{tr}\left(I_{d}\right) d P=\frac{1}{d} .
$$

Cela prouve $(41) \operatorname{car} \operatorname{tr}\left({ }^{t} A A\right)=\mu_{1}^{2}+\cdots+\mu_{d}^{2}$.

Nous avons à présent tous les moyens pour aborder la randomisation de matrices aléatoires plus générales.

Proposition 2.10. - Fixons $q \in\left[2,+\infty\left[\right.\right.$ et considérons une suite de matrices aléatoires $M_{n}: \Omega \rightarrow$ $\mathcal{M}_{d_{n}}(\mathbb{R})$ indépendantes, orthogonalement invariantes et vérifiant l'hypothèse de bornitude des moments

$$
\sup _{n \in \mathbb{N}} \mathbf{E}\left[\left\|M_{n}\right\|_{o p}^{q}\right]<+\infty \text {. }
$$


Il existe une constante universelle $K \geq 1$ telle que, pour toute suite $a_{n} \in \mathcal{M}_{d_{n}}(\mathbb{C})$, nous avons

$$
\forall N \in \mathbb{N} \quad \mathbf{E}\left[\left|\sum_{n=0}^{N} \sqrt{d_{n}} \operatorname{tr}\left(M_{n} a_{n}\right)\right|^{q}\right]^{\frac{1}{q}} \leq K \sqrt{q}\left(\sup _{0 \leq n \leq N} \mathbf{E}\left[\left\|M_{n}\right\|_{o p}^{q}\right]\right)^{\frac{1}{q}} \sqrt{\sum_{n=0}^{N}\left\|a_{n}\right\|_{H S}^{2} .}
$$

La même conclusion est valide en supposant que chaque matrice aléatoire $M_{n}$ est à valeurs dans $\mathcal{M}_{d_{n}}(\mathbb{C})$ et est unitairement invariante.

Preuve. Fixons $\mathcal{E}_{0}, \ldots, \mathcal{E}_{N}$ des matrices aléatoires indépendantes (et indépendantes des matrices aléatoires $M_{n}$ ) qui suivent des lois uniformes dans les groupes orthogonaux de tailles respectives $d_{0}, \ldots, d_{N}$. Utilisant la définition 1.7 et les inégalités de Kahane-Khintchine-Marcus-Pisier (40) et (41), on peut écrire

$$
\begin{aligned}
& \mathbf{E}\left[\left|\sum_{n=0}^{N} \sqrt{d_{n}} \operatorname{tr}\left(M_{n} a_{n}\right)\right|^{q}\right]=\mathbf{E}_{\omega^{\prime}} \mathbf{E}_{\omega}\left[\left|\sum_{n=0}^{N} \sqrt{d_{n}} \operatorname{tr}\left(\mathcal{E}_{n}\left(\omega^{\prime}\right) M_{n}(\omega) a_{n}\right)\right|_{q}^{q}\right] \\
&\left.=\left.\mathbf{E}_{\omega} \mathbf{E}_{\omega^{\prime}}\left[\left|\sum_{n=0}^{N} \sqrt{d_{n}} \operatorname{tr}\left(\mathcal{E}_{n}\left(\omega^{\prime}\right) M_{n}(\omega) a_{n}\right)\right|^{q}\right]\right|^{\frac{q}{q}}\right] \\
& \leq K^{q} q^{\frac{q}{2}} \mathbf{E}_{\omega}\left[\mathbf{E}_{\omega^{\prime}}\left[\left|\sum_{n=0}^{N} \sqrt{d_{n}} \operatorname{tr}\left(\mathcal{E}_{n}\left(\omega^{\prime}\right) M_{n}(\omega) a_{n}\right)\right|^{\frac{q}{2}}\right]\right. \\
& \leq K^{q} q^{\frac{q}{2}} \mathbf{E}_{\omega}\left[\left(\sum_{n=0}^{N}\left\|M_{n}(\omega) a_{n}\right\|_{H S}^{2}\right)^{\frac{q}{2}}\right] . \\
& \leq K^{q} q^{\frac{q}{2}} \mathbf{E}_{\omega}\left[\left(\sum_{n=0}^{N}\left\|M_{n}(\omega)\right\|_{o p}^{2}\left\|a_{n}\right\|_{H S}^{2}\right)^{2}\right] .
\end{aligned}
$$

L'inégalité triangulaire dans $L^{\frac{q}{2}}(\Omega)$ nous amène alors à la conclusion

$$
\begin{aligned}
\mathbf{E}\left[\left|\sum_{n=0}^{N} \sqrt{d_{n}} \operatorname{tr}\left(M_{n} a_{n}\right)\right|^{q}\right] & \leq K^{q} q^{\frac{q}{2}}\left(\sum_{n=0}^{N}\left\|a_{n}\right\|_{H S}^{2} \mathbf{E}\left[\left\|M_{n}\right\|_{o p}^{q}\right]^{\frac{2}{q}}\right)^{\frac{q}{2}} \\
& \leq K^{q} q^{\frac{q}{2}}\left(\sup _{0 \leq n \leq N} \mathbf{E}\left[\left\|M_{n}\right\|_{o p}^{q}\right]\right)\left(\sum_{n=0}^{N}\left\|a_{n}\right\|_{H S}^{2}\right)^{\frac{q}{2}} .
\end{aligned}
$$

Remarque 2.11. - Dans le cas particulier $d_{n}=1$, c'est-à-dire si les matrices aléatoires $M_{n}: \Omega \rightarrow \mathbb{R}$ sont en fait des variables aléatoires symétriques, l'inégalité (42) s'écrit pour tout réel $q \in[2,+\infty[$ et tous réels $a_{0}, \ldots, a_{N}$ :

$$
\mathbf{E}\left[\left|\sum_{n=0}^{N} M_{n} a_{n}\right|^{q}\right]^{\frac{1}{q}} \leq K \sqrt{q}\left(\sup _{0 \leq n \leq N} \mathbf{E}\left[\left|M_{n}\right|^{q}\right]\right)^{\frac{1}{q}} \sqrt{\sum_{n=0}^{N}\left|a_{n}\right|^{2}} .
$$

Du fait que la dimension considérée est 1 , le principe de symétrisation montrerait que l'inégalité précédente est aussi vraie si les variables aléatoires $M_{n}$ sont seulement centrées (voir la preuve du lemme B.1).

Voici deux lemmes spécifiques à $L^{p}(X)$.

Lemme 2.12. - Pour tous réels $p$ et $q$ appartenant à $\left[1,+\infty\left[\right.\right.$ et pour toute suite de matrices $b_{n} \in$ $\mathcal{M}_{d_{n}}\left(L^{p}(X)\right)$, on a

$$
\mathbf{E}\left[\left\|\sum_{n=0}^{N} \sqrt{d_{n}} \operatorname{tr}\left(\mathcal{E}_{n} b_{n}(x)\right)\right\|_{L_{x}^{p}(X)}^{q}\right]^{\frac{1}{q}} \simeq\left\|\sqrt{\sum_{n=0}^{N}\left\|b_{n}(x)\right\|_{H S}^{2}}\right\|_{L_{x}^{p}(X)} .
$$

La même conclusion est valide en remplaçant $\left(\mathcal{E}_{n}\right)_{n \geq 0} \operatorname{par}\left(W_{n}\right)_{n \geq 0}$. 
Preuve. D'après les inégalités de Kahane-Khintchine-Marcus-Pisier (40), il suffit de traiter le cas $q=p$. Le théorème de Fubini donne alors

$$
\mathbf{E}\left[\left\|\sum_{n=0}^{N} \sqrt{d_{n}} \operatorname{tr}\left(\mathcal{E}_{n} b_{n}(x)\right)\right\|_{L_{x}^{p}(X)}^{p}\right]=\int_{X} \mathbf{E}\left[\left|\sum_{n=0}^{N} \sqrt{d_{n}} \operatorname{tr}\left(\mathcal{E}_{n} b_{n}(x)\right)\right|^{p}\right] d \mu(x) .
$$

De nouveau, les inégalités de Kahane-Khintchine-Marcus-Pisier sur $\mathbb{C}$ montrent que l'intégrale précédente est équivalente à la suivante

$$
\int_{X} \mathbf{E}\left[\left|\sum_{n=0}^{N} \sqrt{d_{n}} \operatorname{tr}\left(\mathcal{E}_{n} b_{n}(x)\right)\right|^{2}\right]^{\frac{p}{2}} d \mu(x)
$$

L'égalité (41) achève la preuve.

Lemme 2.13. - Considérons un réel $p \in\left[1,+\infty\left[\right.\right.$, un entier $N \in \mathbb{N}$, des matrices aléatoires $M_{0}: \Omega \rightarrow$ $\mathcal{M}_{d_{0}}(\mathbb{C}), \ldots, M_{N}: \Omega \rightarrow \mathcal{M}_{d_{N}}(\mathbb{C})$ et des matrices $b_{0} \in \mathcal{M}_{d_{0}}\left(L^{p}(X)\right), \ldots, b_{N} \in \mathcal{M}_{d_{N}}\left(L^{p}(X)\right)$. Alors on a l'inégalité :

$$
\forall q \in\left[p,+\infty\left[\cup\{+\infty\} \quad \mathbf{E}_{\omega}\left[\left\|\sum_{n=0}^{N} \sqrt{d_{n}} \operatorname{tr}\left(M_{n}(\omega) b_{n}\right)\right\|_{L^{p}(X)}^{q}\right] \leq \| \mathbf{E}_{\omega}\left[\left|\sum_{n=0}^{N} \sqrt{d_{n}} \operatorname{tr}\left(M_{n}(\omega) b_{n}\right)\right|^{q}\left\|_{L^{p}(X)}^{\frac{1}{q}}\right\|\right.\right.\right.
$$

Preuve. Il s'agit de remarquer la continuité de l'injection canonique $L^{p}\left(X, L^{q}(\Omega)\right) \rightarrow L^{q}\left(\Omega, L^{p}(X)\right)$ par interpolation entre $q=p$ et $q=+\infty$.

À partir de ce point, nous n'aurons besoin d'aucune autre propriété spécifique de l'espace de Banach $L^{p}(X)$. On obtient facilement le résultat suivant.

Corollaire 2.14. - Considérons $p \in\left[1,+\infty\left[\right.\right.$ et une suite de matrices aléatoires $M_{n}: \Omega \rightarrow \mathcal{M}_{d_{n}}(\mathbb{R})$ indépendantes, orthogonalement invariantes et vérifiant

$$
\sup _{n \in \mathbb{N}} \mathbf{E}\left[\left\|M_{n}\right\|_{o p}^{\max (2, p)}\right]<+\infty .
$$

Il existe une constante universelle $K \geq 1$ telle que, pour toute suite de matrices $b_{n} \in \mathcal{M}_{d_{n}}\left(L^{p}(X)\right)$ et tout entier $N \in \mathbb{N}$, on a

$$
\begin{aligned}
& \left\|\sum_{n=0}^{N} \sqrt{d_{n}} \operatorname{tr}\left(M_{n} b_{n}\right)\right\|_{L^{\max (2, p)\left(\Omega, L^{p}(X)\right)}} \\
& \quad \leq K \sqrt{p}\left(\sup _{n \in \mathbb{N}} \mathbf{E}\left[\left\|M_{n}\right\|_{o p}^{\max (2, p)}\right]\right)^{\frac{1}{\max (2, p)}} \times\left[\int_{X}\left(\sum_{n=0}^{N}\left\|b_{n}(x)\right\|_{H S}^{2}\right)^{\frac{p}{2}} d \mu(x)\right]^{\frac{1}{p}} .
\end{aligned}
$$

Par conséquent, si $x \mapsto \sum_{n \geq 0}\left\|b_{n}(x)\right\|_{H S}^{2}$ appartient à $L^{p / 2}(X)$, alors la série aléatoire $\sum \sqrt{d_{n}} \operatorname{tr}\left(M_{n} b_{n}\right)$ converge dans $L^{\max (2, p)}\left(\Omega, L^{p}(X)\right)$ et presque sûrement dans $L^{p}(X)$.

La même conclusion est valide en supposant que chaque matrice aléatoire $M_{n}$ est à valeurs dans $\mathcal{M}_{d_{n}}(\mathbb{C})$ et est unitairement invariante.

Preuve. L'inégalité (44) découle d'une combinaison du lemme 2.13, l'inégalité (42) avec $q=\max (2, p)$ et de l'inégalité $\sqrt{\max (2, p)} \leq \sqrt{2 p}$.

Pour obtenir la convergence dans $L^{\max (2, p)}\left(\Omega, L^{p}(X)\right)$ de la série aléatoire $\sum \sqrt{d_{n}} \operatorname{tr}\left(M_{n} b_{n}\right)$, il suffit d'appliquer (44) à ses paquets de Cauchy. Ensuite, la convergence dans $L^{\max (2, p)}\left(\Omega, L^{p}(X)\right)$ implique la convergence en probabilité. Puisque les termes de la série aléatoire $\sum \sqrt{d_{n}} \operatorname{tr}\left(M_{n} b_{n}\right)$ sont indépendants et sont à valeurs dans le sous-espace séparable de $L^{p}(X)$ engendré par les coefficients des matrices $b_{n}$, on en déduit sa convergence presque sûre (voir [35, Theorem 6.1] ou [36, Théorème II.3]). 
Remarque 2.15. - On peut reformuler l'inégalité (44) à l'aide de (43)

$$
\begin{aligned}
& \left\|\sum_{n=0}^{N} \sqrt{d_{n}} \operatorname{tr}\left(M_{n} b_{n}\right)\right\|_{L^{\max (2, p)\left(\Omega, L^{p}(X)\right)}} \\
& \quad \leq C(p)\left(\sup _{n \in \mathbb{N}} \mathbf{E}\left[\left\|M_{n}\right\|_{o p}^{\max (2, p)}\right]\right)^{\frac{1}{\max (2, p)}}\left\|\sum_{n=0}^{N} \sqrt{d_{n}} \operatorname{tr}\left(\mathcal{E}_{n} b_{n}\right)\right\|_{L^{\max (2, p)}\left(\Omega, L^{p}(X)\right)} .
\end{aligned}
$$

Cette inégalité a la même forme que celle qui apparaît à la fin de la démonstration de [41, page 69]. Il est donc légitime de croire qu'une démonstration avec un théorème de factorisation est possible.

Rappelons maintenant un principe de contraction pour les variables $\mathcal{E}_{n}$ et $W_{n}$ obtenu par Marcus et Pisier.

Théorème 2.16. - Fixons un espace de Banach complexe B, un réel $q \in[1,+\infty[$, une suite de matrices $b_{n} \in \mathcal{M}_{d_{n}}(B)$ et une suite de matrices aléatoires $M_{n}: \Omega \rightarrow \mathcal{M}_{d_{n}}(\mathbb{R})$ indépendantes et orthogonalement invariantes. Alors on a

$$
\left(\inf _{0 \leq n \leq N} \sigma\left(\mathbf{E}\left[\left|M_{n}\right|\right]\right)\right) \mathbf{E}\left[\left\|\sum_{n=0}^{N} \sqrt{d_{n}} \operatorname{tr}\left(\mathcal{E}_{n} b_{n}\right)\right\|_{B}^{q}\right]^{\frac{1}{q}} \leq \mathbf{E}\left[\left\|\sum_{n=0}^{N} \sqrt{d_{n}} \operatorname{tr}\left(M_{n} b_{n}\right)\right\|_{B}^{q}\right]^{\frac{1}{q}}
$$

Une inégalité similaire est valide en remplaçant $\mathcal{E}_{n}$ par $W_{n}, \mathcal{M}_{d_{n}}(\mathbb{R})$ par $\mathcal{M}_{d_{n}}(\mathbb{C})$ et l'invariance orthogonale par l'invariance unitaire.

Preuve. On consultera [39, Page 82, Proposition 2.1, line (2.7)] avec le terme $\left\|\mathbf{E}\left[\left|M_{n}\right|\right]^{-1}\right\|_{o p}^{-1}=\sigma\left(\mathbf{E}\left[\left|M_{n}\right|\right]\right)$.

Le résultat précédent semble suggérer qu'une minoration uniforme de la forme $\inf _{n \in \mathbb{N}} \sigma\left(\mathbf{E}\left[\left|M_{n}\right|\right]\right)>0$ et la convergence presque sûre de la série aléatoire $\sum \sqrt{d_{n}} \operatorname{tr}\left(M_{n} b_{n}\right)$ impliquent celle de $\sum \sqrt{d_{n}} \operatorname{tr}\left(\mathcal{E}_{n} b_{n}\right)$. En fait, il est aisé de construire un contre-exemple dans le cas plus simple, à savoir $d_{n}=1$ pour tout $n \in \mathbb{N}$ et $B=\mathbb{R}$. Pour cela, considérons une suite indépendante de variables aléatoires symétriques $X_{n}: \Omega \rightarrow \mathbb{R}$, avec $n \geq 1$, telle que la loi de $X_{n}$ est

$$
\frac{\delta_{-n^{2}}}{2 n^{2}}+\left(1-\frac{1}{n^{2}}\right) \delta_{0}+\frac{\delta_{n^{2}}}{2 n^{2}}
$$

On a une concentration en 0 au sens suivant : il existe une constante $C>1$ telle que

$$
\mathbf{P}\left[X_{n}=0\right]=1-\frac{1}{n^{2}}, \quad \mathbf{P}\left[\left|X_{n}\right| \geq 1\right]=\frac{1}{n^{2}}, \quad \mathbf{E}\left[\left|X_{n}\right|\right] \in\left[\frac{1}{C}, C\right] .
$$

Le lemme de Borel-Cantelli assure que pour presque tout $\omega \in \Omega$, la suite $\left(X_{n}(\omega)\right)$ stationne en 0 , donc la série aléatoire $\sum X_{n}$ converge presque sûrement tandis que la série aléatoire $\sum \varepsilon_{n}$ diverge toujours. Cet exemple élémentaire montre qu'il faut nécessairement imposer des conditions supplémentaires sur les matrices aléatoires $M_{n}$ pour obtenir la convergence presque sûre de la série aléatoire $\sum \operatorname{tr} \sqrt{d_{n}} \operatorname{tr}\left(\mathcal{E}_{n} b_{n}\right)$ (voir par exemple [31, Part 5] et [30, Theorem 5.2] dans le cas unidimensionnel). Un défaut du contre-exemple précédent est l'explosion des moments d'ordre strictement plus grand que 1 . Cela nous mène à la proposition suivante qui nous a été inspirée par la preuve de [39, Page 55, Lemma 1.2]. Est examinée dans cette référence la situation pour un espace de Banach de la forme $\mathcal{C}^{0}(K)$, où $K$ est compact d'un groupe localement compact, à la place de $L^{p}(X)$. L'idée de la preuve se résume simplement : nous allons majorer les moments d'ordre 2 des sommes partielles de la série $\sum \sqrt{d_{n}} \operatorname{tr}\left(M_{n} b_{n}\right)$ par leurs moments d'ordre 1 (ce qui inverse l'ordre naturel), puis la bornitude presque sûre impliquera que les moments d'ordre 1 et 2 sont uniformément bornés. Par comparaison avec les variables aléatoires $\mathcal{E}_{n}$, nous obtiendrons la conclusion.

Proposition 2.17. - Fixons $p \in\left[1,+\infty\left[\right.\right.$ et une suite de matrices aléatoires $M_{n}: \Omega \rightarrow \mathcal{M}_{d_{n}}(\mathbb{R})$ indépendantes, orthogonalement invariantes et vérifiant

$$
\inf _{n \in \mathbb{N}} \sigma\left(\mathbf{E}\left[\left|M_{n}\right|\right]\right)>0 \quad \text { et } \quad \sup _{n \in \mathbb{N}} \mathbf{E}\left[\left\|M_{n}\right\|_{o p}^{\max (2, p)}\right]<+\infty
$$


Pour toute suite de matrices $b_{n} \in \mathcal{M}_{d_{n}}\left(L^{p}(X)\right)$, si la série aléatoire $\sum \sqrt{d_{n}} \operatorname{tr}\left(M_{n} b_{n}\right)$ est bornée en probabilité dans $L^{p}(X)$, c'est-à-dire si l'on a

$$
\lim _{t \rightarrow+\infty} \sup _{N \in \mathbb{N}} \mathbf{P}\left[\left\|\sum_{n=0}^{N} \sqrt{d_{n}} \operatorname{tr}\left(M_{n} b_{n}\right)\right\|_{L^{p}(X)}>t\right]=0,
$$

alors

$$
\sqrt{\sum_{n \in \mathbb{N}}\left\|b_{n}(x)\right\|_{H S}^{2}} \in L_{x}^{p}(X) .
$$

De nouveau, la même conclusion est valide en supposant que chaque matrice aléatoire $M_{n}$ est à valeurs dans $\mathcal{M}_{d_{n}}(\mathbb{C})$ et est unitairement invariante.

Preuve. Posons pour tout entier $N \in \mathbb{N}$ la somme partielle

$$
S_{N}=\sum_{n=0}^{N} \sqrt{d_{n}} \operatorname{tr}\left(M_{n} b_{n}\right)
$$

En outre, on considère $c>1$ de sorte que l'on a pour tout $n \in \mathbb{N}$

$$
\frac{1}{c} \leq \sigma\left(\mathbf{E}\left[\left|M_{n}\right|\right]\right) \quad \text { et } \quad \mathbf{E}\left[\left\|M_{n}\right\|_{o p}^{\max (2, p)}\right] \leq c .
$$

Le principe de contraction (théorème 2.16) et les inégalités (43) montrent qu'il existe une constante $C(p, c)>$ 1 telle que

$$
\begin{aligned}
&{ }_{c}^{1} \mathbf{E}\left[\left\|\sum_{n=0}^{N} \sqrt{d_{n}} \operatorname{tr}\left(\mathcal{E}_{n} b_{n}(x)\right)\right\|_{L_{x}^{p}(X)}\right] \leq \mathbf{E}\left[\left\|S_{N}\right\|_{L^{p}(X)}\right] \\
& \frac{1}{C(p, c)}\left\|\sqrt{\sum_{n=0}^{N}\left\|b_{n}(x)\right\|_{H S}^{2}}\right\|_{L_{x}^{p}(X)} \leq \mathbf{E}\left[\left\|S_{N}\right\|_{L^{p}(X)}\right] .
\end{aligned}
$$

Majorons maintenant les moments d'ordre 2. En invoquant (44) et quitte à augmenter $C(p, c)$, on a

$$
\begin{aligned}
\mathbf{E}\left[\left\|S_{N}\right\|_{L^{p}(X)}^{2}\right]^{\frac{1}{2}} & \leq \mathbf{E}\left[\left\|S_{N}\right\|_{L^{p}(X)}^{\max (2, p)}\right]^{\frac{1}{\max (2, p)}} \\
& \leq C(p, c)\left\|\sqrt{\sum_{n=0}^{N}\left\|b_{n}(x)\right\|_{H S}^{2}}\right\|_{L_{x}^{p}(X)} \\
& \leq C(p, c)^{2} \mathbf{E}\left[\left\|S_{N}\right\|_{L^{p}(X)}\right] .
\end{aligned}
$$

Il s'agit de l'inégalité d'inversion des moments que nous cherchions. Utilisons l'inégalité de Paley-Zygmund [32, Page 8, Inequality II] pour obtenir

$$
\mathbf{P}\left[\left\|S_{N}\right\|_{L^{p}(X)} \geq \frac{1}{2} \mathbf{E}\left[\left\|S_{N}\right\|_{L^{p}(X)}\right]\right] \geq \frac{\mathbf{E}\left[\left\|S_{N}\right\|_{L^{p}(X)}\right]^{2}}{4 \mathbf{E}\left[\left\|S_{N}\right\|_{L^{p}(X)}^{2}\right]} \geq \frac{1}{4 C(p, c)^{4}} .
$$

D'après (46), il existe un réel $A>0$ tel que

$$
\forall N \in \mathbb{N} \quad \frac{1}{4 C(p, c)^{4}}>\mathbf{P}\left[\left\|S_{N}\right\|_{L^{p}(X)} \geq A\right] .
$$

Or (48) force chaque moment $\mathbf{E}\left[\left\|S_{N}\right\|_{L^{p}(X)}\right]$ à être majoré par $2 A$. La conclusion découle en examinant (47). 
La preuve du théorème 2.1 est obtenue en combinant tous les arguments précédents. En effet, on vérifie les implications i) $\Rightarrow$ iii) et iv) $\Rightarrow$ i) grâce au corollaire 2.14 et à la proposition 2.17. L'implication iii) $\Rightarrow$ ii) a été vue au cours de la preuve du corollaire 2.14. Quant à l'implication ii) $\Rightarrow$ iv), elle est vraie en toute généralité.

2.4. Randomisation dans un treillis de Banach de cotype fini et preuve du théorème 1.9. On explique comment les arguments développés dans la partie 2.3 s'étendent pour les treillis de Banach (pour cette notion, on se réfère au livre [37]). Par souci de simplicité, on ne considérera que des espaces de Banach sur le corps des réels. Il est en fait possible de complexifier un treillis de Banach afin de déduire des résultats complexes à partir de résultats réels (voir $[\mathbf{3 7}$, page 43$]$ ). On notera $\leq, \vee$ et $|\cdot|$ respectivement la relation d'ordre, la borne supérieure et la valeur absolue. Pour tout $N \in \mathbb{N}^{\star}$, on notera aussi $\overline{\mathcal{H}_{N}}$ l'espace vectoriel réel des fonctions continues et 1-homogènes sur $\mathbb{R}^{N}$ ainsi que les projections sur les coordonnées :

$$
\phi_{i}:\left(t_{1}, \ldots, t_{N}\right) \in \mathbb{R}^{N} \mapsto t_{i} \in \mathbb{R} .
$$

On notera que $\overline{\mathcal{H}_{N}}$ est un treillis. Le théorème suivant assure l'existence d'un calcul fonctionnel dans un treillis de Banach basé sur $\overline{\mathcal{H}_{n}}$ (voir [37, Theorem 1d1]).

Théorème 2.18. - Soit B un treillis de Banach et fixons $f_{1}, \ldots, f_{N}$ des éléments de B. Il existe une unique application linéaire

$$
\begin{aligned}
\tau: \overline{\mathcal{H}_{N}} & \rightarrow B \\
F & \mapsto F\left(f_{1}, \ldots, f_{N}\right)
\end{aligned}
$$

qui vérifie les deux propriétés suivantes :

i) $\phi_{i}\left(f_{1}, \ldots, f_{N}\right)=f_{i}$ pour tout entier $i \in[1, N]$,

ii) $\tau$ est un morphisme de treillis (i.e. conserve positivité, borne supérieure, borne inférieure et valeur absolue).

Dans ce cas, en notant $f=\left|f_{1}\right| \vee \cdots \vee\left|f_{N}\right|$, on a l'estimation de continuité

$$
\left\|F\left(f_{1}, \ldots, f_{N}\right)\right\|_{B} \leq\|f\|_{B} \sup _{\|t\|_{\infty} \leq 1}\left|F\left(t_{1}, \ldots, t_{N}\right)\right| .
$$

Rappelons maintenant la définition suivante.

Définition 2.19. - Un treillis de Banach B est q-concave, avec $q \in\left[1,+\infty\left[\right.\right.$, s'il existe un réel $M_{(q)}(B)>0$ tel que l'on a l'inégalité suivante pour tout entier $N \in \mathbb{N}^{\star}$ et tout élément $\left(f_{1}, \ldots, f_{N}\right) \in B^{N}$ :

$$
\left(\sum_{i=1}^{N}\left\|f_{i}\right\|_{B}^{q}\right)^{\frac{1}{q}} \leq M_{(q)}(B)\left\|\left(\sum_{i=1}^{N}\left|f_{i}\right|^{q}\right)^{\frac{1}{q}}\right\|_{B}
$$

où le terme $\left(\sum_{i=1}^{N}\left|f_{i}\right|^{q}\right)^{\frac{1}{q}} \in B$ est défini par calcul fonctionnel.

La notion de $q$-concavité est reliée à celle de cotype comme le montre le résultat suivant [37, Proposition 1f3, Corollary 1f9].

Proposition 2.20. - Soient $B$ un treillis de Banach et un réel $q \in[2,+\infty[$, on a

i) si $B$ est $q$-concave, alors $B$ est de cotype $q$,

ii) si $B$ est de cotype $q \in[2,+\infty[$ alors $B$ est $(q+\varepsilon)$-concave pour tout $\varepsilon \in] 0,+\infty[$.

Par conséquent, on a

$$
\inf \{q \geq 2, \quad B \text { est } q \text {-concave }\}=\inf \{q \geq 2, \quad B \text { est de cotype } q\} .
$$

On vérifie que $L^{p}(\mathbb{R})$ est $q$-concave si $1 \leq p \leq q\left(\right.$ il s'agit d'interpoler l'injection canonique $L^{p}\left(\mathbb{R}, \ell^{q}(\mathbb{N})\right) \rightarrow$ $\ell^{q}\left(\mathbb{N}, L^{p}(\mathbb{R})\right)$ entre $q=p$ et $\left.q=\infty\right)$ et la borne inférieure (51) vaut $\max (2, p)$. Un autre exemple intéressant est fourni par les espaces de Lorentz $L^{p, 1}(\mathbb{R})$ (voir les calculs exacts dans $[\mathbf{1 6}]$ ).

Pour ce qui nous concerne, la proposition précédente est le point crucial qui permet de s'émanciper de la randomisation unidimensionnelle. En effet la définition de la $q$-concavité ne fait pas intervenir de variables aléatoires de Bernoulli (contrairement à la définition du cotype (19)). Nous disposons maintenant du 
vocabulaire adéquat pour énoncer une version multidimensionnelle et quantitative du théorème de MaureyPisier dans la catégorie des treillis de Banach. De façon précise, le théorème ci-dessous généralise le théorème 2.1 et implique le théorème 1.9 .

Théorème 2.21. - Considérons un treillis de Banach $B$-concave avec $q \in[2,+\infty[$, une suite d'entiers non nuls $\left(d_{n}\right)_{n \in \mathbb{N}}$, une suite de matrices $b_{n} \in \mathcal{M}_{d_{n}}(B)$, et une suite de matrices aléatoires $M_{n}: \Omega \rightarrow \mathcal{M}_{d_{n}}(\mathbb{R})$ indépendantes, orthogonalement invariantes et vérifiant

$$
\inf _{n \in \mathbb{N}} \sigma\left(\mathbf{E}\left[\left|M_{n}\right|\right]\right)>0 \quad \text { et } \sup _{n \in \mathbb{N}} \mathbf{E}\left[\left\|M_{n}\right\|_{o p}^{q}\right]<+\infty
$$

Alors on a l'équivalence des propriétés suivantes :

i) les normes $\left\|\sqrt{\sum_{n=0}^{N}\left\|b_{n}\right\|_{H S}^{2}}\right\|_{B}$ sont majorées indépendamment de $N \in \mathbb{N}$,

ii) la série aléatoire $\sum \sqrt{d_{n}} \operatorname{tr}\left(M_{n} b_{n}\right)$ converge presque sûrement dans $B$,

iii) la série aléatoire $\sum \sqrt{d_{n}} \operatorname{tr}\left(M_{n} b_{n}\right)$ converge dans $L^{q}(\Omega, B)$,

iv) la série aléatoire $\sum \sqrt{d_{n}} \operatorname{tr}\left(M_{n} b_{n}\right)$ est bornée en probabilité dans $B$.

Pour prouver le théorème 2.21, nous aurons besoin du lemme suivant. L'inégalité (53) ci-dessous est seulement une version généralisée de (50).

Lemme 2.22. - Considérons des variables aléatoires $X_{1}, \ldots, X_{N}$ appartenant à $L^{1}(\Omega)$ et des éléments $f_{1}, \ldots, f_{N}$ d'un treillis de Banach $B$. Alors on a "l'inégalité triangulaire" :

$$
\left\|\mathbf{E}_{\omega}\left[\left|\sum_{n=1}^{N} X_{n}(\omega) f_{n}\right|\right]\right\|_{B} \leq \mathbf{E}_{\omega}\left[\left\|\sum_{n=1}^{N} X_{n}(\omega) f_{n}\right\|_{B}\right] .
$$

S'il existe un réel $q \in\left[1,+\infty\left[\right.\right.$ tel que $B$ soit q-concave et que les variables aléatoires $X_{1}, \ldots, X_{N}$ appartiennent $\grave{a} L^{q}(\Omega)$, alors on a aussi

$$
\mathbf{E}_{\omega}\left[\left\|\sum_{n=1}^{N} X_{n}(\omega) f_{n}\right\|_{B}^{q}\right]^{\frac{1}{q}} \leq M_{(q)}(B)\left\|\mathbf{E}_{\omega}\left[\left|\sum_{n=1}^{N} X_{n}(\omega) f_{n}\right|^{q}\right]^{\frac{1}{q}}\right\|_{B},
$$

où les espérances dans le membre gauche de (52) et dans le membre droit de (53) sont définies par calcul fonctionnel sur les $N$ variables $f_{1}, \ldots, f_{N}$.

Preuve. On commence par (53). Pour tout entier $n \in[1, N]$, on note $\left(X_{n, k}\right)_{k \in \mathbb{N}}$ une suite de variables aléatoires qui prend un nombre fini de valeurs et qui converge dans $L^{q}(\Omega)$ vers $X_{n}$. Pour tout $k \in \mathbb{N}$, il existe donc une partition finie

$$
\Omega=\bigsqcup_{\ell=1}^{L} \Omega_{k, \ell}
$$

en parties mesurables telle que chaque variable aléatoire $X_{n, k}$ prend une valeur fixe, disons $x_{n, k, \ell}$, sur $\Omega_{k, \ell}$. La $q$-concavité de $B$ nous donne alors

$$
\begin{aligned}
\mathbf{E}_{\omega}\left[\left\|\sum_{n=1}^{N} X_{n, k}(\omega) f_{n}\right\|_{B}^{q}\right]^{\frac{1}{q}} & =\left(\sum_{\ell=1}^{L} \mathbf{P}\left(\Omega_{k, \ell}\right)\left\|\sum_{n=1}^{N} x_{n, k, \ell} f_{n}\right\|_{B}^{q}\right)^{\frac{1}{q}} \\
& \leq M_{(q)}(B)\left\|\left(\sum_{\ell=1}^{L} \mathbf{P}\left(\Omega_{k, \ell}\right)\left|\sum_{n=1}^{N} x_{n, k, \ell} f_{n}\right|^{q}\right)^{\frac{1}{q}}\right\|_{B}
\end{aligned}
$$

Or on a évidemment pour tout $\left(t_{1}, \ldots, t_{N}\right) \in \mathbb{R}^{N}$

$$
\left(\sum_{\ell=1}^{L} \mathbf{P}\left(\Omega_{k, \ell}\right)\left|\sum_{n=1}^{N} x_{n, k, \ell} t_{n}\right|^{q}\right)^{\frac{1}{q}}=\mathbf{E}\left[\left|\sum_{n=1}^{N} X_{n, k} t_{n}\right|^{q}\right]^{\frac{1}{q}} .
$$


Si $k$ tend vers $+\infty$, l'expression précédente converge uniformément, en tant que fonction de $\left(t_{1}, \ldots, t_{N}\right)$, sur chaque compact de $\mathbb{R}^{N}$ vers

$$
\mathbf{E}\left[\left|\sum_{n=1}^{N} X_{n} t_{n}\right|^{q}\right]^{\frac{1}{q}}
$$

Le calcul fonctionnel du théorème 2.18 assure que l'égalité $(54)$ est encore valide en substituant $\left(f_{1}, \ldots, f_{N}\right)$ à $\left(t_{1}, \ldots, t_{N}\right)$, ce qui nous donne

$$
\mathbf{E}\left[\left\|\sum_{n=1}^{N} X_{n, k} f_{n}\right\|_{B}^{q}\right]^{\frac{1}{q}} \leq M_{(q)}(B)\left\|\mathbf{E}\left[\left|\sum_{n=1}^{N} X_{n, k} f_{n}\right|^{q}\right]^{\frac{1}{q}}\right\|_{B} .
$$

L'estimation (49) de continuité du calcul fonctionnel assure aussi que le membre droit de (55) tend vers

$$
M_{(q)}(B)\left\|\mathbf{E}\left[\left|\sum_{n=1}^{N} X_{n} f_{n}\right|^{q}\right]^{\frac{1}{q}}\right\|_{B}
$$

Enfin, il est clair que le membre gauche de (55) tend vers le membre gauche de (53). Pour démontrer (52), on refait la même démarche de densité dans $L^{1}(\Omega)$ à l'aide de l'inégalité triangulaire dans $B$ :

$$
\begin{aligned}
\mathbf{E}_{\omega}\left[\left\|\sum_{n=1}^{N} X_{n, k}(\omega) f_{n}\right\|_{B}\right] & =\sum_{\ell=1}^{L} \mathbf{P}\left(\Omega_{k, \ell}\right)\left\|\sum_{n=1}^{N} x_{n, k, \ell} f_{n}\right\|_{B}, \\
& \geq\left\|\sum_{\ell=1}^{L} \mathbf{P}\left(\Omega_{k, \ell}\right) \mid \sum_{n=1}^{N} x_{n, k, \ell} f_{n}\right\| \|_{B} .
\end{aligned}
$$

Corollaire 2.23. - Considérons des matrices aléatoires $M_{0}: \Omega \rightarrow \mathcal{M}_{d_{0}}(\mathbb{R}), \ldots, M_{N}: \Omega \rightarrow \mathcal{M}_{d_{N}}(\mathbb{R})$ dont les coefficients appartiennent à $L^{1}(\Omega)$ et des matrices $b_{0} \in \mathcal{M}_{d_{0}}(B), \ldots, b_{N} \in \mathcal{M}_{d_{N}}(B)$ dont les coefficients appartiennent à un treillis de Banach $B$. Alors on a l'inégalité :

$$
\left\|\mathbf{E}\left[\left|\sum_{n=0}^{N} \sqrt{d_{n}} \operatorname{tr}\left(M_{n} b_{n}\right)\right|\right]\right\|_{B} \leq \mathbf{E}\left[\left\|\sum_{n=0}^{N} \sqrt{d_{n}} \operatorname{tr}\left(M_{n} b_{n}\right)\right\|_{B}\right] .
$$

S'il existe de plus un réel $q \in[1,+\infty[$ tel que $B$ soit $q$-concave et tel que les coefficients des matrices aléatoires $M_{0}, \ldots, M_{N}$ appartiennent à $L^{q}(\Omega)$, alors on a

$$
\mathbf{E}\left[\left\|\sum_{n=0}^{N} \sqrt{d_{n}} \operatorname{tr}\left(M_{n} b_{n}\right)\right\|_{B}^{q}\right]^{\frac{1}{q}} \leq M_{(q)}(B)\left\|\mathbf{E}\left[\left|\sum_{n=0}^{N} \sqrt{d_{n}} \operatorname{tr}\left(M_{n} b_{n}\right)\right|^{q}\right]^{\frac{1}{q}}\right\|_{B} .
$$

Il nous reste à remarquer que les inégalités de Kahane-Khintchine-Marcus-Pisier permettent d'étendre le théorème de Maurey [37, Theorem 1.d.6, i)] au cas multidimensionnel.

Théorème 2.24. - Soit $B$ un treillis de Banach q-concave, avec $q \in[2,+\infty[$ et considérons une suite de matrices $b_{n} \in \mathcal{M}_{d_{n}}(B)$. Alors, pour tout entier $N \in \mathbb{N}$, on a

$$
\frac{1}{K_{2,1}}\left\|\sqrt{\sum_{n=0}^{N}\left\|b_{n}\right\|_{H S}^{2}}\right\|_{B} \leq \mathbf{E}\left[\left\|\sum_{n=0}^{N} \sqrt{d_{n}} \operatorname{tr}\left(\mathcal{E}_{n} b_{n}\right)\right\|_{B}\right] \leq M_{(q)}(B) K_{q, 2} \| \sqrt{\sum_{n=0}^{N}\left\|b_{n}\right\|_{H S}^{2} \|_{B},}
$$

où l'élément $\sqrt{\sum_{n=0}^{N}\left\|b_{n}\right\|_{H S}^{2}} \in B$ est défini par calcul fonctionnel. 
Preuve. La minoration est vraie sans hypothèse de $q$-concavité. La version scalaire des inégalités de KahaneKhintchine-Marcus-Pisier (voir (40) et (41)) rend triviale l'inégalité suivante si les matrices $b_{n}$ sont à coefficients réels

$$
\sqrt{\sum_{n=0}^{N}\left\|b_{n}\right\|_{H S}^{2}} \leq K_{2,1} \mathbf{E}\left[\left|\sum_{n=0}^{N} \sqrt{d_{n}} \operatorname{tr}\left(\mathcal{E}_{n} b_{n}\right)\right|\right] .
$$

L'inégalité précédente s'étend par calcul fonctionnel si les coefficients des matrices $b_{n}$ sont à coefficients dans $B$. La minoration de (56) découle alors du corollaire 2.23. On raisonne de même pour la majoration.

Le corollaire 2.23 et le théorème 2.24 nous permettent de prouver le théorème 2.21 . Il s'agit de reprendre mutatis mutandis les arguments de la partie 2.3 à partir du lemme 2.12 .

2.5. Preuves des théorèmes 2.5 et $\mathbf{2 . 6}$, partie I : rétracte d'un espace de Banach. - On conviendra que les éléments de $E_{n} \subset L^{2}(X)$ sont des fonctions de la variable $y \in X$ et l'on préférera les deux écritures

$$
L^{2}(X) \rightarrow L_{y}^{2}(X) \quad \text { et } \quad \mathbf{P L}^{p}\left(X, \oplus E_{n}\right) \rightarrow \mathbf{P} \mathbf{L}_{y}^{p}\left(X, \oplus E_{n}\right) .
$$

Il sera aussi commode de définir l'espace de Hilbert abstrait $\oplus E_{n}$, c'est-à-dire que l'on pose

$$
\forall\left(u_{n}\right)_{n \in \mathbb{N}} \in \bigoplus_{n \in \mathbb{N}} E_{n} \quad\left\|\left(u_{n}\right)_{n \in \mathbb{N}}\right\|_{\oplus E_{n}}:=\left(\int_{X} \sum_{n \in \mathbb{N}}\left|u_{n}(y)\right|^{2} d \mu(y)\right)^{\frac{1}{2}} .
$$

Concernant l'espace de Bochner-Lebesgue, on conservera la lettre $x \in X$ pour écrire $L_{x}^{p}\left(X, \oplus E_{n}\right)$ au lieu de $L^{p}\left(X, \oplus E_{n}\right)$. La définition 2.3 dit exactement que l'opérateur linéaire suivant est isométrique

$$
\begin{aligned}
S_{p}: \mathbf{P L}_{y}^{p}\left(X, \oplus E_{n}\right) & \rightarrow L_{x}^{p}\left(X, \oplus E_{n}\right) \\
\left(u_{n}(y)\right)_{n \in \mathbb{N}} & \mapsto\left(\frac{\sqrt{e_{n}(x)}}{\sqrt{d_{n}}} u_{n}(y)\right)_{n \in \mathbb{N}} .
\end{aligned}
$$

Le résultat suivant est facile.

Proposition 2.25. - L'espace $\mathbf{P} \mathbf{L}_{y}^{p}\left(X, \oplus E_{n}\right)$ est complet.

Preuve. Il s'agit de prouver que l'image de l'opérateur (57) est un sous-espace fermé de $L_{x}^{p}\left(X, \oplus E_{n}\right)$. Remarquons d'abord, pour tout $k \in \mathbb{N}$, la continuité du projecteur

$$
\begin{aligned}
L_{x}^{p}\left(X, \oplus E_{n}\right) & \rightarrow L_{x}^{p}\left(X, L_{y}^{2}(X)\right) \\
\left(w_{n}(x, y)\right)_{n \in \mathbb{N}} & \mapsto w_{k}(x, y) .
\end{aligned}
$$

Il suffit donc de prouver que $\left\{\sqrt{e_{n}(x)} u_{n}(y), \quad u_{n} \in E_{n}\right\}$ est un sous-espace vectoriel fermé de $L_{x}^{p}\left(X, L_{y}^{2}(X)\right)$ pour tout $n \in \mathbb{N}$. L'hypothèse $E_{n} \subset L^{p}(X)$ de la définition 2.3 signifie précisément que $\sqrt{e_{n}(x)}$ appartient à $L_{x}^{p}(X)$. Soit $\left(\sqrt{e_{n}(x)} u_{n, \ell}(y)\right)_{\ell \in \mathbb{N}}$ une suite convergente dans $L_{x}^{p}\left(X, L_{y}^{2}(X)\right)$ (avec $u_{n, \ell} \in E_{n}$ pour tout $\ell \in \mathbb{N})$. En particulier, $\left(u_{n, \ell}(y)\right)_{\ell \in \mathbb{N}}$ est de Cauchy dans $E_{n} \subset L_{y}^{2}(X)$ :

$$
\left\|u_{n, \ell}(y)-u_{n, \ell^{\prime}}(y)\right\|_{L_{y}^{2}(X)}=\frac{1}{\left\|\sqrt{e_{n}(x)}\right\|_{L_{x}^{p}}}\left\|\sqrt{e_{n}(x)}\left(u_{n, \ell}(y)-u_{n, \ell^{\prime}}(y)\right)\right\|_{L_{x}^{p}\left(X, L_{y}^{2}(X)\right)} .
$$

On déduit que $\left(u_{n, \ell}\right)_{\ell \in \mathbb{N}}$ converge dans $L^{2}(X)$ vers une fonction $u_{n, \infty} \in L^{2}(X)$ et bien entendu que l'on a $u_{n, \infty} \in E_{n}$ (car $E_{n}$ est de dimension finie). Il est alors immédiat que $\left(\sqrt{e_{n}(x)} u_{n, \ell}(y)\right)_{\ell \in \mathbb{N}}$ converge vers $\sqrt{e_{n}(x)} u_{n, \infty}(y)$ dans $L_{x}^{p}\left(X, L_{y}^{2}(X)\right)$.

Commençons par les points faciles ayant trait à la dualité et l'interpolation des espaces $\mathbf{P} \mathbf{L}^{p}\left(X, \oplus E_{n}\right)$.

\section{Dualité}


Rappelons que l'on note $q=\frac{p}{p-1}$ l'exposant conjugué de $p$. On montre facilement l'inégalité (38) pour tout $(u, w) \in \mathbf{P L}_{y}^{p}\left(X, \oplus E_{n}\right) \times \mathbf{P} \mathbf{L}_{y}^{q}\left(X, \oplus E_{n}\right):$

$$
\begin{aligned}
\sum_{n \geq 0}\left|\left\langle u_{n}, w_{n}\right\rangle_{L^{2}(X)}\right| & =\int_{X} \sum_{n \in \mathbb{N}} \frac{e_{n}(x)}{d_{n}}\left|\left\langle u_{n}, w_{n}\right\rangle_{L^{2}(X)}\right| d \mu(x) \\
& \leq \int_{X} \sqrt{\sum_{n \geq 0} \frac{e_{n}(x)}{d_{n}}\left\|u_{n}(y)\right\|_{L_{y}^{2}(X)}^{2}} \sqrt{\sum_{n \geq 0} \frac{e_{n}(x)}{d_{n}}\left\|w_{n}(y)\right\|_{L_{y}^{2}(X)}^{2}} d \mu(x) \\
& \leq\|u\|_{\mathbf{P L}^{p}\left(X, \oplus E_{n}\right)}\|w\|_{\mathbf{P L}^{q}\left(X, \oplus E_{n}\right)} \cdot
\end{aligned}
$$

L'injection canonique $\Lambda_{p}: \mathbf{P L}_{y}^{q}\left(X, \oplus E_{n}\right) \rightarrow \mathbf{P L}_{y}^{p}\left(X, \oplus E_{n}\right)^{\prime}$, définie dans l'énoncé du théorème 2.6, est donc continue.

\section{Interpolation}

Rappelons que les espaces $\mathbf{P} \mathbf{L}_{y}^{p}\left(X \oplus E_{n}\right)$ et leurs interpolés complexes et réels peuvent être vus comme des sous-espaces de $\prod_{n \in \mathbb{N}} E_{n}$. En outre, on a le résultat suivant [57, Part 1.18.4].

Théorème 2.26. - Considérons deux espaces de Banach complexes $B_{1}$ et $B_{2}$ ainsi que des réels $p_{1}<p<$ $p_{2}$ appartenant à $\left[1,+\infty[\right.$. En notant $\theta \in] 0,1\left[\right.$ le réel qui vérifie $\frac{1}{p}=\frac{1-\theta}{p_{1}}+\frac{\theta}{p_{2}}$, on a

$$
\left[L^{p_{1}}\left(X, B_{1}\right), L^{p_{2}}\left(X, B_{2}\right)\right]_{\theta}=L^{p}\left(X,\left[B_{1}, B_{2}\right]_{\theta}\right) .
$$

Si un opérateur linéaire $T$ borné de $L^{p_{1}}\left(X, B_{1}\right)$ dans lui-même et de $L^{p_{2}}\left(X, B_{2}\right)$ dans lui-même alors il est aussi borné de $L^{p}\left(X,\left[B_{1}, B_{2}\right]_{\theta}\right)$ dans lui-même. Le même énoncé est valide en remplaçant la méthode d'interpolation complexe $[\cdot, \cdot]_{\theta}$ par la méthode d'interpolation réelle $[\cdot, \cdot]_{\theta, p}$.

Avec les notations du théorème précédent, on peut interpoler l'application (57) et assurer que l'opérateur

$$
\begin{aligned}
{\left[\mathbf{P L}_{y}^{p_{1}}\left(X \oplus E_{n}\right), \mathbf{P L}_{y}^{p_{2}}\left(X, \oplus E_{n}\right)\right]_{\theta} } & \rightarrow L_{x}^{p}\left(X, \oplus E_{n}\right) \\
\left(u_{n}(y)\right)_{n \in \mathbb{N}} & \mapsto\left(\frac{\sqrt{e_{n}(x)}}{\sqrt{d_{n}}} u_{n}(y)\right)_{n \in \mathbb{N}}
\end{aligned}
$$

est borné. Cela implique l'inclusion continue

$$
\left[\mathbf{P L}_{y}^{p_{1}}\left(X \oplus E_{n}\right), \mathbf{P L}_{y}^{p_{2}}\left(X, \oplus E_{n}\right)\right]_{\theta} \subset \mathbf{P} \mathbf{L}_{y}^{p}\left(X, \oplus E_{n}\right) .
$$

\section{Vers la notion de rétracte d'un espace de Banach}

Cependant, il ne paraît pas évident d'aller plus loin dans les deux analyses précédentes, c'est-à-dire de prouver que $\Lambda_{p}: \mathbf{P L}_{y}^{q}\left(X, \oplus E_{n}\right) \rightarrow \mathbf{P L}_{y}^{p}\left(X, \oplus E_{n}\right)^{\prime}$ est surjective et que l'inclusion (58) est une égalité. La notion de rétracte d'un espace de Banach permet de reformuler la question.

Définition 2.27. - Soient $A$ et $B$ deux $\mathbb{C}$-espaces vectoriels normés, on dit que $A$ est un rétracte de $B$ s'il existe deux applications linéaires bornées $S: A \rightarrow B$ et $R: B \rightarrow A$ telles que $R S=i d_{A}$.

Dans la définition précédente, il faut imaginer que $B$ est un espace de référence qui est bien compris et $A$ un sous-espace que l'on veut analyser. Le prototype de rétracte qu'il faut avoir à l'esprit est le cas où $A$ est un sous-espace complémenté de $B$, c'est-à-dire image d'un projecteur borné, l'application $S$ est alors l'application identité et $R$ un projecteur de $B \operatorname{sur} A$. En effet, on vérifie facilement le résultat suivant.

Proposition 2.28. - Avec les mêmes notations que dans la définition 2.27, on a

i) $S R: B \rightarrow B$ est un projecteur borné, son espace image est égal à $S(A)$ et est fermé. 
ii) $S$ est un isomorphisme d'espaces vectoriels normés de $A$ sur $S(A) \subset B$ :

$$
\forall a \in A \quad \frac{1}{\|R\|_{B \rightarrow A}}\|a\|_{A} \leq\|S(a)\|_{B} \leq\|S\|_{A \rightarrow B}\|a\|_{A} .
$$

iii) si $B$ est complet alors $A$ l'est aussi.

En ce qui concerne la dualité, on a le corollaire facile.

Corollaire 2.29. - Avec les mêmes notations que dans la définition 2.27, si l'on note les applications duales (ou applications transposées) de $S$ et $R$ :

$$
\begin{aligned}
{ }^{t} S: B^{\prime} & \rightarrow A^{\prime}, & { }^{t} R: A^{\prime} & \rightarrow B^{\prime}, \\
\phi & \mapsto \phi \circ S & \psi & \mapsto \psi \circ R
\end{aligned}
$$

Alors on a ${ }^{t} S^{t} R=i d_{A^{\prime}}$. Cela signifie que l'espace dual $A^{\prime}$ est un rétracte de $B^{\prime}$ et en particulier que ${ }^{t} R$ est un isomorphisme de $A^{\prime}$ sur l'image du projecteur ${ }^{t} R^{t} S={ }^{t}(S R): B^{\prime} \rightarrow B^{\prime}$.

En ce qui concerne l'interpolation réelle ou complexe, la réponse est donnée par le résultat suivant [57, Page 22, Theorem 1.2.4].

Corollaire 2.30. - On note $[\cdot, \cdot \cdot]$ une méthode d'interpolation. Soient $\left(A_{1}, A_{2}\right)$ et $\left(B_{1}, B_{2}\right)$ deux couples d'interpolation d'espaces de Banach, on suppose qu'il existe un opérateur linéaire borné $S: A_{1} \rightarrow B_{1}$ et $S: A_{2} \rightarrow B_{2}$ et un opérateur linéaire borné $R: B_{1} \rightarrow A_{1}$ et $R: B_{2} \rightarrow A_{2}$ qui satisfont:

$$
\forall a \in A_{1}+A_{2} \quad R S(a)=a .
$$

Alors $S R$ est un projecteur borné de l'espace interpolé $\left[B_{1}, B_{2}\right]$ et $S$ réalise un isomorphisme d'espaces de Banach de l'espace interpolé $\left[A_{1}, A_{2}\right]$ sur $S R\left(\left[B_{1}, B_{2}\right]\right)$.

Preuve. C'est immédiat puisque l'espace de Banach $\left[A_{1}, A_{2}\right]$ est un rétracte de $\left[B_{1}, B_{2}\right]$ par l'intermédiaire des opérateurs bornés $S:\left[A_{1}, A_{2}\right] \rightarrow\left[B_{1}, B_{2}\right]$ et $R:\left[B_{1}, B_{2}\right] \rightarrow\left[A_{1}, A_{2}\right]$.

Pour comprendre les espaces duaux et interpolés des espaces $\mathbf{P} \mathbf{L}_{y}^{p}\left(X, \oplus E_{n}\right)$, il suffit d'étudier si $\mathbf{P} \mathbf{L}_{y}^{p}\left(X, \oplus E_{n}\right)$ est un rétracte de l'espace de Banach $L_{x}^{p}\left(X, \oplus E_{n}\right)$ par le biais de l'application $S_{p}$ définie en (57) si $p$ parcourt ]$p_{1}, p_{2}$ [ pour les théorèmes 2.5 et 2.6. En d'autres termes, on cherche un opérateur borné

$$
R_{p}: L_{x}^{p}\left(X, \oplus E_{n}\right) \rightarrow \mathbf{P L}_{y}^{p}\left(X, \oplus E_{n}\right)
$$

tel que $R_{p} S_{p}=\operatorname{id}_{\mathbf{P L}}^{p}\left(X, \oplus E_{n}\right)$.

\section{Stratégie pour le théorème 2.6 de dualité}

Puisque les fonctions $\frac{1}{d_{n}} e_{n}$ sont des densités de probabilité sur $X$, nous avons un candidat très naturel pour l'opérateur $R_{p}$. En effet, posons :

$$
\begin{aligned}
R_{p}: L_{x}^{p}\left(X, \oplus E_{n}\right) & \rightarrow \mathbf{P L}_{y}^{p}\left(X, \oplus E_{n}\right) \\
\left(u_{n}(x, y)\right)_{n \geq 0} & \mapsto\left(\int_{X} u_{n}\left(x^{\prime}, y\right) \frac{\sqrt{e_{n}\left(x^{\prime}\right)}}{\sqrt{d_{n}}} d \mu\left(x^{\prime}\right)\right)_{n \geq 0} .
\end{aligned}
$$

D'après la définition (57), on a bien formellement $R_{p} S_{p}=\operatorname{id}_{\mathbf{P}} \mathbf{L}_{y}^{p}\left(X, \oplus E_{n}\right)$. Malheureusement, nous ne voyons aucune raison triviale assurant que $R_{p}$ arrive bien dans $\mathbf{P L}_{y}^{p}\left(X, \oplus E_{n}\right)$ ! Puisque $S_{p}$ est une isométrie, la bornitude de $R_{p}$ équivaut à la bornitude du projecteur

$$
\begin{aligned}
S_{p} R_{p}: L_{x}^{p}\left(X, \oplus E_{n}\right) & \rightarrow L_{x}^{p}\left(X, \oplus E_{n}\right) \\
\left(u_{n}(x, y)\right)_{n \in \mathbb{N}} & \mapsto\left(\frac{\sqrt{e_{n}(x)}}{\sqrt{d_{n}}} \int_{X} u_{n}\left(x^{\prime}, y\right) \frac{\sqrt{e_{n}\left(x^{\prime}\right)}}{\sqrt{d_{n}}} d \mu\left(x^{\prime}\right)\right)_{n \in \mathbb{N}} .
\end{aligned}
$$

Par souci pédagogique, permettons-nous de considérer comme exemple le cas unidimensionnel :

$$
d_{n}=1, \quad e_{n}(x)=\left|\phi_{n}(x)\right|^{2}, \quad E_{n}=\mathbb{C} \phi_{n}, \quad \int_{X}\left|\phi_{n}(x)\right|^{2} d \mu(x)=1 .
$$


Dans ce cas, un élément de $L_{x}^{p}\left(X, \oplus E_{n}\right)$ est de la forme $\left(u_{n}(x) \phi_{n}(y)\right)_{n \in \mathbb{N}}$ et il est donc évident que $L_{x}^{p}\left(X, \oplus E_{n}\right)$ s'identifie à $L_{x}^{p}\left(X, \ell^{2}(\mathbb{N})\right)$ par le biais de l'isométrie

$$
\begin{aligned}
L_{x}^{p}\left(X, \oplus E_{n}\right) & \rightarrow L_{x}^{p}\left(X, \ell^{2}(\mathbb{N})\right) \\
\left(u_{n}(x) \phi_{n}(y)\right)_{n \in \mathbb{N}} & \mapsto\left(u_{n}(x)\right)_{n \in \mathbb{N}} .
\end{aligned}
$$

Par conséquent, la bornitude du projecteur $S_{p} R_{p}$ équivaut à celle du projecteur

$$
\begin{aligned}
L_{x}^{p}\left(X, \ell^{2}(\mathbb{N})\right) & \rightarrow L_{x}^{p}\left(X, \ell^{2}(\mathbb{N})\right) \\
\left(u_{n}(x)\right)_{n \in \mathbb{N}} & \mapsto\left(\left|\phi_{n}(x)\right| \int_{X} u_{n}\left(x^{\prime}\right)\left|\phi_{n}\left(x^{\prime}\right)\right| d \mu\left(x^{\prime}\right)\right)_{n \in \mathbb{N}} .
\end{aligned}
$$

Revenons au cas général et rappelons que sous les hypothèses du théorème 2.6 de dualité, $p_{1}$ et $p_{2}$ sont supposés être deux exposants conjugués. La bornitude du projecteur $S_{p} R_{p}$ sera prouvée dans la partie 2.8 pour tout $p \in] p_{1}, p_{2}$ [ grâce à (35) et (37). La proposition suivante achèvera la preuve du théorème 2.6 de dualité et explique pourquoi la notion de rétracte est la bonne notion pour aborder la dualité des espaces $\mathbf{P L}^{p}\left(X, \oplus E_{n}\right)$.

Proposition 2.31. - Fixons $p \in] 1,+\infty\left[\right.$ et posons $q=\frac{p}{p-1}$ l'exposant conjugué. Pour tout $n \in \mathbb{N}$, on suppose que $\sqrt{e_{n}}$ appartient à $L^{p}(X) \cap L^{q}(X)$. Alors les propriétés suivantes sont équivalentes :

i) l'injection canonique $\Lambda_{p}: \mathbf{P L}_{y}^{q}\left(X, \oplus E_{n}\right) \rightarrow \mathbf{P L}_{y}^{p}\left(X, \oplus E_{n}\right)^{\prime}$ est surjective,

ii) l'injection canonique $\Lambda_{q}: \mathbf{P} \mathbf{L}_{y}^{p}\left(X, \oplus E_{n}\right) \rightarrow \mathbf{P L}_{y}^{q}\left(X, \oplus E_{n}\right)^{\prime}$ est surjective,

iii) l'injection canonique $\Lambda_{p}: \mathbf{P L}_{y}^{q}\left(X, \oplus E_{n}\right) \rightarrow \mathbf{P L}_{y}^{p}\left(X, \oplus E_{n}\right)^{\prime}$ est un isomorphisme d'espaces de Banach,

iv) l'injection canonique $\Lambda_{q}: \mathbf{P L}_{y}^{p}\left(X, \oplus E_{n}\right) \rightarrow \mathbf{P L}_{y}^{q}\left(X, \oplus E_{n}\right)^{\prime}$ est un isomorphisme d'espaces de Banach,

v) le projecteur $S_{p} R_{p}$ est borné sur $L_{x}^{p}\left(X, \oplus E_{n}\right)$,

vi) le projecteur $S_{q} R_{q}$ est borné sur $L_{x}^{q}\left(X, \oplus E_{n}\right)$.

Les assertions précédentes impliquent les assertions suivantes

$$
\begin{gathered}
\sup _{n \in \mathbb{N}}\left\|\frac{\sqrt{e_{n}}}{\sqrt{d_{n}}}\right\|_{L^{p}(X)}\left\|\frac{\sqrt{e_{n}}}{\sqrt{d_{n}}}\right\|_{L(q(X)}<+\infty . \\
\left\|S_{p} R_{p}\right\|=\left\|S_{q} R_{q}\right\|=\left\|R_{p}\right\|=\left\|R_{q}\right\|=\left\|\Lambda_{p}^{-1}\right\|=\left\|\Lambda_{q}^{-1}\right\| \in[1,+\infty[.
\end{gathered}
$$

Preuve. Preuve de (61). Pour tout $n \in \mathbb{N}$, on fixe $\phi \in E_{n}$ vérifiant $\|\phi\|_{L_{y}^{2}(X)}=1$ et l'on note

$$
u(x, y)=(0, \ldots, 0, \underbrace{{\sqrt{e_{n}(x)}}^{q-1} \phi(y)}_{n}, 0, \ldots) \in L_{x}^{p}\left(X, \oplus E_{n}\right) .
$$

On peut alors écrire

$$
\begin{aligned}
& \left\|\frac{\sqrt{e_{n}(x)}}{\sqrt{d_{n}}}\right\|_{L_{x}^{p}(X)} \times\left|\int_{X} \frac{\sqrt{e_{n}\left(x^{\prime}\right)}}{\sqrt{d_{n}}} d \mu\left(x^{\prime}\right)\right| \\
& \left\|\frac{\sqrt{e_{n}(x)}}{\sqrt{d_{n}}}\right\|_{L_{x}^{p}(X)} \times \frac{1}{\sqrt{d_{n}}}\left\|\sqrt{e_{n}(x)}\right\|_{L_{x}^{q}(X)}^{q} \leq S_{p} R_{p}\|\times\|{\sqrt{e_{n}(x)}}^{q-1} \|_{L_{x}^{p}(X)} \\
& \left\|\frac{\sqrt{e_{n}(x)}}{\sqrt{d_{n}}}\right\|_{L_{x}^{p}(X)}\left\|\frac{\sqrt{e_{n}(x)}}{\sqrt{d_{n}}}\right\|_{L_{x}^{q}(X)} \leq\left\|S_{p} R_{p}\right\| \times\left\|\sqrt{e_{n}(x)}\right\|_{L_{x}^{q}(X)}^{q-1}
\end{aligned}
$$

Équivalence des propriétés i), ii), iii), iv), v) et vi).

i) $\Leftrightarrow$ iii) et ii) $\Leftrightarrow$ iv). Cela découle du théorème de l'application ouverte pour des bijections linéaires continues entre espaces de Banach.

v) $\Leftrightarrow$ vi $)$. Puisque $\oplus E_{n}$ est un espace de Hilbert, les espaces de Bochner-Lebesgue $L_{x}^{p}\left(X, \oplus E_{n}\right)$ et $L_{x}^{q}\left(X, \oplus E_{n}\right)$ sont duaux l'un de l'autre et la dualité naturelle est donnée par

$$
\forall(u, w) \in L_{x}^{p}\left(X, \oplus E_{n}\right) \times L_{x}^{q}\left(X, \oplus E_{n}\right) \quad\langle u, w\rangle:=\int_{X}\left[\sum_{n \in \mathbb{N}} \int_{X} u_{n}(x, y) w_{n}(x, y) d \mu(y)\right] d \mu(x) .
$$


Or l'on a

$$
\begin{aligned}
\int_{X}\left[\sum_{n \in \mathbb{N}} \int_{X}\left|u_{n}(x, y) \| w_{n}(x, y)\right| d \mu(y)\right] d \mu(x) & \leq \int_{X}\left[\sum_{n \in \mathbb{N}}\left\|u_{n}(x, y)\right\|_{L_{y}^{2}(X)}\left\|w_{n}(x, y)\right\|_{L_{y}^{2}(X)}\right] d \mu(x) \\
& \leq\|u\|_{L_{x}^{p}\left(X, \oplus E_{n}\right)}\|w\|_{L_{x}^{q}\left(X, \oplus E_{n}\right)} \\
& <+\infty
\end{aligned}
$$

Le théorème de Fubini assure que la dualité naturelle s'écrit aussi

$$
\langle u, w\rangle=\sum_{n \in \mathbb{N}} \int_{X \times X} u_{n}(x, y) w_{n}(x, y) d \mu(x) d \mu(y) .
$$

La définition (60) nous permet alors d'écrire

$$
\left\langle S_{p} R_{p} u, w\right\rangle=\sum_{n \in \mathbb{N}} \frac{1}{d_{n}} \int_{X \times X}\left[\sqrt{e_{n}(x)} \int_{X} u_{n}\left(x^{\prime}, y\right) \sqrt{e_{n}\left(x^{\prime}\right)} d \mu\left(x^{\prime}\right)\right] w_{n}(x, y) d \mu(x) d \mu(y) .
$$

Remarquons maintenant, pour tout $n \in \mathbb{N}$, la majoration triviale de

$$
\iiint_{X^{3}} \sqrt{e_{n}(x)}\left|w_{n}(x, y)\right| \sqrt{e_{n}\left(x^{\prime}\right)}\left|u_{n}\left(x^{\prime}, y\right)\right| d \mu(x) d \mu\left(x^{\prime}\right) d \mu(y)
$$

par

$$
\begin{aligned}
& \left(\int_{X} \sqrt{e_{n}(x)}\left\|w_{n}(x, y)\right\|_{L_{y}^{2}(X)} d \mu(x)\right) \times\left(\int_{X} \sqrt{e_{n}\left(x^{\prime}\right)}\left\|u_{n}\left(x^{\prime}, y\right)\right\|_{L_{y}^{2}(X)} d \mu\left(x^{\prime}\right)\right) \\
& \quad \leq\left\|\sqrt{e_{n}(x)}\right\|_{L_{x}^{p}(X)}\left\|w_{n}(x, y)\right\|_{L_{x}^{q}\left(X, L_{y}^{2}(X)\right)} \times\left\|\sqrt{e_{n}\left(x^{\prime}\right)}\right\|_{L_{x^{\prime}}^{q}(X)}\left\|u_{n}\left(x^{\prime}, y\right)\right\|_{L_{x^{\prime}}^{p}\left(X, L_{y}^{2}(X)\right)} \\
& \quad \leq\left\|\sqrt{e_{n}(x)}\right\|_{L_{x}^{p}(X)}\|w\|_{L_{x}^{q}\left(X, \oplus E_{n}\right)}\left\|\sqrt{e_{n}\left(x^{\prime}\right)}\right\|_{L_{x^{\prime}}^{q}(X)}\|u\|_{L_{x}^{p}\left(X, \oplus E_{n}\right)} \\
& \quad<+\infty .
\end{aligned}
$$

Le théorème de Fubini nous permet donc d'intervertir $x$ et $x^{\prime}$ pour obtenir

$$
\begin{aligned}
\left\langle S_{p} R_{p} u, w\right\rangle & =\sum_{n \in \mathbb{N}} \frac{1}{d_{n}} \int_{X \times X}\left[\sqrt{e_{n}(x)} \int_{X} w_{n}\left(x^{\prime}, y\right) \sqrt{e_{n}\left(x^{\prime}\right)} d \mu\left(x^{\prime}\right)\right] u_{n}(x, y) d \mu(x) d \mu(y) \\
& =\left\langle u, S_{q} R_{q} w\right\rangle .
\end{aligned}
$$

Ainsi, $S_{p} R_{p}$ et $S_{q} R_{q}$ sont adjoints l'un de l'autre. La continuité de l'un implique la continuité de l'autre.

Pour la fin de la démonstration, on aura besoin de l'expression de ${ }^{t} S_{p}$ (qui découle de (57)) :

$$
\begin{aligned}
{ }^{t} S_{p}: L_{x}^{q}\left(X, \oplus E_{n}\right) & \rightarrow \mathbf{P L}_{y}^{p}\left(X, \oplus E_{n}\right)^{\prime} \\
\left(w_{n}(x, y)\right)_{n \in \mathbb{N}} & \mapsto\left(\left(u_{n}(y)\right)_{n \geq 0} \mapsto \int_{X \times X} \sum_{n \in \mathbb{N}} w_{n}(x, y) u_{n}(y) \frac{\sqrt{e_{n}(x)}}{\sqrt{d_{n}}} d \mu(x) d \mu(y)\right) .
\end{aligned}
$$

iii) $\Rightarrow$ vi). À l'aide de (59), on vérifie la formule $R_{q}=\Lambda_{p}^{-1} \circ{ }^{t} S_{p}$. A fortiori, $S_{q} R_{q}$ est un opérateur borné.

iv) $\Rightarrow \mathrm{v}$ ). On permute $p$ et $q$ dans l'argument précédent.

vi) $\Rightarrow$ i). L'équivalence v) $\Leftrightarrow$ vi) assure que $S_{p} R_{p}$ est borné. Puisque $S_{p}$ et $S_{q}$ sont des isométries, $R_{p}$ et $R_{q}$ sont des opérateurs bornés et l'on a par construction

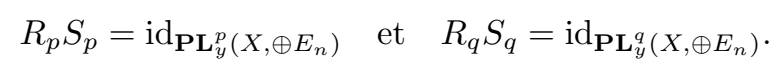

L'idée est d'exprimer $\Lambda_{p}$ avec la formule suivante qui découle facilement de (57) :

$$
\Lambda_{p}={ }^{t} S_{p} S_{q} .
$$

Utilisant que $S_{p} R_{p}$ et $S_{q} R_{q}$ sont deux opérateurs duaux, on obtient

$$
\begin{aligned}
{ }^{t} R_{p} \Lambda_{p} & ={ }^{t} R_{p}{ }^{t} S_{p} S_{q} \\
& ={ }^{t}\left(S_{p} R_{p}\right) S_{q} \\
& =S_{q} R_{q} S_{q} \\
& =S_{q} .
\end{aligned}
$$

Le diagramme suivant est donc commutatif : 


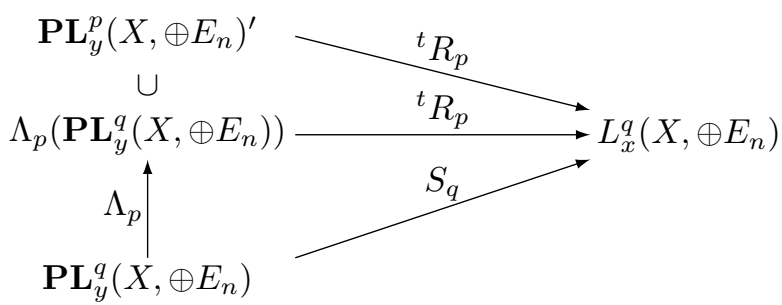

On doit examiner le diagramme précédent en se rappelant que l'on a

$$
{ }^{t} S_{p}{ }^{t} R_{p}=\operatorname{id}_{\mathbf{P L}}^{p}\left(X, \oplus E_{n}\right)^{\prime} \quad \text { et } \quad R_{q} S_{q}=\operatorname{id}_{\mathbf{P L}_{y}^{q}\left(X, \oplus E_{n}\right)} .
$$

La proposition 2.28 et le corollaire 2.29 assurent que $S_{q}$ réalise un isomorphisme d'espaces de Banach de $\mathbf{P} \mathbf{L}_{y}^{q}\left(X, \oplus E_{n}\right)$ sur l'image du projecteur $S_{q} R_{q}$ de $L_{x}^{q}\left(X, \oplus E_{n}\right)$ et que ${ }^{t} R_{p}$ réalise un isomorphisme d'espaces de Banach de $\mathbf{P L}_{y}^{p}\left(X, \oplus E_{n}\right)^{\prime}$ sur l'image du même projecteur ${ }^{t} R_{p}{ }^{t} S_{p}=S_{q} R_{q}$ de $L_{x}^{q}\left(X, \oplus E_{n}\right)$. Grâce à (63), on vérifie que $\Lambda_{p}\left(\mathbf{P} \mathbf{L}_{y}^{q}\left(X, \oplus E_{n}\right)\right)$ et $\mathbf{P} \mathbf{L}_{y}^{p}\left(X, \oplus E_{n}\right)^{\prime}$ ont la même image par l'opérateur ${ }^{t} R_{p}$ :

$$
\begin{aligned}
{ }^{t} R_{p}\left(\Lambda_{p}\left(\mathbf{P L}_{y}^{q}\left(X, \oplus E_{n}\right)\right)\right) & =S_{q}\left(\mathbf{P L}_{y}^{q}\left(X, \oplus E_{n}\right)\right)=S_{q} R_{q}\left(L_{x}^{q}\left(X, \oplus E_{n}\right)\right), \\
{ }^{t} R_{p}\left(\mathbf{P L}_{y}^{p}\left(X, \oplus E_{n}\right)^{\prime}\right) & =S_{q} R_{q}\left(L_{x}^{q}\left(X, \oplus E_{n}\right)\right) .
\end{aligned}
$$

Par application de ${ }^{t} S_{p}$, on obtient i) :

$$
\Lambda_{p}\left(\mathbf{P L}_{y}^{q}\left(X, \oplus E_{n}\right)\right)=\mathbf{P L}_{y}^{p}\left(X, \oplus E_{n}\right)^{\prime} .
$$

v) $\Rightarrow$ ii). On permute $p$ et $q$ dans la preuve précédente.

Preuve de (62). Comme $S_{p} R_{p}$ est un projecteur, sa norme est supérieure ou égale à 1 . On a déjà vu au cours de la démonstration précédente l'égalité $\left\|S_{p} R_{p}\right\|=\left\|S_{q} R_{q}\right\|$. Comme $S_{p}$ et $S_{q}$ sont des isométries, on déduit à la fois la formule $\left\|S_{p} R_{p}\right\|=\left\|R_{p}\right\|$ et $\left\|S_{q} R_{q}\right\|=\left\|R_{q}\right\|$ et la formule $\left\|R_{p}\right\|=\left\|^{t} R_{p}\right\|=\left\|\Lambda_{p}^{-1}\right\|$ (grâce à $(63))$.

\section{Stratégie pour le théorème 2.5}

Il est naturel d'espérer utiliser une stratégie similaire pour prouver le théorème 2.5 d'interpolation en utilisant cette fois-ci le corollaire 2.30. On suppose donc seulement que l'on a $\frac{1}{p_{1}}+\frac{1}{p_{2}} \leq 1$. La proposition 2.31 nous informe que la bornitude de $R_{p}$ pour tout $\left.p \in\right] p_{1}, p_{2}$ [ implique l'assertion (61) :

$$
\forall p \in] p_{1}, p_{2}\left[\quad \sup _{n \in \mathbb{N}}\left\|\frac{\sqrt{e_{n}}}{\sqrt{d_{n}}}\right\|_{L^{p}(X)}\left\|\frac{\sqrt{e_{n}}}{\sqrt{d_{n}}}\right\|_{L^{\frac{p}{p-1}}(X)}<+\infty .\right.
$$

En raison de la symétrie entre $p$ et $\frac{p}{p-1}$, les inégalités précédentes sont aussi valides si $p$ parcourt le plus petit intervalle contenant $] p_{1}, p_{2}$ [ et stable par la fonction $p \mapsto \frac{p}{p-1}$. Toutes ces inégalités sont trop violentes puisque l'on doit seulement se contenter de l'hypothèse (36). L'application $R_{p}$ parait donc inutilisable. Pour pallier ce problème, nous allons remplacer $R_{p}$ par une application de la forme

$$
\begin{aligned}
R_{p, \psi}: L_{x}^{p}\left(X, \oplus E_{n}\right) & \rightarrow \mathbf{P L}_{y}^{p}\left(X, \oplus E_{n}\right) \\
\left(u_{n}(x, y)\right)_{n \in \mathbb{N}} & \mapsto\left(\int_{X} u_{n}\left(x^{\prime}, y\right) \psi_{n}\left(x^{\prime}\right) d \mu\left(x^{\prime}\right)\right)_{n \geq 0},
\end{aligned}
$$

où $\left(\psi_{n}\right)_{n \in \mathbb{N}}$ est une suite de fonctions de $L^{\frac{p_{2}}{p_{2}-1}}(X) \cap L^{\frac{p_{1}}{p_{1}-1}}(X)$ vérifiant

$$
\int_{X} \frac{\sqrt{e_{n}(x)}}{\sqrt{d_{n}}} \psi_{n}(x) d \mu(x)=1
$$


De nouveau, on a bien $R_{p, \psi} S_{p}=\operatorname{id}_{\mathbf{P L}_{y}^{p}\left(X, \oplus E_{n}\right)}$ de manière formelle et l'on rencontre le même obstacle : il n'y a aucune raison pour que $R_{p, \psi}$ arrive bien dans $\mathbf{P L}_{y}^{p}\left(X, \oplus E_{n}\right)$. Une nouvelle fois, puisque $S_{p}$ est une isométrie pour tout $p \in] p_{1}, p_{2}$ [, la bornitude de $R_{p, \psi}$ équivaut à la bornitude de l'opérateur

$$
\begin{aligned}
S_{p} R_{p, \psi}: L_{x}^{p}\left(X, \oplus E_{n}\right) & \rightarrow L_{x}^{p}\left(X, \oplus E_{n}\right) \\
\left(u_{n}(x, y)\right)_{n \in \mathbb{N}} & \mapsto\left(\frac{\sqrt{e_{n}(x)}}{\sqrt{d_{n}}} \int_{X} u_{n}\left(x^{\prime}, y\right) \psi_{n}\left(x^{\prime}\right) d \mu\left(x^{\prime}\right)\right) .
\end{aligned}
$$

Sous les hypothèses (35) et (36), l'existence d'une suite adéquate $\left(\psi_{n}\right)_{n \in \mathbb{N}}$ et la bornitude de $S_{p} R_{p, \psi}$, pour $p \in] p_{1}, p_{2}[$, seront établies dans la partie 2.10 .

On peut maintenant expliquer la preuve du théorème 2.5 d'interpolation. On remarque que les expressions de $S_{p}$ et $R_{p, \psi}$ sont indépendantes de $p$. On fixe alors $p_{1}^{\prime}$ et $p_{2}^{\prime}$ deux réels appartenant à $] p_{1}, p_{2}[$. Pour tout $p \in] p_{1}^{\prime}, p_{2}^{\prime}\left[\right.$ on note $\theta^{\prime} \in[0,1]$ l'unique réel vérifiant $\frac{1}{p}=\frac{1-\theta^{\prime}}{p_{1}^{\prime}}+\frac{\theta^{\prime}}{p_{2}^{\prime}}$. Le corollaire 2.30 assure que l'image de l'opérateur

$$
S_{p}:\left[\mathbf{P L}_{y}^{p_{1}^{\prime}}\left(X, \oplus E_{n}\right), \mathbf{P L}_{y}^{p_{2}^{\prime}}\left(X, \oplus E_{n}\right)\right]_{\theta^{\prime}} \rightarrow\left[L_{x}^{p_{1}^{\prime}}\left(X, \oplus E_{n}\right), L_{x}^{p_{2}^{\prime}}\left(X, \oplus E_{n}\right)\right]_{\theta^{\prime}}
$$

est $S_{p} R_{p, \psi}\left(\left[L_{x}^{p_{1}^{\prime}}\left(X, \oplus E_{n}\right), L_{x}^{p_{2}^{\prime}}\left(X, \oplus E_{n}\right)\right]_{\theta^{\prime}}\right)$ et que $S_{p}$ induit un isomorphisme sur son image. Le théorème 2.26 et le point i) de la proposition 2.28 donnent alors

$$
\begin{aligned}
S_{p}\left(\left[\mathbf{P L}_{y}^{p_{1}^{\prime}}\left(X, \oplus E_{n}\right), \mathbf{P L}_{y}^{p_{2}^{\prime}}\left(X, \oplus E_{n}\right)\right]_{\theta^{\prime}}\right) & =S_{p} R_{p, \psi}\left(\left[L_{x}^{p_{1}^{\prime}}\left(X, \oplus E_{n}\right), L_{x}^{p_{2}^{\prime}}\left(X, \oplus E_{n}\right)\right]_{\theta^{\prime}}\right) \\
& =S_{p} R_{p, \psi}\left(L_{x}^{p}\left(X, \oplus E_{n}\right)\right) \\
& =S_{p}\left(\mathbf{P} \mathbf{L}_{y}^{p}\left(X, \oplus E_{n}\right)\right) .
\end{aligned}
$$

Se rappelant l'inclusion (58), on a donc le schéma

$$
\begin{gathered}
\mathbf{P L}_{y}^{p}\left(X, \oplus E_{n}\right) \stackrel{S_{p}}{\cup} \underset{S_{p}}{\longrightarrow} L_{x}^{p}\left(X, \oplus E_{n}\right) \\
{\left[\mathbf{P L}_{y}^{p_{1}^{\prime}}\left(X, \oplus E_{n}\right), \mathbf{P} \mathbf{L}_{y}^{p_{2}^{\prime}}\left(X, \oplus E_{n}\right)\right]_{\theta^{\prime}}}
\end{gathered}
$$

Or $S_{p}$ est isométrique sur $\mathbf{P} \mathbf{L}_{y}^{p}\left(X, \oplus E_{n}\right)$ et donc injectif. Cela nous amène à l'égalité

$$
\left[\mathbf{P L}_{y}^{p_{1}^{\prime}}\left(X, \oplus E_{n}\right), \mathbf{P} \mathbf{L}_{y}^{p_{2}^{\prime}}\left(X, \oplus E_{n}\right)\right]_{\theta^{\prime}}=\mathbf{P} \mathbf{L}_{y}^{p}\left(X, \oplus E_{n}\right)
$$

Enfin, les normes des espaces $\left[\mathbf{P} \mathbf{L}_{y}^{p_{1}^{\prime}}\left(X, \oplus E_{n}\right), \mathbf{P L}_{y}^{p_{2}^{\prime}}\left(X, \oplus E_{n}\right)\right]_{\theta^{\prime}}$ et $\mathbf{P} \mathbf{L}_{y}^{p}\left(X, \oplus E_{n}\right)$ sont équivalentes d'après le théorème du graphe fermé et l'inclusion continue (58). On aurait aussi pu invoquer le fait que $S_{p}$ est un isomorphisme d'espaces de Banach de $\left[\mathbf{P L}_{y}^{p_{1}^{\prime}}\left(X, \oplus E_{n}\right), \mathbf{P} \mathbf{L}_{y}^{p_{2}^{\prime}}\left(X, \oplus E_{n}\right)\right]_{\theta^{\prime}}$ sur son image. La même argumentation est valide en remplaçant la méthode d'interpolation complexe $[\cdot, \cdot]_{\theta^{\prime}}$ par la méthode d'interpolation réelle $[\cdot, \cdot]_{\theta^{\prime}, p}$.

2.6. Preuves des théorèmes 2.5 et $\mathbf{2 . 6}$, partie II : espaces de Lorentz. — On effectue quelques rappels sur les espaces de Lorentz $L^{p, \infty}(X)$, avec $\left.p \in\right] 1,+\infty[$ (voir par exemple [26, Chapter 1]). Afin d'exprimer l'inégalité de Hölder des espaces de Lorentz, il sera utile de remarquer la reformulation suivante de la quasi-norme $\|\cdot\|_{L^{p, \infty}(X)}$ :

$$
\begin{aligned}
& \forall f \in L^{p, \infty}(X) \quad\|f\|_{L^{p, \infty}(X)} \quad:=\inf \left\{c>0, \quad \forall t>0 \quad \mu\{x, \in X,|f(x)|>t\} \leq \frac{c^{p}}{t^{p}}\right\} \\
& =\sup _{T>0} T^{\frac{1}{p}} f^{\star}(T),
\end{aligned}
$$

où $f^{\star}:[0,+\infty[\rightarrow[0,+\infty[$ est le réarrangement décroissant de $f$ définie par la formule

$$
f^{\star}(T):=\inf \{t>0, \quad \mu\{|f|>t\} \leq T\} .
$$


En général, la quasi-norme $\|\cdot\|_{L^{p, \infty}(X)}$ ne vérifie pas l'inégalité triangulaire mais est toujours équivalente à la norme suivante dès lors que l'on a $p>1$ ([26, Page 13 and Page 64] ou [25, Part V, Lemma 2.8]) :

$$
\forall f \in L^{p, \infty}(X) \quad\|f\|_{L^{p, \infty}(X)}:=\sup _{\substack{A \in \mathcal{B}(X) \\ 0<\mu(A)<+\infty}} \frac{\left\|f \mathbf{1}_{A}\right\|_{L^{1}(X)}}{\mu(A)^{1-\frac{1}{p}}},
$$

où $\mathcal{B}(X)$ désigne l'ensemble des parties mesurables de $X$. Précisément, nous avons

$$
\|f\|_{L^{p, \infty}(X)} \leq\|f\|_{L^{p, \infty}(X)} \leq \frac{p}{p-1}\|f\|_{L^{p, \infty}(X)} .
$$

En d'autres termes, quitte à perdre une constante multiplicative, on pourra utiliser l'inégalité triangulaire dans $L^{p, \infty}(X)$. On aura aussi besoin de l'espace de Lorentz $L^{p, 1}(X)$ : il s'agit de l'espace vectoriel des fonctions mesurables $g: X \rightarrow \mathbb{C}$ qui vérifient

$$
\|g\|_{L^{p, 1}(X)}:=\int_{0}^{+\infty} T^{\frac{1}{p}} g^{\star}(T) \frac{d T}{T}<+\infty .
$$

Venons-en maintenant aux inégalités de Hardy-Littlewood et de Hölder, on a pour toutes fonctions $f \in$ $L^{p, \infty}(X)$ et $g \in L^{\frac{p}{p-1}, 1}(X)$

$$
\begin{aligned}
\left|\int_{X} f(x) g(x) d \mu(x)\right| & \leq \int_{0}^{+\infty} f^{\star}(T) g^{\star}(T) d T=\int_{0}^{+\infty} T^{\frac{1}{p}} f^{\star}(t) T^{\frac{p-1}{p}} g^{\star}(T) \frac{d T}{T} \\
& \leq\|f\|_{L^{p, \infty}(X)}\|g\|_{L^{\frac{p}{p-1}, 1}(X)} \\
& \leq\|f\|_{L^{p, \infty}(X)}\|g\|_{L^{\frac{p}{p-1}, 1}(X)} .
\end{aligned}
$$

On peut aussi définir des espaces de Lorentz $L^{p, r}(X, B)$, avec $r \in\{1, \infty\}$ (et même tout réel $r \geq 1$ ), à valeurs dans un espace de Banach complexe $B$. Il s'agit des fonctions mesurables $f: X \rightarrow B$ telles que la fonction $x \mapsto\|f(x)\|_{B}$ appartient à $L^{p, r}(X)$. La théorie de l'interpolation réelle fait jouer un rôle important aux espaces de Lorentz, en particulier on a le résultat suivant [57, Part 1.18.6, Lemma, Theorem 2, line (16), $q=p$ and Part 1.18.7, Theorem 2].

Théorème 2.32. - Considérons un espace de Banach complexe $B$ et trois réels $p_{1}<p<p_{2}$ appartenant $\grave{a}] 1,+\infty[$. Soit $\theta \in] 0,1\left[\right.$ l'unique réel tel que $\frac{1}{p}=\frac{1-\theta}{p_{1}}+\frac{\theta}{p_{2}}$. Pour tout $r \in\{1,+\infty\}$, on a pour la méthode d'interpolation réelle

$$
\left[L^{p_{1}, r}(X, B), L^{p_{2}, r}(X, B)\right]_{\theta, p}=L^{p}(X, B) .
$$

Si un opérateur linéaire $T$ est borné de $L^{p_{1}, 1}(X, B)$ à valeurs dans $L^{p_{1}, \infty}(X, B)$ et de $L^{p_{2}, 1}(X, B)$ à valeurs dans $L^{p_{2}, \infty}(X, B)$ alors il est borné de $L^{p}(X, B)$ à valeurs dans $L^{p}(X, B)$ et il existe une constante $K>0$ indépendante de $T$ telle que

$$
\|T\|_{L^{p}(X, B) \rightarrow L^{p}(X, B)} \leq K\|T\|_{L^{p_{1}, 1}(X, B) \rightarrow L^{p_{1}, \infty}(X, B)}^{1-\theta}\|T\|_{L^{p_{2}, 1}(X, B) \rightarrow L^{p_{2}, \infty}(X, B)}^{\theta} .
$$

2.7. Preuves des théorèmes 2.5 et 2.6 , partie III : $R$-bornitude. — Énonçons deux faits qui vont justifier que la bornitude d'un opérateur linéaire sur $L_{x}^{p}\left(X, \ell^{2}(\mathbb{N})\right)$ n'est généralement pas simple et qui vont motiver l'approche qui va suivre.

Fait 1 : soit $\left(P_{n}\right)_{n \geq 0}$ une suite d'opérateurs uniformément bornés sur $L^{2}(X)$, c'est-à-dire que l'on a

$$
\sup _{n \geq 0}\left\|P_{n}\right\|_{L^{2}(X) \rightarrow L^{2}(X)}<+\infty
$$

alors l'opérateur $\oplus P_{n}$ défini par l'expression suivante est borné sur $L^{2}\left(X, \ell^{2}(\mathbb{N})\right)$ :

$$
\forall\left(f_{n}\right)_{n \geq 0} \in L^{2}\left(X, \ell^{2}(\mathbb{N})\right) \quad\left(\oplus P_{n}\right)\left(f_{n}\right)=\left(P_{n} f_{n}\right) .
$$

En effet, cela découle immédiatement des formules :

$$
\left\|\left(f_{n}\right)\right\|_{L^{2}\left(X, \ell^{2}(\mathbb{N})\right)}=\sqrt{\int_{X} \sum_{n \geq 0}\left|f_{n}(x)\right|^{2} d \mu(x)}=\sqrt{\sum_{n \geq 0}\left\|f_{n}\right\|_{L^{2}(X)}^{2}} .
$$


Fait 2 : pour tout réel $p>2$ il existe une suite $\left(P_{n}\right)_{n \geq 0}$ d'opérateurs uniformément bornés de $L^{p}(\mathbb{R})$ et tels que l'opérateur $\oplus P_{n}$ défini par

$$
\forall\left(f_{n}\right)_{n \geq 0} \in L^{p}\left(X, \ell^{2}(\mathbb{N})\right) \quad\left(\oplus P_{n}\right)\left(f_{n}\right)=\left(P_{n} f_{n}\right) .
$$

n'est pas borné sur $L^{p}\left(\mathbb{R}, \ell^{2}(\mathbb{N})\right)$.

L'exemple est élémentaire. On considère les isométries $P_{n}$ définies par

$$
\forall n \in \mathbb{N} \quad \forall f \in L^{p}(\mathbb{R}) \quad \forall x \in \mathbb{R} \quad\left(P_{n} f\right)(x)=f(x+n),
$$

puis les fonctions $f_{N, n}$, paramétrées par $(n, N) \in \mathbb{N}^{2}$, définies par

$$
\forall x \in \mathbb{R} \quad f_{N, n}(x):=\left\{\begin{array}{rll}
\mathbf{1}_{[n, n+1[}(x) & \text { si } & n<N \\
0 & \text { si } & n \geq N
\end{array}\right.
$$

On a immédiatement $\left\|P_{n}\right\|_{L^{p}(X) \rightarrow L^{p}(X)}=1$. De même pour tout $N \in \mathbb{N}^{\star}$, on a

$$
\begin{aligned}
\left\|\left(\oplus P_{n}\right)\left(f_{N, n}\right)\right\|_{L^{p}\left(\mathbb{R}, \ell^{2}(\mathbb{N})\right)}^{p} & =\int_{\mathbb{R}}\left(\sum_{n=0}^{N-1} \mathbf{1}_{[0,1[}(x)\right)^{\frac{p}{2}} d x=N^{\frac{p}{2}}, \\
\left\|\left(f_{N, n}\right)\right\|_{L^{p}\left(\mathbb{R}, \ell^{2}(\mathbb{N})\right.}^{p} & =\int_{\mathbb{R}}\left(\sum_{n=0}^{N-1} \mathbf{1}_{[n, n+1[}(x)\right)^{\frac{p}{2}} d x=N \ll N^{\frac{p}{2}} .
\end{aligned}
$$

En faisant tendre $N$ vers $+\infty$, on voit que l'opérateur $\oplus P_{n}$ n'est pas borné sur $L^{p}\left(\mathbb{R}, \ell^{2}(\mathbb{N})\right)$. L'exemple précédent utilise le fait que $\mathbb{R}$ n'est pas compact afin de s'échapper vers l'infini. En réalité, cela est un faux-semblant et l'on peut transférer la construction précédente sur $L^{p}(0,1)$ à l'aide d'une isométrie linéaire surjective adéquate de $L^{p}(0,1)$ sur $L^{p}(\mathbb{R})$. En outre, si l'on raisonne par dualité ou si l'on choisit $f_{n}=\mathbf{1}_{[0,1[}$ à la place de $\mathbf{1}_{[n, n+1}$, la construction précédente s'adapte au cas $p<2$. Ainsi, le Fait $\mathbf{2}$ contraste fortement avec le Fait 1 et montre que la seule condition

$$
\sup _{n \in \mathbb{N}}\left\|P_{n}\right\|_{L^{p}(X) \rightarrow L^{p}(X)}<+\infty
$$

ne suffit pas pour assurer la bornitude de l'opérateur $\oplus P_{n}$ sur $L^{p}\left(X, \ell^{2}(\mathbb{N})\right)$ pour $p \neq 2$, même si $\mu(X)<$ $+\infty$. Un exemple qui illustre ce problème est la non continuité du multiplicateur de Fourier sur $L^{p}\left(\mathbb{R}^{2}\right)$ de l'indicatrice de la boule unité (voir la preuve de [21] dans laquelle les opérateurs $P_{n}$ sont des projecteurs de $\left.L^{p}\left(\mathbb{R}^{2}\right)\right)$.

La notion mathématique qui s'est dégagée est la $\mathcal{R}$-bornitude. Étant donné un espace de Banach $B$, une suite $\left(P_{n}\right)_{n \geq 0}$ d'opérateurs linéaires bornés de $B$ est dite $\mathcal{R}$-bornée s'il existe $K>0$ telle que pour toute suite $\left(f_{n}\right)_{n \in \mathbb{N}}$ de $B$ (nulle pour $n \gg 1$ ) on a un principe de contraction :

$$
\mathbf{E}\left[\left\|\sum_{n \geq 0} \varepsilon_{n} P_{n}\left(f_{n}\right)\right\|_{B}\right] \leq K \mathbf{E}\left[\left\|\sum_{n \geq 0} \varepsilon_{n} f_{n}\right\|_{B}\right] .
$$

Dans le cas $B=L^{p}(X)$, il est classique que les deux espérances précédentes s'estiment avec le théorème de Fubini et les inégalités de Kahane-Khintchine dans $L^{p}(X)$ et dans $\mathbb{C}$ (voir (43) dans le cas unidimensionnel). L'inégalité précédente signifie alors que l'opérateur $\oplus P_{n}$, défini en $(67)$, est borné sur $L^{p}\left(X, \ell^{2}(\mathbb{N})\right)$. Quitte à changer $K>0$, cela équivaut à une estimation de la forme

$$
\int_{X}\left(\sum_{n \geq 0}\left|\left(P_{n} f_{n}\right)(x)\right|^{2}\right)^{\frac{p}{2}} d \mu(x) \leq K \int_{X}\left(\sum_{n \geq 0}\left|f_{n}(x)\right|^{2}\right)^{\frac{p}{2}} d \mu(x) .
$$

Différents critères de $\mathcal{R}$-bornitude existent dans la littérature (voir par exemple [25, Chapter V], [60, Section 2], [15] et les références indiquées). Le lemme 2.33 donne un nouveau critère qui s'apparente au lemme de Schur et qui donne des conditions suffisantes pour obtenir la $\mathcal{R}$-bornitude. Ce critère suffira pour la théorie de l'interpolation et de la dualité des espaces $\mathbf{P L}^{p}\left(X, \oplus E_{n}\right)$ dans le cas unidimensionnel $d_{n}=\operatorname{dim}\left(E_{n}\right)=1$. Pour comprendre le cas $d_{n} \neq 1$, nous aurons besoin d'une version légèrement plus sophistiquée du lemme 2.33, à savoir le lemme 2.34, mais le coeur de l'idée est dans la démonstration suivante. 
Lemme 2.33. - Fixons des réels $p_{1}<p<p_{2}$ appartenant à $] 1,+\infty\left[\right.$. Notons $q_{1}:=\frac{p_{1}}{p_{1}-1}$ et $q_{2}:=\frac{p_{2}}{p_{2}-1}$. Considérons $K_{n}: X^{2} \rightarrow \mathbb{C}$ des fonctions mesurables, pour $n \in \mathbb{N}$, de sorte que l'on a

$$
\begin{aligned}
& \sup _{n \geq 0}\left\|K_{n}\left(x, x^{\prime}\right)\right\|_{L_{x^{\prime}}^{q_{1}, \infty}(X)} \in L_{x}^{p_{1}, \infty}(X), \\
& \sup _{n \geq 0}\left\|K_{n}\left(x, x^{\prime}\right)\right\|_{L_{x^{\prime}}^{q_{2}, \infty}(X)} \in L_{x}^{p_{2}, \infty}(X), \\
& \sup _{n \geq 0}\left\|K_{n}\left(x, x^{\prime}\right)\right\|_{L_{x}^{p_{1}, \infty}(X)} \in L_{x^{\prime}}^{q_{1}, \infty}(X), \\
& \sup _{n \geq 0}\left\|K_{n}\left(x, x^{\prime}\right)\right\|_{L_{x}^{p_{2}, \infty}(X)} \in L_{x^{\prime}}^{q_{2}, \infty}(X) .
\end{aligned}
$$

Les deux assertions suivantes sont vraies

a) pour tout entier $n \in \mathbb{N}$ l'opérateur $P_{n}$ de noyau $K_{n}$ défini par

$$
\forall f \in L_{x}^{p}(X) \quad \forall x \in X \quad\left(P_{n} f\right)(x)=\int_{X} K_{n}\left(x, x^{\prime}\right) f\left(x^{\prime}\right) d \mu\left(x^{\prime}\right),
$$

est borné sur $L^{p}(X)$ et la borne supérieure $\sup _{n \geq 0}\left\|P_{n}\right\|_{L^{p}(X) \rightarrow L^{p}(X)}$ est finie.

b) la famille d'opérateurs $\left(P_{n}\right)_{n \in \mathbb{N}}$ de $L^{p}(X)$ est $\mathcal{R}$-bornée.

PREuve. Nous aurons besoin des propriétés des espaces de Lorentz rappelées dans la partie 2.6.

a) On affirme que (68) implique la bornitude de l'opérateur

$$
P_{n}: L_{x}^{p_{1}, 1}(X) \rightarrow L_{x}^{p_{1}, \infty}(X) .
$$

En effet, l'inégalité de Hölder (66) donne

$$
\begin{aligned}
\left|\left(P_{n} f\right)(x)\right| & \leq\left\|K_{n}\left(x, x^{\prime}\right)\right\|_{L_{x^{\prime}}^{q_{1}, \infty}(X)}\|f\|_{L^{p_{1}, 1}(X)} \\
\left\|P_{n} f\right\|_{L^{p_{1}, \infty}(X)} & \leq\|\| K_{n}\left(x, x^{\prime}\right)\left\|_{L_{x^{\prime}}^{q_{1}, \infty}(X)}\right\|_{L_{x}^{p_{1}, \infty}(X)}\|f\|_{L^{p_{1}, 1}(X)} .
\end{aligned}
$$

Afin de faciliter la lecture de la preuve de l'assertion b), nous donnons un autre argument qui utilise (70) et la norme $\|\cdot\|_{L^{p_{1}, \infty}(X)}$ de $L^{p_{1}, \infty}(X)$, définie en (65), car elle présente l'avantage de satisfaire l'inégalité triangulaire. En effet, on a

$$
\begin{aligned}
\left\|P_{n} f\right\|_{L^{p_{1}, \infty}(X)} & \leq \int_{X}\left\|K_{n}\left(x, x^{\prime}\right)\right\| \|_{L_{x}^{p_{1}, \infty}(X)}\left|f\left(x^{\prime}\right)\right| d \mu\left(x^{\prime}\right) \\
& \leq\|\| K_{n}\left(x, x^{\prime}\right)\left\|_{L_{x}^{p_{1}, \infty}(X)}\right\|_{L_{x^{\prime}}^{q_{1}, \infty}(X)}\|f\|_{L^{p_{1}, 1}(X)} .
\end{aligned}
$$

Les deux arguments précédents sont en fait duaux. En utilisant (69) ou (71), on obtient de même la bornitude de l'opérateur

$$
P_{n}: L_{x}^{p_{2}, 1}(X) \rightarrow L_{x}^{p_{2}, \infty}(X) .
$$

On conclut par interpolation réelle (à savoir le théorème 2.32 avec $B=\mathbb{C}$ ).

b) On affirme que l'opérateur suivant est borné

$$
\oplus P_{n}: L_{x}^{p_{1}, 1}\left(X, \ell^{\infty}(\mathbb{N})\right) \rightarrow L_{x}^{p_{1}, \infty}\left(X, \ell^{\infty}(\mathbb{N})\right) .
$$

En effet, cela découle de l'inégalité de Hölder (66) et de (68) :

$$
\begin{aligned}
\sup _{n \in \mathbb{N}}\left|\left(P_{n} f_{n}\right)(x)\right| & =\sup _{n \in \mathbb{N}}\left|\int_{X} K_{n}\left(x, x^{\prime}\right) f_{n}\left(x^{\prime}\right) d \mu\left(x^{\prime}\right)\right| \\
& \leq \sup _{n \in \mathbb{N}} \int_{X}\left|K_{n}\left(x, x^{\prime}\right)\right| \sup _{m \in \mathbb{N}}\left|f_{m}\left(x^{\prime}\right)\right| d \mu\left(x^{\prime}\right) \\
& \leq \underbrace{\left(\sup _{n \in \mathbb{N}}\left\|K_{n}\left(x, x^{\prime}\right)\right\|_{L_{x^{\prime}}^{q_{1}, \infty}(X)}\right)}_{\in L_{x}^{p_{1}}, \infty} \times\left\|\sup _{m \in \mathbb{N}}\left|f_{m}\left(x^{\prime}\right)\right|\right\|_{L_{x^{\prime}}^{p_{1}, 1}(X)}
\end{aligned}
$$

On s'attelle maintenant à prouver la bornitude de l'opérateur

$$
\oplus P_{n}: L_{x}^{p_{1}, 1}\left(X, \ell^{1}(\mathbb{N})\right) \rightarrow L_{x}^{p_{1}, \infty}\left(X, \ell^{1}(\mathbb{N})\right) .
$$


De nouveau, on utilise la norme $\|\cdot\|_{L^{p_{1}, \infty}(X)}$ de $L^{p_{1}, \infty}(X)$. L'hypothèse (70) et l'inégalité de Hölder (66) nous amènent aux estimations

$$
\begin{aligned}
\left\|\sum_{n \in \mathbb{N}}\left|\left(P_{n} f_{n}\right)(x)\right|\right\| \|_{L_{x}^{p_{1}, \infty}(X)} & =\left\|\sum_{n \geq 0}\left|\int_{X} K_{n}\left(x, x^{\prime}\right) f_{n}\left(x^{\prime}\right) d \mu\left(x^{\prime}\right)\right|\right\| \|_{L_{x}^{p_{1}, \infty}(X)} \\
& \leq \sum_{n \geq 0} \int_{X}\left\|K_{n}\left(x, x^{\prime}\right)\right\|_{L_{x}^{p_{1}, \infty}(X)}\left|f_{n}\left(x^{\prime}\right)\right| d \mu\left(x^{\prime}\right) \\
& \leq \sum_{n \geq 0} \int_{X}\left(\sup _{m \in \mathbb{N}}\left\|K_{m}\left(x, x^{\prime}\right)\right\|_{L_{x}^{p_{1}, \infty}(X)}\right)\left|f_{n}\left(x^{\prime}\right)\right| d \mu\left(x^{\prime}\right) \\
& \leq \int_{X}\left(\sup _{m \in \mathbb{N}}\left\|K_{m}\left(x, x^{\prime}\right)\right\|_{L_{x}^{p_{1}, \infty}(X)}\right)\left(\sum_{n \geq 0}\left|f_{n}\left(x^{\prime}\right)\right|\right) d \mu\left(x^{\prime}\right) \\
& \leq\left\|\sup _{m \in \mathbb{N}}\right\| K_{m}\left(x, x^{\prime}\right)\left\|_{L_{x}^{p_{1}, \infty}(X)}\right\|_{L_{x^{\prime}}^{q_{1}, \infty}(X)}\left\|\sum_{n \geq 0}\left|f_{n}\left(x^{\prime}\right)\right|\right\|_{L_{x^{\prime}}^{p_{1}, 1}(X)} .
\end{aligned}
$$

La démonstration est évidemment similaire en remplaçant $p_{1}$ par $p_{2}$ et en utilisant (71) et (69). On a ainsi obtenu la bornitude des quatre opérateurs

$$
\begin{aligned}
& \oplus P_{n}: L_{x}^{p_{1}, 1}\left(X, \ell^{\infty}(\mathbb{N})\right) \rightarrow L_{x}^{p_{1}, \infty}\left(X, \ell^{\infty}(\mathbb{N})\right), \\
& \oplus P_{n}: L_{x}^{p_{2}, 1}\left(X, \ell^{\infty}(\mathbb{N})\right) \rightarrow L_{x}^{p_{2}, \infty}\left(X, \ell^{\infty}(\mathbb{N})\right), \\
& \oplus P_{n}: L_{x}^{p_{1}, 1}\left(X, \ell^{1}(\mathbb{N}) \rightarrow L_{x}^{p_{1}, \infty}\left(X, \ell^{1}(\mathbb{N})\right),\right. \\
& \oplus P_{n}: L_{x}^{p_{2}, 1}\left(X, \ell^{1}(\mathbb{N})\right) \rightarrow L_{x}^{p_{2}, \infty}\left(X, \ell^{1}(\mathbb{N})\right) .
\end{aligned}
$$

Pour tout $p \in] p_{1}, p_{2}[$, le théorème 2.32 assure la bornitude des deux opérateurs suivants par interpolation réelle

$$
\begin{aligned}
& \oplus P_{n}: L_{x}^{p}\left(X, \ell^{\infty}(\mathbb{N})\right) \rightarrow L_{x}^{p}\left(X, \ell^{\infty}(\mathbb{N})\right), \\
& \oplus P_{n}: L_{x}^{p}\left(X, \ell^{1}(\mathbb{N})\right) \rightarrow L_{x}^{p}\left(X, \ell^{1}(\mathbb{N})\right) .
\end{aligned}
$$

Par interpolation complexe (c'est-à-dire le théorème 2.26 avec $\theta=\frac{1}{2}$ ), on obtient la bornitude de l'opérateur

$$
\oplus P_{n}: L_{x}^{p}\left(X, \ell^{2}(\mathbb{N})\right) \rightarrow L_{x}^{p}\left(X, \ell^{2}(\mathbb{N})\right) .
$$

Il s'agit maintenant d'écrire un résultat analogue au lemme 2.33 adaptée à la théorie générale avec $\operatorname{dim}\left(E_{n}\right) \neq 1$. Pour cela, on aura besoin d'espaces analogues à $\ell^{1}(\mathbb{N})$ et $\ell^{\infty}(\mathbb{N})$ adaptés aux sous-espaces $E_{n}$, ce sera le rôle joué par les espaces $\left(\oplus E_{n}\right)_{\ell^{r}}$ dans la preuve du résultat suivant.

Lemme 2.34. - Fixons des réels $p_{1}<p<p_{2}$ appartenant à $] 1,+\infty\left[\right.$ et considérons $K_{n}: X^{2} \rightarrow \mathbb{C}$ des fonctions mesurables, pour $n \in \mathbb{N}$, de sorte que

$$
\begin{aligned}
& \sup _{n \geq 0}\left\|K_{n}\left(x, x^{\prime}\right)\right\|_{L_{x^{\prime}}^{q_{1}, \infty}(X)} \in L_{x}^{p_{1}, \infty}(X), \\
& \sup _{n \geq 0}\left\|K_{n}\left(x, x^{\prime}\right)\right\|_{L_{x^{\prime}}^{q_{2}, \infty}(X)} \in L_{x}^{p_{2}, \infty}(X), \\
& \sup _{n \geq 0}\left\|K_{n}\left(x, x^{\prime}\right)\right\|_{L_{x}^{p_{1}, \infty}(X)} \in L_{x^{\prime}}^{q_{1}, \infty}(X), \\
& \sup _{n \geq 0}\left\|K_{n}\left(x, x^{\prime}\right)\right\|_{L_{x}^{p_{2}, \infty}(X)} \in L_{x^{\prime}}^{q_{2}, \infty}(X) .
\end{aligned}
$$

Alors les deux assertions suivantes sont vraies

a) pour tout entier $n \in \mathbb{N}$, l'opérateur $P_{n}$ défini par

$$
\forall u \in L_{x}^{p}\left(X, E_{n}\right) \quad \forall(x, y) \in X^{2} \quad\left(P_{n} u\right)(x, y)=\int_{X} K_{n}\left(x, x^{\prime}\right) u\left(x^{\prime}, y\right) d \mu\left(x^{\prime}\right),
$$

est borné sur $L_{x}^{p}\left(X, E_{n}\right)$ et la borne supérieure $\sup _{n \geq 0}\left\|P_{n}\right\|$ est finie. 
b) l'opérateur $\oplus P_{n}$, défini par l'expression suivante, est borné

$$
\begin{aligned}
\oplus P_{n}: L_{x}^{p}\left(X, \oplus E_{n}\right) & \rightarrow L_{x}^{p}\left(X, \oplus E_{n}\right) \\
\left(u_{n}(x, y)\right)_{n \in \mathbb{N}} & \mapsto\left(\left(P_{n} u_{n}\right)(x, y)\right)_{n \in \mathbb{N}}
\end{aligned}
$$

Preuve. a) On raisonne comme pour le lemme 2.33, c'est-à-dire que chaque opérateur $P_{n}$ est borné de $L_{x}^{p_{1}, 1}\left(X, E_{n}\right)$ à valeurs dans $L_{x}^{p_{1}, \infty}\left(X, E_{n}\right)$ et de $L_{x}^{p_{2}, 1}\left(X, E_{n}\right)$ à valeurs dans $L_{x}^{p_{2}, \infty}\left(X, E_{n}\right)$ avec des normes majorées indépendamment de $n$. On conclut par interpolation réelle (c'est-à-dire le théorème 2.32 avec $\left.B=E_{n}\right)$.

b) L'idée est d'interpoler l'espace de Hilbert $\oplus E_{n}$ entre les deux espaces

$$
\begin{aligned}
\left(\oplus E_{n}\right)_{\ell^{1}} & :=\left\{\left(u_{n}\right)_{n \in \mathbb{N}}, \quad \forall n \in \mathbb{N}, u_{n} \in E_{n} ; \quad \sum_{n \in \mathbb{N}}\left\|u_{n}\right\|_{L_{y}^{2}(X)}<+\infty\right\}, \\
\left(\oplus E_{n}\right)_{\ell^{\infty}} & :=\left\{\left(u_{n}\right)_{n \in \mathbb{N}}, \quad \forall n \in \mathbb{N}, u_{n} \in E_{n} ; \quad \sup _{n \in \mathbb{N}}\left\|u_{n}\right\|_{L_{y}^{2}(X)}<+\infty\right\}
\end{aligned}
$$

qui sont munis de leurs normes naturelles. On vérifie aisément que les espaces $\left(\oplus E_{n}\right)_{\ell^{1}}$ et $\left(\oplus E_{n}\right)_{\ell^{\infty}}$ sont des rétractes, au sens de la définition 2.27 , des espaces $\ell^{1}\left(\mathbb{N}, \oplus E_{n}\right)$ et $\ell^{\infty}\left(\mathbb{N}, \oplus E_{n}\right)$. Par suite, le corollaire 2.30 et la théorie de l'interpolation complexe des espaces $\ell^{r}\left(\mathbb{N}, \oplus E_{n}\right)$ (c'est-à-dire le théorème 2.26) montrent que l'on a l'égalité

$$
\left[\left(\oplus E_{n}\right)_{\ell^{1}},\left(\oplus E_{n}\right)_{\ell^{\infty}}\right]_{\frac{1}{2}}=\oplus E_{n}
$$

avec équivalence de normes. Nous pouvons donc reprendre l'argumentation du lemme 2.33 et il nous suffit manifestement de prouver la bornitude des quatre opérateurs

$$
\begin{array}{lll}
\oplus P_{n}: L_{x}^{p_{1}, 1}\left(X,\left(\oplus E_{n}\right)_{\ell^{1}}\right) & \rightarrow & L_{x}^{p_{1}, \infty}\left(X,\left(\oplus E_{n}\right)_{\ell^{1}}\right), \\
\oplus P_{n}: L_{x}^{p_{2}, 1}\left(X,\left(\oplus E_{n}\right)_{\ell^{1}}\right) & \rightarrow & L_{x}^{p_{2}, \infty}\left(X,\left(\oplus E_{n}\right)_{\ell^{1}}\right), \\
\oplus P_{n}: L_{x}^{p_{1}, 1}\left(X,\left(\oplus E_{n}\right)_{\ell^{\infty}}\right) & \rightarrow L_{x}^{p_{1}, \infty}\left(X,\left(\oplus E_{n}\right)_{\ell^{\infty}}\right), \\
\oplus P_{n}: L_{x}^{p_{2}, 1}\left(X,\left(\oplus E_{n}\right)_{\ell^{\infty}}\right) & \rightarrow L_{x}^{p_{2}, \infty}\left(X,\left(\oplus E_{n}\right)_{\ell^{\infty}}\right) .
\end{array}
$$

Par symétrie évidente, il nous suffit de justifier la bornitude des deux opérateurs

$$
\begin{array}{lll}
\oplus P_{n}: L_{x}^{p_{1}, 1}\left(X,\left(\oplus E_{n}\right)_{\ell^{1}}\right) & \rightarrow & L_{x}^{p_{1}, \infty}\left(X,\left(\oplus E_{n}\right)_{\ell^{1}}\right) \\
\oplus P_{n}: L_{x}^{p_{1}, 1}\left(X,\left(\oplus E_{n}\right)_{\ell^{\infty}}\right) & \rightarrow & L_{x}^{p_{1}, \infty}\left(X,\left(\oplus E_{n}\right)_{\ell^{\infty}}\right) .
\end{array}
$$

L'hypothèse (74) et l'inégalité de Hölder (66) nous donnent les majorations

$$
\begin{aligned}
\|\| \sum_{n \geq 0} \| & \left(P_{n} u_{n}\right)(x, y)\left\|_{L_{y}^{2}(X)}\right\| \|_{L_{x}^{p_{1}, \infty}(X)} \\
& =\left\|\sum_{n \geq 0}\right\| \int_{X} K_{n}\left(x, x^{\prime}\right) u_{n}\left(x^{\prime}, y\right) d \mu\left(x^{\prime}\right)\|\|_{L_{y}^{2}(X)}\|\|_{L_{x}^{p_{1}, \infty}(X)} \\
& \leq \sum_{n \geq 0} \int_{X}\left\|K_{n}\left(x, x^{\prime}\right)\right\|_{L_{x}^{p_{1}, \infty}(X)}\left\|u_{n}\left(x^{\prime}, y\right)\right\|_{L_{y}^{2}(X)} d \mu\left(x^{\prime}\right) \\
& \leq \int_{X}\left(\sup _{m \in \mathbb{N}}\left\|K_{m}\left(x, x^{\prime}\right)\right\|_{L_{x}^{p_{1}, \infty}(X)}\right)\left(\sum_{n \geq 0}\left\|u_{n}\left(x^{\prime}, y\right)\right\|_{L_{y}^{2}(X)}\right) d \mu\left(x^{\prime}\right) \\
& \leq\left\|\sup _{m \in \mathbb{N}}\right\| K_{m}\left(x, x^{\prime}\right)\|\|_{L_{x}^{p_{1}, \infty}(X)}\|\|_{L_{x^{\prime}}^{q_{1}, \infty}(X)}\left\|\sum_{n \geq 0}\right\| u_{n}\left(x^{\prime}, y\right)\left\|_{L_{y}^{2}(X)}\right\|_{L_{x^{\prime}}^{p_{1}, 1}(X)}
\end{aligned}
$$


Enfin, (72) et l'inégalité de Hölder (66) nous permettent d'écrire

$$
\begin{aligned}
\sup _{n \in \mathbb{N}}\left\|\left(P_{n} u_{n}\right)(x, y)\right\|_{L_{y}^{2}(X)} & =\sup _{n \in \mathbb{N}}\left\|\int_{X} K_{n}\left(x, x^{\prime}\right) u_{n}\left(x^{\prime}, y\right) d \mu\left(x^{\prime}\right)\right\|_{L_{y}^{2}(X)} \\
& \leq \sup _{n \in \mathbb{N}} \int_{X}\left|K_{n}\left(x, x^{\prime}\right)\right| \sup _{m \in \mathbb{N}}\left\|u_{m}\left(x^{\prime}, y\right)\right\|_{L_{y}^{2}(X)} d \mu\left(x^{\prime}\right) \\
& \leq \underbrace{\left(\sup _{n \in \mathbb{N}}\left\|K_{n}\left(x, x^{\prime}\right)\right\|_{L_{x^{\prime}}^{q_{1}, \infty}(X)}\right)}_{\in L_{x}^{p_{1}, \infty}(X)} \times\left\|\sup _{m \in \mathbb{N}}\right\| u_{m}\left(x^{\prime}, y\right)\left\|_{L_{y}^{2}(X)}\right\|_{L_{x^{\prime}}^{p_{1}, 1}(X)}
\end{aligned}
$$

2.8. Preuve du théorème 2.6 : bornitude de $S_{p} R_{p}$. - Sous les hypothèses du théorème 2.6 de dualité, les exposants $p_{1}$ et $p_{2}$ sont conjugués et l'on souhaite montrer que $S_{p} R_{p}$ est un projecteur borné $\operatorname{sur} L_{x}^{p}\left(X, \oplus E_{n}\right)$ pour tout $\left.p \in\right] p_{1}, p_{2}\left[\right.$. Avec les notations usuelles, on a $q_{1}=\frac{p_{1}}{p_{1}-1}=p_{2}$ et $q_{2}=\frac{p_{2}}{p_{2}-1}=p_{1}$. D'après la forme du projecteur (60), il s'agit d'appliquer le lemme 2.34 avec la suite de noyaux $K_{n}$ définis par

$$
\forall\left(x, x^{\prime}\right) \in X^{2} \quad K_{n}\left(x, x^{\prime}\right)=\frac{1}{d_{n}} \sqrt{e_{n}(x) e_{n}\left(x^{\prime}\right)} .
$$

Il est facile de constater que (35) et (37) impliquent l'assertion (72) (qui est identique à (75)). En effet, on a pour tout $(x, n) \in X \times \mathbb{N}$

$$
\begin{aligned}
\left\|\frac{1}{d_{n}} \sqrt{e_{n}(x) e_{n}\left(x^{\prime}\right)}\right\|_{L_{x^{\prime}}^{q_{1}, \infty}(X)} & \leq \frac{\sqrt{e_{n}(x)}}{d_{n}}\left\|\sqrt{e_{n}}\right\|_{L^{q_{1}}(X)} \\
& \leq \frac{\sqrt{e_{n}(x)}}{\left\|\sqrt{e_{n}}\right\|_{L^{p_{1}}(X)}} \times \frac{\left\|\sqrt{e_{n}}\right\|_{L^{p_{1}}(X)}\left\|\sqrt{e_{n}}\right\|_{L^{q_{1}}(X)}}{d_{n}} .
\end{aligned}
$$

Les deux autres hypothèses (73) et (74) du lemme 2.34 se vérifient de la même façon. La preuve du théorème 2.6 de dualité est finie.

2.9. Preuve du théorème 2.5 : défaut d'interpolation. - Nous allons définir la notion de défaut d'interpolation afin d'aborder l'interpolation des espaces $\mathbf{P} \mathbf{L}^{p}\left(X, \oplus E_{n}\right)$. Commençons par introduire quelques notations. Pour tous réels $p_{1}, p$ et $p_{2}$ appartenant à $\left[1,+\infty\right.$ [ et vérifiant $p_{1} \leq p \leq p_{2}$, on définit les nombres $\theta_{1}\left(p_{1}, p, p_{2}\right)$ et $\theta_{2}\left(p_{1}, p, p_{2}\right)$ tels que

$$
\frac{\theta_{1}\left(p_{1}, p, p_{2}\right)}{p_{1}}+\frac{\theta_{2}\left(p_{1}, p, p_{2}\right)}{p_{2}}=\frac{1}{p} \quad \text { et } \quad \theta_{1}\left(p_{1}, p, p_{2}\right)+\theta_{2}\left(p_{1}, p, p_{2}\right)=1 .
$$

De façon précise, on a les formules

$$
\theta_{1}\left(p_{1}, p, p_{2}\right)=\frac{\frac{1}{p}-\frac{1}{p_{2}}}{\frac{1}{p_{1}}-\frac{1}{p_{2}}} \quad \text { et } \quad \theta_{2}\left(p_{1}, p, p_{2}\right)=\frac{\frac{1}{p_{1}}-\frac{1}{p}}{\frac{1}{p_{1}}-\frac{1}{p_{2}}} .
$$

On peut alors poser la définition suivante.

Définition 2.35. - Considérons $p_{1}$ et $p_{2}$ appartenant à $\left[1,+\infty\left[\right.\right.$ et vérifiant $p_{1}<p_{2}$ ainsi qu'une fonction non nulle $\phi \in L^{p_{1}}(X) \cap L^{p_{2}}(X)$. Nous définissons $Q\left(\phi,\left[p_{1}, p_{2}\right]\right) \in[1,+\infty[$ le défaut d'interpolation de $\phi$ sur $\left[p_{1}, p_{2}\right]$ par la formule

$$
Q\left(\phi,\left[p_{1}, p_{2}\right]\right):=\sup _{p \in\left[p_{1}, p_{2}\right]} \frac{\|\phi\|_{L^{p_{1}}(X)}^{\theta_{1}\left(p_{1}, p, p_{2}\right)}\|\phi\|_{L^{p_{2}}(X)}^{\theta_{2}\left(p_{1}, p, p_{2}\right)}}{\|\phi\|_{L^{p}(X)}} .
$$


L'inégalité $Q\left(\phi,\left[p_{1}, p_{2}\right]\right)<+\infty$ est facile et nous allons la vérifier par convexité (voir le lemme 2.36 ciaprès). Quant à l'inégalité $Q\left(\phi,\left[p_{1}, p_{2}\right]\right) \geq 1$, elle découle de l'inégalité de Hölder avec les exposants conjugués $\frac{p_{1}}{p \theta_{1}\left(p_{1}, p, p_{2}\right)}$ et $\frac{p_{2}}{p \theta_{2}\left(p_{1}, p, p_{2}\right)}$ :

$$
\begin{aligned}
\left(\int_{X}|\phi(x)|^{p} d \mu(x)\right)^{\frac{1}{p}} & =\left(\int_{X}|\phi(x)|^{p \theta_{1}\left(p_{1}, p, p_{2}\right)}|\phi(x)|^{p \theta_{2}\left(p_{2}, p, p_{2}\right)} d \mu(x)\right)^{\frac{1}{p}} \\
& \leq\left(\int_{X}|\phi(x)|^{p_{1}} d \mu(x)\right)^{\frac{\theta_{1}\left(p_{1}, p, p_{2}\right)}{p_{1}}}\left(\int_{X}|\phi(x)|^{p_{2}} d \mu(x)\right)^{\frac{\theta_{2}\left(p_{1}, p, p_{2}\right)}{p_{2}}} .
\end{aligned}
$$

La condition d'égalité de l'inégalité de Hölder montre alors l'équivalence

$$
Q\left(\phi,\left[p_{1}, p_{2}\right]\right)=1 \quad \Leftrightarrow \quad \exists a>0 \quad \exists A \in \mathcal{B}(X), \quad 0<\mu(A)<+\infty, \quad|\phi|=a \mathbf{1}_{A},
$$

où $\mathcal{B}(X)$ est l'ensemble des parties mesurables de $X$. Ainsi, le défaut d'interpolation de la fonction $\phi$ permet de tester si elle se concentre complètement sur une même partie de l'espace mesuré $X$. Par comparaison avec (76), le défaut d'interpolation permet de minorer $\|\phi\|_{L^{p}(X)}$ si l'on connaît $\|\phi\|_{L^{p_{1}}(X)}$ et $\|\phi\|_{L^{p_{2}(X)}}$ :

$$
\forall p \in\left[p_{1}, p_{2}\right] \quad \frac{\|\phi\|_{L^{p_{1}}(X)}^{\theta_{1}\left(p_{1}, p, p_{2}\right)}\|\phi\|_{L_{2}^{p}(X)}^{\theta_{2}\left(p_{1}, p, p_{2}\right)}}{Q\left(\phi,\left[p_{1}, p_{2}\right]\right)} \leq\|\phi\|_{L^{p}(X)} .
$$

Le lemme suivant montre qu'il suffit d'examiner un seul point de l'intervalle $] p_{1}, p_{2}\left[\right.$ pour contrôler $Q\left(\phi,\left[p_{1}, p_{2}\right]\right)$.

Lemme 2.36. - Fixons des réels $p_{1}<p<p_{2}$ appartenant à $[1,+\infty[$ et une suite de fonctions non nulles $\left(\phi_{n}\right)_{n \in \mathbb{N}}$ de $L^{p_{1}}(X) \cap L^{p_{2}}(X)$, alors on a l'équivalence :

$$
\sup _{n \in \mathbb{N}} \frac{\left\|\phi_{n}\right\|_{L^{p_{1}}(X)}^{\theta_{1}\left(p_{1}, p, p_{2}\right)}\left\|\phi_{n}\right\|_{L^{p_{2}(X)}}^{\theta_{2}\left(p_{1}, p, p_{2}\right)}}{\left\|\phi_{n}\right\|_{L^{p}(X)}}<+\infty \quad \Leftrightarrow \quad \sup _{n \in \mathbb{N}} Q\left(\phi_{n},\left[p_{1}, p_{2}\right]\right)<+\infty .
$$

Preuve. Pour toute fonction non nulle $\phi \in L^{p_{1}}(X) \cap L^{p_{2}}(X)$, il est bien connu que la fonction

$$
\Phi: \wp \in\left[p_{1}, p_{2}\right] \mapsto \ln \left(\|\phi\|_{L^{\wp}(X)}\right)
$$

est convexe par rapport à $\frac{1}{\wp}$ (voir les inégalités (76)). Introduisons la fonction $\widetilde{\Phi}:\left[p_{1}, p_{2}\right] \rightarrow \mathbb{R}$ définie par

$$
\widetilde{\Phi}(\wp)=\ln \left(\frac{\|\phi\|_{L^{p_{1}}(X)}^{\theta_{1}\left(p_{1}, \wp, p_{2}\right)}\|\phi\|_{L^{p_{2}}(X)}^{\theta_{2}\left(p_{1}, \wp, p_{2}\right)}}{\|\phi\|_{L^{\wp}(X)}}\right)=\theta_{1}\left(p_{1}, \wp, p_{2}\right) \Phi\left(p_{1}\right)+\theta_{2}\left(p_{1}, \wp, p_{2}\right) \Phi\left(p_{2}\right)-\Phi(\wp) .
$$

La fonction $\widetilde{\Phi}$ s'annule en $p_{1}$ et $p_{2}$ et est concave par rapport à $\frac{1}{\wp}$.

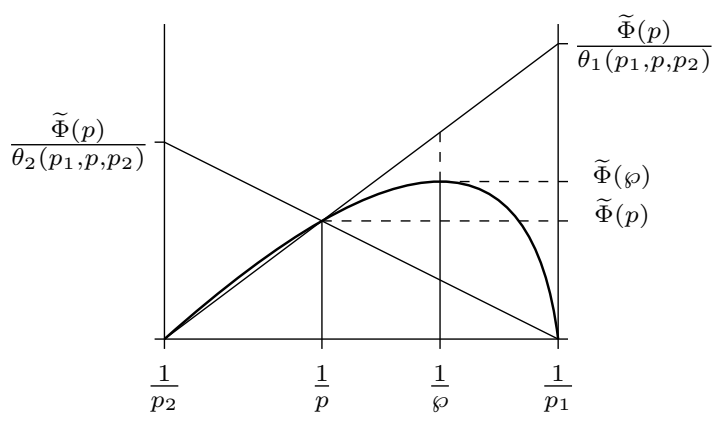

Par concavité et application du théorème de Thalès, nous avons les estimations

$$
\forall \wp \in\left[p_{1}, p_{2}\right] \quad \widetilde{\Phi}(\wp) \leq \widetilde{\Phi}(p) \max \left(\frac{\frac{1}{p_{1}}-\frac{1}{p_{2}}}{\frac{1}{p}-\frac{1}{p_{2}}}, \frac{\frac{1}{p_{1}}-\frac{1}{p_{2}}}{\frac{1}{p_{1}}-\frac{1}{p}}\right) .
$$


En passant à l'exponentielle, on obtient les estimations suivantes qui donnent la conclusion :

$$
\frac{\|\phi\|_{L^{p_{1}}(X)}^{\theta_{1}\left(p_{1}, p, p_{2}\right)}\|\phi\|_{L^{p_{2}(X)}}^{\theta_{2}\left(p_{1}, p, p_{2}\right)}}{\|\phi\|_{L^{p}(X)}} \leq Q\left(\phi,\left[p_{1}, p_{2}\right]\right) \leq\left(\frac{\|\phi\|_{L^{p_{1}}(X)}^{\theta_{1}\left(p_{1}, p, p_{2}\right)}\|\phi\|_{L^{p_{2}}(X)}^{\theta_{2}\left(p_{1}, p, p_{2}\right)}}{\|\phi\|_{L^{p}(X)}}\right)^{C\left(p_{1}, p, p_{2}\right)} .
$$

On sait que pour tout réel $p \in] 1,+\infty\left[\right.$ et toute fonction $\phi \in L^{p}(X)$, il existe une fonction $\psi \in L^{\frac{p}{p-1}}(X)$ telle que

$$
\int_{X} \phi(x) \psi(x) d \mu(x)=\|\phi\|_{L^{p}(X)}\|\psi\|_{L^{\frac{p}{p-1}}(X)} .
$$

Par exemple, si $\phi$ est positive alors on peut choisir $\psi(x)=\phi(x)^{p-1}$. Le défaut d'interpolation permet de formuler des propriétés analogues si $\phi$ appartient à deux espaces de Lebesgue $L^{p_{1}}(X)$ et $L^{p_{2}}(X)$.

Proposition 2.37. - Fixons $p_{1}$ et $p_{2}$ appartenant à $] 1,+\infty\left[\right.$ et vérifiant $p_{1}<p_{2}$ et $\frac{1}{p_{1}}+\frac{1}{p_{2}} \leq 1$. Il existe un réel $r=r\left(p_{1}, p_{2}\right)>1$ tel que pour toute fonction non nulle $\phi \in L^{p_{1}}(X) \cap L^{p_{2}}(X)$ on peut trouver une fonction $\psi \in L^{q_{2}}(X) \cap L^{q_{1}}(X)$, avec $q_{2}=\frac{p_{2}}{p_{2}-1}$ et $q_{1}=\frac{p_{1}}{p_{1}-1}$, de sorte que l'on a

$$
\begin{aligned}
\int_{X} \phi(x) \psi(x) d \mu(x) & =1 \\
\|\phi\|_{L^{p_{1}(X)}}\|\psi\|_{L^{q_{1}}(X)} & \leq Q\left(\phi,\left[p_{1}, p_{2}\right]\right)^{r}, \\
\|\phi\|_{L^{p_{2}(X)}}\|\psi\|_{L^{q_{2}(X)}} & \leq Q\left(\phi,\left[p_{1}, p_{2}\right]\right)^{r}, \\
\frac{|\psi|^{q_{1}}}{\int_{X}|\psi(x)|^{q_{1}} d \mu(x)} & \leq Q\left(\phi,\left[p_{1}, p_{2}\right]\right)^{(r-1) q_{1}}\left[\frac{|\phi|^{p_{1}}}{\int_{X}|\phi(x)|^{p_{1}} d \mu(x)}+\frac{|\phi|^{p_{2}}}{\int_{X}|\phi(x)|^{p_{2}} d \mu(x)}\right], \\
\frac{|\psi|^{q_{2}}}{\int_{X}|\psi(x)|^{q_{2}} d \mu(x)} & \leq Q\left(\phi,\left[p_{1}, p_{2}\right]\right)^{(r-1) q_{2}}\left[\frac{|\phi|^{p_{1}}}{\int_{X}|\phi(x)|^{p_{1}} d \mu(x)}+\frac{|\phi|^{p_{2}}}{\int_{X}|\phi(x)|^{p_{2}} d \mu(x)}\right] .
\end{aligned}
$$

Preuve. Commençons par le calcul élémentaire suivant :

$$
p_{2} q_{2}-p_{1} q_{1}=\frac{p_{2}^{2}}{p_{2}-1}-\frac{p_{1}^{2}}{p_{1}-1}=\frac{p_{2}^{2}\left(p_{1}-1\right)-p_{1}^{2}\left(p_{2}-1\right)}{\left(p_{2}-1\right)\left(p_{1}-1\right)}=\frac{\left(p_{2}-p_{1}\right)\left(p_{1} p_{2}-p_{1}-p_{2}\right)}{\left(p_{2}-1\right)\left(p_{1}-1\right)} .
$$

Par conséquent on a $p_{2} q_{2} \geq p_{1} q_{1}$ et l'on peut choisir un réel $r \in\left[1+\frac{p_{1}}{q_{2}}, 1+\frac{p_{2}}{q_{1}}\right]$. En particulier, on a $r \in\left[p_{1}, p_{2}\right]$ et

$$
p_{1} \leq(r-1) q_{2} \leq(r-1) q_{1} \leq p_{2} .
$$

On peut maintenant considérer la fonction $\psi$ définie par

$$
\forall x \in X \quad \psi(x)=\left\{\begin{array}{cr}
\frac{1}{\int_{X}\left|\phi\left(x^{\prime}\right)\right|^{r} d \mu\left(x^{\prime}\right)} \times \frac{|\phi(x)|^{r}}{\phi(x)} & \text { si } \phi(x) \neq 0 \\
0 & \text { si } \phi(x)=0 .
\end{array}\right.
$$

La définition précédente est licite car, d'après (76), la fonction $\phi$ appartient à $L^{p}(X)$ pour tout $p \in\left[p_{1}, p_{2}\right]$. Ensuite, il est clair que la fonction $\phi \psi$ est positive est d'intégrale égale à 1 . Les inégalités (78) impliquent que $\psi$ appartient à $L^{q_{2}}(X) \cap L^{q_{1}}(X)$. Grâce à (76) et (77), il vient

$$
\|\phi\|_{L^{p_{1}(X)}}\|\psi\|_{L^{q_{1}(X)}}=\frac{\|\phi\|_{L^{p_{1}}(X)}\|\phi\|_{L^{(r-1) q_{1}}(X)}^{r-1}}{\|\phi\|_{L^{r}(X)}^{r}} \leq Q\left(\phi,\left[p_{1}, p_{2}\right]\right)^{r}\|\phi\|_{L^{p_{1}(X)}}^{\alpha}\|\phi\|_{L^{p_{2}(X)}}^{\beta},
$$

où l'on a noté

$$
\begin{aligned}
\alpha & :=1+(r-1) \theta_{1}\left(p_{1},(r-1) q_{1}, p_{2}\right)-r \theta_{1}\left(p_{1}, r, p_{2}\right), \\
\beta & :=(r-1) \theta_{2}\left(p_{1},(r-1) q_{1}, p_{2}\right)-r \theta_{2}\left(p_{1}, r, p_{2}\right) .
\end{aligned}
$$

L'égalité $\theta_{1}+\theta_{2}=1$ implique que l'on a $\alpha+\beta=0$. En fait, il s'avère que $\alpha=\beta=0$. Cela peut se voir par calcul, mais puisque $\alpha$ ne dépend que de $\left(p_{1}, p_{2}, r\right)$, il suffit de traiter le cas particulier $X=\mathbb{R}$ muni de 
la mesure de Lebesgue. Pour tout $t>0$, si l'on pose $\phi_{t}=\mathbf{1}_{[0, t]}$ alors on a $\psi_{t}=\frac{1}{t} \mathbf{1}_{[0, t]}$ et $\left\|\phi_{t}\right\|_{L^{p}}=t^{\frac{1}{p}}$ pour tout $p \in[1,+\infty[$ et donc

$$
\left\|\phi_{t}\right\|_{L^{p_{1}(X)}}\left\|\psi_{t}\right\|_{L^{q_{1}(X)}}=t^{\frac{1}{p_{1}}+\frac{1}{q_{1}}-1}=1 \quad \text { et } \quad Q\left(\phi_{t},\left[p_{1}, p_{2}\right]\right)=1 .
$$

L'inégalité (79) devient

$$
\forall t>0 \quad 1 \leq t^{\alpha\left(\frac{1}{p_{1}}-\frac{1}{p_{2}}\right)} .
$$

Cela force les égalités $\alpha=\beta=0$. Un argument similaire permet d'estimer $\|\phi\|_{L^{p_{2}(X)}}\|\psi\|_{L^{q_{2}}(X)}$.

Passons aux estimations de $|\psi|^{q}$ avec $q \in\left\{q_{1}, q_{2}\right\}$. On se permet de noter $\theta_{1}=\theta_{1}\left(p_{1},(r-1) q, p_{2}\right)$ et $\theta_{2}=\theta_{2}\left(p_{1},(r-1) q, p_{2}\right)$. On obtient alors pour tout $x \in X$

$$
\frac{|\psi(x)|^{q}}{\|\psi\|_{L^{q}(X)}^{q}}=\frac{|\phi(x)|^{(r-1) q}}{\|\phi\|_{L^{(r-1) q}(X)}^{(r-1) q}} \leq Q\left(\phi,\left[p_{1}, p_{2}\right]\right)^{(r-1) q} \frac{|\phi(x)|^{(r-1) q}}{\|\phi\|_{L^{p_{1}(X)}}^{(r-1) q \theta_{1}}\|\phi\|_{L^{p_{2}(X)}}^{(r-1) q \theta_{2}}} .
$$

Les égalités $(r-1) q \theta_{1}+(r-1) q \theta_{2}=(r-1) q$ et $\frac{(r-1) q \theta_{1}}{p_{1}}+\frac{(r-1) q \theta_{2}}{p_{2}}=1$ nous donnent la conclusion

$$
\begin{aligned}
& \frac{|\psi(x)|^{q}}{\|\psi\|_{L^{q}(X)}^{q}} \leq Q\left(\phi,\left[p_{1}, p_{2}\right]\right)^{(r-1) q}\left(\frac{|\phi(x)|^{p_{1}}}{\|\phi\|_{L^{p_{1}(X)}}^{p_{1}}}\right)^{\frac{(r-1) q \theta_{1}}{p_{1}}}\left(\frac{|\phi(x)|^{p_{2}}}{\|\phi\|_{L^{p_{2}(X)}}^{p_{2}}}\right)^{\frac{(r-1) q \theta_{2}}{p_{2}}} \\
& \leq Q\left(\phi,\left[p_{1}, p_{2}\right]\right)^{(r-1) q}\left(\frac{(r-1) q \theta_{1}}{p_{1}} \frac{|\phi(x)|^{p_{1}}}{\|\phi\|_{L^{p_{1}(X)}}^{p_{1}}}+\frac{(r-1) q \theta_{2}}{p_{2}} \frac{|\phi(x)|^{p_{2}}}{\|\phi\|_{L^{p_{2}(X)}}^{p_{2}}}\right) \\
& \leq Q\left(\phi,\left[p_{1}, p_{2}\right]\right)^{(r-1) q}\left(\frac{|\phi(x)|^{p_{1}}}{\|\phi\|_{L^{p_{1}(X)}}^{p_{1}}}+\frac{|\phi(x)|^{p_{2}}}{\|\phi\|_{L^{p_{2}(X)}}^{p_{2}}}\right) .
\end{aligned}
$$

2.10. Preuve du théorème 2.5 : bornitude de $S_{p} R_{p, \psi}$ - - Nous avons maintenant les moyens d'achever la preuve, expliquée dans la partie 2.5, du théorème 2.5. Sous les hypothèses (35) et (36), il s'agit de justifier l'existence d'une suite $\left(\psi_{n}\right)_{n \in \mathbb{N}}$ de $L^{\frac{p_{2}}{p_{2}-1}}(X) \cap L^{\frac{p_{1}}{p_{1}-1}}(X)$ telle que

$$
\forall n \in \mathbb{N} \quad \int_{X} \frac{\sqrt{e_{n}(x)}}{\sqrt{d_{n}}} \psi_{n}(x) d \mu(x)=1,
$$

et que l'opérateur $S_{p} R_{p, \psi}$ soit borné sur $L_{x}^{p}\left(X, \oplus E_{n}\right)$ (voir (64)). L'hypothèse (36) et le lemme 2.36 nous apprennent que la suite des défauts d'interpolation $\left(Q\left(\sqrt{e_{n}},\left[p_{1}, p_{2}\right]\right)\right)_{n \in \mathbb{N}}$ est bornée. Par homogénéité, on a aussi

$$
\sup _{n \in \mathbb{N}} Q\left(\frac{\sqrt{e_{n}}}{\sqrt{d_{n}}},\left[p_{1}, p_{2}\right]\right)<+\infty \text {. }
$$

Par suite, la proposition 2.37 nous assure l'existence d'une constante $K>0$ et d'une suite de fonctions $\left(\psi_{n}\right)_{n \in \mathbb{N}}$ de $L^{\frac{p_{2}}{p_{2}-1}}(X) \cap L^{\frac{p_{1}}{p_{1}-1}}(X)$ qui vérifient (80) et les estimations suivantes uniformément en $n$ :

$$
\begin{aligned}
& \left\|\frac{\sqrt{e_{n}}}{\sqrt{d_{n}}}\right\|_{L^{p_{1}}(X)}\left\|\psi_{n}\right\|_{L^{q_{1}}(X)} \leq K, \\
& \left\|\frac{\sqrt{e_{n}}}{\sqrt{d_{n}}}\right\|_{L^{p_{2}(X)}}\left\|\psi_{n}\right\|_{L^{q_{2}}(X)} \leq K,
\end{aligned}
$$

ainsi que les inégalités suivantes pour tout $x \in X$

$$
\left(\frac{\left|\psi_{n}(x)\right|}{\left\|\psi_{n}\right\|_{L^{q_{1}(X)}}}\right)^{q_{1}}+\left(\frac{\left|\psi_{n}(x)\right|}{\left\|\psi_{n}\right\|_{L^{q_{2}(X)}}}\right)^{q_{2}} \leq K\left[\left(\frac{\sqrt{e_{n}(x)}}{\left\|\sqrt{e_{n}}\right\|_{L^{p_{1}(X)}}}\right)^{p_{1}}+\left(\frac{\sqrt{e_{n}(x)}}{\left\|\sqrt{e_{n}}\right\|_{L^{p_{2}(X)}}}\right)^{p_{2}}\right] .
$$

On va appliquer le lemme 2.34 avec les noyaux $K_{n}$ définis par

$$
\forall n \in \mathbb{N} \quad \forall\left(x, x^{\prime}\right) \in X^{2} \quad K_{n}\left(x, x^{\prime}\right):=\frac{\sqrt{e_{n}(x)}}{\sqrt{d_{n}}} \psi_{n}\left(x^{\prime}\right) .
$$


Les propriétés (72) et (73) se traitent comme pour la bornitude de $S_{p} R_{p}$. Par exemple, l'inclusion continue $L^{q_{1}}(X) \subset L^{q_{1}, \infty}(X)$ nous permet d'écrire pour tout $x \in X$

$$
\begin{aligned}
\left\|K_{n}\left(x, x^{\prime}\right)\right\|_{L_{x^{\prime}}^{q_{1}, \infty}(X)} & \leq \frac{\sqrt{e_{n}(x)}}{\sqrt{d_{n}}}\left\|\psi_{n}\right\|_{L^{q_{1}}(X)} \\
& \leq K \frac{\sqrt{e_{n}(x)}}{\left\|\sqrt{e_{n}}\right\|_{L^{p_{1}}(X)}} .
\end{aligned}
$$

L'hypothèse (35) implique alors (72). On montre de même (73). L'intérêt de la proposition 2.37 apparait pour démontrer (74) et (75). En effet, on a

$$
\begin{aligned}
\forall x^{\prime} \in X \quad\left\|K_{n}\left(x, x^{\prime}\right)\right\|_{L_{x}^{p_{1}, \infty}(X)} & \leq\left|\psi_{n}\left(x^{\prime}\right)\right|\left\|\frac{\sqrt{e_{n}}}{\sqrt{d_{n}}}\right\|_{L^{p_{1}}(X)} \\
& \leq K \frac{\left|\psi_{n}\left(x^{\prime}\right)\right|}{\left\|\psi_{n}\right\|_{L^{q_{1}}(X)}} .
\end{aligned}
$$

D'après (35) et (81), on obtient

$$
\left(\sup _{n \in \mathbb{N}}\left\|K_{n}\left(x, x^{\prime}\right)\right\|_{L_{x}^{p_{1}, \infty}(X)}\right)^{q_{1}} \in L_{x^{\prime}}^{1, \infty}(X)
$$

c'est-à-dire

$$
\sup _{n \in \mathbb{N}}\left\|K_{n}\left(x, x^{\prime}\right)\right\|_{L_{x}^{p_{1}, \infty}(X)} \in L_{x^{\prime}}^{q_{1}, \infty}(X)
$$

On a ainsi obtenu (74). Et (75) se traite de même. On a validé toutes les hypothèses du lemme 2.34 et l'on peut donc conclure que $S_{p} R_{p, \psi}$ est borné sur $L_{x}^{p}\left(X, \oplus E_{n}\right)$. Cela achève la preuve du théorème 2.5 d'interpolation.

\section{Espaces $\mathbf{P L}^{p}$ pour les harmoniques sphériques}

3.1. Reformulation des énoncés. - On reprend les notations de la partie 1.2. Commençons par remarquer que l'on peut naturellement identifier une somme d'une série $\sum a_{n} Z_{n}$ comme une distribution sur $\mathbb{S}^{d}$ dès lors que $\left(a_{n}\right)_{n \geq 1}$ est à croissance polynomiale. En effet, pour toute fonction test $\psi \in \mathcal{C}^{\infty}\left(\mathbb{S}^{d}\right)$ on a pour un paramètre $\varsigma \gg 1$ :

$$
\sum_{n \geq 1}\left|a_{n}\left\langle Z_{n}, \psi\right\rangle\right| \leq \sqrt{\sum_{n \geq 1}\left|a_{n}\right|^{2} n^{-2 \varsigma}} \sqrt{\sum_{n \geq 1} n^{2 \varsigma}\left|\left\langle Z_{n}, \psi\right\rangle\right|^{2}} .
$$

La série $\sum_{n \geq 1}\left|a_{n}\right|^{2} n^{-2 \varsigma}$ converge trivialement. Il en est de même de la seconde en utilisant la relation $(I-\Delta)^{\varsigma / 2} Z_{n}=(1+n(n+d-1))^{\varsigma / 2} Z_{n}$ et en faisant intervenir les espaces de Sobolev :

$$
\begin{aligned}
\sum_{n \geq 1} n^{2 \varsigma}\left|\left\langle Z_{n}, \psi\right\rangle\right|^{2} & \lesssim_{\varsigma, d} \sum_{n \geq 1}\left|\left\langle(I-\Delta)^{\varsigma / 2} Z_{n}, \psi\right\rangle\right|^{2} \\
& \lesssim_{\varsigma, d} \sum_{n \geq 1}\left|\left\langle Z_{n},(I-\Delta)^{\varsigma / 2} \psi\right\rangle\right|^{2} \\
& \lesssim_{\varsigma, d}\left\|(I-\Delta)^{\varsigma / 2} \psi\right\|_{L^{2}\left(\mathbb{S}^{d}\right)}:=\|\psi\|_{H^{\varsigma}\left(\mathbb{S}^{d}\right)}<+\infty .
\end{aligned}
$$

On peut donc naturellement identifier la suite $\left(a_{n} Z_{n}\right)$ à la distribution $\sum_{n \geq 1} a_{n} Z_{n}$. Ainsi, $\mathbf{P} \mathbf{L}^{p}\left(\mathbb{S}^{d}, \oplus \mathbb{C} Z_{n}\right)$ sera vu comme un espace de distributions sur $\mathbb{S}^{d}$. Une injection de Sobolev est une inclusion de la forme $H^{s} \subset L^{p}$, il est donc légitime de définir une injection de Sobolev probabiliste comme une inclusion de la forme $H^{s} \subset \mathbf{P L}^{p}$. Nous pouvons maintenant énoncer le résultat suivant (qui implique le théorème 1.1).

Proposition 3.1. - On considère une suite complexe $\left(a_{n}\right)_{n \geq 1}$ à croissance polynomiale. Pour tout réel $p \in] \frac{2 d}{d-1},+\infty\left[\right.$, la distribution $\sum_{n \geq 1} a_{n} Z_{n}$ appartient à $\mathbf{P} \mathbf{L}^{p}\left(\mathbb{S}^{d}, \oplus \mathbb{C} Z_{n}\right)$ si et seulement si l'on a

$$
\sum_{n \geq 1} \frac{1}{n^{d+1}}\left(\sum_{k=1}^{n} k^{d-1}\left|a_{k}\right|^{2}\right)^{\frac{p}{2}}<+\infty .
$$


Les espaces $\mathbf{P} \mathbf{L}^{p}\left(\mathbb{S}^{d}, \oplus \mathbb{C} Z_{n}\right)$ sont stables par interpolation réelle et complexe pour p parcourant $] \frac{2 d}{d-1},+\infty[$ au sens du théorème 2.5. Enfin, les injections de Sobolev probabilistes des fonctions $Z_{n}$ sont données par les inclusions

$$
H_{z o n}^{\frac{d-1}{2}-\frac{d}{p}}\left(\mathbb{S}^{d}\right) \subset \mathbf{P} \mathbf{L}^{p}\left(\mathbb{S}^{d}, \oplus \mathbb{C} Z_{n}\right) \subset \bigcap_{\varepsilon>0} H_{z o n}^{\frac{d-1}{2}-\frac{d}{p}-\varepsilon}\left(\mathbb{S}^{d}\right)
$$

où l'on note

$$
\forall s \in \mathbb{R} \quad H_{z o n}^{s}\left(\mathbb{S}^{d}\right):=\left\{\sum_{n \geq 1} a_{n} Z_{n}, \quad \sum_{n \geq 1} n^{2 s}\left|a_{n}\right|^{2}<+\infty\right\} \subset H^{s}\left(\mathbb{S}^{d}\right) .
$$

De même, le théorème 1.2 découle du résultat suivant.

Proposition 3.2. - On considère une suite complexe $\left(a_{n}\right)_{n \geq 1}$ à croissance polynomiale. Pour tout réel $p \in] 1,+\infty\left[\right.$, la distribution $\sum_{n \geq 1} a_{n} Y_{n}$ appartient à $\mathbf{P} \mathbf{L}^{p}\left(\mathbb{S}^{d}, \oplus \mathbb{C} Y_{n}\right)$ si et seulement si l'on a la condition

$$
\sum_{n \geq 1} \frac{1}{n^{\frac{d+1}{2}}}\left(\sum_{k=1}^{n} k^{\frac{d-1}{2}}\left|a_{k}\right|^{2}\right)^{\frac{p}{2}}<+\infty .
$$

En outre, les espaces $\mathbf{P} \mathbf{L}^{p}\left(\mathbb{S}^{d}, \oplus \mathbb{C} Y_{n}\right)$ sont stables par dualité et interpolation réelle et complexe pour $p$ parcourant $] 1,+\infty[$ au sens des théorèmes 2.5 et 2.6.

Par dualité, on va obtenir gratuitement la moitié des injections de Sobolev probabilistes des fonctions $Y_{n}$. Par commodité, on note

$$
\forall s \in \mathbb{R} \quad \widetilde{H}^{s}\left(\mathbb{S}^{d}\right):=\left\{\sum_{n \geq 0} a_{n} Y_{n}, \quad \sum_{n \geq 1} n^{2 s}\left|a_{n}\right|^{2}<+\infty\right\} \subset H^{s}\left(\mathbb{S}^{d}\right) .
$$

Corollaire 3.3. - Considérons $p \in] 2,+\infty\left[\right.$ et $\left.q=\frac{p}{p-1} \in\right] 1,2[$. Nous avons les inclusions

$$
\begin{aligned}
& \widetilde{H}^{\frac{d-1}{2}\left(\frac{1}{2}-\frac{1}{p}\right)}\left(\mathbb{S}^{d}\right) \subset \mathbf{P L}^{p}\left(\mathbb{S}^{d}, \oplus \mathbb{C} Y_{n}\right) \subset \bigcap_{\varepsilon>0} \widetilde{H}^{\frac{d-1}{2}\left(\frac{1}{2}-\frac{1}{p}\right)-\varepsilon}\left(\mathbb{S}^{d}\right), \\
& \bigcup_{\varepsilon>0} \widetilde{H}^{\frac{-(d-1)}{2}\left(\frac{1}{q}-\frac{1}{2}\right)+\varepsilon}\left(\mathbb{S}^{d}\right) \quad \subset \quad \mathbf{P L}^{q}\left(\mathbb{S}^{d}, \oplus \mathbb{C} Y_{n}\right) \subset \widetilde{H}^{\frac{\varepsilon>0}{2}}\left(\frac{1}{q}-\frac{1}{2}\right)\left(\mathbb{S}^{d}\right) \text {. }
\end{aligned}
$$

Pour conclure cette partie, remarquons que (82) et le corollaire 3.3 montrent l'optimalité de l'exposant $\delta(d, p)$ dans l'injection de Sobolev probabiliste de Tzvetkov (3). Comme expliqué dans l'introduction, cela est lié au fait que les fonctions $Y_{n}$ et $Z_{n}$ optimisent par leur concentration les normes dans $L^{p}\left(\mathbb{S}^{d}\right)$.

3.2. Preuve de la proposition 3.2, randomisation des fonctions $Y_{n}$. - Nous commençons par traiter les fonctions $Y_{n}$ car les idées d'interpolation sont plus simples. Rappelons en quel sens la fonction $Y_{n}$ se concentre de façon gaussienne sur une bande de largeur $\frac{1}{\sqrt{n}}$ autour de la géodésique $\left\{x_{1}^{2}+x_{2}^{2}=1\right\}$. On part des inégalités

$$
\forall \delta \in\left[0, \frac{\pi}{2}\right] \quad 1-\frac{\delta^{2}}{2} \leq \cos (\delta) \leq e^{-\frac{1}{2} \delta^{2}} .
$$

Le nombre $\delta=\arccos \left(\sqrt{x_{1}^{2}+x_{2}^{2}}\right)$ désigne la distance géodésique d'un point $x \in \mathbb{S}^{d}$ au cercle $\left\{x_{1}^{2}+x_{2}^{2}=\right.$ $1\} \subset \mathbb{S}^{d}$, puis la définition $Y_{n}(x)=c_{d, n}\left(x_{1}+i x_{2}\right)^{n}$ et l'équivalent $c_{d, n} \simeq_{d} n^{\frac{d-1}{4}}$ assurent qu'il existe $C(d)>1$ de sorte que

$$
\forall x \in \mathbb{S}^{d} \quad \forall n \in \mathbb{N}^{\star} \quad \frac{1}{C(d)} \mathbf{1}_{\left\{\arccos \left(\sqrt{x_{1}^{2}+x_{2}^{2}}\right) \leq \frac{1}{\sqrt{n}}\right\}} \leq \frac{\left|Y_{n}(x)\right|}{n^{\frac{d-1}{4}}} \leq C(d) e^{-\frac{1}{2} n\left[\arccos \left(\sqrt{x_{1}^{2}+x_{2}^{2}}\right)\right]^{2}} .
$$

L'idée que l'on doit garder à l'esprit est que l'on peut sous certaines conditions assimiler $\left|Y_{n}\right|$ à la fonction $\widetilde{Y}_{n}$ définie comme suit

$$
\widetilde{Y}_{n}(x):=n^{\frac{d-1}{4}} \mathbf{1}_{\left\{\arccos \left(\sqrt{x_{1}^{2}+x_{2}^{2}}\right) \leq \frac{1}{\sqrt{n}}\right\}} .
$$


Pour tout $p>1$, on peut alors vérifier les équivalences

$$
\left\|\widetilde{Y}_{n}\right\|_{L^{p}\left(\mathbb{S}^{d}\right)} \simeq_{d, p}\left\|Y_{n}\right\|_{L^{p}\left(\mathbb{S}^{d}\right)} \simeq_{d, p} n^{\frac{d-1}{2}\left(\frac{1}{2}-\frac{1}{p}\right)},
$$

ce qui signifie que la concentration autour de la géodésique $\left\{x_{1}^{2}+x_{2}^{2}=1\right\}$ est significative dans $L^{p}\left(\mathbb{S}^{d}\right)$.

Venons-en maintenant aux estimées multilinéaires des fonctions $Y_{n}$ qui font disparaître la plus grande fréquence. Le résultat suivant énonce que les estimées multilinéaires des fonctions $Y_{n}$ et $\widetilde{Y}_{n}$ sont équivalentes.

Lemme 3.4. - Pour tout entier $\alpha \geq 2$ et pour tous entiers naturels $n_{1} \geq \cdots \geq n_{\alpha} \geq 1$, on a

$$
\begin{gathered}
\int_{\mathbb{S}^{d}}\left|Y_{n_{1}}(x) \cdots Y_{n_{\alpha}}(x)\right|^{2} d \mu_{d}(x) \quad \simeq_{d, \alpha} \quad\left(n_{2} \cdots n_{\alpha}\right)^{\frac{d-1}{2}}, \\
\int_{\mathbb{S}^{d}}\left|\widetilde{Y}_{n_{1}}(x) \cdots \widetilde{Y}_{n_{\alpha}}(x)\right|^{2} d \mu_{d}(x) \simeq_{d, \alpha} \quad\left(n_{2} \cdots n_{\alpha}\right)^{\frac{d-1}{2}} .
\end{gathered}
$$

Preuve. On va invoquer l'argument algébrique de [8, pages 5-8], on a pour tout $x \in \mathbb{S}^{d}$ :

$$
Y_{n_{1}}(x) \ldots Y_{n_{\alpha}}(x)=c_{d, n_{1}} \ldots c_{d, n_{\alpha}}\left(x_{1}+i x_{2}\right)^{n_{1}+\cdots+n_{\alpha}}=\frac{c_{d, n_{1}} \ldots c_{d, n_{\alpha}}}{c_{d, n_{1}+\cdots+n_{\alpha}}} Y_{n_{1}+\cdots+n_{\alpha}}(x) .
$$

Et donc

$$
\int_{\mathbb{S}^{d}}\left|Y_{n_{1}}(x) \ldots Y_{n_{\alpha}}(x)\right|^{2} d \mu_{d}(x)=\left(\frac{c_{d, n_{1}} \ldots c_{d, n_{\alpha}}}{c_{d, n_{1}+\cdots+n_{\alpha}}}\right)^{2} \simeq_{d, \alpha}\left(\frac{n_{1} \ldots n_{\alpha}}{n_{1}+\cdots+n_{\alpha}}\right)^{\frac{d-1}{2}} .
$$

On conclut en invoquant les inégalités $n_{1} \leq n_{1}+\cdots+n_{\alpha} \leq \alpha n_{1}$.

Les intégrales multilinéaires des fonctions $\widetilde{Y}_{n}$ sont faciles à calculer l'aide d'une formule de changement de variables (voir plus loin (89)) en tenant compte que $n_{1}$ est le plus grand entier parmi $n_{2}, \ldots, n_{\alpha}$ :

$$
\begin{aligned}
\frac{\int_{\mathbb{S}^{d}}\left|\widetilde{Y}_{n_{1}}(x) \ldots \widetilde{Y}_{n_{\alpha}}(x)\right|^{2} d \mu_{d}(x)}{\left(n_{1} \cdots n_{\alpha}\right)^{\frac{d-1}{2}}} & =\int_{\mathbb{S}^{d}} \mathbf{1}_{\left\{\arccos \left(\sqrt{x_{1}^{2}+x_{2}^{2}}\right) \leq \frac{1}{\sqrt{n_{1}}}\right\}} d \mu_{d}(x) \\
& =\mu_{d-2}\left(\mathbb{S}^{d-2}\right) \int_{x_{1}^{2}+x_{2}^{2}<1}\left(1-x_{1}^{2}-x_{2}^{2}\right)^{\frac{d-3}{2}} \mathbf{1}_{\left\{\arccos \left(\sqrt{x_{1}^{2}+x_{2}^{2}}\right) \leq \frac{1}{\sqrt{n_{1}}}\right\}} d x_{1} d x_{2} \\
& =\mu_{d-2}\left(\mathbb{S}^{d-2}\right) \int_{\cos \left(\frac{1}{\sqrt{n_{1}}}\right)}^{1}\left(1-r^{2}\right)^{\frac{d-3}{2}} r d r \\
& =\mu_{d-2}\left(\mathbb{S}^{d-2}\right) \int_{0}^{\frac{1}{\sqrt{n_{1}}}} \sin ^{d-2}(u) \cos (u) d u \\
& \simeq \frac{1}{n_{1}^{\frac{d-1}{2}}} .
\end{aligned}
$$

Proposition 3.5. - Pour tout réel $p \in\left[1,+\infty\left[\right.\right.$ et pour toute suite complexe $\left(a_{n}\right)_{n \geq 1}$, on a

$$
\left\|\sqrt{\sum_{n \geq 1}\left|a_{n} \tilde{Y}_{n}\right|^{2}}\right\|_{L^{p}\left(\mathbb{S}^{d}\right)} \simeq_{d, p}\left[\sum_{n \geq 1} \frac{1}{n^{\frac{d+1}{2}}}\left(\sum_{k=1}^{n} k^{\frac{d-1}{2}}\left|a_{k}\right|^{2}\right)^{\frac{p}{2}}\right]^{\frac{1}{p}} .
$$

Preuve. Pour tout $x \in \mathbb{S}^{d}$, il s'agit de décomposer en supports disjoints

$$
\begin{aligned}
\sum_{n \geq 1}\left|a_{n} \widetilde{Y}_{n}(x)\right|^{2} & =\sum_{n \geq 1} n^{\frac{d-1}{2}}\left|a_{n}\right|^{2} \mathbf{1}_{\left\{\arccos \left(\sqrt{x_{1}^{2}+x_{2}^{2}}\right) \leq \frac{1}{\sqrt{n}}\right\}} \\
& =\sum_{n \geq 1}\left(\sum_{k=1}^{n} k^{\frac{d-1}{2}}\left|a_{k}\right|^{2}\right) \mathbf{1}_{\left\{\frac{1}{\sqrt{n+1}}<\arccos \left(\sqrt{x_{1}^{2}+x_{2}^{2}}\right) \leq \frac{1}{\sqrt{n}}\right\}} .
\end{aligned}
$$


En utilisant une expression de la forme $\sin ^{d-2}(\xi) \cos (\xi)=\xi^{d-2} G\left(\xi^{2}\right)$ avec $G$ fonction holomorphe, on peut assurer l'existence d'un réel $c \in \mathbb{R}$ qui précise la formule (86) :

$$
\int_{\mathbb{S}^{d}} \mathbf{1}_{\left\{\arccos \left(\sqrt{x_{1}^{2}+x_{2}^{2}}\right) \leq \frac{1}{\sqrt{n}}\right\}} d \mu_{d}(x)=\frac{\mu_{d-2}\left(\mathbb{S}^{d-2}\right)}{n^{\frac{d-1}{2}}}\left(1+\frac{c}{n}+\mathcal{O}\left(\frac{1}{n^{2}}\right)\right) .
$$

On conclut alors aisément.

Le résultat suivant dit précisément que l'approximation de $\left|Y_{n}\right|$ par $\widetilde{Y}_{n}$ est légitime dans la théorie $L^{p}$ probabiliste des modes propres $Y_{n}$.

Proposition 3.6. - Pour tout réel $p \in] 1,+\infty\left[\right.$ et pour toute suite complexe $\left(a_{n}\right)_{n \geq 1}$, on a

$$
\frac{1}{C(p, d)}\left\|\sqrt{\sum_{n \geq 1}\left|a_{n} \tilde{Y}_{n}\right|^{2}}\right\|_{L^{p}\left(\mathbb{S}^{d}\right)} \leq\left\|\sqrt{\sum_{n \geq 1}\left|a_{n} Y_{n}\right|^{2}}\right\|_{L^{p}\left(\mathbb{S}^{d}\right)} \leq C(p, d)\left\|\sqrt{\sum_{n \geq 1}\left|a_{n} \tilde{Y}_{n}\right|^{2}}\right\|_{L^{p}\left(\mathbb{S}^{d}\right)} .
$$

Preuve. Si $p=2 \alpha$ est un entier pair non nul, (87) découle du lemme 3.4 et des deux formules

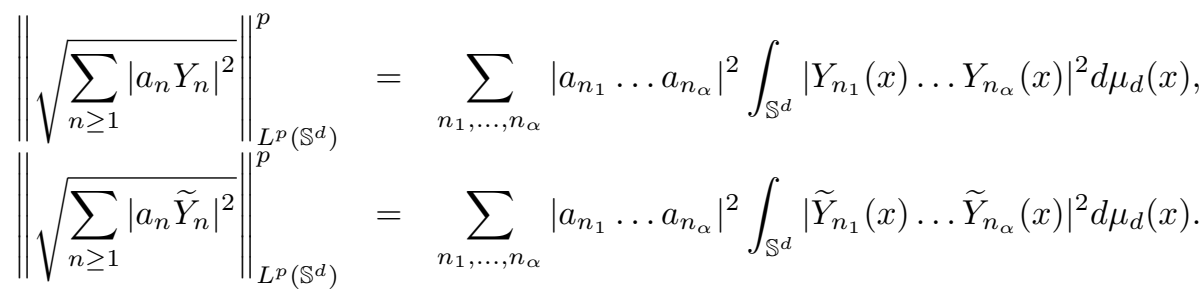

Pour assurer que l'équivalence (87) est encore valide pour tout $p \geq 2$, il nous suffit de prouver que les espaces $\mathbf{P L}^{p}\left(\mathbb{S}^{d}, \oplus \mathbb{C} Y_{n}\right)$ et $\mathbf{P} \mathbf{L}^{p}\left(\mathbb{S}^{d}, \oplus \mathbb{C} \widetilde{Y}_{n}\right)$ sont stables par interpolation au sens du théorème 2.5. Pour conclure, il suffira d'invoquer un argument de dualité (grâce au théorème 2.6). En effet, pour la dualité on aurait pour $q=\frac{p}{p-1}$ :

$$
\left\|\sqrt{\sum_{n \geq 1}\left|a_{n} \widetilde{Y}_{n}\right|^{2}}\right\|_{L^{q}(\mathbb{S} d)} \simeq \sup _{d, p} \frac{\left|\sum_{n \geq 1}\left\langle a_{n} \widetilde{Y}_{n}, b_{n} \widetilde{Y}_{n}\right\rangle\right|}{\left\|\sqrt{\sum_{n \geq 1}\left|b_{n} \tilde{Y}_{n}\right|^{2}}\right\|_{L^{p}\left(\mathbb{S}^{d}\right)}} .
$$

Quitte à remplacer $b_{n}$ par $\frac{b_{n}}{\left\|\widetilde{Y}_{n}\right\|_{L^{2}\left(\mathbb{S}^{d}\right)}^{2}}$ et en remarquant l'équivalence $\left\|\widetilde{Y}_{n}\right\|_{L^{2}\left(\mathbb{S}^{d}\right)} \simeq_{d} 1$ (voir (85)), l'expression précédente devient

$$
\begin{aligned}
\left\|\sqrt{\sum_{n \geq 1}\left|a_{n} \tilde{Y}_{n}\right|^{2}}\right\|_{L^{q}\left(\mathbb{S}^{d}\right)} & \simeq_{d, p} \sup _{\left(b_{n}\right)_{n \geq 1} \neq 0} \frac{\left|\sum_{n \geq 1} a_{n} b_{n}\right|}{\|\left.\sqrt{\sum_{n \geq 1}\left|b_{n} \widetilde{Y}_{n}\right|^{2}}\right|_{L^{p}\left(\mathbb{S}^{d}\right)}} \\
& \simeq_{d, p} \sup _{\left(b_{n}\right)_{n \geq 1} \neq 0} \frac{\left|\sum_{n \geq 1} a_{n} b_{n}\right|}{\left\|\sqrt{\sum_{n \geq 1}\left|b_{n} Y_{n}\right|^{2}}\right\|_{L^{p}\left(\mathbb{S}^{d}\right)}} \\
& \simeq_{d, p}\left\|\sqrt{\sum_{n \geq 1}\left|a_{n} Y_{n}\right|^{2}}\right\|_{L^{q}\left(\mathbb{S}^{d}\right)} .
\end{aligned}
$$

Validons maintenant les hypothèses des théorèmes d'interpolation et dualité. En examinant (85) et en se rappelant que $\frac{1}{2}-\frac{1}{p}$ change de signe en remplaçant $p$ par son exposant conjugué $\frac{p}{p-1}$, on comprend que l'hypothèse (37) est vérifiée avec $\sqrt{e_{n}(x)}=\left|Y_{n}(x)\right|$ et $\sqrt{e_{n}(x)}=\left\|\widetilde{Y}_{n}\right\|_{L^{2}\left(\mathbb{S}^{d}\right)}^{-1} \tilde{Y}_{n}(x)$.

Quant à l'hypothèse (35), elle va s'avérer être une conséquence de la concentration gaussienne des fonctions $Y_{n}$. En effet, grâce à (84) et (85), nous avons pour tout $n \in \mathbb{N}^{\star}$ et $x \in \mathbb{S}^{d}$

$$
\frac{\left|Y_{n}(x)\right|}{\left\|Y_{n}\right\|_{L^{p}\left(\mathbb{S}^{d}\right)}} \leq C(d, p) n^{\frac{d-1}{2 p}} e^{-\frac{n}{2}\left[\arccos \left(\sqrt{x_{1}^{2}+x_{2}^{2}}\right)\right]^{2}} \leq \frac{C(d, p)}{\arccos \left(\sqrt{x_{1}^{2}+x_{2}^{2}}\right)^{\frac{d-1}{p}}} .
$$


Et il se trouve que $x \mapsto \arccos \left(\sqrt{x_{1}^{2}+x_{2}^{2}}\right)^{-\frac{(d-1)}{p}}$ appartient à l'espace de Lorentz $L^{p, \infty}\left(\mathbb{S}^{d}\right)$, ou ce qui revient au même que $x \mapsto \arccos \left(\sqrt{x_{1}^{2}+x_{2}^{2}}\right)^{-(d-1)}$ appartient à $L^{1, \infty}\left(\mathbb{S}^{d}\right)$ : la formule de changement de variables (89) donne pour tout $t>1$

$$
\begin{aligned}
\mu_{d}\left\{x \in \mathbb{S}^{d},\right. & \left.\arccos \left(\sqrt{x_{1}^{2}+x_{2}^{2}}\right)^{-(d-1)}>t\right\} \\
& \simeq_{d} \int_{x_{1}^{2}+x_{2}^{2}<1}\left(1-x_{1}^{2}-x_{2}^{2}\right)^{\frac{d-3}{2}} \mathbf{1}_{\left\{\arccos \left(\sqrt{x_{1}^{2}+x_{2}^{2}}\right)^{-(d-1)}>t\right\}} d x_{1} d x_{2} \\
& \simeq_{d} \int_{\cos \left(t^{\frac{-1}{d-1}}\right)}^{1}\left(1-r^{2}\right)^{\frac{d-3}{2}} r d r \\
& \simeq_{d} \int_{\cos \left(t^{\frac{-1}{d-1}}\right)}^{1}(1-r)^{\frac{d-3}{2}} d r \\
& \simeq_{d}\left[1-\cos \left(t^{\frac{-1}{d-1}}\right)\right]^{\frac{d-1}{2}} \\
& \lesssim_{d} \frac{1}{t}
\end{aligned}
$$

Comme $\mu_{d}\left(\mathbb{S}^{d}\right)$ est fini, l'estimation précédente est aussi valide si $t$ appartient à ]0,1].

Remarque 3.7. - Concernant l'hypothèse (35), l'intérêt des espaces de Lorentz est désormais flagrant. En effet, en utilisant la minoration de (84), nous arrivons à

$$
\begin{aligned}
\forall x \in \mathbb{S}^{d} \sup _{n \geq 1} \frac{\left|Y_{n}(x)\right|^{p}}{\left\|Y_{n}\right\|_{L^{p}\left(\mathbb{S}^{d}\right)}^{p}} & \geq C(d, p) \sup _{n \geq 1} n^{\frac{d-1}{2}} \mathbf{1}_{\left\{\arccos \left(\sqrt{x_{1}^{2}+x_{2}^{2}}\right) \leq \frac{1}{\sqrt{n}}\right\}} \\
& \geq C(d, p) \arccos \left(\sqrt{x_{1}^{2}+x_{2}^{2}}\right)^{-(d-1)} .
\end{aligned}
$$

Or (88) est une équivalence si t tend vers $+\infty$. Cela implique que $x \mapsto \arccos \left(\sqrt{x_{1}^{2}+x_{2}^{2}}\right)^{-(d-1)}$ n'est pas intégrable sur $\mathbb{S}^{d}$. Il s'ensuit que $\sup _{n \geq 1} \frac{\left|Y_{n}\right|}{\left\|Y_{n}\right\|_{L^{p}\left(\mathbb{S}^{d}\right)}}$ n'appartient pas à $L^{p}\left(\mathbb{S}^{d}\right)$.

La proposition 3.2 est alors une conséquence des propositions 3.5 et 3.6 et des théorèmes 2.5 et 2.6. On conclut avec une formule standard de changement de variables.

Proposition 3.8. - Considérons un entier naturel $k \in[1, d]$ et une fonction intégrable $f: \mathbb{S}^{d} \rightarrow \mathbb{C}^{q u i}$ ne dépend que des $k$ premières coordonnées $y=\left(x_{1}, x_{2}, \ldots, x_{k}\right)$. En notant $\widetilde{f}(y)$ la valeur commune des nombres $f(y, z)$ pour $(y, z) \in \mathbb{S}^{d}$, on a

$$
\int_{\mathbb{S}^{d}} f(x) d \mu_{d}(x)=\mu_{d-k}\left(\mathbb{S}^{d-k}\right) \int_{\mathbb{B}_{k}(0,1)} \tilde{f}(y)\left(1-|y|^{2}\right)^{\frac{d-k-1}{2}} d y,
$$

où l'on a noté $\mathbb{B}_{k}(0,1):=\left\{y \in \mathbb{R}^{k},|y|<1\right\}$. Dans le cas $k=d$, on convient que $\mu_{0}\left(\mathbb{S}^{0}\right)=2$.

Preuve. On donne les principales lignes la preuve du cas $k \leq d-1$. Le cas $k=d$ se traite de même. On introduit le changement de variables

$$
\begin{aligned}
\Psi: \mathbb{B}_{k}(0,1) \backslash\{0\} \times \mathbb{S}^{d-k} & \rightarrow \mathbb{S}^{d} \\
(y, u) & \mapsto\left(y,\left(1-|y|^{2}\right)^{\frac{1}{2}} u\right)
\end{aligned}
$$


dont la différentielle au point $(y, u)$ est l'application linéaire

$$
\begin{aligned}
D_{(y, u)} \Psi: \mathbb{R}^{k} \times T_{u} \mathbb{S}^{d-k} & \rightarrow T_{\Psi(y, u)} \mathbb{S}^{d} \\
(\eta, w) & \mapsto\left(\eta, \frac{-\langle y, \eta\rangle}{\sqrt{1-|y|^{2}}} u+\left(1-|y|^{2}\right)^{\frac{1}{2}} w\right) .
\end{aligned}
$$

L'injectivité de $\Psi$ et l'inversibilité de $D_{(y, u)} \Psi$ pour tout $(y, u)$ sont immédiates de sorte que le théorème d'inversion globale sur les variétés montre que $\Psi$ est $\mathcal{C}^{1}$-difféomorphisme sur son image (qui est de mesure pleine dans $\mathbb{S}^{d}$ ). Il nous reste à étudier le transport des formes volumes. Considérons d'abord dans $\mathbb{R}^{k}$ une base orthonormée de la forme $\frac{y}{|y|}, \overrightarrow{\xi_{2}}, \ldots, \overrightarrow{\xi_{k}}$, puis dans $T_{u} \mathbb{S}^{d-k}$ une base orthonormée quelconque $\overrightarrow{\vartheta_{1}}, \ldots, \overrightarrow{\vartheta_{d-k}}$. Ainsi,

$$
\left(\frac{y}{|y|}, 0\right),\left(\overrightarrow{\xi_{2}}, 0\right), \ldots,\left(\overrightarrow{\xi_{k}}, 0\right),\left(0, \overrightarrow{\vartheta_{1}}\right), \ldots,\left(0, \overrightarrow{\vartheta_{d-k}}\right)
$$

est une base orthonormée de $\mathbb{R}^{k} \times T_{u} \mathbb{S}^{d-k}$. Il s'avère que cette base orthonormée est envoyée sur une famille orthogonale de l'espace tangent $T_{\Psi(y, u)} \mathbb{S}^{d}$ :

$$
\begin{aligned}
& D_{(y, u)} \Psi\left(\frac{y}{|y|}, 0\right)=\frac{1}{\sqrt{1-|y|^{2}}}\left(\frac{y}{|y|} \sqrt{1-|y|^{2}},-|y| u\right) \\
& 2 \leq i \leq k, \quad D_{(y, u)} \Psi\left(\overrightarrow{\xi_{i}}, 0\right)=\left(\overrightarrow{\xi_{i}}, 0\right) \\
& 1 \leq j \leq d-k, \quad D_{(y, u)} \Psi\left(0, \overrightarrow{\vartheta_{j}}\right)=\sqrt{1-|y|^{2}}\left(0, \overrightarrow{\vartheta_{j}}\right) .
\end{aligned}
$$

Par conséquent, $D_{(y, u)} \Psi$ multiplie localement les volumes par $\left(1-|y|^{2}\right)^{\frac{d-k-1}{2}}$. Le changement de variables $x=\Psi(y, u)$ nous amène à la formule

$$
\begin{aligned}
\int_{\mathbb{S}^{d}} f(x) d \mu_{d}(x) & =\int_{\mathbb{B}_{k}(0,1) \times \mathbb{S}^{d-k}} f\left(y, u \sqrt{1-|y|^{2}}\right)\left(1-|y|^{2}\right)^{\frac{d-k-1}{2}} d y d \mu_{d-k}(u) \\
& =\mu_{d-k}\left(\mathbb{S}^{d-k}\right) \int_{\mathbb{B}_{k}(0,1)} \widetilde{f}(y)\left(1-|y|^{2}\right)^{\frac{d-k-1}{2}} d y .
\end{aligned}
$$

3.3. Preuve du corollaire 3.3, injections de Sobolev probabilistes des fonctions $Y_{n}$. - Nous avons vu dans la preuve de la proposition 3.6 que les espaces $\mathbf{P L}^{p}\left(\mathbb{S}^{d}, \oplus \mathbb{C} Y_{n}\right)$ sont stables par dualité. Comme il en est de même des espaces de Sobolev, on peut se restreindre au cas $p>2$.

L'inégalité triangulaire (2) et les estimations (10) donnent immédiatement l'inclusion $\widetilde{H}^{\frac{d-1}{2}\left(\frac{1}{2}-\frac{1}{p}\right)}\left(\mathbb{S}^{d}\right) \subset$ $\mathbf{P L}^{p}\left(\mathbb{S}^{d}, \oplus \mathbb{C} Y_{n}\right)$.

Montrons maintenant l'inclusion $\mathbf{P L}^{p}\left(\mathbb{S}^{d}, \oplus \mathbb{C} Y_{n}\right) \subset \bigcap_{\varepsilon>0} \widetilde{H}^{\frac{d-1}{2}\left(\frac{1}{2}-\frac{1}{p}\right)-\varepsilon}\left(\mathbb{S}^{d}\right)$. Autrement dit, pour toute distribution $u=\sum_{n \geq 1} a_{n} Y_{n}$, il s'agit de vérifier l'inégalité suivante

$$
\|u\|_{H^{\frac{d-1}{2}\left(\frac{1}{2}-\frac{1}{p}\right)-\frac{\varepsilon}{2}\left(\mathbb{S}^{d}\right)}} \lesssim_{d, p, \varepsilon}\|u\|_{\mathbf{P L}^{p}\left(\mathbb{S}^{d}, \oplus \mathbb{C} Y_{n}\right)} .
$$


On pose $S_{0}=0$ et $S_{n}=\sum_{k=1}^{n} k^{\frac{d-1}{2}}\left|a_{k}\right|^{2}$ pour tout $n \geq 1$. En utilisant une transformation d'Abel et une inégalité de Hölder, on a

$$
\begin{aligned}
& \|u\|_{H^{\frac{d-1}{2}\left(\frac{1}{2}-\frac{1}{p}\right)-\frac{\varepsilon}{2}}\left(\mathbb{S}^{d}\right)}^{2}=\sum_{n>1} n^{(d-1)\left(\frac{1}{2}-\frac{1}{p}\right)-\varepsilon}\left|a_{n}\right|^{2} \\
& =\sum_{n \geq 1}^{n \geq 1} n^{(d-1)\left(\frac{1}{2}-\frac{1}{p}\right)-\varepsilon-\left(\frac{d-1}{2}\right)}\left[S_{n}-S_{n-1}\right] \\
& =\sum_{n \geq 1}^{n \geq 1} n^{-\frac{(d-1)}{p}-\varepsilon}\left[S_{n}-S_{n-1}\right] \\
& \lesssim_{d, p, \varepsilon} \lim _{N \rightarrow+\infty} \sum_{n=1}^{N} \frac{S_{n}}{n^{\frac{(d-1)}{p}+1+\varepsilon}}+\sup _{N \geq 1} \frac{S_{N}}{N^{\frac{(d-1)}{p}+\varepsilon}} \\
& \lesssim d, p, \varepsilon \quad \lim _{N \rightarrow+\infty} \sum_{n=1}^{N} \frac{S_{n}}{n^{\frac{(d-1)}{p}+1+\varepsilon}}+\sup _{N \geq 1} \sum_{n>N} \frac{S_{n}}{n^{\frac{(d-1)}{p}+1+\varepsilon}} \\
& \lesssim d, p, \varepsilon \quad \sum_{n \geq 1} \frac{S_{n}}{n^{\frac{(d-1)}{p}+1+\varepsilon}} \\
& \lesssim d, p, \varepsilon \quad \sum_{n \geq 1} \frac{1}{n^{1-\frac{2}{p}+\varepsilon}} \times \frac{S_{n}}{n^{\frac{d+1}{p}}} \\
& \lesssim_{d, p, \varepsilon}\left(\sum_{n \geq 1} \frac{S_{n}^{\frac{p}{2}}}{n^{\frac{d+1}{2}}}\right)^{\frac{2}{p}} \text {. }
\end{aligned}
$$

3.4. Normes $L^{p}$ des fonctions zonales. - Parmi les modes propres de $\Delta$, les fonctions $Z_{n}$ sont connues pour maximiser la croissance des quotients $\frac{\left\|Z_{n}\right\|_{L^{p}\left(\mathbb{S}^{d}\right)}}{\left\|Z_{n}\right\|_{L^{2}\left(\mathbb{S}^{d}\right)}}$ si $n$ tend vers $+\infty$ avec $p \geq \frac{2(d+1)}{d-1}$ (et cela est même optimal d'après les inégalités de Sogge (4)). En l'occurrence, la formule de changement de variables (89) donne

$$
\left\|Z_{n}\right\|_{L^{p}\left(\mathbb{S}^{d}\right)}^{p}=\int_{\mathbb{S}^{d}}\left|Z_{n}(x)\right|^{p} d \mu_{d}(x)=\mu_{d-1}\left(\mathbb{S}^{d-1}\right) \times n^{\frac{p}{2}} \int_{-1}^{1}\left|P_{n}^{\left(\frac{d-2}{2}, \frac{d-2}{2}\right)}\left(x_{1}\right)\right|^{p}\left(1-x_{1}^{2}\right)^{\frac{d-2}{2}} d x_{1},
$$

puis [54, Page 391] nous fournit les estimations des normes (6).

3.5. Preuve de la proposition 3.1, randomisation des fonctions zonales $Z_{n}$. — La description des espaces $\mathbf{P} \mathbf{L}^{p}\left(\mathbb{S}^{d}, \oplus \mathbb{C} Z_{n}\right)$ est plus délicate que celle des espaces $\mathbf{P} \mathbf{L}^{p}\left(\mathbb{S}^{d}, \oplus \mathbb{C} Y_{n}\right)$ car on ne peut pas raisonner par interpolation complexe en faisant parcourir $p$ dans $2 \mathbb{N}$. Cela dit, une fois cette description obtenue, les injections de Sobolev probabilistes (82) des fonctions $Z_{n}$ se démontrent de façon rigoureusement semblable à celle des fonctions $Y_{n}$ du corollaire 3.3 et l'on se permet d'en omettre la preuve. Avant d'expliquer plus en détail la difficulté rencontrée dans cette preuve par rapport à celle de la proposition 3.2, commençons par rappeler les estimations précises des polynômes de Jacobi.

Lemme 3.9. - Pour tout $\alpha>-1$, il existe des constantes $\left.c=c(\alpha) \in] 0, \frac{\pi}{2}\right]$ et $C(\alpha) \geq 1$ de sorte que pour tous $n \in \mathbb{N}^{\star}$ l'on a

$$
\Theta \in\left[0, \frac{c}{n}\right] \cup\left[\pi-\frac{c}{n}, \pi\right] \Rightarrow \frac{n^{\alpha}}{C(\alpha)} \leq\left|P_{n}^{(\alpha, \alpha)}(\cos (\Theta))\right| \leq C(\alpha) n^{\alpha} .
$$

On note ensuite $N=n+\alpha+\frac{1}{2}$ et $\varrho=\frac{\pi}{2}\left(\alpha+\frac{1}{2}\right)$. Si $\Theta$ appartient $\grave{a}\left[\frac{c}{n}, \pi-\frac{c}{n}\right]$ alors

$$
P_{n}^{(\alpha, \alpha)}(\cos (\Theta))=\frac{2^{\alpha+\frac{1}{2}}}{\sqrt{\pi n}(\sin \Theta)^{\alpha+\frac{1}{2}}}\left[\cos (N \Theta-\varrho)+\frac{\mathcal{O}_{\alpha}(1)}{n \sin (\Theta)}\right]
$$

où le terme $\mathcal{O}_{\alpha}(1)$ vérifie $\left|\mathcal{O}_{\alpha}(1)\right| \leq C(\alpha)$.

Preuve. Ces estimées découlent des formules (4.1.3),(4.21.7), (7.32.5) et (8.21.18) du livre [54]. La formule (4.1.3) nous donne $P_{n}^{(\alpha, \alpha)}\left(-x_{1}\right)=(-1)^{n} P_{n}^{(\alpha, \alpha)}\left(x_{1}\right)$, ce qui nous ramène au cas $\Theta \in\left[0, \frac{\pi}{2}\right]$. D'une part, on a toujours

$$
P_{n}^{(\alpha, \alpha)}(1)=\left(\begin{array}{c}
n+\alpha \\
n
\end{array}\right) \geq \frac{n^{\alpha}}{C(\alpha)} .
$$


Pour tout $x_{1} \in\left[1-\frac{1}{n^{2}}, 1\right]$, on peut estimer grâce aux formules (4.21.7) page 63 et (7.32.5) page 169 :

$$
\left|\frac{d}{d x_{1}} P_{n}^{(\alpha, \alpha)}\left(x_{1}\right)\right|=\frac{1}{2}\left|(n+2 \alpha+1) P_{n-1}^{(\alpha+1, \alpha+1)}\left(x_{1}\right)\right| \leq C(\alpha) n^{\alpha+2} .
$$

Choisissons $c(\alpha)=\frac{1}{2 C(\alpha)^{2}}$ de sorte que $\frac{1}{C(\alpha)}-c(\alpha) C(\alpha)=\frac{1}{2 C(\alpha)}$. On a

$$
1-\frac{c(\alpha)}{n^{2}} \leq x_{1} \leq 1 \quad \Rightarrow \quad P_{n}^{(\alpha, \alpha)}\left(x_{1}\right) \geq \frac{n^{\alpha}}{2 C(\alpha)} .
$$

De nouveau, d'après la formule (7.32.5) de la page 169 et quitte à augmenter $C(\alpha)>1$, on a aussi $P_{n}^{(\alpha, \alpha)}\left(x_{1}\right) \leq C(\alpha) n^{\alpha}$. Cela nous donne (90). Quant à (91), c'est la formule (8.21.18) de la page 198 .

Dans la suite, on notera

$$
\Theta:=\arccos \left(x_{1}\right) \in[0, \pi]
$$

la distance géodésique d'un point $x \in \mathbb{S}^{d}$ au pôle $(1,0, \ldots, 0)$. D'après (5), (90), (91) avec $\alpha=\frac{d-2}{2}$, on a

$$
\begin{gathered}
\Theta \in\left[0, \frac{c}{n}\right] \cup\left[\pi-\frac{c}{n}, \pi\right] \Rightarrow \frac{n^{\frac{d-1}{2}}}{C(d)} \leq\left|Z_{n}(x)\right| \leq C(d) n^{\frac{d-1}{2}}, \\
\Theta \in] \frac{c}{n}, \pi-\frac{c}{n}\left[\Rightarrow\left|Z_{n}(x)\right| \leq \frac{C(d)}{\sin (\Theta)^{\frac{d-1}{2}}} .\right.
\end{gathered}
$$

C'est d'ailleurs avec ces estimations que l'on peut obtenir les estimations (6) des normes dans $L^{p}\left(\mathbb{S}^{d}\right)$ des fonctions $Z_{n}$. Ces dernières disent que seule la concentration au voisinage des pôles est significative dans l'échelle des espaces $L^{p}\left(\mathbb{S}^{d}\right)$, avec $p>\frac{2 d}{d-1}$. Il est donc naturel de comparer $Z_{n}$ à sa restriction $\widetilde{Z}_{n}$ au voisinage du pôle $(1,0, \ldots, 0)$ :

$$
\widetilde{Z}_{n}(x):=\mathbf{1}_{\left[0, \frac{c}{n}\right]}(\Theta) \times Z_{n}(x) .
$$

La fonction $\widetilde{Z}_{n}$ se concentre sur une boule de centre $(1,0, \ldots, 0)$ de rayon $\frac{c}{n}$ et avec une amplitude d'ordre $n^{\frac{d-1}{2}}$. La preuve de la proposition 3.2 consistait à comparer $\left|Y_{n}\right|$ à sa restriction $\widetilde{Y}_{n}$ autour d'une géodésique. Le lemme 3.4 assurait alors que les fonctions $\left|Y_{n}\right|$ ont les mêmes estimations multilinéaires que les fonctions $\widetilde{Y}_{n}$. Malheureusement, il est illusoire de refaire le même argument en approchant les fonctions $Z_{n}$ par les fonctions $\widetilde{Z}_{n}$ par exemple pour étudier l'espace $\mathbf{P L}^{6}\left(\mathbb{S}^{d}, \oplus \mathbb{C} Z_{n}\right)$. Utilisant l'équivalent $\left\|Z_{n}\right\|_{L^{2}\left(\mathbb{S}^{d}\right)} \simeq_{d} 1,(92)$ et $[\mathbf{8}$, page 8$]$, nous avons en effet pour tout entier $n \in \mathbb{N}^{\star}$

$$
\int_{\mathbb{S}^{d}}\left|\widetilde{Z}_{1}(x)\right|^{4}\left|\widetilde{Z}_{n}(x)\right|^{2} d \mu_{d}(x) \leq C(d) \int_{\mathbb{S}^{d}}\left|\widetilde{Z}_{n}(x)\right|^{2} d \mu_{d}(x) \leq \frac{C(d)}{n} \ll \int_{\mathbb{S}^{d}}\left|Z_{1}(x)\right|^{4}\left|Z_{n}(x)\right|^{2} d \mu_{d}(x) .
$$

L'estimation précédente est une manifestation de la mauvaise qualité de l'approximation de $Z_{n}$ par $\widetilde{Z}_{n}$ dans $L^{2}\left(\mathbb{S}^{d}\right)$, phénomène qui ne se produit pas pour les fonctions $Y_{n}$. On ne dispose donc pas d'un résultat analogue au lemme 3.4. Cela nous oblige à raisonner différemment.

Proposition 3.10. - Pour tout réel $p>\frac{2 d}{d-1}$, il existe une constante $C(p, d) \geq 1$ telle que pour toute suite complexe $\left(a_{n}\right)_{n \geq 1}$ et tout $x \in \mathbb{S}^{d}$ vérifiant $\Theta(x) \in\left[0, \frac{\pi}{2}\right]$, on a

$$
\sqrt{\sum_{n \geq 1}\left|a_{n} Z_{n}(x)\right|^{2}} \leq C(p, d) \sqrt{\sum_{n \geq 1}\left|a_{n} \widetilde{Z}_{n}(x)\right|^{2}}+\frac{C(p, d)}{\sin ^{\frac{d}{p}}(\Theta)}\left\|\sqrt{\sum_{n \geq 1}\left|a_{n} \widetilde{Z}_{n}\right|^{2}}\right\|_{L^{p}\left(\mathbb{S}^{d}\right)} .
$$

Par conséquent, on a

$$
\left\|\sqrt{\sum_{n \geq 1}\left|a_{n} Z_{n}\right|^{2}}\right\|_{L^{p, \infty}\left(\mathbb{S}^{d}\right)} \leq C(p, d)\left\|\sqrt{\sum_{n \geq 1}\left|a_{n} \widetilde{Z}_{n}\right|^{2}}\right\|_{L^{p}\left(\mathbb{S}^{d}\right)} .
$$


Preuve. Sans perte de généralité, on suppose que la suite $\left(a_{n}\right)$ n'a qu'un nombre fini de termes non nuls. L'idée consiste essentiellement à décomposer les différentes fonctions en jeu en somme de fonctions à supports disjoints deux à deux. La formule de changement de variables (89) donne

$$
\begin{aligned}
\int_{\mathbb{S}^{d}} \mathbf{1}_{] \frac{c}{n+1}, \frac{c}{n}\right]}(\Theta) d \mu_{d}(x) & =\mu_{d-1}\left(\mathbb{S}^{d-1}\right) \int_{-1}^{1} \mathbf{1}_{] \frac{c}{n+1}, \frac{c}{n}\right]}(\Theta)\left(1-x_{1}^{2}\right)^{\frac{d-2}{2}} d x_{1} \\
& =\mu_{d-1}\left(\mathbb{S}^{d-1}\right) \int_{0}^{\pi} \mathbf{1}_{\left.\frac{c}{n+1}, \frac{c}{n}\right]}(\Theta) \sin (\Theta)^{d-1} d \Theta \\
& \simeq{ }_{d} \frac{1}{n^{d+1}} .
\end{aligned}
$$

En posant $S_{n}=\sum_{k=1}^{n} k^{d-1}\left|a_{k}\right|^{2}$ pour tout $n \in \mathbb{N}^{\star}$, nous avons grâce à (92) :

$$
\begin{aligned}
\sqrt{\sum_{n \geq 1}\left|a_{n} \widetilde{Z}_{n}(x)\right|^{2}} & \simeq d \sqrt{\sum_{n \geq 1} n^{d-1}\left|a_{n}\right|^{2} \mathbf{1}_{\left[0, \frac{c}{n}\right]}(\Theta)} \\
& \simeq \sum_{n \geq 1} \sqrt{S_{n}} \mathbf{1}_{\left.\frac{c}{n+1}, \frac{c}{n}\right]}(\Theta)
\end{aligned}
$$

et donc

$$
\begin{aligned}
\left\|\sqrt{\sum_{n \geq 1}\left|a_{n} \widetilde{Z}_{n}\right|^{2}}\right\|_{L^{p}\left(\mathbb{S}^{d}\right)} & \simeq_{d, p}\left(\sum_{n \geq 1} S_{n}^{\frac{p}{2}} \int_{\mathbb{S}^{d}} \mathbf{1}_{] \frac{c}{n+1}, \frac{c}{n}\right]}(\Theta) d \mu_{d}(x)\right)^{\frac{1}{p}} \\
& \simeq_{d, p}\left(\sum_{n \geq 1} \frac{S_{n}^{\frac{p}{2}}}{n^{d+1}}\right)^{\frac{1}{p}}
\end{aligned}
$$

On peut maintenant estimer $Z_{n}(x)-\widetilde{Z}_{n}(x)$. En tenant compte de $(93)$ et du fait que $\Theta(x)$ appartient à $\left[0, \frac{\pi}{2}\right]$, nous avons

$$
\begin{aligned}
\sqrt{\sum_{n \geq 1}\left|a_{n}\right|^{2}\left|Z_{n}(x)-\widetilde{Z}_{n}(x)\right|^{2}} & \lesssim_{d}\left(\sum_{k \geq 1}\left|a_{k}\right|^{2}\right)^{\frac{1}{2}} \frac{\mathbf{1}_{] c, \frac{\pi}{2}\right]}(\Theta)}{\sin (\Theta)^{\frac{d-1}{2}}}+\sum_{n \geq 1}\left(\sum_{k>n}\left|a_{k}\right|^{2}\right)^{\frac{1}{2}} \frac{\mathbf{1}_{] \frac{c}{n+1}, \frac{c}{n}\right]}(\Theta)}{\sin (\Theta)^{\frac{d-1}{2}}} \\
& \underbrace{\left(\sum_{k \geq 1}\left|a_{k}\right|^{2}\right)^{\frac{1}{2}} \mathbf{1}_{] c, \frac{\pi}{2}\right]}(\Theta)}_{:=A_{1}(\Theta)}+\underbrace{\sum_{n \geq 1}\left(n^{d-1} \sum_{k>n}\left|a_{k}\right|^{2}\right)^{\frac{1}{2}} \mathbf{1}_{] \frac{c}{n+1}, \frac{c}{n}\right]}(\Theta)}_{:=A_{2}(\Theta)} .
\end{aligned}
$$

On va faire quelques calculs avant d'attaquer l'estimation des termes $A_{1}(\Theta)$ et $A_{2}(\Theta)$. En convenant que $S_{0}=0$, nous pouvons effectuer une transformation d'Abel pour tout $n \in \mathbb{N}$

$$
\sum_{k>n}\left|a_{k}\right|^{2}=\sum_{k>n} \frac{k^{d-1}\left|a_{k}\right|^{2}}{k^{d-1}}=\frac{-S_{n}}{(n+1)^{d-1}}+\sum_{k>n}\left(\frac{1}{k^{d-1}}-\frac{1}{(k+1)^{d-1}}\right) S_{k} .
$$

Puisque la suite $\left(a_{n}\right)_{n \geq 1}$ n'a qu'un nombre fini de termes non nuls, la suite $\left(S_{n}\right)_{n \geq 0}$ est bornée et la dernière série converge bien. On va exploiter l'inégalité $p>\frac{2 d}{d-1}$, ou encore

$$
\left(d-\frac{2(d+1)}{p}\right) \frac{p}{p-2}=d-\frac{2}{p-2}>1 \text {. }
$$


L'inégalité de Hölder avec les exposants conjugués $\frac{p}{p-2}, \frac{p}{2}$ :

$$
\begin{aligned}
(n+1)^{d-1} \sum_{k>n} \frac{S_{k}}{k^{d}} & \leq \quad(n+1)^{d-1} \sum_{k>n} \overbrace{\frac{1}{k^{d-\frac{2(d+1)}{p}}}}^{\epsilon \ell^{\frac{p}{p-2}}(\mathbb{N})} \times \frac{S_{k}}{k^{\frac{2(d+1)}{p}}} \\
& \leq \quad(n+1)^{d-1}\left(\sum_{k>n} \frac{1}{k^{d-\frac{2}{p-2}}}\right)^{\frac{p-2}{p}}\left(\sum_{k>n} \frac{S_{k}^{\frac{p}{2}}}{k^{d+1}}\right)^{\frac{2}{p}} \\
& \lesssim_{d, p} \frac{(n+1)^{d-1}}{(n+1)^{\frac{p-2}{p}\left(d-\frac{2}{p-2}-1\right)}}\left(\sum_{k>n} \frac{S_{k}^{\frac{p}{2}}}{k^{d+1}}\right)^{\frac{2}{p}} \\
& \lesssim_{d, p}(n+1)^{\frac{2 d}{p}}\left(\sum_{k>n} \frac{S_{k}^{\frac{p}{2}}}{k^{d+1}}\right)^{\frac{2}{p}} \\
& \lesssim_{d, p} \quad(n+1)^{\frac{2 d}{p}}\left\|\sqrt{\sum_{n \geq 1}\left|a_{n} \widetilde{Z}_{n}\right|^{2}}\right\|_{L^{p}\left(\mathbb{S}^{d}\right)}^{2}
\end{aligned}
$$

où l'on a utilisé (97). On peut alors contrôler $A_{1}(\Theta)$ avec (98) et (100) pour $n=0$ :

$$
A_{1}(\Theta)=\sqrt{\sum_{k \geq 1}\left|a_{k}\right|^{2}} \times \mathbf{1}_{] c, \frac{\pi}{2}[}(\Theta) \lesssim_{d, p}\left\|\sqrt{\sum_{n \geq 1}\left|a_{n} \widetilde{Z}_{n}\right|^{2}}\right\|_{L^{p}\left(\mathbb{S}^{d}\right)} \frac{1}{\sin ^{\frac{d}{p}}(\Theta)} .
$$

Pour contrôler $A_{2}(\Theta)$, on utilise (96), (98) et (100) :

$$
\begin{aligned}
A_{2}(\Theta) & \lesssim_{d, p} \quad \sum_{n \geq 1} \sqrt{S_{n}} \mathbf{1}_{\left.\frac{c}{n+1}, \frac{c}{n}\right]}(\Theta)+\sum_{n \geq 1} n^{\frac{d}{p}}\left\|\sqrt{\sum_{n \geq 1}\left|a_{n} \widetilde{Z}_{n}\right|^{2}}\right\|_{L^{p}\left(\mathbb{S}^{d}\right)} \mathbf{1}_{\left.\frac{c}{n+1}, \frac{c}{n}\right]}(\Theta) \\
& \lesssim_{d, p} \sqrt{\sum_{n \geq 1}\left|a_{n} \widetilde{Z}_{n}(x)\right|^{2}}+\left\|\sqrt{\sum_{n \geq 1}\left|a_{n} \widetilde{Z}_{n}\right|^{2}}\right\|_{L^{p}\left(\mathbb{S}^{d}\right)} \frac{1}{\sin ^{\frac{d}{p}}(\Theta)} .
\end{aligned}
$$

On a donc obtenu (94). Passons à l'inégalité (95). Comme les polynômes de Jacobi sont pairs ou impairs, les fonctions $x \mapsto\left|Z_{n}(x)\right|$ sont invariantes par la transformation $\Theta(x) \rightarrow \pi-\Theta(x)$. Se rappelant que $\|\cdot\|_{L^{p, \infty}\left(\mathbb{S}^{d}\right)}$ n'est pas une norme, on sait qu'il existe néanmoins une constante universelle $C \geq 1$ telle que

$$
\left\|\sqrt{\sum_{n \geq 1}\left|a_{n} Z_{n}\right|^{2}}\right\|_{L^{p, \infty}\left(\mathbb{S}^{d}\right)} \leq C\left\|\sqrt{\sum_{n \geq 1}\left|a_{n} Z_{n}\right|^{2}} \times \mathbf{1}_{\left[0, \frac{\pi}{2}\right]}(\Theta)\right\|_{L^{p, \infty\left(\mathbb{S}^{d}\right)}} .
$$

Pour obtenir l'estimation (94) à partir de (95), on remarque d'une part l'inégalité triviale

$$
\left\|\sqrt{\sum_{n \geq 1}\left|a_{n} \widetilde{Z}_{n}\right|^{2}}\right\|_{L^{p, \infty}\left(\mathbb{S}^{d}\right)} \leq\left\|\sqrt{\sum_{n \geq 1}\left|a_{n} \widetilde{Z}_{n}\right|^{2}}\right\|_{L^{p}\left(\mathbb{S}^{d}\right)},
$$

d'autre part que la fonction $x \mapsto \sin (\Theta(x))^{-\frac{d}{p}}$ appartient à $L^{p, \infty}\left(\mathbb{S}^{d}\right)$. Cela peut se vérifier avec la formule de changement de variable (89) mais il est plus simple de remarquer que sur un voisinage $\mathcal{V}$ du pôle $(1,0, \ldots 0) \in$ $\mathbb{S}^{d}$ nous avons l'équivalent $\sin (\Theta)^{-\frac{d}{p}} \sim \Theta^{-\frac{d}{p}}$ et que la mesure de $\mathbb{S}^{d}$ sur $\mathcal{V}$ est comparable à la mesure de Lebesgue d'un voisinage de l'origine de $\mathbb{R}^{d}$.

Si l'on essaie d'estimer directement la norme dans $L^{p}\left(\mathbb{S}^{d}\right)$ de $\sqrt{\sum_{n \geq 1}\left|a_{n} Z_{n}\right|^{2}}$ en comparant $Z_{n}$ avec $\widetilde{Z}_{n}$ à l'aide de (92) et (93), alors la preuve précédente montre que l'on commet une perte avec l'inégalité de Hölder. 
De façon précise, après application de l'inégalité de Hölder, la formule (99) et le contrôle de $\left\|A_{2}(\Theta)\right\|_{L^{p}\left(\mathbb{S}^{d}\right)}$ conduisent aux inégalités

$$
\begin{aligned}
\left\|\sum_{n \geq 1} n^{\frac{d}{p}}\left(\sum_{k>n} \frac{S_{k}^{\frac{p}{2}}}{k^{d+1}}\right)^{\frac{1}{p}} \mathbf{1}_{\left.\frac{c}{n+1}, \frac{c}{n}\right]}(\Theta)\right\|_{L^{p}\left(\mathbb{S}^{d}\right)}^{p} & \simeq_{d, p} \sum_{n \geq 1} \frac{n^{\frac{d}{p} p}}{n^{d+1}} \sum_{k>n} \frac{S_{k}^{\frac{p}{2}}}{k^{d+1}} \\
& \simeq_{d, p} \sum_{n \geq 1} \frac{1}{n} \sum_{k>n} \frac{S_{k}^{\frac{p}{2}}}{k^{d+1}} \\
& \simeq d, p \sum_{k \geq 1} \frac{\ln (k+1)}{k^{d+1}} S_{k}^{\frac{p}{2}} .
\end{aligned}
$$

Par comparaison avec (97), l'estimation précédente est mauvaise en raison du terme logarithmique. Un argument d'interpolation réelle bien connu va nous permettre de corriger le facteur logarithmique. C'est maintenant que le théorème 2.5 intervient via le lemme suivant.

Lemme 3.11. - La famille d'espaces de Banach $\left(\mathbf{P L}^{p}\left(\mathbb{S}^{d}, \oplus \mathbb{C} \widetilde{Z}_{n}\right)\right)_{p \in] 1,+\infty[}$ est stable par interpolation réelle au sens du théorème 2.5 .

PREUVE. Il s'agit d'appliquer le théorème 2.5 avec $E_{n}=\mathbb{C} \widetilde{Z}_{n}$ et $\sqrt{e_{n}(x)}=\frac{\left|\widetilde{Z}_{n}(x)\right|}{\left\|\widetilde{Z}_{n}\right\|_{L^{2}\left(\mathbb{S}^{d}\right)}}$. D'une part, on a le comportement asymptotique

$$
\forall p>1 \quad\left\|\widetilde{Z}_{n}\right\|_{L^{p}\left(\mathbb{S}^{d}\right)} \simeq_{d, p} n^{\frac{d-1}{2}-\frac{d}{p}}
$$

ce qui nous assure la validité de (36). Quant à l'hypothèse (35), on peut écrire d'après (92) :

$$
\forall x \in \mathbb{S}^{d} \quad \sup _{n \geq 1} \frac{\left|\widetilde{Z}_{n}(x)\right|}{\left\|\widetilde{Z}_{n}\right\|_{L^{p}\left(\mathbb{S}^{d}\right)}} \leq C(d, p) \sup _{n \geq 1} \frac{\left|\widetilde{Z}_{n}(x)\right|}{n^{\frac{d-1}{2}-\frac{d}{p}}}=C(d, p) \sup _{n \geq 1} n^{\frac{d}{p}} \mathbf{1}_{\left[0, \frac{c}{n}\right]}(\Theta) \leq \frac{C(d, p)}{\sin (\Theta)^{\frac{d}{p}}} .
$$

Or l'on a remarqué à la fin de la proposition 3.10 que la fonction $x \mapsto \sin (\Theta)^{-\frac{d}{p}} \operatorname{appartient}$ à $L^{p, \infty}\left(\mathbb{S}^{d}\right)$.

Nous sommes en mesure d'obtenir la partie de la proposition 3.1 qui décrit $\mathbf{P} \mathbf{L}^{p}\left(\mathbb{S}^{d}, \oplus \mathbb{C} Z_{n}\right)$.

Proposition 3.12. - Pour tout réel $p>\frac{2 d}{d-1}$, et pour toute suite complexe $\left(a_{n}\right)_{n \geq 1}$ on a

$$
\left\|\sqrt{\sum_{n \geq 1}\left|a_{n} \widetilde{Z}_{n}\right|^{2}}\right\|_{L^{p}\left(\mathbb{S}^{d}\right)} \simeq d,\left\|\sqrt{\sum_{n \geq 1}\left|a_{n} Z_{n}\right|^{2}}\right\|_{L^{p}\left(\mathbb{S}^{d}\right)} .
$$

En outre, on a

$$
\left\|\sqrt{\sum_{n \geq 1}\left|a_{n} Z_{n}\right|^{2}}\right\|_{L^{p}\left(\mathbb{S}^{d}\right)} \simeq_{d, p}\left[\sum_{n \geq 1} \frac{1}{n^{d+1}}\left(\sum_{k=1}^{n} k^{d-1}\left|a_{k}\right|^{2}\right)^{\frac{p}{2}}\right]^{\frac{1}{p}}
$$

Preuve. L'équivalence (102) découlera de (97) et (101). Montrons donc (101). L'inégalité $\left|\widetilde{Z}_{n}\right| \leq\left|Z_{n}\right|$ donne un sens de l'équivalence (101). L'autre sens équivaut à la bornitude de l'opérateur :

$$
\begin{aligned}
\mathbf{P L}^{p}\left(\mathbb{S}^{d}, \oplus \mathbb{C} \widetilde{Z}_{n}\right) & \rightarrow \quad L^{p}\left(\mathbb{S}^{d}, \ell^{2}\left(\mathbb{N}^{\star}\right)\right) \\
\left(a_{n} \widetilde{Z}_{n}\right)_{n \geq 1} & \mapsto \quad\left(a_{n} Z_{n}\right)_{n \geq 1} .
\end{aligned}
$$

L'inégalité (95) de la proposition 3.10 montre, pour tout $p>\frac{2 d}{d-1}$, la continuité de l'opérateur

$$
\begin{aligned}
\mathbf{P L}^{p}\left(\mathbb{S}^{d}, \oplus \mathbb{C} \widetilde{Z}_{n}\right) & \rightarrow L^{p, \infty}\left(\mathbb{S}^{d}, \ell^{2}\left(\mathbb{N}^{\star}\right)\right) \\
\left(a_{n} \widetilde{Z}_{n}\right)_{n \geq 1} & \mapsto \quad\left(a_{n} Z_{n}\right)_{n \geq 1} .
\end{aligned}
$$

À présent, il s'agit de raisonner par interpolation réelle. Fixons deux réels $p_{1}^{\prime}$ et $p_{2}^{\prime}$ tels que

$$
\frac{2 d}{d-1}<p_{1}^{\prime}<p<p_{2}^{\prime}
$$


Considérons de plus l'unique réel $\theta^{\prime} \in[0,1]$ tel que

$$
\frac{1}{p}=\frac{1-\theta^{\prime}}{p_{1}^{\prime}}+\frac{\theta^{\prime}}{p_{2}^{\prime}}
$$

Par application de la méthode d'interpolation réelle $[\cdot, \cdot]_{\theta^{\prime}, p}$, l'opérateur suivant est borné :

$$
\begin{aligned}
{\left[\mathbf{P L}^{p_{1}^{\prime}}\left(\mathbb{S}^{d}, \oplus \mathbb{C} \widetilde{Z}_{n}\right), \mathbf{P L}^{p_{2}^{\prime}}\left(\mathbb{S}^{d}, \oplus \mathbb{C} \widetilde{Z}_{n}\right)\right]_{\theta^{\prime}, p} } & \rightarrow\left[L_{x}^{p_{1}^{\prime}, \infty}\left(\mathbb{S}^{d}, \ell^{2}\left(\mathbb{N}^{\star}\right)\right), L^{p_{2}^{\prime}, \infty}\left(\mathbb{S}^{d}, \ell^{2}\left(\mathbb{N}^{\star}\right)\right)\right]_{\theta^{\prime}, p} \\
\left(a_{n} \widetilde{Z}_{n}\right)_{n \geq 1} & \mapsto\left(a_{n} Z_{n}\right)_{n \geq 1} .
\end{aligned}
$$

L'espace de départ s'identifie à $\mathbf{P} \mathbf{L}^{p}\left(\mathbb{S}^{d}, \oplus \mathbb{C} \widetilde{Z}_{n}\right)$ d'après le lemme 3.11. Quant à l'espace d'arrivée, il s'identifie à $L_{x}^{p}\left(\mathbb{S}^{d}, \ell^{2}\left(\mathbb{N}^{\star}\right)\right)$ d'après le théorème 2.32. Cela prouve (101).

En apparence, on n'a pas montré l'interpolation des espaces $\mathbf{P} \mathbf{L}^{p}\left(\mathbb{S}^{d}, \oplus \mathbb{C} Z_{n}\right)$. La preuve de la proposition suivante montre en fait que cela est inclus dans l'inégalité (94).

Proposition 3.13. - Les espaces $\mathbf{P} \mathbf{L}^{p}\left(\mathbb{S}^{d}, \oplus \mathbb{C} Z_{n}\right)$ sont stables par interpolation réelle et complexe pour $p$ parcourant $] \frac{2 d}{d-1},+\infty[$ au sens du théorème 2.5 .

Preuve. On peut donner deux preuves. La proposition 3.12 montre que les espaces $\mathbf{P L}^{p}\left(\mathbb{S}^{d}, \oplus \mathbb{C} Z_{n}\right)$ et $\mathbf{P L}^{p}\left(\mathbb{S}^{d}, \oplus \mathbb{C} \widetilde{Z}_{n}\right)$ sont isomorphes pour tout $p>\frac{2 d}{d-1}$. Le lemme 3.11 , qui contient aussi dans sa preuve l'interpolation complexe, donne alors la conclusion.

La seconde preuve utilise aussi la démonstration du lemme 3.11 avec l'argument additionnel suivant. D'après (94), on a pour tout $x \in \mathbb{S}^{d}$ vérifiant $\Theta(x) \in\left[0, \frac{\pi}{2}\right]$ :

$$
\left|Z_{n}(x)\right| \leq C(d, p)\left|\widetilde{Z}_{n}(x)\right|+\frac{C(d, p)}{\sin ^{\frac{d}{p}}(\Theta)}\left\|\widetilde{Z}_{n}\right\|_{L^{p}\left(\mathbb{S}^{d}\right)} .
$$

La symétrie $\Theta(x) \leftrightarrow \pi-\Theta(x)$ et le fait que $\sin ^{-d}(\Theta)$ appartient à $L^{1, \infty}\left(\mathbb{S}^{d}\right)$ nous donnent

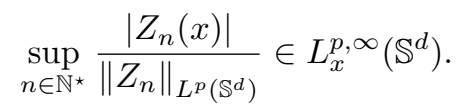

On conclut par application du théorème 2.5 pour les espaces $\mathbf{P} \mathbf{L}^{p}\left(\mathbb{S}^{d}, \oplus \mathbb{C} Z_{n}\right)$.

\section{Espaces $\mathbf{P L}^{p}$ pour l'oscillateur harmonique sur $\mathbb{R}^{d}$}

4.1. Reformulation des énoncés. - On reprend les notations de la partie 1.3. En particulier les résultats énoncés sont valides uniquement en dimension $d \geq 2$. Pour tout $p \in[1,+\infty[$, l'étude de l'espace $\mathbf{P} \mathbf{L}^{p}\left(\mathbb{R}^{d}, \oplus E_{d, n}\right)$ passe par la compréhension de la localisation des fonctions

$$
\begin{aligned}
e_{d, n}(x) & :=\sup \left\{\left|u_{n}(x)\right|^{2}, \quad u_{n} \in E_{d, n}, \quad\left\|u_{n}\right\|_{L^{2}\left(\mathbb{R}^{d}\right)}=1\right\} \\
& =\sum_{\substack{\left(i_{1}, \ldots, i_{d}\right) \in \mathbb{N}^{d} \\
i_{1}+\cdots+i_{d}=n}} h_{i_{1}}\left(x_{1}\right)^{2} \ldots h_{i_{d}}\left(x_{d}\right)^{2} .
\end{aligned}
$$

Suivant l'idée selon laquelle seule la concentration de $e_{d, n}$ devrait être significative, on démontre le résultat suivant.

Proposition 4.1. - Pour tout entier $n \in \mathbb{N}^{\star}$ et tout vecteur $x \in \mathbb{R}^{d}$ on a

$$
\begin{aligned}
& |x| \leq \sqrt{2(2 n+1)} \quad \Rightarrow \quad e_{d, n}(x) \leq C(d) n^{\frac{d}{2}-1} \\
& |x| \geq \sqrt{2(2 n+1)} \quad \Rightarrow \quad e_{d, n}(x) \leq C(d) e^{-\frac{|x|^{2}}{C(d)}} .
\end{aligned}
$$

Il existe aussi une constante universelle $\alpha \in] 0,1\left[\right.$ et un entier $n(d) \in \mathbb{N}^{\star}$ tels que pour tout entier $n \geq n(d)$ on a, quitte à augmenter $C(d) \geq 1$, l'implication

$$
\frac{C(d)}{\sqrt{2 n+1}} \leq|x| \leq \alpha \sqrt{2 n+1} \quad \Rightarrow \quad \frac{n^{\frac{d}{2}-1}}{C(d)} \leq e_{d, n}(x) \leq C(d) n^{\frac{d}{2}-1} .
$$


On justifiera plus loin que la fonction $x \mapsto e_{d, n}(x)$ est invariante par rotation autour de 0 , si bien que l'on a $e_{d, n}(x)=e_{d, n}((|x|, 0, \ldots, 0))$. À titre d'exemple, on examine les graphes pour $d=2$ et $n \in\{5,10,50\}$ de $e_{d, n}(x)$ en fonction de $|x| \in\left[0, \frac{3}{2} \sqrt{2 n+1}\right]$.
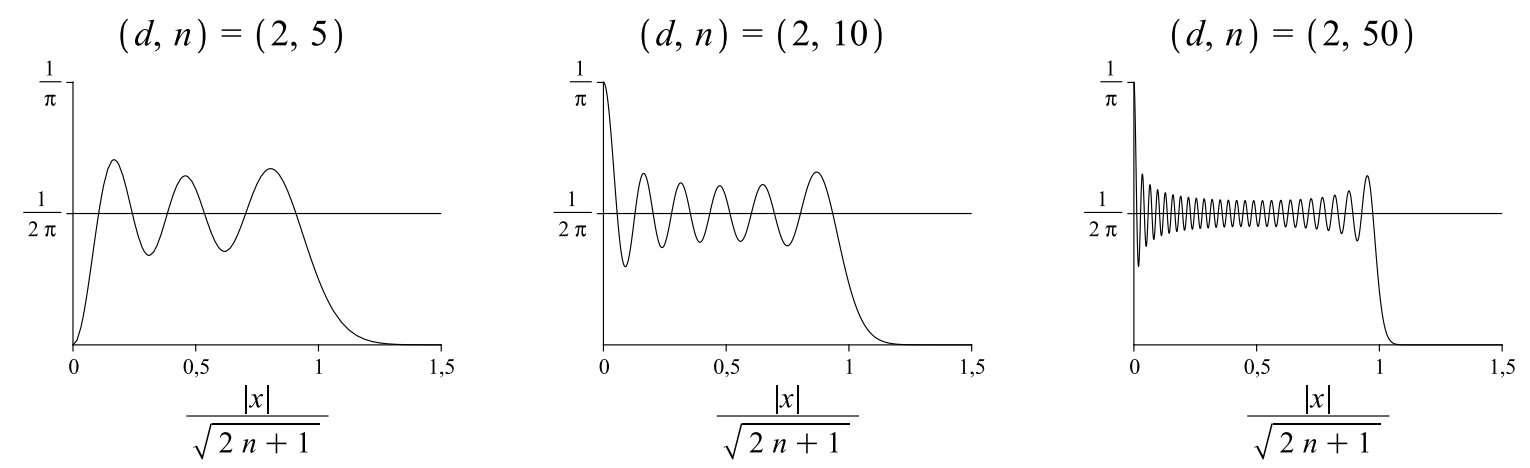

La majoration (104) est grossière et est obtenue grâce à des majorations classiques des fonctions de Hermite. Quant à la minoration de (105), sa démonstration est plus subtile et utilise des approximations essentiellement optimales des fonctions de Hermite dues à Muckhenhoupt. On notera que nous sommes obligés d'éviter, en toute rigueur, un voisinage de l'origine pour effectuer une minoration uniforme de $e_{d, n}$. En effet, pour tout entier $n$ impair la fonction $h_{n}$ est impaire et donc $e_{d, n}(0)$ est nul.

Après la rédaction de cet article, Didier Robert nous a signalé le lemme 10 de l'article [28] dans lequel se trouvent des estimations plus précises si $|x|$ appartient à un compact de la forme $[a \sqrt{2 n+d}, b \sqrt{2 n+d}]$ avec $0<a<b<1$. Par souci de comparaison, on se permet d'écrire cette approximation :

$$
e_{d, n}(x)=\frac{\mu_{d-1}\left(\mathbb{S}^{d-1}\right)}{(2 \pi)^{d}}\left(2 n+d-|x|^{2}\right)^{\frac{d}{2}-1}\left(1+\mathcal{O}_{a, b}\left(\frac{1}{2 n+d}\right)\right)
$$

où $\mathcal{O}_{a, b}$ est uniforme en la condition $a \leq \frac{|x|}{\sqrt{2 n+d}} \leq b$. Il s'agit d'une loi de Weyl locale pour l'oscillateur harmonique. Dans la théorie des espaces de Lebesgue probabilistes, il apparaîtra que l'on peut en fait considérer, en première approximation, que la fonction $e_{d, n}$ se localise uniformément sur le compact $\overline{\mathbb{B}_{d}(0, \sqrt{2 n+1})}$ avec une amplitude d'ordre $n^{\frac{d}{2}-1}$.

Une application immédiate de la proposition 4.1 est donnée par les estimations

$$
\forall p \in\left[1,+\infty\left[\cup\{+\infty\} \quad \forall n \in \mathbb{N}^{\star} \quad\left\|\sqrt{e_{d, n}}\right\|_{L^{p}\left(\mathbb{R}^{d}\right)} \simeq_{d, p} n^{\frac{d-1}{2}-\frac{d}{2}\left(\frac{1}{2}-\frac{1}{p}\right)}=n^{\frac{1}{2}\left[\frac{d}{2}-1+\frac{d}{p}\right]}\right.\right.
$$

La majoration $\left\|e_{d, n}\right\|_{L^{\frac{p}{2}}\left(\mathbb{R}^{d}\right)} \lesssim_{d, p} n^{\frac{d}{2}-1+\frac{d}{p}}$ est connue pour $p \geq 2$ et est généralement traitée par interpolation entre $p=2$ et $p=+\infty$, mais nous ne connaissons pas de référence où l'optimalité est prouvée (voir [47, Lemma 3.5] et les références indiquées). Les hypothèses du théorème 2.6 de dualité sont alors très faciles à vérifier. D’une part, (13) nous donne

$$
\forall p \in] 1,+\infty\left[\quad\left\|\sqrt{e_{d, n}}\right\|_{L^{p}\left(\mathbb{R}^{d}\right)}\left\|\sqrt{e_{d, n}}\right\|_{L^{\frac{p}{p-1}}\left(\mathbb{R}^{d}\right)} \lesssim d, p \operatorname{dim}\left(E_{d, n}\right)\right.
$$


D'autre part, en utilisant (104) et (106), on a

$$
\begin{aligned}
& \forall n \in \mathbb{N}^{\star} \frac{\sqrt{e_{d, n}(x)}}{\left\|\sqrt{e_{d, n}}\right\|_{L^{p}\left(\mathbb{R}^{d}\right)}} \lesssim_{d, p} \frac{1}{n^{\frac{d}{2 p}}} \mathbf{1}_{\mathbb{B}_{d}(0, \sqrt{2(2 n+1)})}(x)+\frac{e^{-\frac{|x|^{2}}{C(d)}}}{n^{\frac{1}{2}\left(\frac{d}{2}-1+\frac{d}{p}\right)}} \\
& \sup _{n \geq 1} \frac{\sqrt{e_{d, n}(x)}}{\left\|\sqrt{e_{d, n}}\right\|_{L^{p}\left(\mathbb{R}^{d}\right)}} \lesssim_{d, p} \frac{1}{|x|^{\frac{d}{p}}}+e^{-\frac{|x|^{2}}{C(d)}} \\
& \sup _{n \geq 0} \frac{\sqrt{e_{d, n}}}{\left\|\sqrt{e_{d, n}}\right\|_{L^{p}\left(\mathbb{R}^{d}\right)}} \in L^{p, \infty}\left(\mathbb{R}^{d}\right) .
\end{aligned}
$$

Ainsi, on sait par avance que les espaces $\mathbf{P} \mathbf{L}^{p}\left(\mathbb{R}^{d}, \oplus E_{d, n}\right)$ sont stables par dualité. Tout comme pour les harmoniques sphériques, ces espaces vont s'identifier à des sous-espaces de distributions sur $\mathbb{R}^{d}$. Pour le voir, commençons par rappeler la définition des espaces de Sobolev naturellement associés à l'oscillateur harmonique. En notant $\Pi_{n}: L^{2}\left(\mathbb{R}^{d}\right) \rightarrow L^{2}\left(\mathbb{R}^{d}\right)$ le projecteur orthogonal sur $E_{d, n}=\operatorname{ker}\left(-\Delta+|x|^{2}-2 n+d\right)$, on a :

$$
\begin{aligned}
\forall s \geq 0 \quad \mathcal{H}^{s}\left(\mathbb{R}^{d}\right) & :=\operatorname{Dom}\left(\left(-\Delta+|x|^{2}\right)^{\frac{s}{2}}\right) \\
& =\left\{u \in L^{2}\left(\mathbb{R}^{d}\right), \quad\left(-\Delta+|x|^{2}\right)^{\frac{s}{2}} u \in L^{2}\left(\mathbb{R}^{d}\right)\right\} \\
& =\left\{u \in L^{2}\left(\mathbb{R}^{d}\right), \quad \sum_{n \in \mathbb{N}}(1+n)^{s}\left\|\Pi_{n}(u)\right\|_{L^{2}\left(\mathbb{R}^{d}\right)}^{2}<+\infty\right\}
\end{aligned}
$$

Rappelons aussi que cet espace abstrait admet, si $s \in \mathbb{N}$, la caractérisation fonctionnelle suivante (voir la preuve de [61, Lemma 2.4] et [30, page 2765]) :

$$
\mathcal{H}^{s}\left(\mathbb{R}^{d}\right)=\left\{u \in L^{2}\left(\mathbb{R}^{d}\right), \forall\left(m_{0}, m_{1}, \ldots, m_{d}\right) \in \mathbb{N}^{d}, \quad m_{0}+\cdots+m_{d} \leq s \Rightarrow|x|^{m_{0}} \partial_{x_{1}}^{m_{1}} \ldots \partial_{x_{d}}^{m_{d}} u \in L^{2}\left(\mathbb{R}^{d}\right)\right\}
$$

Toute fonction $\varphi$ de l'espace de Schwartz $\mathcal{S}\left(\mathbb{R}^{d}\right)=\bigcap_{s \in \mathbb{N}} \mathcal{H}^{s}\left(\mathbb{R}^{d}\right)$ admet alors une décomposition

$$
\varphi=\sum_{n \geq 0} \Pi_{n}(\varphi), \quad \forall \alpha>0 \quad \forall n \in \mathbb{N}^{\star} \quad\left\|\Pi_{n}(\phi)\right\|_{L^{2}\left(\mathbb{R}^{d}\right)} \leq \frac{C(\alpha)}{n^{\alpha}} .
$$

On en déduit par dualité que toute distribution tempérée $u \in \mathcal{S}^{\prime}\left(\mathbb{R}^{d}\right)$ admet une décomposition en série faiblement convergente pour la dualité $\left(\mathcal{S}\left(\mathbb{R}^{d}\right), \mathcal{S}^{\prime}\left(\mathbb{R}^{d}\right)\right)$ :

$$
u=\sum_{n \in \mathbb{N}} \Pi_{n}(u), \quad \exists \alpha>0 \quad \forall n \in \mathbb{N}^{\star} \quad\left\|\Pi_{n}(u)\right\|_{L^{2}\left(\mathbb{R}^{d}\right)} \leq C(\alpha) n^{\alpha} .
$$

Cela nous amène à définir des espaces de Sobolev pour tout $s \in \mathbb{R}$

$$
\forall s \in \mathbb{R} \quad \mathcal{H}^{s}(\mathbb{R})=\left\{u \in \mathcal{S}^{\prime}\left(\mathbb{R}^{d}\right), \quad \sum_{n \in \mathbb{N}}(1+n)^{s}\left\|\Pi_{n}(u)\right\|_{L^{2}\left(\mathbb{R}^{d}\right)}^{2}<+\infty\right\} .
$$

Nous pouvons maintenant énoncer un lemme d'identification.

Lemme 4.2. - Pour tous $p \in\left[1,+\infty\left[\right.\right.$ et $\left(u_{n}\right)_{n \in \mathbb{N}} \in \mathbf{P L}^{p}\left(\mathbb{R}^{d}, \oplus E_{d, n}\right)$, la série $\sum u_{n}$ converge pour la dualité $\left(\mathcal{S}\left(\mathbb{R}^{d}\right), \mathcal{S}^{\prime}\left(\mathbb{R}^{d}\right)\right)$ vers une distribution tempérée. Par suite, on peut identifier $\mathbf{P} \mathbf{L}^{p}\left(\mathbb{R}^{d}, \oplus E_{d, n}\right)$ au sous-espace des distributions tempérées $u \in \mathcal{S}^{\prime}\left(\mathbb{R}^{d}\right)$ vérifiant

$$
\|u\|_{\mathbf{P L}^{p}\left(\mathbb{R}^{d}, \oplus E_{n}\right)}=\left\|\sqrt{\sum_{n \in \mathbb{N}}\left\|\Pi_{n}(u)\right\|_{L^{2}\left(\mathbb{R}^{d}\right)}^{2} \frac{e_{d, n}(x)}{\operatorname{dim}\left(E_{d, n}\right)}}\right\|_{L_{x}^{p}\left(\mathbb{R}^{d}\right)}<+\infty .
$$

Preuve. On invoque l'inégalité triviale :

$$
\|u\|_{\mathbf{P L}^{p}\left(\mathbb{R}^{d}, \oplus E_{d, n}\right)} \geq \sup _{n \in \mathbb{N}} \frac{\left\|u_{n}\right\|_{L^{2}\left(\mathbb{R}^{d}\right)}}{\sqrt{\operatorname{dim}\left(E_{d, n}\right)}}\left\|\sqrt{e_{d, n}}\right\|_{L^{p}\left(\mathbb{R}^{d}\right)} .
$$

Les équivalents (13) et (106) assurent que $\left(\left\|u_{n}\right\|_{L^{2}\left(\mathbb{R}^{d}\right)}\right)_{n \in \mathbb{N}}$ est à croissance polynomiale. 
La proposition 4.1, le théorème 2.6 de dualité et le théorème 2.5 d'interpolation vont nous permettre de décrire complètement les sous-espaces $\mathbf{P L}^{p}\left(\mathbb{R}^{d}, \oplus E_{d, n}\right)$ et leurs propriétés de dualité et d'interpolation pour $p \in] 1,+\infty[$. Cela nous donne l'équivalence i) $\Leftrightarrow$ ii) du théorème 1.4.

Théorème 4.3. - Pour tout réel $p \in\left[1,+\infty\left[\right.\right.$ et toute distribution tempérée $u \in \mathcal{S}^{\prime}\left(\mathbb{R}^{d}\right)$, on a

$$
\|u\|_{\mathbf{P L}^{p}\left(\mathbb{R}^{d}, \oplus E_{d, n}\right)} \simeq_{d, p}\left\|\Pi_{0}(u)\right\|_{L^{2}\left(\mathbb{R}^{d}\right)}+\left[\sum_{n \geq 1} n^{\frac{d}{2}-1}\left(\sum_{k \geq n} \frac{\left\|\Pi_{k}(u)\right\|_{L^{2}\left(\mathbb{R}^{d}\right)}^{2}}{k^{\frac{d}{2}}}\right)^{\frac{p}{2}}\right]^{\frac{1}{p}} .
$$

En outre, nous avons les propriétés de dualité et d'interpolation :

i) pour tout $p \in] 1,+\infty\left[\right.$, on pose $q=\frac{p}{p-1}$ l'exposant conjugué. L'injection canonique

$$
\Lambda_{p}: \mathbf{P} \mathbf{L}^{q}\left(\mathbb{R}^{d}, \oplus E_{d, n}\right) \rightarrow \mathbf{P L}^{p}\left(\mathbb{R}^{d}, \oplus E_{d, n}\right)^{\prime}
$$

qui à un élément $w \in \mathbf{P L}^{q}\left(\mathbb{R}^{d}, \oplus E_{d, n}\right)$ associe la forme linéaire

$$
u \in \mathbf{P L}^{p}\left(\mathbb{R}^{d}, \oplus E_{d, n}\right) \mapsto \sum_{n \geq 0}\left\langle\Pi_{n}(u), \Pi_{n}(w)\right\rangle_{L^{2}\left(\mathbb{R}^{d}\right)}
$$

est bien définie et est un isomorphisme d'espaces de Banach.

ii) les espaces $\left(\mathbf{P L}^{p}\left(\mathbb{R}^{d}, \oplus E_{d, n}\right)\right)_{p \in] 1,+\infty[}$ sont stables par interpolation complexe et réelle au sens du théorème 2.5.

Le théorème précédent ressemble aux propositions 3.1 et 3.2 mais sa preuve est bien plus simple car la concentration des fonctions $e_{d, n}$ est bien meilleure que celle des harmoniques sphériques $Y_{n}$ et $Z_{n}$ étudiées dans la partie 3. En effet, la proposition 4.1 assure que l'on peut brutalement contrôler $e_{d, n}(x)$ par le terme gaussien $e^{-\frac{|x|^{2}}{C(d)}} \ll 1$ à l'extérieur de la boule $\mathbb{B}_{d}(0, \sqrt{2(2 n+1)})$.

L'équivalence de normes (110) implique déjà quelques faits non triviaux :

- pour tout $p \in\left[1,+\infty\left[\right.\right.$ on a l'inclusion $\mathbf{P L}^{p}\left(\mathbb{R}^{d}, \oplus E_{d, n}\right) \subset \mathcal{H}^{-\frac{d}{2}}\left(\mathbb{R}^{d}\right)$. Cela signifie qu'il faut un minimum de régularité pour espérer arriver presque sûrement dans $L^{p}\left(\mathbb{R}^{d}\right)$.

- pour tous réels $p_{1}<p_{2}$ on a l'inclusion stricte $\mathbf{P L}^{p_{1}}\left(\mathbb{R}^{d}, \oplus E_{d, n}\right) \subset \mathbf{P L}^{p_{2}}\left(\mathbb{R}^{d}, \oplus E_{d, n}\right)$. D'une part, cela contraste fortement avec le fait que $L^{p_{1}}\left(\mathbb{R}^{d}\right)$ n'est pas inclus dans $L^{p_{2}}\left(\mathbb{R}^{d}\right)$. D'autre part, la conclusion du théorème de Paley-Zygmund est vérifiée pour l'oscillateur harmonique (choisir $p_{1}=2$ ).

Il est temps à présent d'énoncer les injections de Sobolev probabilistes de l'oscillateur harmonique multidimensionnel (ce qui donne la fin du théorème 1.4).

Théorème 4.4. - Pour tout réel $p \in] 2,+\infty\left[\right.$, on note $\left.q=\frac{p}{p-1} \in\right] 1,2[$. Nous avons les inclusions

$$
\begin{aligned}
& \mathcal{H}^{-d\left(\frac{1}{2}-\frac{1}{p}\right)}\left(\mathbb{R}^{d}\right) \quad \subset \quad \mathbf{P L}^{p}\left(\mathbb{R}^{d}, \oplus E_{d, n}\right) \subset \bigcap_{\varepsilon>0} \mathcal{H}^{-d\left(\frac{1}{2}-\frac{1}{p}\right)-\varepsilon}\left(\mathbb{R}^{d}\right), \\
& \bigcup_{\varepsilon>0} \mathcal{H}^{d\left(\frac{1}{q}-\frac{1}{2}\right)+\varepsilon}\left(\mathbb{R}^{d}\right) \quad \subset \quad \mathbf{P L}^{q}\left(\mathbb{R}^{d}, \oplus E_{d, n}\right) \quad \subset \quad \mathcal{H}^{d\left(\frac{1}{q}-\frac{1}{2}\right)}\left(\mathbb{R}^{d}\right) .
\end{aligned}
$$

Remarquons que pour tout $q \in[1,2]$, l'espace $\mathbf{P} \mathbf{L}^{q}\left(\mathbb{R}^{d}, \oplus E_{d, n}\right) \subset L^{2}\left(\mathbb{R}^{d}\right)$ est un espace de fonctions (alors que pour les harmoniques sphériques $Y_{n}$, l'espace $\mathbf{P} \mathbf{L}^{q}\left(X, \oplus Y_{n}\right)$ est en général constitué de distributions). En choisissant $m_{0}=0$ dans (109), on voit que l'espace de Sobolev $\mathcal{H}^{s}\left(\mathbb{R}^{d}\right)$ est inclus dans l'usuel espace de Sobolev $H^{s}\left(\mathbb{R}^{d}\right)$. Dans le cas $p>2$, les injections de Sobolev déterministes (17) s'écrivent alors

$$
\mathcal{H}^{d\left(\frac{1}{2}-\frac{1}{p}\right)}\left(\mathbb{R}^{d}\right) \subset H^{d\left(\frac{1}{2}-\frac{1}{p}\right)}\left(\mathbb{R}^{d}\right) \subset L^{p}\left(\mathbb{R}^{d}\right) .
$$

En autorisant un aléa pour arriver dans $L^{p}\left(\mathbb{R}^{d}\right)$, l'injection de Sobolev probabiliste $\mathcal{H}^{-d\left(\frac{1}{2}-\frac{1}{p}\right)}\left(\mathbb{R}^{d}\right) \subset$ $\mathbf{P L}^{p}\left(\mathbb{R}^{d}, \oplus E_{d, n}\right)$ assure donc presque sûrement un gain de $2 d\left(\frac{1}{2}-\frac{1}{p}\right)$ dérivées. 
4.2. Rappels sur les fonctions de Hermite. - Par récurrence, on peut simplifier (103) en

$$
e_{d, n}(x)=\sum_{\substack{\left(i_{1}, \ldots, i_{d}\right) \in \mathbb{N}^{d} \\ i_{1}+\cdots+i_{d}=n}} h_{i_{1}}\left(x_{1}\right)^{2} \ldots h_{i_{d}}\left(x_{d}\right)^{2}=\sum_{k=0}^{n} e_{d-1, k}\left(x_{1}, \ldots, x_{d-1}\right) h_{n-k}\left(x_{d}\right)^{2} .
$$

Cela dit, nous allons utiliser d'autres formules plus maniables. Remarquons que toute rotation linéaire de $\mathbb{R}^{d}$ commute avec l'oscillateur harmonique $-\Delta+|x|^{2}$ et laisse donc invariant son sous-espace propre $E_{d, n}$ associé à la valeur propre $d+2 n$. On en déduit que la fonction $e_{d, n}$ de $E_{d, n}$ ne dépend que de la norme euclidienne $|x|=\sqrt{x_{1}^{2}+\cdots+x_{d}^{2}}$ et l'on peut écrire

$$
\begin{aligned}
e_{d, n}(x) & =\sum_{k=0}^{n} e_{d-1, k}(\overbrace{|x|, 0, \ldots, 0}^{\in \mathbb{R}^{d-1}}) h_{n-k}(0)^{2} \\
& =\sum_{k=0}^{n} e_{d-1, k}(\overbrace{0, \ldots, 0}^{\in \mathbb{R}^{d-1}}) h_{n-k}(|x|)^{2} .
\end{aligned}
$$

Le résultat suivant nous donne des estimations faciles en 0.

Proposition 4.5. - Pour tout entier $k \in \mathbb{N}$, on a $h_{2 k+1}(0)=0$ et l'équivalence $(-1)^{k} h_{2 k}(0) \simeq \frac{1}{(k+1)^{1 / 4}}$. Pour tout entier $n \in \mathbb{N}$, on a

$$
\begin{aligned}
n \in 2 \mathbb{N}+1 & \Rightarrow \quad e_{d, n}(0)=0 \\
n \in 2 \mathbb{N} & \Rightarrow \quad e_{d, n}(0) \simeq_{d} n^{\frac{d}{2}-1}
\end{aligned}
$$

Preuve. La première équivalence découle de la formule (5.5.5) de [54] :

$$
(-1)^{k} h_{2 k}(0)=(-1)^{k} \frac{H_{2 k}(0)}{\sqrt{(2 k) ! 4^{k} \sqrt{\pi}}}=\frac{\sqrt{(2 k) !}}{k ! 2^{k} \pi^{1 / 4}} \sim \frac{1}{\sqrt{\pi} \sqrt[4]{k}} .
$$

Pour le cas $n$ impair, on a déjà remarqué l'égalité $e_{d, n}(0)=0$ après l'énoncé de la proposition 4.1. Pour le cas $n$ pair, on peut écrire :

$$
\begin{aligned}
& e_{d, n}(0)=\sum_{2 i_{1}+\cdots+2 i_{d}=n} h_{2 i_{1}}(0)^{2} \cdots h_{2 i_{d}}(0)^{2} \\
& \simeq_{d} \sum_{2 i_{1}+\cdots+2 i_{d}=n} \frac{1}{\sqrt{\left(1+i_{1}\right) \cdots\left(1+i_{d}\right)}} \\
& \gtrsim_{d} \sum_{\substack{2 i_{1}+\cdots+2 i_{d}=n \\
n^{\frac{d}{2}-1}}} n^{-\frac{d}{2}}
\end{aligned}
$$

La majoration $e_{d, n}(0) \lesssim_{d} n^{\frac{d}{2}-1}$ peut se démontrer par récurrence sur $d$ en séparant les sommes suivantes selon que $\ell$ est plus petit ou plus grand que $\frac{n}{4}$ :

$$
e_{d, n}(0)=\sum_{\ell=0}^{\frac{n}{2}} e_{d-1, n-2 \ell}(0) h_{2 \ell}(0)^{2} \simeq \sum_{\ell=0}^{\frac{n}{2}} \frac{e_{d-1, n-2 \ell}(0)}{\sqrt{1+\ell}}
$$

Nous aurons besoin d'estimations précises concernant le comportement des fonctions de Hermite. Pour le résultat suivant, on fait référence à [56, Lemma 1.5.1] ou [43, line (2.3)].

Proposition 4.6. - Il existe deux constantes universelles $C \geq 1$ et $\gamma>0$ telles que pour tous $x \in \mathbb{R}$ et $n \in \mathbb{N}$ on a

$$
\begin{aligned}
& |x| \leq \sqrt{2(2 n+1)} \Rightarrow\left|h_{n}(x)\right| \leq \frac{C}{\sqrt[4]{\left|2 n+1-x^{2}\right|+\sqrt[3]{2 n+1}}} \\
& |x|>\sqrt{2(2 n+1)} \Rightarrow\left|h_{n}(x)\right| \leq C e^{-\gamma x^{2}}
\end{aligned}
$$


En particulier, on a

$$
|x| \leq \sqrt{2 n+1} \Rightarrow\left|h_{n}(x)\right| \leq \frac{C}{\sqrt[4]{2 n+2-x^{2}}}
$$

Comme les fonctions $h_{n}$ oscillent et s'annulent plusieurs fois, les estimations de la proposition 4.6 sont inutilisables pour minorer les fonctions $\left|h_{n}\right|$. Pour remédier à cela, on fait appel à un résultat d'approximation des fonctions de Hermite dû à Muckenhoupt et prouvé dans [42, line 2.4, page 421] à partir de [20, line 6.12, page 23].

Proposition 4.7. - Introduisons la fonction croissante $\Phi:[0,1] \rightarrow\left[0, \frac{\pi}{4}\right]$ définie par

$$
\Phi(u)=\int_{0}^{u} \sqrt{1-s^{2}} d s=\frac{1}{2} \arcsin (u)+\frac{1}{2} u \sqrt{1-u^{2}} .
$$

Il existe une constante universelle $C \geq 1$ telle que pour tous $n \in \mathbb{N}$ et $x \in\left[0, \sqrt{2 n+1}-(2 n+1)^{-\frac{1}{6}}\right]$ on a

$$
\left|h_{n}(x)-\frac{\sqrt{2}}{\sqrt{\pi}\left(2 n+1-x^{2}\right)^{\frac{1}{4}}} \cos \left[(2 n+1) \Phi\left(\frac{x}{\sqrt{2 n+1}}\right)-\frac{n \pi}{2}\right]\right| \leq \frac{C \sqrt{2 n+1}}{\left(2 n+1-x^{2}\right)^{\frac{7}{4}}} .
$$

Preuve. En fait, le terme principal est exprimé dans [42, line 2.4, page 421] sous la forme différente mais équivalente

$$
\frac{\sqrt{2}}{\sqrt{\pi}\left(2 n+1-x^{2}\right)^{\frac{1}{4}}} \cos \left[\frac{1}{4}(2 n+1)[2 \theta-\sin (2 \theta)]-\frac{\pi}{4}\right],
$$

$\operatorname{avec} \theta=\frac{\pi}{2}-\arcsin \left(\frac{x}{\sqrt{2 n+1}}\right)$ et donc $\sin (2 \theta)=2 \sin (\theta) \cos (\theta)=2 \frac{x}{\sqrt{2 n+1}} \sqrt{1-\frac{x^{2}}{2 n+1}}$.

Pour tout $\beta \in] 0,1[$ et $x \in[0, \beta \sqrt{2 n+1}]$, l'inégalité (113) et la proposition 4.7 nous amènent donc à

$$
\left|h_{n}(x)^{2}-\frac{2}{\pi \sqrt{2 n+1-x^{2}}} \cos ^{2}\left[(2 n+1) \Phi\left(\frac{x}{\sqrt{2 n+1}}\right)-\frac{n \pi}{2}\right]\right| \leq \frac{C(\beta)}{(2 n+1)^{\frac{3}{2}}} .
$$

C'est la formule précédente et la proposition 4.6 que nous allons utiliser pour comprendre la localisation spatiale de la fonction $e_{d, n}$ de l'oscillateur harmonique multidimensionnel.

4.3. Preuve de la proposition 4.1, majoration (104) de la fonction $e_{d, n}$. - À l'aide de [33, Corollary 3.2 , case $n \geq 2$ and $p=\infty, \lambda=\sqrt{2 n+d}]$, on a pour tout $x \in \mathbb{R}^{d}$

$$
\begin{aligned}
e_{d, n}(x) & =\sup \left\{\left|u_{n}(x)\right|^{2}, \quad u_{n} \in E_{d, n}, \quad\left\|u_{n}\right\|_{L^{2}\left(\mathbb{R}^{d}\right)}=1\right\} \\
& \leq \sup \left\{\left\|u_{n}\right\|_{L^{\infty}\left(\mathbb{R}^{d}\right)}^{2}, \quad u_{n} \in E_{d, n}, \quad\left\|u_{n}\right\|_{L^{2}\left(\mathbb{R}^{d}\right)}=1\right\} \\
& \leq C(d) n^{\frac{d}{2}-1} .
\end{aligned}
$$

Il nous reste à analyser le cas $|x|>\sqrt{2(2 n+1)}$. Nous ne traitons que le sous-cas $n \in 2 \mathbb{N}$ car le sous-cas $n \in 2 \mathbb{N}+1$ se traite de la même façon. La formule (112) et la proposition 4.5 donnent

$$
e_{d, n}(x)=\sum_{k=0}^{\frac{n}{2}} h_{2 k}(|x|)^{2} e_{d-1, n-2 k}(0) \simeq_{d} \sum_{k=0}^{\frac{n}{2}} h_{2 k}(|x|)^{2}(1+n-2 k)^{\frac{d}{2}-\frac{3}{2}} .
$$

Par suite, la proposition 4.6 nous donne

$$
e_{d, n}(x) \leq C(d) e^{-2 \gamma|x|^{2}} \sum_{k=0}^{\frac{n}{2}}(1+n-2 k)^{\frac{d}{2}-\frac{3}{2}} \leq C(d) n^{\frac{d}{2}-\frac{1}{2}} e^{-2 \gamma|x|^{2}} \leq C(d)|x|^{d-1} e^{-2 \gamma|x|^{2}}
$$

Quitte à augmenter $C(d) \geq 1$, le majorant précédent est majoré par $C(d) e^{\frac{-|x|^{2}}{C(d)}}$. 
4.4. Preuve de la proposition 4.1, minoration (105) de la fonction $e_{d, n}$. - Pour tout entier $d \geq 2$, on va démontrer par récurrence sur $d$ l'assertion

$$
H(d): \quad \forall \alpha \in] 0, \sin \left(\frac{1}{4}\right)\left[\exists C(d, \alpha)>1 \quad \liminf _{n \rightarrow+\infty}\left(\inf _{\frac{C(d, \alpha)}{\sqrt{2 n+1}} \leq|x| \leq \alpha \sqrt{2 n+1}} \frac{e_{d, n}(x)}{n^{\frac{d}{2}-1}}\right)>0 .\right.
$$

Dans toute cette preuve, on aura besoin d'un réel $\beta \in] \alpha, \sin (1 / 4)[$ et l'on choisit par simplicité

$$
\beta:=\frac{1}{2}\left(\alpha+\sin \left(\frac{1}{4}\right)\right) .
$$

Premier cas : $H(2)$ avec $n \in 2 \mathbb{N}$. Il s'agit du cas le plus technique. Tout d'abord, l'invariance radiale de $e_{2, n}$ et la proposition 4.5 donnent pour tout $x \in \mathbb{R}^{2}$

$$
e_{2, n}(x)=\sum_{k=0}^{\frac{n}{2}} h_{2 k}(|x|)^{2} h_{n-2 k}(0)^{2} \simeq \sum_{k=0}^{\frac{n}{2}} \frac{h_{2 k}(|x|)^{2}}{\sqrt{1+n-2 k}} .
$$

Afin de pouvoir exploiter la formule (114), nous avons besoin de considérer des indices $k$ du même ordre de grandeur que $n$. De façon précise, nous allons sélectionner les indices $k$ tels que $\frac{\alpha^{2}}{2 \beta^{2}} n \leq k \leq \frac{n}{2}$. Puisque l'on a $\beta>\alpha$, on déduit que l'on a

$$
\begin{aligned}
n & \leq \frac{2 \beta^{2}}{\alpha^{2}} k+\frac{1}{2}\left(\frac{\beta^{2}}{\alpha^{2}}-1\right) \\
2 n+1 & \leq \frac{\beta^{2}}{\alpha^{2}}(4 k+1) \\
\alpha \sqrt{2 n+1} & \leq \beta \sqrt{4 k+1} .
\end{aligned}
$$

En utilisant (114) et en imposant $|x| \leq \alpha \sqrt{2 n+1}$, il existe une constante $C(\alpha)>1$ telle que

$$
e_{2, n}(x) \gtrsim \sum_{\frac{\alpha^{2}}{2 \beta^{2}} n \leq k \leq \frac{n}{2}} \frac{1}{\sqrt{(1+n-2 k) k}} \cos ^{2}\left[(4 k+1) \Phi\left(\frac{|x|}{\sqrt{4 k+1}}\right)\right]-\sum_{\frac{\alpha^{2}}{2 \beta^{2}} n \leq k \leq \frac{n}{2}} \frac{C(\alpha)}{(4 k+1)^{\frac{3}{2}}(1+n-2 k)^{\frac{1}{2}}},
$$

ce qui se minore grossièrement par

$$
\frac{1}{C(\alpha) n} \sum_{\frac{\alpha^{2}}{2 \beta^{2}} n \leq k \leq \frac{n}{2}} \cos ^{2}\left[(4 k+1) \Phi\left(\frac{|x|}{\sqrt{4 k+1}}\right)\right]-\sum_{\frac{\alpha^{2}}{2 \beta^{2}} n \leq k \leq \frac{n}{2}} \frac{C(\alpha)}{(4 k+1)^{\frac{3}{2}}(1+n-2 k)^{\frac{1}{2}}} .
$$

Or on a immédiatement

$$
\sum_{\frac{\alpha^{2}}{2 \beta^{2}} n \leq k \leq \frac{n}{2}} \frac{C(\alpha)}{(4 k+1)^{\frac{3}{2}}(1+n-2 k)^{\frac{1}{2}}} \leq C(\alpha) \frac{\sqrt{n}}{n^{3 / 2}}=\frac{C(\alpha)}{n} .
$$

Notons à présent $\left\lceil\frac{\alpha^{2}}{2 \beta^{2}} n\right\rceil$ le plus petit entier supérieur ou égal à $\frac{\alpha^{2}}{2 \beta^{2}} n$ et $\left\lfloor\frac{n}{2}\right\rfloor$ le plus grand entier inférieur ou égal à $\frac{n}{2}$. Si l'on définit

$$
S(n, x, \alpha, \beta):=\sum_{\left\lceil\frac{\alpha^{2}}{2 \beta^{2}} n\right\rceil \leq k \leq\left\lfloor\frac{n}{2}\right\rfloor} \cos \left[2(4 k+1) \Phi\left(\frac{|x|}{\sqrt{4 k+1}}\right)\right],
$$

alors notre minoration se reformule en

$$
e_{2, n}(x) \gtrsim \alpha \frac{1}{4}\left(1-\frac{\alpha^{2}}{\beta^{2}}\right)+\frac{1}{2 n} S(n, x, \alpha, \beta)-\frac{C(\alpha)}{n} .
$$

L'estimation grossière $|S(n, x, \alpha, \beta)| \leq \frac{n}{2}\left(1-\frac{\alpha^{2}}{\beta^{2}}\right)$ ne suffit pas pour minorer $e_{2, n}(x)$. Nous allons raffiner cette estimation grâce à l'oscillation des termes. On va faire appel à la formule d'Euler-Maclaurin au rang 0 en posant

$$
\begin{aligned}
\forall t \in\left[\frac{\alpha^{2}}{2 \beta^{2}} n, \frac{1}{2} n\right] \quad a_{x}(t) & :=2(4 t+1) \Phi\left(\frac{|x|}{\sqrt{4 t+1}}\right)=2(4 t+1) \int_{0}^{\frac{|x|}{\sqrt{4 t+1}}} \sqrt{1-s^{2}} d s, \\
& a_{x}^{\prime}(t)=4 \arcsin \left(\frac{|x|}{\sqrt{4 t+1}}\right) \in[0,4 \arcsin (\beta)] .
\end{aligned}
$$


Cela nous permet de reformuler $S(n, x, \alpha, \beta)$ en

$$
\frac{\cos \left[a_{x}\left(\left\lceil\frac{\alpha^{2}}{2 \beta^{2}} n\right\rceil\right)\right]+\cos \left[a_{x}\left(\left\lfloor\frac{n}{2}\right\rfloor\right)\right]}{2}+\int_{\left\lceil\frac{\alpha^{2}}{2 \beta^{2}} n\right\rceil}^{\left\lfloor\frac{n}{2}\right\rfloor} \cos \left(a_{x}(t)\right) d t-\int_{\left\lceil\frac{\alpha^{2}}{2 \beta^{2}} n\right\rceil}^{\left\lfloor\frac{n}{2}\right\rfloor} a_{x}^{\prime}(t) \sin \left(a_{x}(t)\right)\left[t-\lfloor t\rfloor-\frac{1}{2}\right] d t .
$$

La deuxième intégrale est contrôlée grossièrement par :

$$
\left|\int_{\left\lceil\frac{\alpha^{2}}{2 \beta^{2}} n\right\rceil}^{\left\lfloor\frac{n}{2}\right\rfloor} a_{x}^{\prime}(t) \sin \left(a_{x}(t)\right)\left[t-\lfloor t\rfloor-\frac{1}{2}\right] d t\right| \leq \frac{4 \arcsin (\beta)}{2}\left(\left\lfloor\frac{n}{2}\right\rfloor-\left\lceil\frac{\alpha^{2}}{2 \beta^{2}} n\right\rceil\right) \leq n \arcsin (\beta)\left(1-\frac{\alpha^{2}}{\beta^{2}}\right) .
$$

Passons au contrôle de la première intégrale. La forme de la minoration (105) nous autorise à supposer $x \neq 0$. Remarquons maintenant que $t \mapsto a_{x}^{\prime}(t)$ est strictement positive et décroissante. En utilisant une intégration par parties, on obtient

$$
\begin{aligned}
\int_{\left\lceil\frac{\alpha^{2}}{\beta^{2}} n\right\rceil}^{\left\lfloor\frac{n}{2}\right\rfloor} \cos \left(a_{x}(t)\right) d t & =\left[\frac{\sin \left(a_{x}(t)\right)}{a_{x}^{\prime}(t)}\right]_{\left\lceil\frac{\alpha^{2}}{2 \beta^{2}} n\right\rceil}^{\left\lfloor\frac{n}{2}\right\rfloor}-\int_{\left\lceil\left\lceil\frac{\alpha^{2}}{2 \beta^{2}} n\right\rceil\right.}^{\left\lfloor\frac{n}{2}\right\rfloor}\left(\frac{1}{a_{x}^{\prime}(t)}\right)^{\prime} \sin \left(a_{x}(t)\right) d t \\
\left|\int_{\left\lceil\frac{\alpha^{2}}{2 \beta^{2}} n\right\rceil}^{\left\lfloor\frac{n}{2}\right\rfloor} \cos \left(a_{x}(t)\right) d t\right| & \leq \frac{1}{a_{x}^{\prime}\left(\left\lfloor\frac{n}{2}\right\rfloor\right)}+\frac{1}{a_{x}^{\prime}\left(\left\lceil\frac{\alpha^{2} n}{2 \beta^{2}}\right\rceil\right)}+\frac{1}{a_{x}^{\prime}\left(\left\lfloor\frac{n}{2}\right\rfloor\right)}-\frac{1}{a_{x}^{\prime}\left(\left\lceil\frac{\alpha^{2} n}{2 \beta^{2}}\right\rceil\right)} \\
& \leq \frac{2}{a_{x}^{\prime}\left(\left\lfloor\frac{n}{2}\right\rfloor\right)} \leq \frac{2}{a_{x}^{\prime}\left(\frac{n}{2}\right)} \leq \frac{\sqrt{2 n+1}}{2|x|} .
\end{aligned}
$$

La définition (115) de $\beta$ nous autorise à majorer

$$
\begin{aligned}
|S(n, x, \alpha, \beta)| & \leq 1+n \arcsin (\beta)\left(1-\frac{\alpha^{2}}{\beta^{2}}\right)+\frac{\sqrt{2 n+1}}{2|x|} \\
& \leq 1+\frac{n}{4}\left(1-\frac{\alpha^{2}}{\beta^{2}}\right)+\frac{2 n}{|x| \sqrt{2 n+1}} .
\end{aligned}
$$

On peut donc choisir $C(2, \alpha)>1$ de sorte que l'inégalité $\frac{C(2, \alpha)}{\sqrt{2 n+1}} \leq|x|$ implique

$$
|S(n, x, \alpha, \beta)| \leq 1+\frac{n}{3}\left(1-\frac{\alpha^{2}}{\beta^{2}}\right) .
$$

On conclut à l'aide de (116).

Deuxième cas : $H(2)$ et $n \in 2 \mathbb{N}+1$. Par invariance radiale et par imparité des fonctions de Hermite $h_{n-2 k}$, on obtient

$$
e_{2, n}(x)=\sum_{k=1}^{\frac{n+1}{2}} h_{2 k-1}(|x|)^{2} h_{n-2 k+1}(0)^{2} \simeq \sum_{k=1}^{\frac{n+1}{2}} h_{2 k-1}(|x|)^{2} \frac{1}{\sqrt{n-2 k+2}} .
$$

En notant $K$ l'ensemble des indices $k$ vérifiant $\frac{\alpha^{2}}{2 \beta^{2}} n+\frac{\alpha^{2}+\beta^{2}}{4 \beta^{2}} \leq k \leq \frac{n+1}{2}$, on a

$$
\begin{aligned}
\forall k \in K \quad \frac{\alpha^{2}}{\beta^{2}}\left(\frac{n}{2}+\frac{1}{4}\right) & \leq k-\frac{1}{4} \\
\alpha \sqrt{2 n+1} & <\beta \sqrt{4 k-1} .
\end{aligned}
$$

En utilisant (114) et la même argumentation que celle du premier cas, nous arrivons à

$$
\begin{aligned}
e_{2, n}(x) & \geq \frac{1}{C(\alpha) \sqrt{n}} \sum_{K} \frac{1}{\sqrt{n-2 k+2}} \sin ^{2}\left[(4 k-1) \Phi\left(\frac{x}{\sqrt{4 k-1}}\right)\right]-\frac{C(\alpha)}{n} \\
& \geq \frac{1}{C(\alpha) n} \sum_{K} \sin ^{2}\left[(4 k-1) \Phi\left(\frac{x}{\sqrt{4 k-1}}\right)\right]-\frac{C(\alpha)}{n} \\
& \gtrsim \alpha \frac{1}{4}\left(1-\frac{\alpha^{2}}{\beta^{2}}\right)-\frac{1}{2 n} \sum_{K} \cos \left[2(4 k-1) \Phi\left(\frac{x}{\sqrt{4 k-1}}\right)\right]-\frac{C(\alpha)}{n} .
\end{aligned}
$$

On finit exactement comme dans le premier cas. 
Troisième cas : $H(d)$ avec $d>2$ et $n \in 2 \mathbb{N}$. On utilise la proposition 4.5 et l'invariance radiale de $e_{d, n}$ exprimée par la formule (111) pour obtenir

$$
\begin{aligned}
e_{d, n}(x) & =\sum_{k=0}^{\frac{n}{2}} e_{d-1,2 k}(\overbrace{|x|, 0, \ldots, 0}^{\in \mathbb{R}^{d-1}}) h_{n-2 k}(0)^{2} \\
& \simeq \sum_{k=0}^{\frac{n}{2}} \frac{e_{d-1,2 k}(|x|, 0, \ldots, 0)}{\sqrt{1+n-2 k}} \\
& \gtrsim \sum_{\frac{\alpha^{2}}{2 \beta^{2}} n \leq k \leq \frac{n}{2}} \frac{e_{d-1,2 k}(|x|, 0, \ldots, 0)}{\sqrt{1+n-2 k}} .
\end{aligned}
$$

Comme dans le premier cas, les indices $k$ sélectionnés vérifient l'inégalité $\alpha \sqrt{2 n+1} \leq \beta \sqrt{2(2 k)+1}$. Puisque l'on a $\beta<\sin \left(\frac{1}{4}\right)$, l'hypothèse de récurrence $H(d-1)$ nous fournit un nombre $C(d-1, \beta)>1$. En imposant la condition $\frac{\beta C(d-1, \beta)}{\alpha \sqrt{2 n+1}} \leq|x| \leq \alpha \sqrt{2 n+1}$, on a

$$
\frac{C(d-1, \beta)}{\sqrt{2(2 k)+1}} \leq|x| \leq \beta \sqrt{2(2 k)+1} .
$$

Cela nous mène à

$$
\begin{aligned}
e_{d, n}(n) & \gtrsim_{d, \beta} \quad \sum_{\substack{\frac{\alpha^{2}}{2 \beta^{2}} n \leq k \leq \frac{n}{2}\\
}} \frac{k^{\frac{d-1}{2}-1}}{\sqrt{1+n-2 k}} \\
& \gtrsim_{d, \beta} n^{\frac{d-1}{2}-1} \sum_{\substack{\frac{\alpha^{2}}{2 \beta^{2}} n \leq k \leq \frac{n}{2} \\
\sqrt{1+n-2 k}}} \frac{1}{\gtrsim_{d, \beta}} n^{\frac{d-1}{2}-1} \times \sqrt{n}=n^{\frac{d}{2}-1} .
\end{aligned}
$$

Dernier cas : $H(d)$ avec $d>2$ et $n \in 2 \mathbb{N}+1$. On se ramène à $H(d-1)$ comme dans le troisième cas.

4.5. Preuve du théorème 4.3, randomisation des fonctions de Hermite. - Nous avons déjà vérifié les hypothèses des théorème 2.6 de dualité et théorème 2.5 d'interpolation (voir (107) et (108)). On a donc les points i) et ii). Décomposant $u=\sum_{n \in \mathbb{N}} \Pi_{n}(u) \in \mathbf{P L}^{p}\left(\mathbb{R}^{d}, \oplus E_{d, n}\right)$, on souhaite maintenant montrer que la norme $\|\cdot\|_{\mathbf{P L}^{p}\left(\mathbb{R}^{d}, \oplus E_{d, n}\right)}$ est équivalente à la suivante

$$
N(u):=\left\|\Pi_{0}(u)\right\|_{L^{2}\left(\mathbb{R}^{d}\right)}+\left[\sum_{n \geq 1} n^{\frac{d}{2}-1}\left(\sum_{k \geq n} \frac{\left\|\Pi_{k}(u)\right\|_{L^{2}\left(\mathbb{R}^{d}\right)}^{2}}{k^{\frac{d}{2}}}\right)^{\frac{p}{2}}\right]^{\frac{1}{p}} .
$$

On va appliquer la proposition 4.1. Quitte à augmenter l'entier $n(d)$, on peut supposer que l'on a $\frac{C(d)}{\sqrt{2 n+1}} \leq$ $1 \leq \alpha \sqrt{2 n+1}$ pour tout $n \geq n(d)$. On vérifie facilement que la norme $\|\cdot\|_{\mathbf{P L}^{p}\left(\mathbb{R}^{d}, \oplus E_{d, n}\right)}$ domine la norme $N$ :

$$
\begin{aligned}
\|u\|_{\mathbf{P L}^{p}\left(\mathbb{R}^{d}, \oplus E_{d, n}\right)}^{p} & \gtrsim d, p \quad \int_{\mathbb{R}^{d}}\left(\sum_{n \geq n(d)}(1+n)^{-(d-1)}\left\|\Pi_{n}(u)\right\|_{L^{2}\left(\mathbb{R}^{d}\right)}^{2} e_{d, n}(x)\right)^{\frac{p}{2}} d x \\
& \gtrsim_{d, p} \int_{\mathbb{R}^{d}}\left(\sum_{n \geq n(d)}(1+n)^{-\frac{d}{2}}\left\|\Pi_{n}(u)\right\|_{L^{2}\left(\mathbb{R}^{d}\right)}^{2} \mathbf{1}_{1 \leq|x| \leq \alpha \sqrt{2 n+1}}\right)^{\frac{p}{2}} d x \\
& \gtrsim_{d, p} \int_{\mathbb{R}^{d}} \sum_{n \geq n(d)}\left(\sum_{k>n}(1+k)^{-\frac{d}{2}}\left\|\Pi_{k}(u)\right\|_{L^{2}\left(\mathbb{R}^{d}\right)}^{2}\right)^{\frac{p}{2}} \mathbf{1}_{\sqrt{2 n+1}<\frac{|x|}{\alpha} \leq \sqrt{2 n+3}} d x \\
& \gtrsim_{d, p} \sum_{n>n(d)} n^{\frac{d}{2}-1}\left(\sum_{k \geq n}(1+k)^{-\frac{d}{2}}\left\|\Pi_{k}(u)\right\|_{L^{2}\left(\mathbb{R}^{d}\right)}^{2}\right)^{\frac{p}{2}} .
\end{aligned}
$$


Pour récupérer les premiers termes d'indice $n \leq n(d)$, il s'agit de remarquer les inégalités triviales

$$
\forall n \in \mathbb{N} \cap[0, n(d)] \quad\|u\|_{\mathbf{P L}^{p}\left(\mathbb{R}^{d}, \oplus E_{d, n}\right)} \gtrsim_{d, p}(1+n)^{-\frac{(d-1)}{2}}\left\|\Pi_{n}(u)\right\|_{L^{2}\left(\mathbb{R}^{d}\right)}\left\|\sqrt{e_{d, n}}\right\|_{L^{p}\left(\mathbb{R}^{d}\right)} .
$$

On obtient alors facilement l'estimation $\|u\|_{\mathbf{P L}^{p}\left(\mathbb{R}^{d}, \oplus E_{d, n}\right)} \gtrsim_{d, p} N(u)$.

Montrons maintenant l'estimation réciproque $\|u\|_{\mathbf{P L}^{p}\left(\mathbb{R}^{d}, \oplus E_{d, n}\right)} \lesssim_{d, p} N(u)$ à l'aide des deux fonctions

$$
\begin{aligned}
A(u, x) & :=\sum_{n \geq 0}(1+n)^{-\frac{d}{2}}\left\|\Pi_{n}(u)\right\|_{L^{2}\left(\mathbb{R}^{d}\right)}^{2} \mathbf{1}_{0 \leq|x| \leq \sqrt{2(2 n+1)}}, \\
B(u, x) & :=e^{-\frac{|x|^{2}}{C(d)}} \sum_{n \geq 0}(1+n)^{-(d-1)}\left\|\Pi_{n}(u)\right\|_{L^{2}\left(\mathbb{R}^{d}\right)}^{2} \mathbf{1}_{\sqrt{2(2 n+1)} \leq|x|},
\end{aligned}
$$

où le terme gaussien provient de la proposition 4.1. D'après la proposition 4.1, nous avons

$$
\|u\|_{\mathbf{P L}^{p}\left(\mathbb{R}^{d}, \oplus E_{d, n}\right)}^{p} \lesssim_{d, p} \int_{\mathbb{R}^{d}}[A(u, x)+B(u, x)]^{\frac{p}{2}} d x \lesssim_{d, p} \int_{\mathbb{R}^{d}} A(u, x)^{\frac{p}{2}}+B(u, x)^{\frac{p}{2}} d x .
$$

Par une argumentation similaire à celle que nous venons d'employer, on vérifie

$$
\int_{\mathbb{R}^{d}} A(u, x)^{\frac{p}{2}} d x \lesssim_{d, p} N(u)^{p} .
$$

Par ailleurs, nous avons trivialement

$$
\begin{aligned}
B(u, x) & \leq e^{-\frac{|x|^{2}}{C(d)}} \sum_{n \geq 0}(1+n)^{-\frac{d}{2}}\left\|\Pi_{n}(u)\right\|_{L^{2}\left(\mathbb{R}^{d}\right)}^{2} \\
& \leq e^{-\frac{|x|^{2}}{C(d)}}\|u\|_{\mathcal{H}^{-\frac{d}{2}}\left(\mathbb{R}^{d}\right)}^{2} \\
\int_{\mathbb{R}^{d}} B(u, x)^{\frac{p}{2}} d x & \lesssim_{d, p} \quad N(u)^{p} .
\end{aligned}
$$

Nous pouvons conclure que l'on a $\|u\|_{\mathbf{P L}^{p}\left(\mathbb{R}^{d}, \oplus E_{d, n}\right)}^{p} \lesssim_{d, p} N(u)^{p}$.

4.6. Preuve du théorème 4.4, injections de Sobolev probabilistes hermitiennes. - Le théorème 4.3 permet par dualité de se ramener au cas $p \in\left[2,+\infty\left[\right.\right.$. Commençons par l'inclusion $\mathcal{H}^{-d\left(\frac{1}{2}-\frac{1}{p}\right)}\left(\mathbb{R}^{d}\right) \subset$ $\mathbf{P L}^{p}\left(\mathbb{R}^{d}, \oplus E_{d, n}\right)$. On invoque l'inégalité triangulaire dans $L^{\frac{p}{2}}\left(\mathbb{R}^{d}\right)$ et $(106)$ pour obtenir pour tout $u \in$ $\mathcal{H}^{-d\left(\frac{1}{2}-\frac{1}{p}\right)}\left(\mathbb{R}^{d}\right)$ :

$$
\begin{aligned}
\left\|\sum_{n \geq 0}\right\| \Pi_{n}(u)\left\|_{L^{2}\left(\mathbb{R}^{d}\right)}^{2} \frac{e_{d, n}}{(1+n)^{d-1}}\right\|_{L^{\frac{p}{2}\left(\mathbb{R}^{d}\right)}} & \leq \sum_{n \geq 0}\left\|\Pi_{n}(u)\right\|_{L^{2}\left(\mathbb{R}^{d}\right)}^{2} \frac{\left\|e_{d, n}\right\|_{L^{\frac{p}{2}}\left(\mathbb{R}^{d}\right)}}{(1+n)^{d-1}} \\
& \leq \sum_{n \geq 0} \frac{\left\|\Pi_{n}(u)\right\|_{L^{2}\left(\mathbb{R}^{d}\right)}^{2}}{(1+n)^{d\left(\frac{1}{2}-\frac{1}{p}\right)}}=\|u\|_{\mathcal{H}^{-d\left(\frac{1}{2}-\frac{1}{p}\right)}}^{2} \mathbb{( \mathbb { R }}^{d)} .
\end{aligned}
$$

Montrons maintenant l'inclusion $\mathbf{P L}^{p}\left(\mathbb{R}^{d}, \oplus E_{d, n}\right) \subset \mathcal{H}^{-d\left(\frac{1}{2}-\frac{1}{p}\right)-\varepsilon}\left(\mathbb{R}^{d}\right)$ pour tout $\varepsilon>0$. Pour tout $u \in$ $\mathbf{P L}^{p}\left(\mathbb{R}^{d}, \oplus E_{d, n}\right)$, on doit montrer

$$
\|u\|_{\mathcal{H}^{-d\left(\frac{1}{2}-\frac{1}{p}\right)^{-\varepsilon}\left(\mathbb{R}^{d}\right)}} \leq C(p, d, \varepsilon)\|u\|_{\mathbf{P L}^{p}\left(\mathbb{R}^{d}, \oplus E_{d, n}\right)} .
$$

Sans perte de généralité, on peut supposer que l'on a $\Pi_{n}(u)=0$ pour $n \gg 1$. Posons à cet effet

$$
\forall n \in \mathbb{N} \quad R_{n}:=\sum_{k \geq n}(1+k)^{-\frac{d}{2}}\left\|\Pi_{k}(u)\right\|_{L^{2}\left(\mathbb{R}^{d}\right)}^{2} .
$$


La décroissance et la positivité de $\left(R_{n}\right)$ permettent d'écrire

$$
\begin{aligned}
\|u\|_{\mathcal{H}^{-d\left(\frac{1}{2}-\frac{1}{p}\right)-\varepsilon}\left(\mathbb{R}^{d}\right)}^{2} & =\sum_{n \in \mathbb{N}}(1+n)^{-d\left(\frac{1}{2}-\frac{1}{p}\right)-\varepsilon}\left\|\Pi_{n}(u)\right\|_{L^{2}\left(\mathbb{R}^{d}\right)}^{2} \\
& =\sum_{n \in \mathbb{N}}(1+n)^{\frac{d}{p}-\varepsilon}\left(R_{n}-R_{n+1}\right) \\
& \leq C(d, p, \varepsilon) \sum_{n \in \mathbb{N}}(1+n)^{\frac{d}{p}-\varepsilon-1} R_{n} \\
& \leq C(d, p, \varepsilon) \sum_{n \in \mathbb{N}} \frac{1}{(1+n)^{\varepsilon+\frac{p-2}{p}} \times(1+n)^{\frac{d-2}{p}} R_{n}} \\
& \leq C(d, p, \varepsilon)\left(\sum_{n \in \mathbb{N}}(1+n)^{\frac{d}{2}-1} R_{n}^{\frac{p}{2}}\right)^{\frac{2}{p}} .
\end{aligned}
$$

On conclut avec (110).

\section{Appendice A}

\section{Optimalité de l'exposant $\max (2, p)$ dans le théorème 2.1}

Il suffit de comprendre le cas unidimensionnel $d_{n}=1$. Examinons l'espace mesuré $X=\mathbb{N} \backslash\{0,1\}$ muni de la mesure de comptage si bien que l'on a $L^{p}(X)=\ell^{p}(\mathbb{N} \backslash\{0,1\})$. Pour tout réel $p \in\left[2, \infty\left[\right.\right.$, on note $\left(X_{n, p}\right)_{n \geq 2}$ une suite i.i.d. de variables aléatoires symétriques, réelles et vérifiant

$$
\forall t \gg 1 \quad \mathbf{P}\left[\left|X_{n, p}\right| \geq t\right]=\frac{\ln (t)}{t^{p}}
$$

Il est clair que $X_{n, p}$ n'a pas de moment d'ordre $p$ et a des moments d'ordre $q \in[1, p[$. On a aussi

$$
\text { p.s. } \quad \sup _{n \geq 2} \frac{\left|X_{n, p}\right|}{n^{\frac{1}{p}} \ln ^{\frac{2}{p}}(n)}=+\infty .
$$

En effet, il s'agit de remarquer que pour tout entier $K \in \mathbb{N}$, on a

$$
\sum_{n \geq 2} \mathbf{P}\left[\left|X_{n, p}\right| \geq K n^{\frac{1}{p}} \ln ^{\frac{2}{p}}(n)\right]=+\infty .
$$

Par indépendance des variables $X_{n, p}$, il existe presque sûrement une infinité d'entiers $n \geq 2$ tels que $\left|X_{n, p}\right| \geq$ $K n^{\frac{1}{p}} \ln ^{\frac{2}{p}}(n)$. On en déduit facilement (118). Revenons à l'optimalité de l'exposant $\max (2, p)$.

Cas $p \in[1,2]$. On fixe $u$ une suite non nulle appartenant à $\ell^{p}(\mathbb{N} \backslash\{0,1\})$ et l'on examine les deux séries aléatoires dans $\ell^{p}(\mathbb{N} \backslash\{0,1\})$

$$
\sum \frac{\varepsilon_{n}}{\sqrt{n} \ln (n)} u \quad \text { et } \quad \sum \frac{X_{n, 2}}{\sqrt{n} \ln (n)} u,
$$

La série aléatoire $\sum \frac{\varepsilon_{n}}{\sqrt{n} \ln (n)}$ converge presque sûrement dans $\mathbb{R}$. Il s'ensuit que la première série aléatoire dans (119) converge presque sûrement dans $\ell^{p}(\mathbb{N} \backslash\{0,1\})$. La divergence presque sûre de la seconde série aléatoire dans (119) découle de (118).

Cas $p \in[2,+\infty[$. Une démarche similaire est valide en examinant les deux séries aléatoires

$$
\sum \frac{\varepsilon_{n}}{n^{\frac{1}{p}} \ln ^{\frac{2}{p}}(n)} w_{n} \quad \text { et } \quad \sum \frac{X_{n, p}}{n^{\frac{1}{p}} \ln ^{\frac{2}{p}}(n)} w_{n},
$$

où $w_{n}=(0, \ldots, 0,1,0, \ldots) \in \ell^{p}(\mathbb{N} \backslash\{0,1\})$ est la suite qui admet 1 à la position $n$ et 0 ailleurs. Il est clair que la première série converge de manière déterministe dans $\ell^{p}(\mathbb{N} \backslash\{0,1\})$. De nouveau, (118) implique la divergence presque sûre de la seconde série aléatoire. 


\section{Appendice B}

Preuve de la proposition 1.10, inégalité de Latała précisée (26)

Notons $B_{n}=\left[\varepsilon_{i j}\right]$ la matrice aléatoire de taille $n \times n$ et dont les coefficients $\varepsilon_{i j}$ sont des variables aléatoires i.i.d. qui suivent une loi $\frac{1}{2}$-Bernoulli à valeurs dans $\{-1,+1\}$. Les inégalités de Kahane-Khintchine (39) dans l'espace de Banach $\left(\mathcal{M}_{n}(\mathbb{R}),\|\cdot\|_{o p}\right)$ nous donnent l'encadrement :

$$
\forall q \in\left[1,+\infty\left[\quad \forall n \in \mathbb{N}^{\star} \quad \mathbf{E}\left[\left\|B_{n}\right\|_{o p}\right] \leq \mathbf{E}\left[\left\|B_{n}\right\|_{o p}^{q}\right]^{\frac{1}{q}} \leq K_{q, 1} \mathbf{E}\left[\left\|B_{n}\right\|_{o p}\right] .\right.\right.
$$

Cela signifie que tous les moments $\mathbf{E}\left[\left\|B_{n}\right\|_{o p}^{q}\right]^{\frac{1}{q}}$ ont le même ordre de grandeur si $n$ tend vers $+\infty$. Par ailleurs, la théorie des matrices aléatoires explique que le moment $\mathbf{E}\left[\left\|B_{n}\right\|_{o p}\right]$ est asymptotiquement de l'ordre de $\sqrt{n}$ (voir [55, Part 2.3]). Nous allons exploiter cette idée pour démontrer l'inégalité (26). Commençons par le lemme élémentaire suivant qui s'apparente à une version commutative de (26).

Lemme B.1. - Considérons un réel $p \in\left[2,+\infty\left[\right.\right.$ ainsi que $N$ variables aléatoires $U_{1}, \ldots, U_{N}$ réelles, centrées, i.i.d. et ayant un moment d'ordre p. Nous avons l'inégalité

$$
\mathbf{E}\left[\left|\frac{U_{1}+\cdots+U_{N}}{\sqrt{N}}\right|^{p}\right] \leq(C \sqrt{p})^{p} \mathbf{E}\left[\left|U_{1}\right|^{p}\right] .
$$

Preuve. L'idée se résume en deux points : on se ramène au cas où les variables $U_{i}$ sont symétriques et l'on invoque les inégalités de Kahane-Khintchine à l'aide du théorème de Fubini. Si nous notons $\widetilde{U}_{1}, \ldots, \widetilde{U}_{N}$ des copies indépendantes des variables $U_{1}, \ldots, U_{N}$ alors l'inégalité de Jensen pour l'espérance en les variables $\widetilde{U}_{1}, \ldots, \widetilde{U}_{N}$ donne

$$
\mathbf{E}\left[\left|U_{1}+\cdots+U_{N}\right|^{p}\right] \leq \mathbf{E}\left[\left|U_{1}-\widetilde{U}_{1}+\cdots+U_{N}-\widetilde{U}_{N}\right|^{p}\right] .
$$

Rappelons alors l'égalité triviale

$$
\mathbf{E}\left[\left|U_{1}-\widetilde{U}_{1}+\cdots+U_{N}-\widetilde{U}_{N}\right|^{p}\right]=\mathbf{E}_{\omega^{\prime}} \mathbf{E}_{\omega}\left[\left|\varepsilon_{1}\left(\omega^{\prime}\right)\left(U_{1}(\omega)-\widetilde{U}_{1}(\omega)\right)+\cdots+\varepsilon_{N}\left(\omega^{\prime}\right)\left(U_{N}(\omega)-\widetilde{U}_{N}(\omega)\right)\right|^{p}\right] .
$$

Il s'agit maintenant d'utiliser le théorème de Fubini, les inégalités de Kahane-Khintchine (39) et (41) (avec $d_{n}=1$ ) pour obtenir

$$
\begin{aligned}
\mathbf{E}\left[\left|U_{1}+\cdots+U_{N}\right|^{p}\right] & \leq \mathbf{E}_{\omega} \mathbf{E}_{\omega^{\prime}}\left[\left|\varepsilon_{1}\left(\omega^{\prime}\right)\right| U_{1}(\omega)-\widetilde{U}_{1}(\omega)\left|+\cdots+\varepsilon_{N}\left(\omega^{\prime}\right)\right| U_{N}(\omega)-\widetilde{U}_{N}(\omega)||^{p}\right] \\
& \leq K_{p, 2}^{p} \mathbf{E}_{\omega}\left[\mathbf{E}_{\omega^{\prime}}\left[\left|\varepsilon_{1}\left(\omega^{\prime}\right)\right| U_{1}(\omega)-\widetilde{U}_{1}(\omega)\left|+\cdots+\varepsilon_{N}\left(\omega^{\prime}\right)\right| U_{N}(\omega)-\left.\widetilde{U}_{N}(\omega)\right|^{2}\right]^{\frac{p}{2}}\right] \\
& \leq(C \sqrt{p})^{p} \mathbf{E}_{\omega}\left[\left(\sum_{i=1}^{N}\left|U_{i}(\omega)-\widetilde{U}_{i}(\omega)\right|^{2}\right)^{\frac{p}{2}}\right] \\
& \leq(C \sqrt{p})^{p}\left(\sum_{i=1}^{N}\left\|\left(U_{i}-\widetilde{U}_{i}\right)^{2}\right\|_{L^{\frac{p}{2}}(\Omega)}\right)^{\frac{p}{2}}=(C \sqrt{p N})^{p}\left\|\left(U_{1}-\widetilde{U}_{1}\right)^{2}\right\|_{L^{\frac{p}{2}(\Omega)}}^{\frac{p}{2}} \\
& \leq(C \sqrt{p N})^{p}\left\|U_{1}\right\|_{L^{p}(\Omega)}^{p} .
\end{aligned}
$$

La preuve précédente est similaire à celle de la proposition 2.10 avec $\left(d_{n}, a_{n}, M_{n}\right)=\left(1,1, U_{n}\right)$ à ceci près que nous pouvons utiliser en plus le principe de symétrisation. Nous avons écrit la preuve précédente d'abord parce que nous aurons besoin plus loin de considérer des variables seulement centrées au lieu de symétriques, mais aussi pour des raisons pédagogiques. En effet, la disparition élémentaire de l'espérance $\mathbf{E}_{\omega^{\prime}}$ de la ligne (120) à la ligne (121) peut être interprétée comme suit : l'espace de Banach $\mathbb{R}$ est de type 2. Nous allons utiliser un substitut non-commutatif de cette propriété pour démontrer (26). C'est l'objet de la proposition suivante dont la preuve est très technique. 
Proposition B.2. - [34, Theorem 1] Il existe une constante universelle $C \geq 1$ telle que pour tout entier $n \in \mathbb{N}^{\star}$, si l'on considère $n^{2}$ variables aléatoires i.i.d. $\left(g_{i j}\right)_{1 \leq i, j \leq n}$ qui suivent une loi normale $\mathcal{N}_{\mathbb{R}}(0,1)$ et une matrice $\left[a_{i j}\right] \in \mathcal{M}_{n}(\mathbb{R})$ alors

$$
\mathbf{E}\left[\left\|a_{i j} g_{i j}\right\|_{o p}\right] \leq C\left(\sqrt[4]{\sum_{i, j=1}^{n} a_{i j}^{4}}+\max _{1 \leq i \leq n} \sqrt{\sum_{j=1}^{n} a_{i j}^{2}}+\max _{1 \leq j \leq n} \sqrt{\sum_{i=1}^{n} a_{i j}^{2}}\right) .
$$

Latała démontre la proposition précédente pour en déduire l'estimation (27) qui se reformule

$$
\mathbf{E}\left[\left\|X_{i j}\right\|_{o p}\right] \leq C \sqrt{n} \mathbf{E}\left[\left|X_{1,1}\right|^{4}\right]^{\frac{1}{4}}
$$

On peut attaquer la preuve de (26) et l'on commence comme dans [34]. On note $\widetilde{X}_{i j}$ des copies indépendantes des variables aléatoires $X_{i j}$. En particulier, on a $\mathbf{E}\left[\widetilde{X}_{i j}\right]=0$. Pour tout réel $p \in[4,+\infty[$, l'argument classique de l'inégalité de Jensen en les variables $\widetilde{X}_{i j}$ donne

$$
\mathbf{E}\left[\left\|X_{i j}\right\|_{o p}^{p}\right] \leq \mathbf{E}\left[\left\|X_{i j}-\tilde{X}_{i j}\right\|_{o p}^{p}\right]=\mathbf{E}\left[\left\|\varepsilon_{i j}\left(X_{i j}-\tilde{X}_{i j}\right)\right\|_{o p}^{p}\right] \leq 2^{p} \mathbf{E}\left[\left\|\varepsilon_{i j} X_{i j}\right\|_{o p}^{p}\right],
$$

où les $n^{2}$ variables aléatoires $\varepsilon_{i j}$ sont indépendantes entre elles et vis-à-vis des variables $X_{i j}$ et $\widetilde{X}_{i j}$. On diffère maintenant de [34] en faisant appel aux inégalités de Kahane-Khintchine (39) dans l'espace de Banach $\left(\mathcal{M}_{n}(\mathbb{R}),\|\cdot\|_{o p}\right)$ afin de récupérer le moment d'ordre $p$ :

$$
\begin{aligned}
\mathbf{E}\left[\left\|\varepsilon_{i j} X_{i j}\right\|_{o p}^{p}\right] & =\mathbf{E}_{\omega} \mathbf{E}_{\omega^{\prime}}\left[\left\|\varepsilon_{i j}\left(\omega^{\prime}\right) X_{i j}(\omega)\right\|_{o p}^{p}\right] \\
& \leq K_{p, 1}^{p} \mathbf{E}_{\omega}\left[\mathbf{E}_{\omega^{\prime}}\left[\left\|\varepsilon_{i j}\left(\omega^{\prime}\right) X_{i j}(\omega)\right\|_{o p}\right]^{p}\right] .
\end{aligned}
$$

Et l'on reprend de nouveau l'argumentation de Latała. En supposant que toutes les variables aléatoires sont indépendantes, (122) et le principe de contraction (voir (45)) donnent pour tout $\omega \in \Omega$

$$
\begin{aligned}
\mathbf{E}_{\omega^{\prime}}\left[\left\|\varepsilon_{i j}\left(\omega^{\prime}\right) X_{i j}(\omega)\right\|_{o p}\right] & \leq \frac{1}{\mathbf{E}\left[\left|g_{11}\right|\right]} \mathbf{E}_{\omega^{\prime}}\left[\left\|g_{i j}\left(\omega^{\prime}\right) X_{i j}(\omega)\right\|_{o p}\right]=\frac{\sqrt{\pi}}{\sqrt{2}} \mathbf{E}_{\omega^{\prime}} \\
& \leq C\left(\sqrt[4]{\sum_{i, j=1}^{n} X_{i j}(\omega)^{4}}+\max _{1 \leq i \leq n} \sqrt{\sum_{j=1}^{n} X_{i j}(\omega)^{2}}+\max _{1 \leq j \leq n} \sqrt{\sum_{i=1}^{n} X_{i j}(\omega)^{2}}\right)
\end{aligned}
$$

Reprenant (123), (124) et tenant compte que les variables $X_{i j}$ sont i.i.d., on obtient

$$
\begin{aligned}
\mathbf{E}\left[\left\|X_{i j}\right\|_{o p}^{p}\right] & \leq C(p) \mathbf{E}\left[\left(\sum_{i, j=1}^{n} X_{i j}^{4}\right)^{\frac{p}{4}}+\max _{1 \leq i \leq n}\left(\sum_{j=1}^{n} X_{i j}^{2}\right)^{\frac{p}{2}}+\max _{1 \leq j \leq n}\left(\sum_{i=1}^{n} X_{i j}^{2}\right)^{\frac{p}{2}}\right] \\
& \leq C(p) \mathbf{E}\left[\left(\sum_{i, j=1}^{n} X_{i j}^{4}\right)^{\frac{p}{4}}+\max _{1 \leq i \leq n}\left(\sum_{j=1}^{n} X_{i j}^{2}\right)^{\frac{p}{2}}\right] .
\end{aligned}
$$

La fin de cette preuve est différente de [34] car on doit tenir compte de l'inégalité $p \geq 4$. Le premier terme ne posera aucun problème tandis que le dernier est plus délicat, c'est pour cela que nous le forçons à être centré en majorant

$$
\sum_{j=1}^{n} X_{i j}^{2} \leq \sum_{j=1}^{n} \mathbf{E}\left[X_{i j}^{2}\right]+\left|\sum_{j=1}^{n} X_{i j}^{2}-\mathbf{E}\left[X_{i j}^{2}\right]\right|
$$


Cela nous donne

$$
\begin{aligned}
\mathbf{E}\left[\left\|X_{i j}\right\|_{o p}^{p}\right] & \leq C(p)[A(1)+A(2)+A(3)], \\
A(1) & :=\mathbf{E}\left[\left(\sum_{i, j=1}^{n} X_{i j}^{4}\right)^{\frac{p}{4}}\right] \\
A(2) & :=\max _{1 \leq i \leq n}\left(\sum_{j=1}^{n} \mathbf{E}\left[X_{i j}^{2}\right]\right)^{\frac{p}{2}}=n^{\frac{p}{2}} \mathbf{E}\left[X_{11}^{2}\right]^{\frac{p}{2}} \\
A(3) & :=\mathbf{E}\left[\max _{1 \leq i \leq n}\left|\sum_{j=1}^{n} X_{i j}^{2}-\mathbf{E}\left[X_{i j}^{2}\right]\right|^{\frac{p}{2}}\right]
\end{aligned}
$$

L'inégalité de Hölder donne d'abord $A(2) \leq n^{\frac{p}{2}} \mathbf{E}\left[\left|X_{11}\right|^{p}\right]$, puis

$$
A(1) \leq \mathbf{E}\left[\left(n^{2}\right)^{\left(1-\frac{4}{p}\right) \frac{p}{4}} \sum_{i, j=1}^{n}\left|X_{i j}\right|^{p}\right]=n^{\frac{p}{2}} \mathbf{E}\left[\left|X_{11}\right|^{p}\right] .
$$

Concernant le terme $A(3)$, nous majorons grossièrement à l'aide du lemme B.1 et de l'inégalité $p \geq 4$

$$
\begin{aligned}
A(3) & \leq \sum_{i=1}^{n} \mathbf{E}\left[\left|\sum_{j=1}^{n} X_{i j}^{2}-\mathbf{E}\left[X_{i j}^{2}\right]\right|^{\frac{p}{2}}\right] \\
& \leq C(p) n^{1+\frac{p}{4}} \mathbf{E}\left[\left|X_{11}^{2}-\mathbf{E}\left[X_{11}^{2}\right]\right|^{\frac{p}{2}}\right] \\
& \leq C(p) n^{1+\frac{p}{4}} \mathbf{E}\left[\left|X_{11}\right|^{p}\right] \\
& \leq C(p) n^{\frac{p}{2}} \mathbf{E}\left[\left|X_{11}\right|^{p}\right] .
\end{aligned}
$$

\section{Appendice $\mathrm{C}$}

Preuve de la proposition 1.10, minoration de la plus petite valeur singulière

On commence par un lemme dual au lemme B.1.

Lemme C.1. - Considérons $N$ variables aléatoires $U_{1}, \ldots, U_{N}$ réelles, centrées, i.i.d. et ayant un moment d'ordre 1. Pour tout $\left(y_{1}, \ldots, y_{N}\right) \in \mathbb{R}^{N}$, nous avons l'inégalité

$$
\mathbf{E}\left[\left|U_{1}\right|\right] \sqrt{y_{1}^{2}+\cdots+y_{N}^{2}} \leq C \mathbf{E}\left[\left|y_{1} U_{1}+\cdots+y_{N} U_{N}\right|\right]
$$

Preuve. Soient $\widetilde{U}_{1}, \ldots, \widetilde{U}_{N}$ des copies indépendantes de $U_{1}, \ldots, U_{N}$. On a

$$
\mathbf{E}\left[\left|y_{1}\left(U_{1}-\widetilde{U}_{1}\right)+\cdots+y_{N}\left(U_{N}-\widetilde{U}_{N}\right)\right|\right] \leq 2 \mathbf{E}\left[\left|y_{1} U_{1}+\cdots+y_{N} U_{N}\right|\right] .
$$

Puisque les variables $U_{i}-\widetilde{U}_{i}$ sont symétriques, le principe de contraction (Théorème 2.16), l'inégalité de Khintchine et l'inégalité de Jensen donnent

$$
\begin{aligned}
\mathbf{E}\left[\left|U_{1}\right|\right] \sqrt{y_{1}^{2}+\cdots+y_{N}^{2}} & =\mathbf{E}\left[\left|U_{1}\right|\right] \mathbf{E}\left[\left|\varepsilon_{1} y_{1}+\cdots+\varepsilon_{N} y_{N}\right|^{2}\right]^{\frac{1}{2}} \\
& \leq \mathbf{E}\left[\left|U_{1}-\widetilde{U}_{1}\right|\right] \times K_{2,1} \mathbf{E}\left[\left|\varepsilon_{1} y_{1}+\cdots+\varepsilon_{N} y_{N}\right|\right] \\
& \leq K_{2,1} \mathbf{E}\left[\left|y_{1}\left(U_{1}-\widetilde{U}_{1}\right)+\cdots+y_{N}\left(U_{N}-\widetilde{U}_{N}\right)\right|\right] \\
& \leq 2 K_{2,1} \mathbf{E}\left[\left|y_{1} U_{1}+\cdots+y_{N} U_{N}\right|\right] .
\end{aligned}
$$


Passons à la preuve de $(25)$. Fixons $y=\left(y_{1}, \ldots, y_{n}\right) \in \mathbb{R}^{n}$. Pour tout $\omega \in \Omega$, la diagonalisation de la matrice symétrique positive $\left|M_{n}(\omega)\right|=\sqrt{{ }^{t} M_{n}(\omega) M_{n}(\omega)}$ dans une base orthonormée fournit l'inégalité

$$
\left|M_{n}(\omega) y\right|^{2}:=\left\langle M_{n}(\omega) y, M_{n}(\omega) y\right\rangle={ }^{t} y\left|M_{n}(\omega)\right|^{2} y \leq\left\|M_{n}(\omega)\right\|_{o p}{ }^{t} y\left|M_{n}(\omega)\right| y .
$$

L'inégalité de Cauchy-Schwarz donne alors

$$
\begin{aligned}
\mathbf{E}_{\omega}\left[\left|M_{n}(\omega) y\right|\right] & \leq \mathbf{E}_{\omega}\left[\sqrt{\left\|M_{n}(\omega)\right\|_{o p}} \sqrt{{ }^{t} y\left|M_{n}(\omega)\right| y}\right] \\
\mathbf{E}_{\omega}\left[\left|M_{n}(\omega) y\right|\right]^{2} & \leq \mathbf{E}_{\omega}\left[\left\|M_{n}(\omega)\right\|_{o p}\right] \mathbf{E}_{\omega}\left[{ }^{t} y\left|M_{n}(\omega)\right| y\right]
\end{aligned}
$$

On invoque alors l'inégalité de Latała (27) pour contrôler le moment d'ordre 1 de $\left\|M_{n}\right\|_{o p}$ :

$$
\mathbf{E}_{\omega}\left[\left|M_{n}(\omega) y\right|\right]^{2} \leq C \mathbf{E}\left[\left|X_{11}\right|^{4}\right]^{\frac{1}{4}} \times{ }^{t} y \mathbf{E}_{\omega}\left[\left|M_{n}(\omega)\right|\right] y
$$

On va maintenant utiliser l'égalité

$$
\left|M_{n}(\omega) y\right|=\sqrt{\frac{1}{n} \sum_{i=1}^{n}\left|\sum_{j=1}^{n} X_{i j}(\omega) y_{j}\right|^{2}}=\left|\left(\frac{1}{\sqrt{n}}\left|\sum_{j=1}^{n} X_{1 j}(\omega) y_{j}\right|, \ldots, \frac{1}{\sqrt{n}}\left|\sum_{j=1}^{n} X_{n j}(\omega) y_{j}\right|\right)\right|
$$

à l'aide de l'inégalité triangulaire entre $\mathbf{E}_{\omega}$ et la norme euclidienne $|\cdot|$ de $\mathbb{R}^{n}$ :

$$
\left(\frac{1}{n} \sum_{i=1}^{n} \mathbf{E}_{\omega}\left[\left|\sum_{j=1}^{n} X_{i j}(\omega) y_{j}\right|\right]^{2}\right)^{1 / 2} \leq \mathbf{E}_{\omega}\left[\left|M_{n}(\omega) y\right|\right] .
$$

Le lemme C.1 nous permet d'obtenir (25) :

$$
\begin{aligned}
\mathbf{E}\left[\left|X_{11}\right|\right]^{2}\left(y_{1}^{2}+\cdots+y_{n}^{2}\right) & \leq C \mathbf{E}_{\omega}\left[\left|M_{n}(\omega) y\right|\right]^{2} \\
\frac{\mathbf{E}\left[\left|X_{11}\right|\right]^{2}}{C \mathbf{E}\left[\left|X_{11}\right|^{4}\right]^{\frac{1}{4}}}\left(y_{1}^{2}+\cdots+y_{n}^{2}\right) & \leq{ }^{t} y \mathbf{E}\left[\left|M_{n}\right|\right] y .
\end{aligned}
$$

Remerciements. L'auteur remercie Didier Robert pour lui avoir signalé l'article [28].

\section{Références}

[1] A. Ayache and N. Tzvetkov. $L^{p}$ properties for Gaussian random series. Trans. Amer. Math. Soc., 360(8):44254439, 2008.

[2] Z.D. Bai, J.W. Silverstein, and Y.Q. Yin. A note on the largest eigenvalue of a large dimensional sample covariance matrix. J. Multivariate Anal., 26(2):166-168, 1988.

[3] J. Bourgain. Periodic nonlinear Schrödinger equation and invariant measures. Comm. Math. Phys., 166(1):1-26, 1994.

[4] J. Bourgain. Invariant measures for the 2D-defocusing nonlinear Schrödinger equation. Comm. Math. Phys., 176:421-445, 1996.

[5] J. Bourgain and A. Bulut. Almost sure global well posedness for the radial nonlinear Schrödinger equation on the unit ball I: the 2D case. Ann. Inst. H. Poincaré Anal. Non Linéaire, 31(6):1267-1288, 2014.

[6] J. Bourgain and A. Bulut. Almost sure global well posedness for the radial nonlinear Schrödinger equation on the unit ball II: the 3D case. J. Eur. Math. Soc, 16:1289-1325, 2014.

[7] J. Bourgain and A. Bulut. Invariant Gibbs measure evolution for the radial nonlinear wave equation on the 3D ball. J. Funct. Anal., 266(4):2319-2340, 2014.

[8] N. Burq, P. Gérard, and N. Tzvetkov. Multilinear eigenfunction estimates and global existence for the three dimensional nonlinear Schrödinger equations. Ann. Scient. Éc. Norm. Supér., 38(2):255-301, 2005.

[9] N. Burq and G. Lebeau. Injections de Sobolev probabilistes et applications. Ann. Sci. Éc. Norm. Supér., 46(6):917-962, 2013.

[10] N. Burq, L. Thomann, and N. Tzvetkov. Long time dynamics for the one dimensional non linear Schrödinger equation. Ann. Inst. Fourier (Grenoble), 63(6):2137-2198, 2013. 
[11] N. Burq, L. Thomann, and N. Tzvetkov. Global infinite energy solutions for the cubic wave equation. Bull. Soc. Math. France, 143(2):301-313, 2015.

[12] N. Burq and N. Tzvetkov. Random data Cauchy theory for supercritical wave equations I: local theory. Invent. Math., 173(3):449-475, 2008.

[13] N. Burq and N. Tzvetkov. Random data Cauchy theory for supercritical wave equations II: a global existence result. Invent. Math., 173(3):477-496, 2008.

[14] N. Burq and N. Tzvetkov. Probabilistic well-posedness for the cubic wave equation. J. Eur. Math. Soc, 16(1):130, 2014.

[15] P. Clement, B. de Pagter, F.A. Sukochev, and H. Witvliet. Schauder decomposition and multiplier theorems. Studia Math., 138(2):135-163, 2000.

[16] J. Creekmore. Type and cotype in Lorentz $L_{p q}$ spaces. Indag. Math., 84(2):145-152, 1981.

[17] A. de Bouard. Construction de solutions pour des edp sur-critiques à données initiales aléatoires. Séminaire Bourbaki, 66(1074), 2013-2014.

[18] A-S. de Suzzoni. Consequences of the choice of a particular basis of $L^{2}\left(S^{3}\right)$ for the cubic wave equation on the sphere and the Euclidean space. Commun. Pure Appl. Anal., 13(3):991-1015, 2014.

[19] Y. Deng. Two-dimensional nonlinear Schrödinger equation with random radial data. Anal. PDE, 5(5):913-960, 2012.

[20] A. Erdélyi. Asymptotic forms for Laguerre polynomials. J. Indian Math. Soc., 24:235-250, 1960.

[21] C. Fefferman. The multiplier problem for the ball. Ann. of Math., 94(2):330-336, 1971.

[22] A. Figà-Talamanca. Radom Fourier Series on Compact Groups. In Theory of Group Representations and Fourier Analysis, pages 1-63. Springer, 1970.

[23] A. Figà-Talamanca and D. Rider. A theorem of Littlewood and lacunary series for compact groups. Pacific J. Math., 16(3):505-514, 1966.

[24] A. Figà-Talamanca and D. Rider. A theorem on random Fourier series on noncommutative groups. Pacific J. Math., 21(3):487-492, 1967.

[25] J. García-Cuerva and J.L. Rubio De Francia. Weighted norm inequalities and related topics. Elsevier, 1985.

[26] L. Grafakos. Classical Fourier analysis, volume 86. Springer, 2008.

[27] S. Grivaux. Almost sure convergence of some random series. C. R. Math. Acad. Sci. Paris, 348:155-159, 2010.

[28] Boris Hanin, Steve Zelditch, and Peng Zhou. Nodal sets of random eigenfunctions for the isotropic harmonic oscillator. Int. Math. Res. Not. IMRN, 2015(13):4813-4839, 2015.

[29] L. Hörmander. The spectral function of an elliptic operator. Acta Math., 121(1):193-218, 1968.

[30] R. Imekraz, D. Robert, and L. Thomann. On random Hermite series. Trans. Amer. Math. Soc., 368(4):27632792, 2016.

[31] N.C. Jain and M.B. Marcus. Integrability of infinite sums of independent vector-valued random variables. Trans. Amer. Math. Soc., 212:1-36, 1975.

[32] J-P. Kahane. Some random series of functions. Heath, (second edition Cambridge 1985), 1968.

[33] H. Koch and D. Tataru. $L^{p}$ eigenfunction bounds for the Hermite operator. Duke Math. J., 128(2):369-392, 2005.

[34] R. Latała. Some estimates of norms of random matrices. Proc. Amer. Math. Soc., 133(5):1273-1282, 2005.

[35] M. Ledoux and M. Talagrand. Probability in Banach Spaces: isoperimetry and processes, volume 23. Springer, 1991.

[36] D. Li and H. Queffélec. Introduction à l'étude des espaces de Banach. Société Mathématique de France, 2004.

[37] J. Lindenstrauss and L. Tzafriri. Classical Banach Spaces: Vol.: 2.: Function Spaces. Springer-Verlag, 1979.

[38] A. Litvak, A. Pajor, M. Rudelson, and N. Tomczak-Jaegermann. Smallest singular value of random matrices and geometry of random polytopes. Adv. Math., 195(2):491-523, 2005.

[39] M.B. Marcus and G. Pisier. Random Fourier Series with Applications to Harmonic Analysis. Annals of Math Studies, Princeton University Press, Princeton, NJ, 101, 1981.

[40] B. Maurey. Type et cotype dans les espaces munis de structures locales inconditionnelles, exposés 24-25. Séminaire Analyse fonctionnelle Ecole Polytechnique, pages 1-25, 1973-74.

[41] B. Maurey and G. Pisier. Séries de variables aléatoires vectorielles indépendantes et propriétés géométriques des espaces de Banach. Studia Math., 58:45-90, 1976.

[42] B. Muckenhoupt. Mean convergence of Hermite and Laguerre series. I. Trans. Amer. Math. Soc., 147(2):419-431, 1970 .

[43] B. Muckenhoupt. Mean convergence of Hermite and Laguerre series. II. Trans. Amer. Math. Soc., 147(2):433460, 1970. 
[44] R.E.A.C. Paley and A. Zygmund. On some series of functions, (1) (2). Proc. Camb. Phil. Soc., 26:337-357, 458-474, 1930.

[45] R.E.A.C. Paley and A. Zygmund. On some series of functions, (3). Proc. Camb. Phil. Soc., 28:190-205, 1932.

[46] A. Poiret, D. Robert, and L. Thomann. Probabilistic global well-posedness for the supercritical nonlinear harmonic oscillator. Anal. PDE, 7(4):997-1026, 2014

[47] A. Poiret, D. Robert, and L. Thomann. Random weighted Sobolev inequalities on $\mathbb{R}^{d}$ and applications to Hermite functions. Ann. Henri Poincaré A, 16(2):651-689, 2015.

[48] D. Robert and L. Thomann. Random weighted Sobolev inequalities and application to quantum ergodicity. Comm. Math. Phys., 335(3):1181-1209, 2015.

[49] M. Rudelson and R. Vershynin. Smallest singular value of a random rectangular matrix. Comm. Pure Appl. Math., 62(12):1707-1739, 2009.

[50] B. Shiffman and S. Zelditch. Random polynomials of high degree and Lévy concentration of measure. Asian J. Math., 7(4):627-646, 2003.

[51] C.D. Sogge. Concerning the $L^{p}$ Norm of Spectral Clusters for Second-Order Elliptic Operators on Compact Manifolds. J. Funct. Anal., 77:123-138, 1988.

[52] C.D. Sogge and S. Zelditch. Lower bounds on the Hausdorff measure of nodal sets. Math. Res. Lett., 18-1:25-37, 2011.

[53] E.M. Stein and G. Weiss. Introduction to harmonic analysis on Euclidean spaces. Princeton, Princeton University Press, 1971.

[54] G. Szego. Orthogonal polynomials. American Mathematical Society, Providence, R.I., fourth edition, 1975. American Mathematical Society, Colloquium Publications, Vol. XXIII.

[55] T. Tao. Topics in random matrix theory, volume 132. American Mathematical Soc., 2012.

[56] S. Thangavelu. Lectures on Hermite and Laguerre expansions., volume 42 of Mathematical Notes. Princeton University Press, Princeton, NJ, 1993.

[57] H. Triebel. Interpolation theory, function spaces, differential operators. North-Holland, 1978.

[58] N. Tzvetkov. Invariant measures for the defocusing nonlinear Schrödinger equation. Ann. Inst. Fourier (Grenoble), 58(7):2543-2604, 2008.

[59] N. Tzvetkov. Riemannian analogue of a Paley-Zygmund theorem. Séminaire EDP, 2008-2009. Exposé no. XV.

[60] L. Weis. Operator-valued Fourier multiplier theorems and maximal $L^{p}$-regularity. Math. Ann., 319(4):735-758, 2001.

[61] K. Yajima and G. Zhang. Local smoothing property and Strichartz inequality for Schrödinger equations with potentials superquadratic at infinity. J. Differential equations, 202(1):81-110, 2004.

[62] Y.Q. Yin, Z.D. Bai, and P.R. Krishnaiah. On the limit of the largest eigenvalue of the large dimensional sample covariance matrix. Probab. Theory Related Fields, 78(4):509-521, 1988.

[63] S. Zelditch. Quantum ergodicity on the sphere. Comm. Math. Phys., 146(1):61-71, 1992.

RAFIK Imekraz, Institut de Mathématiques de Bordeaux, UMR 5251 du CNRS, Université de Bordeaux, 351, cours de la Libération F33405 Talence Cedex, France • E-mail : rafik.imekraz@math.u-bordeaux.fr 